\title{
Classifying and constraining local four photon and four graviton S-matrices
}

\author{
Subham Dutta Chowdhury, ${ }^{a}$ Abhijit Gadde, ${ }^{a}$ Tushar Gopalka, ${ }^{a, c}$ Indranil Halder, ${ }^{a}$ \\ Lavneet Janagal ${ }^{a, b}$ and Shiraz Minwalla ${ }^{a}$ \\ ${ }^{a}$ Department of Theoretical Physics, Tata Institute for Fundamental Research, \\ Mumbai 400005, India \\ ${ }^{b}$ School of Physics, Korea Institute for Advanced Studies, \\ Seoul 02455, Korea \\ ${ }^{c}$ Indian Institute of Science Education and Research, \\ Pune 411008, India \\ E-mail: subham@theory.tifr.res.in, abhijit@theory.tifr.res.in, \\ tushar.gopalka@students.iiserpune.ac.in, \\ indranil.halder@tifr.res.in, lavneet@kias.re.kr, \\ minwalla@theory.tifr.res.in
}

ABstract: We study the space of all kinematically allowed four photon and four graviton S-matrices, polynomial in scattering momenta. We demonstrate that this space is the permutation invariant sector of a module over the ring of polynomials of the Mandelstam invariants $s, t$ and $u$. We construct these modules for every value of the spacetime dimension $D$, and so explicitly count and parameterize the most general four photon and four graviton S-matrix at any given derivative order. We also explicitly list the local Lagrangians that give rise to these S-matrices. We then conjecture that the Regge growth of S-matrices in all physically acceptable classical theories is bounded by $s^{2}$ at fixed $t$. A four parameter subset of the polynomial photon S-matrices constructed above satisfies this Regge criterion. For gravitons, on the other hand, no polynomial addition to the Einstein S-matrix obeys this bound for $D \leq 6$. For $D \geq 7$ there is a single six derivative polynomial Lagrangian consistent with our conjectured Regge growth bound. Our conjecture thus implies that the Einstein four graviton S-matrix does not admit any physically acceptable polynomial modifications for $D \leq 6$. A preliminary analysis also suggests that every finite sum of pole exchange contributions to four graviton scattering also violates our conjectured Regge growth bound, at least when $D \leq 6$, even when the exchanged particles have low spin.

Keywords: Classical Theories of Gravity, Scattering Amplitudes, Conformal Field Theory, Effective Field Theories

ARXIV EPRINT: 1910.14392 


\section{Contents}

1 Introduction $\quad 1$

1.1 Motivation 1

1.2 Three graviton scattering 3

1.3 Classification and parameterization of polynomial four graviton scattering amplitudes 5

1.4 A conjectured bound on Regge scattering 8

$\begin{array}{ll}1.5 \text { Consequences of the CRG conjecture } & 10\end{array}$

$\begin{array}{ll}\text { 1.5.1 Polynomial amplitudes } & 10\end{array}$

$\begin{array}{ll}\text { 1.5.2 Exchange contributions } & 11\end{array}$

$\begin{array}{lll}1.6 & \text { Organization of this paper } & 12\end{array}$

2 Generalities of $2 \rightarrow 2$ S-matrix $r$

$\begin{array}{lll}2.1 & \text { Scattering data } & 13\end{array}$

$\begin{array}{lll}2.1 .1 & \text { Momenta } & 13\end{array}$

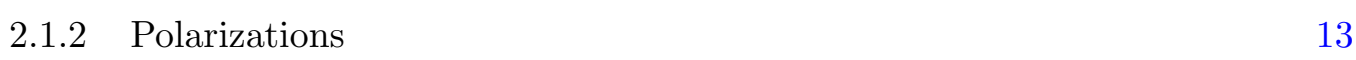

$\begin{array}{lll}2.1 .3 & \text { Unconstrained polarizations } & 14\end{array}$

$\begin{array}{lll}2.2 & S_{4} \text { permutation symmetry } & 17\end{array}$

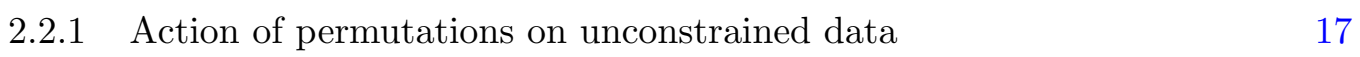

$\begin{array}{ll}2.2 .2 \text { Effective transformations under permutations } & 19\end{array}$

$\begin{array}{lll}2.3 & \text { Permutations: } \mathbb{Z}_{2} \times \mathbb{Z}_{2} \text { and } S_{3} & 19\end{array}$

2.4 Local S-matrices and a module structure 20

$\begin{array}{ll}2.4 .1 & \text { The local module } \\ 2.2 & 20\end{array}$

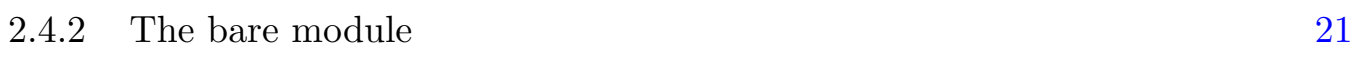

2.4.3 Embedding the local module into the bare module 23

2.5 Irreducible representations of $S_{3}$ and fusion rules 24

2.6 Projecting onto $S_{3}$ singlets 26

2.7 Action of $S_{3}$ on polynomials of $(s, t) \quad 26$

2.8 Standard bases for representations of $S_{3} \quad 28$

2.9 Counting S-matrices 30

2.10 Regge growth 31

3 Generators of the bare module $\quad 34$

3.1 Enumeration 34

3.2 Explicit construction of $e_{I}$ and $S_{3}$ transformations 36

4 Counting local Lagrangians $\quad 40$

4.1 Local Lagrangians and local S-matrices 40

$\begin{array}{lll}4.1 .1 & \text { Scalars } & 41\end{array}$

$\begin{array}{lll}4.1 .2 & \text { Photons } & 42\end{array}$

4.1.3 Gravitons 44 
4.2 Plethystic program for scalars 48

$\begin{array}{lll}4.3 & \text { Photons and gravitons } & 50\end{array}$

4.4 Module generators and Lagrangians 53

5 Polynomial photon S-matrices and corresponding Lagrangians $\quad 54$

5.1 Scalar polynomial S-matrices and corresponding Lagrangians $\quad 55$

5.1.1 Module generators and S-matrix partition functions $\quad 55$

5.1.2 Explicit expressions for most general S-matrix and corresponding Lagrangians $\quad 56$

5.2 Construction of all parity even photon S-matrices for $D \geq 5 \quad 57$

$5.3 D \geq 8 \quad 61$

$\begin{array}{lll}5.4 \quad D=7 & 62\end{array}$

$\begin{array}{lll}5.5 & D=6 & 64\end{array}$

$\begin{array}{lll}5.6 & D=5 & 65\end{array}$

$\begin{array}{lll}5.7 D=4 & 65\end{array}$

$\begin{array}{lll}\text { 5.7.1 Parity even } & 65\end{array}$

$\begin{array}{lll}5.7 .2 & \text { Parity odd } & 67\end{array}$

$\begin{array}{lll}5.8 D=3 & 69\end{array}$

$\begin{array}{lll}\text { 5.8.1 Parity even } & 69\end{array}$

$\begin{array}{lll}\text { 5.8.2 Parity odd } & 71\end{array}$

$\begin{array}{lll}5.9 & \text { Tree level 4-photon S-matrix in string theory } & 72\end{array}$

6 Polynomial graviton S-matrices and corresponding Lagrangians $\quad 73$

$6.1 \quad D \geq 8 \quad 73$

6.1.1 Modules generated by Lagrangians with 8 or fewer derivatives 73

$\begin{array}{ll}\text { 6.1.2 The rest of the local submodule } & 76\end{array}$

$\begin{array}{lll}\text { 6.1.3 Explicit form of S-matrices and Lagrangians } & 78\end{array}$

$\begin{array}{ll}\text { 6.1.4 Comparison with plethystic } & 79\end{array}$

$\begin{array}{ll}6.2 D=7 & 80\end{array}$

$\begin{array}{lll}\text { 6.2.1 Parity odd S-matrices } & 81\end{array}$

$6.3 D=6 \quad 83$

6.3.1 Parity even $\quad 83$

6.3.2 Parity odd $\quad 84$

$\begin{array}{ll}6.4 D=5 & 86\end{array}$

$\begin{array}{llr}6.4 .1 & \text { Parity even } & 86\end{array}$

6.4.2 Parity odd $\quad 86$

$\begin{array}{ll}6.5 D=4 & 87\end{array}$

$\begin{array}{llr}\text { 6.5.1 Parity even } & 87\end{array}$

6.5.2 Parity odd $\quad 88$

$\begin{array}{lll}6.6 & \text { Regge growth of gravity S-matrices } & 89\end{array}$

6.7 Tree level 4-graviton S-matrix in string theory $\quad 89$ 
$\begin{array}{lll}7 & \text { Exchange contributions } & \mathbf{9 0}\end{array}$

$\begin{array}{ll}7.14 \text { graviton scattering from graviton exchange } & 92\end{array}$

$\begin{array}{ll}7.24 \text { graviton scattering from exchanges of massive particles } & 98\end{array}$

$\begin{array}{lll}7.2 .1 & \text { Massive scalar exchange } & 99\end{array}$

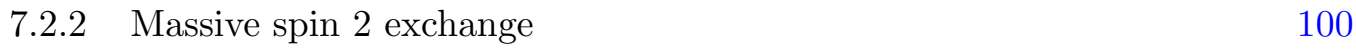

7.3 Exchange contribution to gravitational scattering and Regge growth 104

$\begin{array}{lll}\text { 7.3.1 } & \text { Regge growth of general exchange contributions } & 104\end{array}$

$\begin{array}{lll}\text { 7.3.2 Structure of }\left|\alpha_{i}\right\rangle \text { for the case of gravitational scattering } & 105\end{array}$

$\begin{array}{lll}\text { 7.3.3 } & \text { Counter-term cancellation } & 106\end{array}$

$\begin{array}{ll}\text { 7.3.4 Cancellation between exchange diagrams } & 106\end{array}$

$\begin{array}{lll}8 & \text { Discussion } & 107\end{array}$

$\begin{array}{lr}\text { A Discussion of conjecture 1 } & 109\end{array}$

$\begin{array}{lll}\text { A.1 Universality of the consistent truncation } & 109\end{array}$

$\begin{array}{lll}\text { A.2 Warped compactifications } & 110\end{array}$

$\begin{array}{lll}\text { A.3 Constructing counter examples } & 111\end{array}$

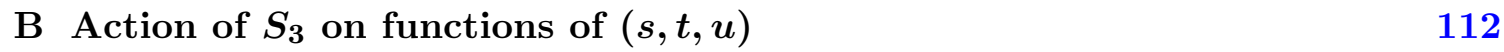

$\begin{array}{lll}\text { B.1 } & S_{3} \text { and its irreducible representations } & 112\end{array}$

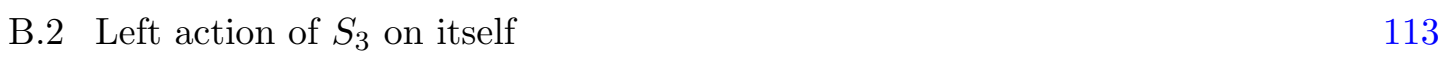

$\begin{array}{lll}\text { B.3 Clebsch-Gordon rules } & 113\end{array}$

$\begin{array}{lll}\text { B.4 Functions of } 3 \text { variables and the permutation group } & 114\end{array}$

$\begin{array}{lll}\text { C } \mathbb{Z}_{2} \times \mathbb{Z}_{2} \text { invariance } & 115\end{array}$

$\begin{array}{lr}\text { D Bare index structures in low dimensions } & 116\end{array}$

$\begin{array}{ll}\text { D.1 Photons } & 117\end{array}$

$\begin{array}{lll}\text { D.2 Gravitons } & 119\end{array}$

$\begin{array}{ll}\text { E Details concerning photon Lagrangians } & 123\end{array}$

$\begin{array}{lll}\text { E.1 Triviality of 3-F structures } & 123\end{array}$

$\begin{array}{lll}\text { E.1.1 } 6 \text { derivatives } & 124\end{array}$

$\begin{array}{ll}\text { E.1.2 } 4 \text { derivatives } & 124\end{array}$

$\begin{array}{lll}\text { E.1.3 } 2 \text { derivatives } & 125\end{array}$

$\begin{array}{lll}\text { E.2 Local photon Lagrangians and polynomial S-matrices } & 125\end{array}$

F S-matrices from Lagrangians no more than cubic in $\boldsymbol{R}_{\alpha \beta \gamma \delta} \quad \mathbf{1 2 6}$

$\begin{array}{lll}\text { F.1 Identities of the Riemann tensor } & 126\end{array}$

$\begin{array}{lll}\text { F.2 Terms quadratic in } R_{\alpha \beta \gamma \delta} & 127\end{array}$

$\begin{array}{lll}\text { F.3 Terms cubic in the Riemann tensor } & 129\end{array}$

$\begin{array}{ll}\text { G Single letter index for photons and gravitons } & 131\end{array}$ 
H.1 $D \geq 10 \quad 133$

$\begin{array}{lll}\text { H.2 } & D<10 & 136\end{array}$

$\begin{array}{ll}\text { I Most general quartic photon Lagrangian } & 137\end{array}$

$\begin{array}{lll}\text { I.1 Terms with } 6 \text { derivatives on } 4 F_{\mu \nu} \text { are all descendants } & 138\end{array}$

$\begin{array}{lll}\text { I.2 Terms with } 4 \text { derivatives on } 4 F_{\mu \nu} \text { are also all descendants } & 138\end{array}$

$\begin{array}{lll}\text { I.3 Primary structures with two derivatives on four field strengths } & 139\end{array}$

J Most general parity even photon S-matrix that grows no faster than $s^{2}$ in the Regge limit

$\begin{array}{ll}\text { K Explicit S-matrices for four graviton scattering } & 143\end{array}$

$\begin{array}{llr}\text { K.1 } & D \geq 8 & 144\end{array}$

$\begin{array}{lll}\text { K.2 } & D=7 & 152\end{array}$

K.3 $D=6 \quad 152$

$\begin{array}{lll}\mathrm{K} .4 & D=5 & 154\end{array}$

$\begin{array}{lll}\text { K.5 } D=4 & 155\end{array}$

$\begin{array}{lll}\text { L Sample exchange contributions to four point scattering } & \mathbf{1 5 7}\end{array}$

$\begin{array}{lll}\text { L.1 Most general exchange contribution to four scalar scattering } & 157\end{array}$

$\begin{array}{ll}\text { L.1.1 Angular dependence and spherical harmonics } & 160\end{array}$

$\begin{array}{lll}\text { L.2 } & \text { Four photon scattering from massive spin } s \text { exchange } & 160\end{array}$

L.3 Massive spin $l$ exchange contribution to 4 graviton scattering 163

\section{Introduction}

\subsection{Motivation}

Consider a compactification of Type II string theory on $R^{p} \times M_{10-p} \cdot{ }^{1}$ The string spectrum on this background includes four dimensional gravitons. Graviton scattering amplitudes at loop level are sensitive probes of the detailed structure of the manifold $M_{10-p}$. At genus zero, however, graviton scattering amplitudes depend on $M_{10-p}$ only through an overall multiplicative factor. When expressed in terms of $G_{p}$, the effective $p$ dimensional Newton constant, these tree amplitudes are completely independent of $M_{10-p}$. These amplitudes are also the same in IIA theory, IIB theory and Type I theory (see appendix A.1 for a discussion).

The universality of tree level graviton scattering amplitudes is a special case of a broader phenomenon. Consider the set of all worldsheet vertex operators that are identity in the $M_{10-p}$ sector and are invariant separately under $(-1)^{F_{L}},(-1)^{F_{R}}$ and $\Omega$ in the $R^{p}$

\footnotetext{
${ }^{1}$ As usual $M_{10-p}$ must be a manifold whose worldsheet sigma model is a $(1,1)$ superconformal field theory with $\widehat{c}=10-p$. For example, when $p=4 M_{6}$ could be a Calabi-Yau manifold.
} 
sector. ${ }^{2}$ Let the collection of spacetime particles corresponding to the BRST cohomology classes of all such vertex operators be denoted by $C_{p}^{\mathrm{II}} \cdot{ }^{3}$ Consider the collection of all tree level S-matrices ${ }^{4}$ with every external particle in $C_{p}^{\mathrm{II}}$. The general result is that these scattering amplitudes are all universal; ${ }^{5}$ moreover every pole in each of these S-matrices results from the exchange of a particle in $C_{p}^{\mathrm{II}}$. There are no poles from the exchange of particles outside the sector $C_{p}^{\mathrm{II}}$. These facts - which follow immediately from the general structure of string worldsheet perturbation theory (see appendix A.1)- have a striking target space interpretation. They imply that the classical target space dynamics of the sub-sector $C^{\mathrm{II}}$ - which we schematically denote by $S\left(C_{p}^{\mathrm{II}}\right)$ - is a universal consistent truncation of classical type II (or type I) string theory on $R^{p} \times M_{10-p}$.

The discussion of the previous paragraphs has an immediate generalization to Heterotic compactifications. Classical graviton scattering amplitudes for the Heterotic string on $R^{p} \times M_{10-p}$ are universal (independent of $M_{10-p}$ ) once they are expressed in terms of $G_{p}$. Once again gravitons in such Heterotic compactifications belong to a collection of particles $C_{p}^{\mathrm{H}}$ Heterotic theories admits a consistent truncation to the universal dynamical system $S\left(C_{p}^{\mathrm{H}}\right) . S\left(C_{p}^{\mathrm{H}}\right)$ is a universal consistent truncation of the classical dynamics of all Heterotic string theories on $R^{p} \times M_{10-p}$.

In the limit $g_{s} \rightarrow 0^{6}$ graviton scattering amplitudes for type II/ Heterotic theory on $R^{p} \times M_{10-p}$ reduce to the tree amplitudes computed using $S\left(C_{p}^{\mathrm{II}}\right)$ or $S\left(C_{p}^{\mathrm{H}}\right)$. Similarly, in the low energy limit string scattering amplitudes in a wide class of compactifications (not necessarily at small $g_{s}$ ) reduce to tree amplitudes computed using the Einstein action

$$
S^{\text {Einstein }}=\int \sqrt{-g} R
$$

We are not aware of any other classical theory which accurately captures string scattering amplitudes in any parametric limit of string theory. ${ }^{7}$

The richness of the now known examples of consistent string compactifications has dampened early hopes that constraints imposed by consistency alone would permit a simple classification of quantum theories of gravity. The discussion above suggests that the situation is much more hopeful in the classical limit. As discussed above, the zoo of known quantum theories of gravity displays a surprising universality in the classical limit. Indeed, to the best of our knowledge, the data from all known string compactifications is consistent with the following bold conjecture ${ }^{8}$

\footnotetext{
${ }^{2} F_{L}$ and $F_{R}$ are the left and right moving worldsheet Fermion number operators while $\Omega$ is the worldsheet orientation reversal operator.

${ }^{3} C_{p}^{\mathrm{II}}$ is, of course, an infinite collection of particles of and includes particles with arbitrary spins.

${ }^{4}$ Expressed in terms of $G_{p}$.

${ }^{5}$ In the sense that they are independent of $M_{10-p}$, and are also the same in IIA theory, IIB theory and Type I theories.

${ }^{6} g_{s}$ is the string coupling.

${ }^{7}$ Classical theories that admit a consistent truncation to one of the three theories - say e.g. $S\left(C_{p}^{\mathrm{II}}\right)-$ yield the same result for gravitational S-matrices as $S\left(C_{p}^{\mathrm{II}}\right)$ itself. For our purposes we thus regard all such theories as equivalent to $S\left(C_{p}^{\mathrm{II}}\right)$.

${ }^{8}$ Conjectures 1-3 were outlined by one of us in a talk at String 2018 [1]. Suggestions very similar to these have also been made over a period of several years by Nima Arkani Hamed (private communication); similar considerations may also have partly motivated the analysis of [2].
} 
- Conjecture 1: there exist exactly three classical gravitational S-matrices ${ }^{9}$ that are consistent with a set of physically motivated 'low energy' constraints (including stability of the vacuum, factorization on poles, causality and positivity of energy). These are the Einstein S-matrix generated by $S^{\text {Einstein }}$, the type II S-matrix generated by $S\left(C_{p}^{\mathrm{II}}\right)$ and the Heterotic S-matrix generated by $S\left(C_{p}^{\mathrm{H}}\right)$.

By the phrase 'classical gravitational S matrices' in the statement above we mean the collection of all S matrices with external particles taken to be any members of minimal classical truncation that includes gravity in the theory under study and not just the $\mathrm{S}$ matrices of gravitons themselves.

Conjecture 1 is very striking but it is also extremely bold, and the evidence in its favour is, as yet, rather limited (see appendix A for a brief discussion). We will not directly study this conjecture - which may well turn out to be incorrect as stated - in this paper. We have nonetheless included a discussion of Conjecture 1 because it implies (but is not implied by) the considerably weaker Conjecture 2 below, which is directly relevant to the analysis of this paper:

- Conjecture 2: the only consistent classical gravitational S-matrix whose exchange poles are bounded in spin is the Einstein S-matrix.

Conjecture 2 in turn implies (but is not implied by) the still weaker conjecture:

- Conjecture 3: the only consistent classical gravitational S-matrix with only graviton exchange poles is the Einstein S-matrix.

We have used observations about string compactifications to motivate this 'Russian doll set' of three successively weaker conjectures. Once motivated, however, these conjectures can be studied on their own terms without any reference to string theory. The direct study, refinement and possible eventual proof of these conjectures appears to us to be a very interesting research program. In this paper we will initiate (or more accurately continue) a study of these conjectures. We will make some progress towards establishing conjectures 3 and 2 . We will not directly study conjecture 1 in this paper; however our technical results may have bearing on related studies in the future.

We emphasize that out considerations apply only to classical S-matrices i.e. S-matrices that do not have non-analyticity apart from a pole corresponding to a tree-level exchange. Loops of gravity as well as matter are parametrically suppressed. In particular, our analysis applies to energy scales parametrically smaller than the Planck scale $M_{p l}$. Potential local corrections to Einstein Lagrangian that we study in this paper are suppressed by a mass scale parametrically smaller than the Planck scale. This is the same regime of applicability as [2].

\section{$1.2 \quad$ Three graviton scattering}

The three conjectures described in the previous subsection apply to $n$ graviton scattering for all $n \geq 3$. The case $n=3$ is particularly simple. It follows from kinematical considerations

\footnotetext{
${ }^{9}$ I.e. S-matrices that are analytic functions of momenta apart from poles.
} 
that the most general 3 graviton S-matrix is a linear combination of the two derivative structure ( see (4.22)), the four derivative structure (see (4.23)) and the six derivative structure (see (4.24)). In other words the most general 3 graviton S-matrix in any theory of gravity - classical or quantum - is specified by three real numbers.

In a classic paper whose results have partly motivated the current work, ${ }^{10}$ Camanho, Edelstein, Maldacena and Zhibeodov (CEMZ) [2] demonstrated that a classical theory with 3 gravitational S-matrices that include a non-zero admixture of the four derivative and six derivative three graviton structures necessarily violates causality unless its four graviton scattering amplitude include contributions from the exchange of poles of arbitrarily high spin. The constraints follow from a particular sign of the Shapiro-time delay which in turn corresponds to the sign of the phase shift in flat space. In AdS similar argument has been made in [3].

Constraints of causality have also been used in the past to constrain sign of certain higher derivative terms in the low energy Lagrangian $[4,5]$.

It thus follows from the results of [2] that the three graviton scattering amplitude is necessarily two derivative - i.e. that of the Einstein theory - in any causal classical theory of gravity whose four graviton S-matrices have exchange contributions that are bounded in spin. In other words CEMZ have already established Conjecture 2 of the previous subsection for the special case of 3 graviton scattering. ${ }^{11}$ The uniqueness of graviton three point function has also been demonstrated using causality in conformal field theory $[6,7]$. In an alternative approach, used in [8], the authors show that if the scattering amplitude obeys a bound in the Regge limit then the effect of shockwaves on a probe commutes which in turn implies the uniqueness of the Einstein gravity three point function.

The CEMZ result already makes a case for the validity of Conjecture 2. We should, however, be careful not to overstate the strength of this evidence. As we have reviewed above, three graviton S-matrix is specified by a finite number of parameters because it is kinematically special. On the other hand four and higher point scattering amplitudes are specified by a finite number of functions of kinematical invariants ( $s$ and $t$ in the case of four graviton scattering) and so an infinite number of real parameters. While the Einstein three graviton scattering amplitude describes a surface of co-dimension 2 in the space of all kinematically allowed 3 graviton scattering amplitudes, the four point (or higher) scattering amplitude describes a surface of infinite codimension in the space of all kinematically allowed classical four (or higher) graviton scattering amplitudes.

It follows that CEMZ type result for four graviton scattering would qualitatively strengthen the evidence for Conjectures 2 or 3 . In the rest of this paper we will focus on the study of four graviton scattering. We will first present an exhaustive kinematical classification and parameterization of local classical four graviton S-matrices and then attempt to cut down the space of allowed S-matrices by proposing a physical criterion that acceptable S-matrices must obey.

\footnotetext{
${ }^{10}$ Conversations with N. Arkani-Hamed, over a period of several years, also form part of the motivation for the current work.

${ }^{11}$ The authors of [2] also rule out exchanges of particles of spin $>2$ when spectrum of such particles is bounded in spin. They do this using the classical Regge growth conjecture stated at the beginning of section 1.4.
} 


\subsection{Classification and parameterization of polynomial four graviton scattering amplitudes}

Consider any classical theory of gravity interacting with other fields. We assume that the equations of motion of our theory are local, i.e. the number of derivatives is finite. When expressed as functions of the polarizations $\epsilon_{i}$ and momenta $p_{i}$ the four graviton S-matrices of any such theory may have poles corresponding to the exchange of particles. Let us focus on the pole corresponding the exchange of the particle $P$. The residue of this pole is completely fixed by the on-shell three particle $g g P$ scattering amplitude.

Now all three particle scattering amplitudes are kinematically fixed to be a linear combination of a finite number - in this case lets say $d_{g}(P)$ - structures. It thus follows that the residue of the four graviton scattering amplitude is a quadratic form in $d_{g}(P)$ undetermined constants (the coefficients behind the $d_{g}(P)$ structures in the three point function). The precise structure of this quadratic form is completely fixed by kinematical considerations. The most general exchange S-matrix is a sum over such a structure for every exchanged particle. Once all pole contributions have been subtracted out, the rest of the amplitude is a polynomial in polarizations and momenta. Below we often refer to this as the 'analytic part' or the 'polynomial part' of the scattering amplitude.

As there is no definite bound on the degree of the polynomial part of the S-matrix, the number of parameters needed to specify the most general polynomial S-matrix is not finite. However polynomial S-matrices can be graded by their dimension (i.e. number of powers of $p_{i}$ ). The number of parameters, $n(m)$, needed to specify the most general dimension $m$ S-matrix is finite. It is convenient to encapsulate the information of $n(m)$ for all $m$ in the S-matrix partition function

$$
Z_{\text {S-matrix }}(x)=\sum_{m=0}^{\infty} n(m) x^{m} .
$$

In the classification part of this paper, among other things, we present explicit results for the partition function (1.1) separately for parity odd and parity even structures, and separately in every dimension. We now briefly explain how we obtain these results, and at the same time accomplish an explicit construction of the space of polynomial S-matrices.

Not every polynomial in the polarizations and momenta is an acceptable S-matrix. Smatrices have to satisfy three additional constraints. First, all acceptable S-matrices must be Lorentz invariant (all indices must contract in pairs or with a Levi-Civita tensor). Second, the S-matrices must obey the constraints of gauge invariance ( see (2.5)). Finally, the S-matrices of four identical Bosonic particles must enjoy invariance under the permutation group $S_{4}$ that permutes the data $\left(\epsilon_{i}, p_{i}\right), i=1 \ldots 4$ of the four scattering particles.

For a reason that will soon become clear, it turns out to be useful to impose the constraint of $S_{4}$ invariance in two steps. $S_{4}$ has a $\mathbb{Z}_{2} \times \mathbb{Z}_{2}$ normal subgroup that leaves the kinematical variables $s, t$, and $u$ (see (2.2)) invariant. The coset space obtained by modding $S_{4}$ out by $\mathbb{Z}_{2} \times \mathbb{Z}_{2}$ is $S_{3}$ (see (2.27)). It follows that we can impose $S_{4}$ invariance by first imposing the constraint of $\mathbb{Z}_{2} \times \mathbb{Z}_{2}$ invariance and later imposing the constraint of $S_{3}$ invariance on the resultant structure. We refer to the set of Lorentz, gauge and 
$\mathbb{Z}_{2} \times \mathbb{Z}_{2}$ invariant polynomials of $\epsilon_{i}$ and $p_{i}$ as 'quasi invariant' S-matrices. As $s, t$ and $u$ are all individually Lorentz, gauge and $\mathbb{Z}_{2} \times \mathbb{Z}_{2}$ invariant, it follows that the product of a quasi invariant S-matrix and any polynomial of $s, t$ and $u$ is itself also a quasi invariant S-matrix. In mathematical parlance the set of gauge and Lorentz invariant polynomials of $p_{i}$ and $\epsilon_{i}$ is a module - which we call the local module (see subsection 2.4) - over the ring of polynomials $s, t$ and $u$. It turns out that the most useful way to think of the space of polynomial S-matrices is to think of it as the $S_{3}$ invariant part of the local module.

The local module is finitely generated and so is completely characterized by its generators, which in turn are labeled by their $S_{3}$ transformations properties and their dimension (i.e. derivative order). In mathematics, the simplest modules are freely generated. It turns out that the local module of quasi invariant S-matrices is freely generated when $D \geq 5$. In this case the partition function (1.1) is completely determined by the spectrum of generators of the module by

$$
Z_{\mathrm{S}-\text { matrix }}(x)=\sum_{J} x^{\Delta_{J}} Z_{\mathbf{R}_{\mathbf{J}}}(x)
$$

where the index $J$ labels the generators of the local module (note, of course, that $J$ has nothing to do with angular momentum), $\Delta_{J}$ is the 'dimension' (more accurately derivative order) of the $J^{t h}$ generator, $\mathbf{R}_{\mathbf{J}}$ is the representation of $S_{3}$ in which the $J^{\text {th }}$ generator transforms and the functions $Z_{\mathbf{R}}(x)$ are listed in (2.44).

The local module is not freely generated when a linear combination of the descendants of different generators vanishes. We refer to all such linear combinations as 'relations' or sometimes as 'null states'. The set of relations themselves form a module. The relation module is completely characterized by its generators. In the context under study in this paper it turns out that the relation modules are themselves always freely generated (i.e. there are no relations for relations). When the local module is not freely generated - this turns out to be the case when $D \leq 4-$ it follows that

$$
Z_{\text {S-matrix }}(x)=\sum_{J} x^{\Delta_{J}} Z_{\mathbf{R}_{J}}(x)-\sum_{I} x^{\Delta_{I}} Z_{\mathbf{R}_{\mathbf{I}}}(x)
$$

where the sum over $J$ runs over all module generators, while the sum over $I$ runs over all relation generators; $\Delta_{I}$ and $\mathbf{R}_{\mathbf{I}}$ are the dimension and $S_{3}$ label of the $I^{\text {th }}$ relation generator.

The reason that the local module is not freely generated in $D=4$ (and $D=3$ ) presumably has to do with the fact that scattering in these dimensions is very special. In particular scattering in $D=4$ is often more conveniently expressed in the spinor helicity formalism than in terms of polarizations as in this paper. Using this formalism a great deal is known about photon and graviton $\mathrm{S}$ matrices in $D=4$ (see e.g. [9] and references therein). It would be interesting to re derive the results of this paper - specialized to $D=4$ - using this formalism. We leave this to future work.

It follows that a complete identification of the generators of the local module (and of the relation module when it exists) immediately determines the partition function over S-matrices (1.1). In sections 5, 6 and related appendices we have explicitly presented all generators of the local module, and also all generators of the relations modules (when they exist). We have also presented an explicit parameterization of all the $S_{3}$ invariant 


\begin{tabular}{|l|l|l|}
\hline dimension & even partition function & odd partition function \\
\hline$D \geq 10$ & $x^{4}\left(2+3 x^{2}+2 x^{4}\right) \mathrm{D}$ & 0 \\
\hline$D=9$ & $x^{4}\left(2+3 x^{2}+2 x^{4}\right) \mathrm{D}$ & 0 \\
\hline$D=8$ & $x^{4}\left(2+3 x^{2}+2 x^{4}\right) \mathrm{D}$ & 0 \\
\hline$D=7$ & $x^{4}\left(2+3 x^{2}+2 x^{4}\right) \mathrm{D}$ & $x^{3} \mathrm{D}$ \\
\hline$D=6$ & $x^{4}\left(2+3 x^{2}+2 x^{4}\right) \mathrm{D}$ & $x^{12} \mathrm{D}$ \\
\hline$D=5$ & $x^{4}\left(2+3 x^{2}+2 x^{4}\right) \mathrm{D}$ & 0 \\
\hline$D=4$ & $x^{4}\left(2+3 x^{2}+2 x^{4}-\left(x^{4}+x^{6}\right)\right) \mathrm{D}$ & $x^{4}\left(1+2 x^{2}+x^{4}-\left(x^{4}+x^{6}\right)\right) \mathrm{D}$ \\
\hline$D=3$ & $x^{4}\left(1+x^{2}+x^{4}-\left(x^{4}+x^{6}\right)\right) \mathrm{D}$ & $x^{9} \mathrm{D}$ \\
\hline
\end{tabular}

Table 1. Final Result for the partition function over 4 photon S-matrices. Here $\mathrm{D}=\frac{1}{\left(1-x^{4}\right)\left(1-x^{6}\right)}$.

\begin{tabular}{|l|l|l|}
\hline dimension & Even partition function & Odd partition function \\
\hline$D \geq 10$ & $x^{8}\left(x^{-2}+6+9 x^{2}+10 x^{4}+3 x^{6}\right) \mathrm{D}$ & 0 \\
\hline$D=9$ & $x^{8}\left(x^{-2}+6+9 x^{2}+10 x^{4}+3 x^{6}\right) \mathrm{D}$ & 0 \\
\hline$D=8$ & $x^{8}\left(x^{-2}+6+9 x^{2}+10 x^{4}+3 x^{6}\right) \mathrm{D}$ & 0 \\
\hline$D=7$ & $x^{8}\left(x^{-2}+6+9 x^{2}+10 x^{4}+3 x^{6}\right) \mathrm{D}$ & $x^{8}\left(2 x^{-1}+3 x+2 x^{3}\right) \mathrm{D}$ \\
\hline$D=6$ & $x^{8}\left(6+9 x^{2}+10 x^{4}+3 x^{6}\right) \mathrm{D}$ & $3 x^{10}\left(x^{2}+x^{4}+x^{6}\right) \mathrm{D}$ \\
\hline$D=5$ & $x^{8}\left(4+7 x^{2}+8 x^{4}+3 x^{6}\right) \mathrm{D}$ & $x^{11}\left(x^{2}+x^{4}+x^{6}\right) \mathrm{D}$ \\
\hline$D=4$ & $x^{8}\left(2+2 x^{2}+3 x^{4}-x^{6}-x^{8}\right) \mathrm{D}$ & $x^{8}\left(1+x^{2}+2 x^{4}-x^{6}-x^{8}\right) \mathrm{D}$ \\
\hline
\end{tabular}

Table 2. Final Result for the partition function over 4 graviton S-matrices.

descendants of these generators, and so an explicit parameterization of all polynomial Smatrices both for the case of 4 photon as well as the case of 4 graviton scattering. The results of these sections permit an immediate computation of the partition function over S-matrices. The final results for the case of photons and gravitons are given in table 1 and 2. The results in this table are presented in terms of the quantity D. ${ }^{12}$

In this paper we have also obtained the results of tables 1 and 2 in a second independent way which we now describe. Every polynomial gauge invariant S-matrix can be obtained from the four graviton (or four photon) part of a finite derivative gauge invariant contact term in Lagrangian. As far as four photon / graviton terms are concerned, almost ${ }^{13}$ all gauge invariant Lagrangians are obtained by taking the products of derivatives of four field strengths / Riemann tensors. Lagrangians that differ off-shell but agree on-shell pro-

\footnotetext{
${ }^{12} \mathrm{D}=\frac{1}{\left(1-x^{4}\right)\left(1-x^{6}\right)}$ is not to be confused with the number of Space-time dimensions $D$.

${ }^{13}$ We describe the exceptions below.
} 
duce the same S-matrix (see section 4 for an explanation of a more careful version of this statement). For this reason the partition function over all polynomial S-matrices can be evaluated (up to a small error that is not difficult to separately account for, see below) by computing the partition function over degree four polynomials of derivatives of the field strength / the Riemann tensor, modulo equations of motion and modulo total derivatives. We have explicitly evaluated these 'Plethystic' partition functions in subsection 4.3 using matrix model techniques. The results of this evaluation are presented in tables 6 and 7 respectively. Similar techniques were used in [10] to compute the partition function on "operator bases" in effective field theories and were generalized to compute partition function on S-matrices in [11]. ${ }^{14}$

We have explained that the plethystic partition function evaluates the S-matrix partition function (1.1) only up to a small error. The error has to do with the fact that it is sometimes possible to find gauge invariant Lagrangians - e.g. Chern Simons Lagrangians - that cannot be constructed out of the product of (derivatives of) four field strengths or four Riemann tensors. Another source of error is that some local Lagrangians generate trivial S-matrices whenever they are total derivatives - even if they are total derivatives of objects (like Chern Simons terms) which themselves cannot be written as products of field strength operators. In sections 5 and 6 we have carefully corrected for each of these errors. Once the corrections are taken into account tables 6 and 7 reduce to tables 1 and 2 presented above, providing a highly non-trivial check of our construction of the most general four graviton and four photon S-matrices.

In sections 5 and 6 and relevant appendices we have also presented an explicit listing of the non linearly gauge invariant Lagrangian whose four photon / four graviton contact term produces each of the polynomial S-matrices that we have explicitly classified and listed in the same sections.

\subsection{A conjectured bound on Regge scattering}

The work reviewed above yields a complete classification and parameterization of all local four graviton S-matrices, analogous to (but much more complicated than) the 3 parameter parameterization of 3 graviton S-matrices. In their classic analysis [2] were able to use physical criteria (the requirement of causality) to constrain the parameters that appeared in the most general three point function. We will now attempt to do the same for the four point function. More specifically, we will constrain classical theories using a conjecture on a bound of the Regge growth of classical scattering amplitudes that we now state

- Classical Regge Growth Conjecture: the S-matrix of a consistent classical theory never grows faster than $s^{2}$ at fixed $t$ - at all physical values of momenta and for every possible choice of the normalized polarization vector $\epsilon_{i}$.

The conditions for 'consistency' of a classical theory in this conjecture are the same as those spelt out in Conjecture 1 earlier in this introduction. In the rest of this subsection

\footnotetext{
${ }^{14}$ We thank T. Hartman for drawing our attention to the papers [10] and [11] while commenting on a preliminary version of this paper.
} 
we will summarize the evidence for the Classical Regge Growth (CRG) Conjecture. In the next subsection we will explore its consequences.

The first piece of evidence in favor of the CRG conjecture is that it is always obeyed by two derivative theories involving particles of spin no greater than two - theories that we independently expect to be consistent. The two derivative nature of interactions ensures that both contact contributions as well as $s$ and $u$ channel exchange contributions grow no faster than $s$ in the Regge limit. $t$ channel exchange graphs on the other hand grow no faster than $s^{J}$ where $J$ is the spin of the exchanged particle. Since we have assumed $J \leq 2$, all contributions obey the CRG conjecture.

The next piece of evidence in support of the conjecture described in this subsection is that it is obeyed by all classical string scattering amplitudes. Recall that, for instance the Type II string scattering amplitudes grow in the Regge limit like

$$
s^{2+\frac{1}{2} \alpha^{\prime} t}
$$

As $t$ is negative at physical values of momenta it follows that this behavior obeys our conjecture. Note also that (1.4) reduces to $s^{2}$ in the limit $\alpha^{\prime} \rightarrow 0$, matching with the fact that gravitational amplitudes, which grow like $\frac{s^{2}}{t}$ in the Regge limit, saturate the CRG bound.

The strongest evidence for the CRG conjecture follows from the observation that the CRG conjecture is tightly related to the chaos bound [12]. We pause to review how this works. Working in a large $N$ unitary CFT in $D \geq 2$ consider a four point $\langle O O O O\rangle$ where $O$ is a real scalar operator and the insertion points are taken to be

$$
\left( \pm \sinh \left(\frac{\tau}{2}\right), \pm \cosh \left(\frac{\tau}{2}\right)\right) \text {. }
$$

All insertions are denoted by the doublet $(t, x)$; insertions all lie completely in the $(t, x)$ plane. The authors of [12] used the unitarity of the CFT to demonstrate that the growth of the connected correlator $\langle O O O O\rangle$ with boost times $\tau$ cannot be faster than $e^{\tau}$. This result holds in the large $N$ limit for boost times $\tau$ large compared to unity but small compared to $\ln N$.

The chaos bound has also been used by Simon Caron-Huot to show that the OPE coefficients are analytic functions of spin and to derive a powerful "inversion formula" for the same [13]. Physical aspects of the inversion formula have been clarified in [14]. The application of the chaos bound to correlators of spinning operators was considered in [15]. ${ }^{15}$

Let us now study a situation in which the CFT under study has a bulk dual. Let the bulk field dual to the operator $O$ be denoted by $\phi$. Let us suppose that the fields $\phi$ in the bulk has a standard quadratic term and also has a have a local four point self interaction of the schematic form $\phi \partial \ldots \partial \phi \partial \ldots \partial \phi \partial \ldots \partial \phi$. One can use the usual rules of AdS/CFT to directly compute the correlator $\langle O O O O\rangle$ of the previous paragraph, and so evaluate its growth with $\tau$. The authors of $[20,21]$ were able to carry through this computation for the most general bulk contact term using the classical bulk theory. They discovered the following interesting fact. Any bulk vertex which, in flat space, would give rise to an S-matrix that grows like $s^{m+1}$ in the Regge limit, turns out to give a contribution to

\footnotetext{
${ }^{15}$ See [16-19] for generalizations.
} 
$\langle O O O O\rangle$ that grows with boost time like $e^{m \tau}$. It follows that at least in AdS space, any bulk interaction associated with a flat-space S-matrix that grows faster than $s^{2}$ at fixed $t$ leads to a boundary correlator that violates a field theory theorem, and so must classically inconsistent. A similar connection between CFT correlators and bulk exchange diagrams has been analyzed in [22].

Although the results described above have been carefully verified only for scalar operator insertions, we feel it is likely that they will continue to hold for insertions of all spins. The tight connection between the CRG and the Chaos bound, is, in our opinion, striking evidence in favor of the CRG conjecture.

Note that the CRG conjecture immediately implies the non existence of a consistent interacting theory of higher spin particles (of bounded spin) propagating in flat space. Let the highest spin in the theory be $J>2$. As the spin $J$ particle is assumed to be interacting, there exists an S-matrix that receives contributions from spin $J$ exchange. In the $t$-channel this exchange contribution scales like $s^{J}$, violating the CRG conjecture. This argument has been used in [2] to rule out the possibility of spectrum of particles of spin $>2$ that is bounded in spin.

\subsection{Consequences of the CRG conjecture}

It is now possible to scan through the explicit results of the classification of S-matrices reviewed in subsection 1.3 and determine the subclass of S-matrices that obeys the CRG conjecture. We first present the complete result of this exercise for polynomial S-matrices and then present some preliminary partial results for exchange amplitudes.

\subsubsection{Polynomial amplitudes}

The scattering of four identical scalars. For $D \geq 3$ the most general local contact scalar interaction term that obeys the CRG bound is given by

$$
S=\int d^{D} x\left(a_{1}\left(\phi^{4}\right)+a_{2}\left(\phi^{2} \partial_{\mu} \partial_{\nu} \phi \partial_{\mu} \partial_{\nu} \phi\right)+a_{3}\left(\phi^{2} \partial_{\mu} \partial_{\nu} \partial_{\rho} \phi \partial_{\mu} \partial_{\nu} \partial_{\rho} \phi\right)\right) .
$$

This implication of the CRG has been used effectively to compute the four point function of certain scalar operators in a theory with slightly broken higher spin symmetry [23].

The scattering of four identical photons. For $D \geq 4$ the most general parity even local contact four photon interaction term that obeys the CRG bound is given by

$$
S=\int d^{D} x\left(a_{1} \operatorname{Tr}\left(\left(F^{2}\right)\right)^{2}+a_{2} \operatorname{Tr}\left(F^{4}\right)+a_{3} \operatorname{Tr}\left(\partial_{\mu} F F \partial^{\mu} F F\right)+a_{4}\left(F_{a b} \operatorname{Tr}\left(\partial_{\alpha} F \partial_{b} F F\right)\right)\right) .
$$

(where matrix multiplication is implied and traces are over Lorentz indices of $F_{\mu \nu}$, and $a_{1}$, $a_{2}, a_{3}$ and $a_{4}$ are constants). In $D=3$ the most general parity even local four photon interaction term that obeys the CRG bound is given by

$$
S=\int d^{3} x\left(a_{2} \operatorname{Tr}\left(F^{4}\right)+a_{3} \operatorname{Tr}\left(\partial_{\mu} F F \partial^{\mu} F F\right)\right) .
$$


Additionally, in special dimensions there are some parity odd local contact Lagrangians that obey the CRG bound. These are the action

$$
S=\int d^{7} x(A \wedge F \wedge F \wedge F)
$$

in $D=7$ and the action

$$
S=\int d^{4} x\left(b_{1} \operatorname{Tr}(F \wedge F) \operatorname{Tr}\left(F^{2}\right)+b_{2} \epsilon_{m n a b} F_{a b} \operatorname{Tr}\left(\partial_{m} F \partial_{n} F F\right)\right) .
$$

in $D=4$, where $b_{1}$ and $b_{2}$ are constants.

The scattering of four identical gravitons. In $D \leq 6$ there are no contact four graviton Lagrangians consistent with the CRG conjecture. For $D \geq 7$ the unique such Lagrangian is the second Lovelock Lagrangian

$$
\chi_{6}=\int \sqrt{-g}\left(\frac{1}{8} \delta_{[a}^{g} \delta_{b}^{h} \delta_{c}^{i} \delta_{d}^{j} \delta_{e}^{k} \delta_{f]}^{l} R_{a b}{ }^{g h} R_{c d}^{i j} R_{e f}{ }^{k l}\right) .
$$

The S-matrix that follows from this Lagrangian is proportional to

$$
\left(\epsilon_{1} \wedge \epsilon_{2} \wedge \epsilon_{3} \wedge \epsilon_{4} \wedge p_{1} \wedge p_{2} \wedge p_{3}\right)^{2}
$$

If we assume the validity of the CRG conjecture, the results just presented amount to a proof of Conjecture 3 for the special case of 4 graviton scattering for $D \leq 6$.

\subsubsection{Exchange contributions}

We have not yet completed a thorough analysis of the Regge behavior of all possible exchange amplitudes; we leave this exercise for future work. In this subsection we summarize a few preliminary results.

Exchange diagrams in the $t$ channel generically scale like $s^{J}$ where $J$ is the largest number of symmetrized indices in the representation that labels the exchanged particle. This $s^{J}$ scaling holds independent of the nature of the external particles, and so applies equally to scalar, photon and graviton scattering. It follows that the exchange of particles with $J \geq 3$ generically violates the CRG conjecture. Exchange of particles with $J \leq 2$ never violates the CRG bound in the $t$ channel but may violate this conjecture in the $s$ and $u$ channels. As the contribution in the $s$ channel and $u$ channel is analytic in $t$, the violations in these channels mean violations at zero impact parameter. This is in contrast with the t-channel where the CRG bound for $J \geq 2$ is violated at finite impact parameter. ${ }^{16}$

The behavior of scattering in the $s$ and $u$ channels is sensitive to the nature of the external scattering particles. In the case of four external scalars or four external photons it is easy to find examples of exchange contributions that do not violate the CRG bound. We have, for example, explicitly computed the contribution to four photon scattering from the exchange of a massive particle of arbitrary mass and demonstrated that its Regge growth is slower than $s^{2}$. In the case of external gravitons, on the other hand, we have shown

\footnotetext{
${ }^{16}$ We thank Douglas Stanford for highlighting this issue to us.
} 
by explicit computation that the exchange of massive scalars or massive spin two particles always leads to an S matrix that violates the CRG bound - and moreover violates it in a manner that cannot be canceled by a compensating local contribution. ${ }^{17}$ The same is also true of exchange of a massless spin two graviton whenever the three graviton scattering amplitude deviates from the Einstein form. More generally we have demonstrated in section 7 - under some hopefully reasonable assumptions - that every exchange contribution to four graviton scattering in $D \leq 6$ - other than graviton exchange with the Einstein three point scattering - violates the CRG bound in a way that cannot be compensated for by local counter term contributions.

Tightened up versions of the arguments described above would amount to a proof of Conjecture 2 for the special case of four graviton scattering for $D \leq 6$. Of course the proof would rely on the validity of the CRG conjecture.

\subsection{Organization of this paper}

In section 2 below we present a discussion of the general structural features of S-matrices for four identical bosons, focusing especially on the module structure of polynomial Smatrices. In section 3 we present an explicit construction of the so called bare module, a freely generated module of 'index structures'. The importance of this construction lies in the fact that the physical local module is a submodule of the bare module. In section 4 we present a discussion of local Lagrangians that give rise to local S-matrices, and present the results of the plethystic counting of local Lagrangians described above. In sections 5 and 6 we present a detailed explicit construction of the local modules of quasi invariant S-matrices for four photon and four graviton scattering. We also present an explicit parameterization of the most general polynomial S-matrices and the contact Lagrangians that give rise to these S-matrices. In section 7 we present a preliminary discussion of the contribution of exchange diagrams to four scalar, four photon and four graviton scattering. We also argue that all such contributions to four graviton scattering appear to violate the CRG bound at least for $D \leq 6$. In section 8 we end with a discussion of open questions and future directions. The details of several calculations and expressions are relegated to appendices.

\section{Generalities of $2 \rightarrow 2$ S-matrix}

In this section we review ${ }^{18}$ and discuss the general structural features of $2 \rightarrow 2$ S-matrices of four identical bosonic scalars, photons or gravitons in an arbitrary number of spacetime dimensions.

\footnotetext{
${ }^{17}$ The AdS version of this statement has previously been argued in [24, 25]. They have shown that in a large $N$ CFT with a spectrum that has a large gap $\Delta_{\text {gap }}$ to higher spin (spin 3 or higher) single trace primaries, the three point function coefficient $\left\langle T_{\mu \nu} T_{\rho \sigma} O\right\rangle$ where $O$ is either a scalar or a spin two operator, is shown to be suppressed by inverse powers of $\Delta_{\text {gap }}$. In [24], the authors use conformal Regge theory and in [25], the authors use average null energy condition to establish the bounds on the three point function coefficients. In the present context, the result should mean that coupling between two gravitons and $\phi$, where $\phi$ is either a scalar or a spin two particle, must vanish when there are no exchange poles corresponding to higher spin particles.

${ }^{18}$ In particular $2.12 .3,2.5$ contain only well known results, and have been included in this paper only to establish notation and to jog the reader's memory.
} 


\section{$2.1 \quad$ Scattering data}

\subsubsection{Momenta}

Consider the scattering of four massless particles in $D$-dimensional Minkowski space. Let $p_{i}^{\mu}$ be momentum of the $i^{\text {th }}$ particle. The masslessness of the scattering particles and momentum conservation means

$$
p_{i}^{2}=0, \quad \sum_{i=1}^{4} p_{i}^{\mu}=0 .
$$

We use the mostly positive convention and define Mandelstam variables,

$$
\begin{aligned}
s & :=-\left(p_{1}+p_{2}\right)^{2}=-\left(p_{3}+p_{4}\right)^{2}=-2 p_{1} \cdot p_{2}=-2 p_{3} \cdot p_{4} \\
t & :=-\left(p_{1}+p_{3}\right)^{2}=-\left(p_{2}+p_{4}\right)^{2}=-2 p_{1} \cdot p_{3}=-2 p_{2} \cdot p_{4} \\
u & :=-\left(p_{1}+p_{4}\right)^{2}=-\left(p_{2}+p_{3}\right)^{2}=-2 p_{1} \cdot p_{4}=-2 p_{2} \cdot p_{3} .
\end{aligned}
$$

The equalities in (2.2) follow from (2.1). Thanks to momentum conservation $s+t+u=0$. In the rest of the paper when we need to make a specific choice of independent Mandelstam variables we will usually take these to $s$ and $t .{ }^{19}$

\subsubsection{Polarizations}

The $2 \rightarrow 2$ S-matrix is a Lorentz invariant complex valued function of the momenta $p_{i}$, together with the data that specifies the internal or spin degree of freedom of each scattering particle.

Scalars. Scalar particles have no internal degrees of freedom, so $2 \rightarrow 2$ scalar S-matrices are functions only of momenta. For $D \geq 4$ Lorentz invariance ensures that S-matrices are, in fact, functions only of $s$ and $t$. In the special case $D=3$, four scalar S-matrices can be either parity even or parity odd. Parity even S-matrices are simply a function of $s$ and $t$ as in higher dimensions. Parity odd S-matrices are given by $\epsilon_{\mu \nu \rho} p_{1}^{\mu} p_{2}^{\nu} p_{3}^{\rho}$ times a second function of $s$ and $t$.

Photons. The internal degree of freedom of a photon may be taken to be its polarization vector $\epsilon_{i}^{\mu}$. In the Lorentz gauge (which we use throughout this paper in order to preserve manifest Lorentz invariance),

$$
\epsilon_{i} \cdot p_{i}=0
$$

The S-matrix must also be invariant under residual gauge transformations i.e. under the transformations,

$$
\epsilon_{i}^{\mu} \rightarrow \epsilon_{i}^{\mu}+\zeta\left(p_{i}\right) p_{i}^{\mu}
$$

We will sometimes use the notation

$$
\zeta\left(p_{i}\right)=\zeta_{i} .
$$

\footnotetext{
${ }^{19}$ In the special case $D=2$ the kinematics of four particle scattering degenerates and variables $u$ and $t$ can be solved for in terms of $s$. Through this paper we will, however, always assume that $D \geq 3$, and so $s$ and $t$ are always independent variables.
} 
As $\zeta\left(p_{i}\right)$ is a completely arbitrary function of $p_{i}{ }^{20}$ the four numbers $\zeta_{i}$ can be varied independently of each other. It follows that the requirement of gauge invariance is simply the condition that the S-matrix is separately invariant under each of the transformations

$$
\epsilon_{i}^{\mu} \rightarrow \epsilon_{i}^{\mu}+\zeta_{i} p_{i}^{\mu}
$$

separately for each $i$.

To summarize, a 4-photon S-matrix is a Lorentz invariant complex valued function $\mathcal{S}\left(p_{i}, \epsilon_{i}\right)$, subject to the condition (2.3). It depends linearly on each of the four polarizations $\epsilon_{i}^{\mu}$ and is separately invariant under each of the four shifts (2.5).

Gravitons. The internal degrees of freedom of a graviton can be parameterized by its traceless symmetric polarization tensor $h_{i}^{\mu \nu}$. In Lorentz gauge,

$$
h_{i}^{\mu \nu} p_{i}^{\nu}=0
$$

As before, the S-matrix enjoys invariance under residual gauge transformations,

$$
h_{i}^{\mu \nu} \rightarrow h_{i}^{\mu \nu}+\zeta_{i}^{\mu} p_{i}^{\nu}+\zeta_{i}^{\nu} p_{i}^{\mu}, \quad \text { where } \quad \zeta_{i} \cdot p_{i}=0 .
$$

Through most of this paper we will find it convenient to specialize to the special choice of polarization

$$
h_{\mu \nu}^{i}=\epsilon_{\mu}^{i} \epsilon_{\nu}^{i} \quad \text { where } \quad k_{i} \cdot \epsilon_{i}=0, \quad \epsilon_{i} \cdot \epsilon_{i}=0
$$

The gauge transformation parameter $\zeta_{i}^{\mu}=\zeta_{i} \epsilon_{\mu}^{i}$, preserves the choice of the polarization (2.8) and induces the gauge transformations

$$
\epsilon_{i}^{\mu} \rightarrow \epsilon_{i}^{\mu}+\zeta_{i} p_{i}^{\mu}
$$

These transformations are same as the ones in $(2.4) .{ }^{21}$

In conclusion, with the choice 2.8, a 4 -graviton S-matrix $\mathcal{S}\left(p_{i}, \epsilon_{i}\right)$, like the photon, is a Lorentz invariant complex valued function $\mathcal{S}\left(p_{i}, \epsilon_{i}\right)$ but this time one that is a bilinear function of each of the $\epsilon_{i}{ }^{\prime}$ 's, subjected to the tracelessness condition $\epsilon_{i} \cdot \epsilon_{i}=0$.

\subsubsection{Unconstrained polarizations}

In the previous subsubsection we have expressed the S-matrix as shift invariant functions of the polarization vectors $\epsilon_{i}$. It is possible to simultaneously 'solve' for the constraints

\footnotetext{
${ }^{20}$ And as two of the particles participating in a four particle scattering event never have identical $D$ momenta.

${ }^{21}$ The special choice (2.8) does not result in loss of generality. Let $S(\epsilon)$ denote the S-matrix with a single special choice of polarization, $h_{1}^{\mu \nu}=\epsilon^{\mu} \epsilon^{\nu}$. Then the linear combination

$$
S(u+v)-S(u)-S(v)
$$

where $u$ and $v$ are orthogonal polarization vectors, yields the S-matrix for the choice of polarization $h_{1}^{\mu \nu}=$ $u^{\mu} v^{\nu}+v^{\mu} u^{\nu}$ and this sort of polarizations form a basis for general symmetric traceless tensors $h_{1}^{\mu \nu}$. As the S-matrix is linear in $h_{1}^{\mu \nu}$, the S-matrix with the choice (2.8) carries the same information as the most general 4-graviton S-matrix.
} 
on $\epsilon_{i}$ (tracelessness) and the constraints on the S-matrix (shift or gauge invariance) and re express the S-matrix as a function of independent unconstrained variables as follows.

The momenta $p_{i}$ span a three dimensional subspace of $D$-dimensional Minkowski space. We refer to this subspace as the scattering 3-plane. The polarization vectors $\epsilon_{i}$ can be decomposed into part transverse to the scattering plane $\epsilon_{i}^{\perp}$ and part parallel to the scattering plane $\epsilon_{i}^{\|}$

$$
\epsilon_{i}=\epsilon_{i}^{\perp}+\epsilon_{i}^{\|}
$$

The condition $\epsilon_{i} \cdot p_{i}=\epsilon_{i}^{\|} \cdot p_{i}=0$ forces $\epsilon_{i}^{\|}$to lie in a two dimensional subspace of the scattering plane. Moreover the constraint that S-matrices are invariant under the shifts $\epsilon_{i}^{\|} \rightarrow \epsilon_{i}^{\|}+p_{i}$ tells us that the S-matrix is a function only of one of the two free components of $\epsilon_{i}^{\|}$. It follows that - for the purpose of evaluating gauge invariant S-matrices - the set of inequivalent vectors $\epsilon_{i}^{\|}$may be parameterized by a single complex number $\alpha_{i}$. We choose the following (arbitrary) parameterization that obeys $\epsilon_{i}^{\|} \cdot p_{i}=0$.

$$
\begin{aligned}
\epsilon_{1}^{\|} & =\alpha_{1} \sqrt{\frac{s t}{u}}\left(\frac{p_{2}}{s}-\frac{p_{3}}{t}\right)+a_{1} p_{1} \\
\epsilon_{2}^{\|} & =\alpha_{2} \sqrt{\frac{s t}{u}}\left(\frac{p_{1}}{s}-\frac{p_{4}}{t}\right)+a_{2} p_{2} \\
\epsilon_{3}^{\|} & =\alpha_{3} \sqrt{\frac{s t}{u}}\left(\frac{p_{4}}{s}-\frac{p_{1}}{t}\right)+a_{3} p_{3} \\
\epsilon_{4}^{\|} & =\alpha_{4} \sqrt{\frac{s t}{u}}\left(\frac{p_{3}}{s}-\frac{p_{2}}{t}\right)+a_{4} p_{4} .
\end{aligned}
$$

The numbers $a_{i}$ represent the freedom to shift $\epsilon_{i}$ by gauge transformations; $a_{i}$ are redundancies of description and will not show up in any gauge invariant physical result. On the other hand the parameters $\alpha_{i}$ are physical. In particular

$$
\epsilon_{i}^{\|} \cdot\left(\epsilon_{i}^{\|}\right)^{*}=\left|\alpha_{i}\right|^{2}
$$

(see also (2.16) below).

With these definitions in place we can write

$$
\epsilon_{i}=\epsilon_{i}^{\perp}+\epsilon_{i}^{\|} .
$$

Equations (2.13) and (2.11) express the $D$ component vector $\epsilon_{i}$ in terms of the $D-3$ component vector $\epsilon_{i}^{\perp}$ and the single parameter $\alpha_{i}$ and the redundant variables $a_{i}$.

Unlike $\epsilon_{i}$, the pair $\left(\epsilon_{i}^{\perp}, \alpha_{i}\right)$ are unconstrained data in the case of photons. In the case of gravitons the data still has to obey the single constraint ${ }^{22}$ - which is a consequence of the tracelessness of $\epsilon_{i}$

$$
\epsilon_{i}^{\perp} \cdot \epsilon_{i}^{\perp}+\alpha_{i}^{2}=0
$$

\footnotetext{
${ }^{22}$ It is sometimes also useful to view the polarizations $\epsilon_{i}$ as normalized according to the condition

$$
\epsilon_{i} \cdot \epsilon_{i}^{*}=1 \quad \Rightarrow \quad\left|\epsilon_{i}^{\perp}\right|^{2}+\left|\alpha_{i}\right|^{2}=1 .
$$

Notice that $\epsilon_{i}$ and $\epsilon_{i}+p_{i}$ have the same norm, so this condition is gauge invariant. We will not need to impose this normalization condition in this paper.
} 
This constraint can be used to solve for $\epsilon_{i}^{\perp} . \epsilon_{i}^{\perp}$ in terms of $\alpha_{i}^{2}$. In enumerating contraction structures we simply omit all terms containing factors of $\epsilon_{i}^{\perp} \cdot \epsilon_{i}^{\perp}$. For counting purposes, therefore, $\epsilon_{i}^{\perp}$ can effectively be treated as null.

The expressions (2.11) and (2.13) allow us to convert any Lorentz and gauge invariant parity even expression for a photon/graviton S-matrix, initially presented as a function of $\epsilon_{i}$ and $p_{i}$, into an function of $\left(\epsilon_{i}^{\perp}, \alpha_{i}\right)$ and $(s, t)$. This function is separately linear/bilinear in $\left(\epsilon_{i}^{\perp}, \alpha_{i}\right)$. Note that the individual momenta $p_{i}$ enter into this reduced form of the S-matrix only through ${ }^{23}(s, t)$.

The converse of the assertion of the previous paragraph also holds. The translation formulae $e^{24}$

$$
\begin{aligned}
& \alpha_{1}=2 \frac{p_{2} \cdot F^{1} \cdot p_{3}}{\sqrt{s t u}}, \quad \alpha_{2}=2 \frac{p_{1} \cdot F^{2} \cdot p_{4}}{\sqrt{s t u}}, \quad \alpha_{3}=2 \frac{p_{4} \cdot F^{3} \cdot p_{1}}{\sqrt{s t u}}, \quad \alpha_{4}=2 \frac{p_{3} \cdot F^{4} \cdot p_{2}}{\sqrt{s t u}} . \\
& \epsilon_{1}^{\perp}=-2 \frac{p_{2} \cdot F^{1}}{s}+2 \frac{p_{2} \cdot F^{1} \cdot p_{3}}{t u} p_{3}-2 \frac{p_{2} \cdot F^{1} \cdot p_{3}}{t s} p_{1}-2 \frac{p_{2} \cdot F^{1} \cdot p_{3}}{s u} p_{2} \\
& \epsilon_{2}^{\perp}=-2 \frac{p_{1} \cdot F^{2}}{s}+2 \frac{p_{1} \cdot F^{2} \cdot p_{3}}{t u} p_{3}-2 \frac{p_{1} F^{2} \cdot p_{3}}{u s} p_{2}-2 \frac{p_{1} \cdot F^{2} \cdot p_{3}}{t s} p_{1} \\
& \epsilon_{3}^{\perp}=-2 \frac{p_{2} \cdot F^{3}}{u}+2 \frac{p_{2} \cdot F^{3} \cdot p_{1}}{t s} p_{1}-2 \frac{p_{2} \cdot F^{3} \cdot p_{1}}{t u} p_{3}-2 \frac{p_{2} \cdot F^{3} \cdot p_{1}}{s u} p_{2} \\
& \epsilon_{4}^{\perp}=-2 \frac{p_{2} \cdot F^{4}}{t}+2 \frac{p_{2} \cdot F^{4} \cdot p_{3}}{s u} p_{3}-2 \frac{p_{2} \cdot F^{4} \cdot p_{3}}{t s} p_{4}-2 \frac{p_{2} \cdot F^{4} \cdot p_{3}}{t u} p_{2} .
\end{aligned}
$$

where

$$
F_{\mu \nu}^{i}=p_{\mu}^{i} \epsilon_{\nu}^{i}-p_{\nu}^{i} \epsilon_{\mu}^{i}
$$

is the manifestly gauge invariant Field strength and $a . F . b \equiv a^{\mu} F_{\mu \nu} b^{\nu}$, allow us to re-express any $\mathrm{SO}(D-3)$ parity invariant function of $\left(\epsilon_{i}^{\perp}, \alpha_{i}\right)$ and $(s, t)$ with the correct homogeneity properties as a manifestly Lorentz and gauge invariant S-matrix.

There is a slight subtlety in the discussion of parity odd S-matrices, i.e. S-matrices constructed out of a single factor of the $D$-dimensional Levi-Civita tensor $\varepsilon$. The reason these structures are subtle is simply that $\varepsilon$ tensors in different numbers of dimensions have different numbers of indices and so do not simply map to one another. In order to resolve this subtlety it will prove convenient to formally regard the $\varepsilon$ tensor as one of the arguments of parity odd $S$-matrices. From this viewpoint, a parity odd S-matrix is a Lorentz and gauge invariant function of $p_{i}, \epsilon_{i}$ and $\varepsilon$ that has the property that is linear in $\varepsilon$ (it also has the usual homogeneities in $\epsilon_{i}$ ). We now define a $D-3$ dimensional $\widetilde{\varepsilon}^{D-3}$ tensor by the equation,

$$
\widetilde{\varepsilon}^{D-3}=\varepsilon_{\mu_{1} \ldots \mu_{D-3} \mu_{D-2} \mu_{D-1} \mu_{D}} p_{1}^{\mu_{D-2}} p_{2}^{\mu_{D-1}} p_{3}^{\mu_{D}} .
$$

\footnotetext{
${ }^{23}$ This follows from the fact $\epsilon_{i}^{\perp} \cdot p_{j}=0$. While $\epsilon_{i}^{\|} \cdot p_{j} \neq 0$ the result of this dot product is given by $\alpha_{i}$ times an easily computed function of $(s, t)$.

${ }^{24}$ In (2.17) we have presented one of many inequivalent looking — but actually equal — gauge and Lorentz invariant expressions for $\alpha_{i}$ and $\epsilon_{i}^{\perp}$. For instance the second of (2.17) follows from the observation that the expression $-2 \frac{p_{2} \cdot F^{1}}{s}$ equals $\epsilon_{1}^{\perp}$ plus a vector in the scattering three plane. $\epsilon_{1}^{\perp}$ is then isolated by removing the unwanted vector via the subtractions in the last three terms on the r.h.s. of the second line of (2.17). It is also true, however, that $-2 \frac{p_{3} \cdot F^{1}}{t}$ is proportional to $\epsilon_{1}^{\perp}$ plus a vector in the scattering 3 plane. This observation leads to a different looking — but actually equal — expression of the schematic form $\epsilon_{1}^{\perp}=-2 \frac{p_{3} \cdot F^{1}}{t}+$ subtractions.
} 
Note that $\widetilde{\varepsilon}^{D-3}$ is totally anti-symmetric under the $S_{4}$ permutation of particles. It has momentum degree three. Note that $\widetilde{\varepsilon}^{D-3}$ is proportional to $(s t u)^{\frac{1}{2}} \varepsilon^{D-3}$, where $\varepsilon^{D-3}$ is a $D-3$ dimensional Levi-Civita tensor. For this reason it is sometimes useful to work with the 'normalized' tensor

$$
N\left(\widetilde{\varepsilon}^{D-3}\right)=\frac{\widetilde{\varepsilon}_{\mu_{1} \ldots \mu_{D-3}}}{\sqrt{s t u}}
$$

$N\left(\widetilde{\varepsilon}^{D-3}\right)$ is proportional to the Levi-Civita tensor in $D-3$ dimensions up to a sign. ${ }^{25}$ The action of the permutation group on $\widetilde{\varepsilon}^{D-3}$ and $N\left(\widetilde{\varepsilon}^{D-3}\right)$ is given by

$$
P\left(\widetilde{\varepsilon}^{D-3}\right)=(-1)^{\operatorname{sgn}(P)} \widetilde{\varepsilon}^{D-3}, \quad P\left(N\left(\widetilde{\varepsilon}^{D-3}\right)\right)=(-1)^{\operatorname{sgn}(P)} N\left(\widetilde{\varepsilon}^{D-3}\right)
$$

where $P$ is an arbitrary permutation in $S_{4}$ of the momenta $p_{1} \ldots p_{4}$. In other words both $\widetilde{\varepsilon}^{D-3}$ and $N\left(\widetilde{\varepsilon}^{D-3}\right)$ pick up a sign under every odd permutation (e.g. under single exchange permutations).

When $D$ is odd, we will choose to regard every parity odd S-matrix a function of $\widetilde{\varepsilon}^{D-3}, \epsilon_{i}^{\perp}, \alpha_{i}$ and $(s, t)$; the function in question is linear in $\widetilde{\varepsilon}^{D-3}$ (it also has the usual homogeneities in $\left.\left(\epsilon_{i}^{\perp}, \alpha_{i}\right)\right)$. When $D$ is even, on the other hand, we will choose to regard every parity odd S-matrix a function of $N\left(\widetilde{\varepsilon}^{D-3}\right), \epsilon_{i}^{\perp}, \alpha_{i}$ and $(s, t)$; once again the function is linear in $N\left(\widetilde{\varepsilon}^{D-3}\right)$ and has the usual homogeneities in $\left(\epsilon_{i}^{\perp}, \alpha_{i}\right) \cdot{ }^{26}$

For parity odd structures, the formulae (2.17), (2.16), supplemented with (2.19) and (2.20), map any $\mathrm{SO}(D-3)$ invariant function of $\left(\epsilon_{i}^{\perp}, \alpha_{i}\right),(s, t)$ and $\widetilde{\varepsilon}^{D-3}$ (or $N\left(\widetilde{\varepsilon}^{D-3}\right)$ ) that is linear in $\widetilde{\varepsilon}^{D-3}$ - and with the appropriate homogeneity properties in $\left(\epsilon_{i}^{\perp}, \alpha_{i}\right)$ to a manifestly Lorentz and gauge invariant S-matrix that is linear in $\varepsilon$ and therefore is parity odd.

\section{$2.2 \quad S_{4}$ permutation symmetry}

Apart from the requirements of gauge and Lorentz invariance, the $2 \rightarrow 2$ S-matrix for identical bosonic particles also enjoys invariance under $S_{4}$, the group that permutes the four scattering particles. We now turn to a brief analysis of the action of this Bose symmetry and its consequences.

\subsubsection{Action of permutations on unconstrained data}

In this subsection we discuss the action of the permutation group $S_{4}$ act on the scattering data $\left(p_{i}, \epsilon_{i}^{\perp}, \alpha_{i}\right)$. It turns out that this action is not as trivial as one might first guess.

\footnotetext{
${ }^{25}$ Note that the l.h.s. of (2.20) is precisely defined (unlike the $D-3$ Levi-Civita tensor which is precisely defined only once we specify an orientation in the $D-3$ plane orthogonal to the scattering plane).

${ }^{26}$ The reason we make this distinction between odd and even $D$ will become clearer later in this paper. Roughly speaking the reason goes as follows. We will see below that S-matrices can be expanded in a sort of Taylor Series in momenta. In every dimension the basis functions of this expansion for parity even Smatrices all have even powers of momenta. As far as parity odd S-matrices go, however, the basis functions are even in momenta when $D$ is even; the fact that $N\left(\widetilde{\varepsilon}^{D-3}\right)$ is also even in powers of momenta makes (2.20) a natural building block of such S-matrices. On the other hand the building blocks for parity odd S-matrices in odd $D$ are odd in momenta; the fact that (2.19) is cubic in momenta makes it a natural building block for S-matrices in this case.
} 
Let $B=\left(p_{1}, p_{2}, p_{3}, p_{4}\right), C=\left(\alpha_{1}, \alpha_{2}, \alpha_{3}, \alpha_{4}\right)$ and $D=\left(\epsilon_{1}^{\perp}, \epsilon_{2}^{\perp}, \epsilon_{3}^{\perp}, \epsilon_{4}^{\perp}\right)$ each represent column vectors of length 4 . Let $P$ be any permutation in $S_{4}$. Let $M(P)$ denote the representation of $S_{4}$ in its 'defining' (reducible) 4 dimensional representation. Clearly the column vectors $B$ and $D$ transform under permutations as

$$
B \rightarrow M(P) B, \quad D \rightarrow M(P) D
$$

(2.22) asserts that $P$ acts on $p_{i}$ and $\epsilon_{i}^{\perp}$ by permuting the $i$ indices; this follows from definitions.

On the other hand permutations act on $C$ in a more complicated way. This is because the quantities $\alpha_{i}$ are not completely physical; the equations (2.16) that define $\alpha_{i}$ in terms of gauge invariant data express $\alpha_{i}$ as $F_{\mu \nu}^{i}$ with indices contracted with two of the other (non $i$ ) scattering momenta. Which momenta we choose to contract $F_{\mu \nu}^{i}$ changes the answer only up to a sign. As there is no permutation invariant way of making a choice of contracted momenta, the action of permutations on $\alpha_{i}$ permutes $\alpha_{i}$ but only up to a sign. ${ }^{27}$ It follows that on general grounds the action of permutations on $C$ must take the form

$$
C \rightarrow D(P) M(P) C
$$

where $D(P)$ is a diagonal matrix with diagonal entries \pm 1 . In other words $C$ transforms in a projective representation of $S_{4}$ with the phase ambiguities given by \pm 1 . A simple explicit computation using (2.16) demonstrates that $D(P)=(-1)^{\operatorname{sgn}(\mathrm{P})}$ so that the action of permutations on $C$ is given by

$$
C \rightarrow(-1)^{\operatorname{sgn}(\mathrm{P})} M(P) C .
$$

Note that the 'anomaly' in the transformation properties of $\alpha_{i}$ is extremely simple. With our choice of basis it is a phase that acts in the same way on all four $\alpha_{i}$. It follows immediately that the action of permutations on even polynomials of $\alpha_{i}$ is 'non-anomalous' whereas the action of permutations on odd polynomials of $\alpha_{i}$ is the naive action times $(-1)^{\operatorname{sgn}(\mathrm{P})}$.

Parity even S-matrices are even functions of $\alpha_{i}$ in every dimension. ${ }^{28}$ On the other hand parity odd S-matrices are even function of the $\epsilon_{i}^{\perp}$ (and hence of $\alpha_{i}$ ) when $D$ is odd, but an odd function of both $\epsilon_{i}^{\perp}$ and $\alpha_{i}$ when $D$ is even. ${ }^{29}$

We have already explained (see around (2.21) ) that it is convenient to regard parity odd S-matrices as functions of $\widetilde{\varepsilon}^{D-3}$ (see (2.19)) when $D$ is odd, but of $N\left(\widetilde{\varepsilon}^{D-3}\right)$ (see (2.20))

\footnotetext{
${ }^{27}$ We can also see this in another way. It follows from (2.12) that $\left|\alpha_{i}\right|^{2}$ is completely physical, so permutations act on $\left|\alpha_{i}\right|^{2}$ in the usual way; once again we see that the ambiguity is in the sign.

${ }^{28}$ The overall S-matrix is of combined degree 4 in $\alpha, \epsilon^{\perp}$ variables (in the case of photon scattering) and of combined degree 8 in $\alpha$ and $\epsilon^{\perp}$ (for the case of graviton scattering). Because the $\epsilon_{i}^{\perp}$ always contract in pairs in even scattering amplitudes, it follows that every term in such a scattering amplitude is an even function of $\alpha_{i}$.

${ }^{29}$ This follows from the observation that in parity odd S-matrices exactly $D-3$ of indices of the $\varepsilon$ tensor have to be contracted with the $\epsilon_{i}^{\perp}$; other $\epsilon_{j}^{\perp}$ s then contract in pairs. Thus the number of $\epsilon_{i}^{\perp}-$ hence $\alpha_{i}-$ that appear in any given term in a parity odd S-matrix is odd if $D-3$ is odd but even if $D-3$ is even.
} 
when $D$ is even. We have also seen that $\widetilde{\varepsilon}^{D-3}$ and $N\left(\widetilde{\varepsilon}^{D-3}\right)$ both transform under permutations as (2.21). As far as S-matrices go, it is thus possible to absorb the anomalous phase in the transformation of $\alpha_{i}$ (see (2.23)) into a renormalized permutation transformation rule for $N\left(\widetilde{\varepsilon}^{D-3}\right)$ as we summarize in detail in the next subsubsection.

\subsubsection{Effective transformations under permutations}

It follows from the analysis of the previous subsubsection that, as far as S-matrices go the correct permutation transformation laws are captured by the following effective rules. $p_{i}$, $\alpha_{i}$ and $\epsilon_{i}^{\perp}$ all transform under permutations in the simple 'non-anomalous' manner

$$
B \rightarrow M(P) B, \quad D \rightarrow M(P) D, \quad C \rightarrow M(P) C
$$

Parity even S-matrices are separately of homogeneity one (two) in $\alpha_{i}, \epsilon_{i}^{\perp}$ for photon (graviton) S-matrices. Parity odd S-matrices also have the same homogeneity in $\alpha_{i}, \epsilon_{i}^{\perp}$, but in addition are linear in $\widetilde{\varepsilon}^{D-3}$ (see (2.19)) when $D$ is odd and in $N\left(\widetilde{\varepsilon}^{D-3}\right)$ when $D$ is even. $\widetilde{\varepsilon}^{D-3}$ transforms under permutations as

$$
P\left(\widetilde{\varepsilon}^{D-3}\right)=(-1)^{\operatorname{sgn}(P)} \widetilde{\varepsilon}^{D-3},
$$

while $N\left(\widetilde{\varepsilon}^{D-3}\right)$ are assigned the transformation properties

$$
P\left(N\left(\widetilde{\varepsilon}^{D-3}\right)\right)=N\left(\widetilde{\varepsilon}^{D-3}\right)
$$

i.e. is invariant under permutations. We will use the rules summarized in this subsubsection to compute the transformations of S-matrices under permutations in the rest of this paper.

\subsection{Permutations: $\mathbb{Z}_{2} \times \mathbb{Z}_{2}$ and $S_{3}$}

The permutation group $S_{4}$ has a special abelian subgroup $\mathbb{Z}_{2} \times \mathbb{Z}_{2}$ generated by (2143), (3412) i.e. the subgroup of double transpositions. ${ }^{30}$ The importance of this subgroup is that it leaves all the Mandelstam variables $s, t$ and $u$ invariant.

Another feature of this subgroup is that it is normal. ${ }^{31}$ As a result, the $\operatorname{coset}^{32} S_{4} /\left(\mathbb{Z}_{2} \times\right.$ $\mathbb{Z}_{2}$ ) inherits the group structure of $S_{4}$. The coset group is easily identified. Every $S_{4}$ group element is $\mathbb{Z}_{2} \times \mathbb{Z}_{2}$ equivalent to a unique element of the form ( $\left.a b c 4\right)$. It follows that the $\mathbb{Z}_{2} \times \mathbb{Z}_{2}$ 'gauge invariance' can be fixed by adopting the 'gauge fixing condition' that particle 4 is not permuted. This choice of gauge fixing clearly reveals the coset to be simply the $S_{3}$ that permutes particles 1,2 and 3. Thus we conclude that

$$
\frac{S_{4}}{\left(\mathbb{Z}_{2} \times \mathbb{Z}_{2}\right)}=S_{3}
$$

\footnotetext{
${ }^{30}$ We label an element of $S_{4}$ by the image of (1234) under that element. The group $\mathbb{Z}_{2} \times \mathbb{Z}_{2}$ consists of the two listed generators together with the identity permutation (1234) and a fourth element (4321).

${ }^{31}$ Recall that a subgroup $H \subset G$ is normal if it obeys the property that for any $h \in H, g h g^{-1} \in H$ for all $g \in G$. In other words, the normal subgroup is fixed by the adjoint action of the group.

${ }^{32}$ Here the coset is either by left action or by right action, both cosets are equivalent because subgroup is normal.
} 
It follows that the condition of $S_{4}$ invariance on the S-matrices can be imposed in two steps. In the first step, we impose only $\mathbb{Z}_{2} \times \mathbb{Z}_{2}$ symmetry on the gauge invariant functions of $\epsilon_{i}^{\mu}$ and $p_{i}^{\mu}$ (with the necessary homogeneity properties in $\epsilon_{i}^{\mu}$ ). We call the S-matrices thus obtained, quasi-invariant S-matrices. The coset group $S_{3}$ acts linearly on the space of quasi-invariant S-matrices. In order to obtain fully $S_{4}$ invariant S-matrices we must further project the space of quasi-invariant S-matrices down to its $S_{3}$ invariant subspace. The importance of the notion of quasi-invariant S-matrices lies in the fact that the multiplication of a quasi-invariant S-matrix by a function of $s, t$ leaves it quasi-invariant because $(s, t)$ are themselves individually invariant under $\mathbb{Z}_{2} \times \mathbb{Z}_{2}$. It follows that the space of not-necessarily-polynomial quasi-invariant S-matrices forms a vector space over functions of $(s, t)$. It is intuitively clear - and we will see in great detail below - that this vector space is finite dimensional.

\subsection{Local S-matrices and a module structure}

This paper is principally devoted to a study of a special class of S-matrices which we call local S-matrices. We define these objects as S-matrices that are polynomial functions of $\epsilon_{i}$ and $p_{i}$. In this subsection we turn our attention to such S-matrices. We focus, first on quasi-invariant S-matrices, postponing the task of enforcing $S_{3}$ invariance to later.

\subsubsection{The local module}

It is clear that the set of local S-matrices is closed under multiplication by any polynomial $p(s, t)$ and addition. This structure is reminiscent of the vector space except for one important difference. Polynomials of $(s, t)$ do not form a field but rather only a ring i.e. they do not have multiplicative inverse. ${ }^{33}$ Consequently, the set of local quasi-invariant S-matrices forms a module, over the ring of polynomials of $(s, t)$ and not a vector space. The identification of space of local S-matrices with a module over a ring of polynomials of Mandelstam variables has also been made in [11].

Viewed as a vector space over the field of complex numbers, the space of local quasiinvariant S-matrices is, of course, infinite dimensional. Viewed as a module, however, this space is 'finitely generated' as we now explain. We pause to introduce some (standard) mathematical terminology.

The elements of the form $r \cdot m$, where $m$ is a given element of the module and $r$ is any element of the ring, are said to form the span of $m$. We call the elements in the span of $m$, the descendants of $m \cdot{ }^{34}$ Sometimes we denote the descendant of $m$ in a more physical notation $r|m\rangle$. A subset $G=\left\{g_{i}\right\}$ of the module $M$ is said to generate $M$ if the

\footnotetext{
${ }^{33}$ This is a very important difference. In a genuine vector space if a vector $a$ is a multiple of a vector $b$ then it is also true that the vector $b$ is a multiple of the vector $a$. In a module, on the other hand, if $a$ equals a ring element times $b$ then it is usually not true that $b$ equals a ring element times $a$. In other words the notion of proportionality is inherently hierarchical in a ring. We elaborate on this below.

${ }^{34}$ This is non-standard mathematical terminology but being physicists we connect well to the word "descendant". Note that the set of basis vectors of a conformal multiplet can be thought of as a module generated by the primary operator over the ring of polynomials in $P_{\mu}$. From this point of view, conformal descendants are descendants in our sense. Similarly, the Verma module can be thought of as the module generated by the primary operator over the ring of Virasoro creation operators.
} 
smallest submodule which contains $G$ is $M$ itself. In other words, the union of spans of all descendants of $g_{i}$ is $M$ itself. A module $M$ is said to be finitely generated if it has a finite generator (i.e. a generator with a finite number of elements). A generator set $G$ is said to generate $M$ freely if the following condition holds,

$$
\sum_{i} r_{i} \cdot g_{i}=0 \quad \text { iff } \quad \text { all } r_{i}=0 .
$$

In other words, every element of $m$ is a unique linear combination of $g_{i}$ over the ring. A module $M$ is a free module if there exists a $G$ that generates it freely. In this case the generator set $G$ is called the basis of $M$. A free module is the next best thing after a vector space. Understanding its structure is equivalent to understanding its basis elements. When the module is not free, one has to characterize the module by giving its generators and their relations. ${ }^{35}$

We can find the generators of the module of local quasi-invariant S-matrices in following way. These S-matrices are obtained from local Lagrangians. We first look for a basis over complex numbers of local quasi-invariant S-matrices of the lowest degree. Next we again look for a basis over complex numbers of local quasi-invariant S-matrices of lowest degree that are not in the span of the previously chosen elements, and so on. This process terminates at a finite degree - intuitively because the gauge invariant field strengths built out of $\epsilon_{i}$ have a finite number of indices ${ }^{36}-$ more on this later. It follows that the module of local quasi-invariant S-matrices is finitely generated. We will call this module, the local module for short and will label its generators as $E_{J}\left(p_{i}, \epsilon_{i}\right)$ and the generator set as $L$.

As the local S-matrices are constrained by transversality and shift invariance of polarizations $\epsilon_{i}$, and as these constraints involve the momenta $p_{i}$ that in turn define $s, t$ and $u$, it is much less clear that this module is freely generated, and, indeed we will find below that this is not always the case.

\subsubsection{The bare module}

As we have already explained in subsubsection 2.1.3, the equations (2.11) and (2.13) allow us to re-express any photon/graviton quasi-invariant S-matrix as a polynomial of $\left(\epsilon_{i}^{\perp}, \alpha_{i}\right)$ that is simultaneously of degree one/two in each of these pairs of variables; the coefficients of this polynomial expressions are functions of $(s, t)$.

We now turn to a crucial point. If we start with a local quasi-invariant S-matrix, it is possible to show that the resulting expression, written as a polynomial of $\left(\epsilon_{i}^{\perp}, \alpha_{i}\right)$, using (2.11) and (2.13), has coefficients that are polynomials (rather than generic functions) of $(s, t) .{ }^{37}$ We will discuss this observation momentarily in the next subsubsection.

\footnotetext{
${ }^{35}$ If the relations do not form a free module, then one has to characterize the relation module in the same way and so on. This is called the free resolution of a module.

${ }^{36}$ Once all these indices are contracted with momenta, the remaining momentum indices have to contract with each other yielding powers of $s, t$ and hence belonging to the span of lower degree structures.

${ }^{37}$ This statement is not obvious as the r.h.s. of (2.11) involves expressions that are not polynomial in $s, t$ and $u$. And indeed individual Lorentz invariant building blocks of S-matrices - like $p_{2} . F^{1} . p_{3}$ (see (2.16)) are not individually polynomials in $p$ and $\epsilon$. When we put these building blocks together, however, we always recover polynomials. For instance the product of four terms $-p_{2} \cdot F^{1} \cdot p_{3}$ and a similar term for particle 2,3 and 4 - is proportional to $(s t u)^{2} \alpha_{1} \alpha_{2} \alpha_{3} \alpha_{4}$ (see (2.16)). We have checked by explicit computation in many different contexts, that this is the case. See, e.g., (5.19), (5.52), (5.70) for explicit examples worked out in detail.
} 
This motivates us to define a new module. We define the parity even part of the module of bare quasi-invariant photon/graviton S-matrices, or bare module for short, over the ring of polynomials of $(s, t)$, to be the set of parity even (i.e. $\widetilde{\varepsilon}^{D-3}$ independent ) rotationally invariant and $\mathbb{Z}_{2} \times \mathbb{Z}_{2}$ invariant polynomials of $\left(\epsilon_{i}^{\perp}, \alpha_{i}\right)$ and $(s, t)$ that are simultaneously of degree one/two in each of the pair of variables $\left(\epsilon_{i}^{\perp}, \alpha_{i}\right) .{ }^{38}$ Any basis of the vector space - over the field of complex numbers - of rotationally invariant polynomials of $\left(\epsilon_{i}^{\perp}, \alpha_{i}\right)$ (subjected to the requirement of $\mathbb{Z}_{2} \times \mathbb{Z}_{2}$ invariance and appropriate homogeneity requirements) forms a generating set for this module. ${ }^{39}$ Let us denote this generator set as $B$ and its elements as $e_{I}\left(\alpha_{i}, \epsilon_{i}^{\perp}\right)$. We sometimes call $e_{I}$ "index structures". Notice that our generators are all independent of $s, t$ and so, in particular, are of zero homogeneity in derivatives. As the variables $\epsilon_{i}^{\perp}$ and $\alpha_{i}$ are completely unconstrained (in the case of photons) or obey only the momentum independent constraint (2.43) (in the case of gravitons), it is clear that this choice generates our module finitely and freely. This makes $B$ the basis of the bare module. The key point - made at the beginning of this subsubsection - is that the local module is a submodule of the free bare module.

The parity odd part of the bare module is defined in odd/even $D$ in a similar manner, to be set of rotationally invariant and $\mathbb{Z}_{2} \times \mathbb{Z}_{2}$ invariant polynomials of $\left(\epsilon_{i}^{\perp}, \alpha_{i}\right),(s, t)$ and $\widetilde{\varepsilon}^{D-3}$ (resp. $\left.N\left(\widetilde{\varepsilon}^{D-3}\right)\right)$ that are linear in $\widetilde{\varepsilon}^{D-3}$ (resp. $\left.N\left(\widetilde{\varepsilon}^{D-3}\right)\right)$ and are also simultaneously of degree one or two (corresponding to photons and gravitons) in each of the pair of variables $\left(\epsilon_{i}^{\perp}, \alpha_{i}\right)$. Basis elements are functions of $\widetilde{\varepsilon}^{D-3}$, (resp. $\left.N\left(\widetilde{\varepsilon}^{D-3}\right)\right) \alpha_{i}, \epsilon_{i}^{\perp}$ only; there is no further dependence on $s$ and $t$. Note that these basis elements are of dimension zero in even $D$ but of dimension 3 in odd $D$.

Like its even component, the parity odd part of the bare module is also free. Once again the module of parity odd local S-matrices is a submodule of the parity odd part of the bare module.

These observations allows us to carry out the program of characterizing the local module in two steps. In the first step - carried out in detail in section 3, we first completely characterize the bare module by identifying $e_{I}$ 's. This step is relatively simple. In the second step (which we briefly discuss in the next subsubsection and then implement in detail in the subsequent sections of this paper) we understand the embedding of the local module into the free bare module, and thereby understand its structure and enumerate its elements.

\footnotetext{
${ }^{38}$ In the case of the gravitational S-matrix, the variables $\left(\epsilon_{i}^{\perp}, \alpha_{i}\right)$ are also constrained to obey (2.15).

${ }^{39}$ At the end of subsection (2.3) we had explained that the set of not necessarily local quasi-invariant S-matrices constitute a vector space over the field of functions of $s, t, u$. The generators of the bare module clearly also define a basis for this vector space.
} 


\subsubsection{Embedding the local module into the bare module}

Remarkably we find that the generators of the local module $E_{J}\left(p_{i}, \epsilon_{i}\right) \in L$ are related to the basis elements $e_{I}\left(\alpha_{i}, \epsilon_{i}^{\perp}\right) \in B$ of the bare module as,

$$
E_{J}\left(p_{i}, \epsilon_{i}\right)=\sum_{e_{I} \in B} p_{I J}(s, t) e_{I}\left(\alpha_{i}, \epsilon_{i}^{\perp}\right) .
$$

where $p_{I J}(s, t)$ are polynomials ${ }^{40}$ of $s, t$.

In our study of photon/graviton S-matrices later in this paper we encounter two cases. In the first case, (this holds for both photon and graviton scattering when $D \geq 5$ ), $|L|=$ $|B|{ }^{41}$ In the second case, which turns out to apply to both photon and graviton scattering in $D=4$ and also photon scattering in $D=3,|L|>|B|$. In this subsection we briefly discuss these two cases in turn.

Let us first consider the case $|L|=|B|$. In this case the local module is freely generated if and only if the equation

$$
\sum_{E_{J} \in L} r^{J}(s, t) E_{J}\left(p_{i}, \epsilon_{i}\right)=0
$$

has no non-trivial solutions for polynomials $r^{J}(s, t)$. Plugging the expansion (2.29) into (2.30) and equating coefficients of $e_{I}$ we find that (2.30) turns into

$$
\sum_{J} p_{I J}(s, t) r^{J}(s, t)=0
$$

For each value of $s$ and $t,(2.31)$ is a set of $|B|$ linear equations for $|B|$ variables. This set of equations has non-trivial solutions if and only if

$$
\operatorname{Det}\left[p_{I J}(s, t)\right]=0 \text {. }
$$

Equation (2.31) has no solutions unless (2.32) holds for every value of $s$ and $t$. Equation (2.32) is, of course, an extremely onerous condition, and we find that it is not met for the $p_{I J}$ matrix that arises in the study of $S$ matrices with $D \geq 5$. It follows, as a consequence, that the local module is also freely generated for $D \geq 5$.

In the case that $|L|>|B|$ (which we encounter for $D=4$ and also $D=3$ for photons), on the other hand, it is very easy to see that $|L|$ cannot be freely generated. ${ }^{42}$ Given that the local module is not freely generated in $D=4$, it is important to discover the relations in this module. We will return to this question later in this paper.

\footnotetext{
${ }^{40}$ The remarkable fact is that $p_{I J}(s, t)$ is simply a polynomial without any negative or fractional powers. Unfortunately we do not have a simple abstract proof of this statement, and it would be nice to find one. Nonetheless we have explicitly verified it in every dimension and for both photon and graviton scattering. The verification has proceeded on Mathematica; in each dimension we have used Mathematica to explicitly re express the local generators in terms of the bare generators and verified that the coefficients of this expansion are polynomials in $s, t, u$.

${ }^{41}$ The symbol $|A|$ denotes the number of elements in the set $A$.

${ }^{42}$ The argument for this is the following. If $L$ were freely generated then the number of local quasiinvariant S-matrices of degree $d$ would grow like $|L| d$ at large $d$. This is larger than the number of bare quasi-invariant S-matrices, which grows like $|B| d$ at large $d$, contradicting the fact that the local module is a submodule of the bare module.
} 


\subsection{Irreducible representations of $S_{3}$ and fusion rules}

As we have explained above, the space of physical (hence $S_{4}$ invariant) S-matrices is the projection of the local module of S-matrices onto $S_{3}$ singlets. In this subsection we discuss the nature of this projection. As preparation for our discussion we first review elementary facts about $S_{3}$ representation theory.

The permutation group of three elements, $S_{3}$, has three irreducible representations the one dimensional totally symmetric representation which we call $\mathbf{1}_{\mathbf{S}}$, the one dimensional totally anti-symmetric representation which we call $\mathbf{1}_{\mathbf{A}}$ and a two dimensional representation with mixed symmetry which we call $\mathbf{2}_{\mathbf{M}}$. The subscript for $\mathbf{2}_{\mathbf{M}}$ emphasizes the mixed symmetry. It is standard to associate the following Young diagrams with these representations,

$$
\mathbf{1}_{\mathbf{S}}=\square \square \quad \mathbf{1}_{\mathbf{A}}=\boxminus \quad \mathbf{2}_{\mathbf{M}}=\square .
$$

It is easy to decompose an representation of $S_{3}$, into the subspaces that transform, respectively, in the $\mathbf{1}_{\mathbf{S}}$, the $\mathbf{1}_{\mathbf{A}}$ and $\mathbf{2}_{\mathbf{M}}$ representations. Complete symmetrization project onto the $\mathbf{1}_{\mathrm{S}}$ subspace, complete anti symmetrization projects onto the $\mathbf{1}_{\mathrm{A}}$ subspace and whatever is left over, i.e. the part that is annihilated by both complete symmetrization and complete anti symmetrization, transforms in the $\mathbf{2}_{\mathbf{M}}$ representation.

In order to get some familiarity with these representations, let us first consider a 3 dimensional column vector whose elements are $q_{1}, q_{2}, q_{3}$ respectively. The permutation group has a natural action on this column vector; any given element $\sigma$ of $S_{3}$ maps this vector to the column with entries $q_{\sigma(1)}, q_{\sigma(2)}, q_{\sigma(3)} \cdot{ }^{43}$ This linear map is generated by a (unique) $3 \times 3$ matrix $M(\sigma)$ acting on acting on the column $\left(q_{1}, q_{2}, q_{3}\right)$. The collection of matrices $M(\sigma)$ yields a representation of $S_{3}$. We use the symbol 3 to denote this 'defining' representation of $S_{3}$. This representation is not irreducible but can be decomposed as ${ }^{44}$

$$
3=\mathbf{2}_{\mathrm{M}}+\mathbf{1}_{\mathrm{S}}
$$

As a second exercise let us study the 6 dimensional representation, $\mathbf{6}_{\text {left }}$ generated by the left action of $S_{3}$ onto itself. It is not difficult to demonstrate (see appendix B) that

$$
\mathbf{6}_{\mathrm{left}}=\mathbf{1}_{\mathrm{S}}+2 \cdot \mathbf{2}_{\mathrm{M}}+\mathbf{1}_{\mathbf{A}} .
$$

Of course the same decomposition also applies for the $\mathbf{6}_{\text {right }}$ representation generated by the right action of $S_{3}$ on itself.

As our next example consider the adjoint action of $S_{3}$ on itself $\sigma \rightarrow g^{-1} \sigma g$, which also yields a 6 dimensional representation $\boldsymbol{6}_{\text {adj }}$. The adjoint representation can be decomposed into the $\mathbf{1}_{\mathbf{S}}$ (which acts on the identity element which is invariant under adjoint action) a

\footnotetext{
${ }^{43}$ For instance the $\mathbb{Z}_{2}$ element that flips one and two gives $\left(q_{2}, q_{1}, q_{3}\right)$.

${ }^{44}$ To see why this is the case, note that the complete symmetrization the column $x_{1}, x_{2}, x_{3}$ yields a column whose elements are all equate to $2\left(x_{1}+x_{2}+x_{3}\right)$. This column is permutation invariant and so transforms in the one dimensional completely symmetric representation of $S_{3}$. Removing this column one is left with the action of $S_{3}$ on a column $\left(y_{1}, y_{2}, y_{3}\right)$ whose elements are subject to the constraint $y_{1}+y_{2}+y_{3}=0$, which generates the $\mathbf{2}_{\mathrm{M}}$ representation.
} 
$\mathbf{3}$ (which acts on the 3 exchange permutations (213), (321), (132)), and a $\mathbf{2}_{\mathbf{M}}$ (which acts on the two cyclical permutations (231) and (312)). In equations

$$
\mathbf{6}_{\mathrm{adj}}=\mathbf{1}_{\mathrm{S}}+\mathbf{2}_{\mathrm{M}}+\mathbf{3} .
$$

Of course the $\mathbf{3}$ can itself be further decomposed using (2.34).

The action of the adjoint representation on the permutation group is particularly important, because the elements that transform in the same representation in (2.36) have equal eigenvalues when acting on any representation. The identity element (symmetric representation in (2.36)), of course, acts as unity on every representation. The cyclic elements (which transform in the $\mathbf{2}_{\mathbf{M}}$ in (2.36)) obey the identity $g^{3}=1$. It follows that the eigenvalues of the cyclic elements are cube roots of unity in any representation of the symmetric group. It is not difficult to verify that each of these elements act as unity on the $\mathbf{1}_{\mathbf{S}}$ and $\mathbf{1}_{\mathbf{A}}$ representations but have eigenvalues $\left(e^{\frac{2 \pi i}{3}}, e^{-\frac{2 \pi i}{3}}\right)$ in the $\mathbf{2}_{\mathbf{M}}$ representation. Finally the exchange elements (which transform in the $\mathbf{3}$ in (2.36)) obey $g^{2}=1$ and so always have eigenvalue \pm 1 . These elements are represented by unity in the $\mathbf{1}_{\mathbf{S}}$, by -1 in the $\mathbf{1}_{\mathbf{A}}$, and have eigenvalues $(+1,-1)$ in the $\mathbf{2}_{\mathbf{M}}$ dimensional representation. Note also that the $\mathbf{2}_{\mathbf{M}}$ (cyclical elements) together with the identity make up the abelian group $\mathbb{Z}_{3}$ - the maximal abelian subgroup of $S_{3}$.

Note that it is possible to choose a basis of the $\mathbf{3}$ representation such that the three basis elements are respectively invariant under the three distinct exchange permutations. A converse of this statement is also true. Consider a representation vector that is invariant under (say) (213). Construct a set of three vectors given by this vector and its images under the two cyclic permutations. ${ }^{45}$ If the resultant set of 3 vectors are linearly independent then they transform in the $\mathbf{3}$ representation of $S_{3}$.

Recall that every $S_{3}$ element, so in particular every two particle exchange in $S_{3}$, is represented by unity in the $\mathbf{1}_{\mathbf{S}}$ representation. On the other hand every two particle exchange in $S_{3}$ is represented by -1 in the $\mathbf{1}_{\mathbf{A}}$ representation. Finally the $\mathbf{2}_{\mathbf{M}}$ representation somewhere right in between the $\mathbf{1}_{\mathbf{S}}$ and $\mathbf{1}_{\mathbf{A}}$ in the following sense: every two particle exchange element is represented by a $2 \times 2$ matrix whose eigenvalues are \pm 1 .

Second, given a collection of objects, $n_{\mathbf{1}_{\mathrm{S}}}$ of which transform in the completely symmetric representation, $n_{\mathbf{1}_{\mathbf{A}}}$ of which transform in the completely antisymmetric representation and $n_{\mathbf{2}_{\mathrm{M}}}$ of which transform in the mixed two dimensional representation. It is clear from the discussions of this subsection that our $n_{\mathbf{1}_{\mathbf{S}}}+n_{\mathbf{1}_{\mathbf{A}}}+2 n_{\mathbf{2}_{\mathbf{M}}}$ dimensional vector space of objects can be decomposed into a $n_{\mathbf{1}_{\mathbf{S}}}+n_{\mathbf{2}_{\mathrm{M}}}$ dimensional subspace in which any particular exchange operator, say (213), has eigenvalue plus one, and the complementary $n_{\mathbf{1}_{\mathbf{A}}}+n_{\mathbf{2}_{\mathbf{M}}}$ dimensional subspace in which the same exchange operator has eigenvalue -1 . It follows that the $\mathbb{Z}_{2}$ invariant subspace of our collection of objects is $n_{\mathbf{1}_{\mathrm{S}}}+n_{\mathbf{2}_{\mathrm{M}}}$ dimensional.

We end this subsection with a presentation of the fusion rules of $S_{3}$, i.e. the rules for the decomposition of the direct products of every pair of irreducible representations of $S_{3}$ into the sum of irreducible representations. It is clear that the direct product of any

\footnotetext{
${ }^{45}$ In more detail, the first basis element is invariant under (213) the second element under (321) and the third element under (132).
} 
representation $\mathbf{R}$ with the symmetric representation is $\mathbf{R}$. The remaining direct products of irreducible representations are easily verified to be (see appendix B),

$$
\mathbf{1}_{\mathrm{A}} \otimes \mathbf{2}_{\mathrm{M}}=\mathbf{2}_{\mathrm{M}}, \quad \mathbf{1}_{\mathrm{A}} \otimes \mathbf{1}_{\mathrm{A}}=\mathbf{1}_{\mathrm{S}}, \quad \mathbf{2}_{\mathrm{M}} \otimes \mathbf{2}_{\mathrm{M}}=\mathbf{2}_{\mathrm{M}} \oplus \mathbf{1}_{\mathrm{S}} \oplus \mathbf{1}_{\mathrm{A}}
$$

\subsection{Projecting onto $S_{3}$ singlets}

We now return to a discussion of the local module and its $S_{3}$ projection. It follows from definitions that every bare and local quasi-invariant S-matrix, denoted as $m\left(p_{i}, \epsilon_{i}\right)$ and $M\left(p_{i}, \epsilon_{i}\right)$ respectively, can be expressed as

$$
\begin{aligned}
m\left(p_{i}, \epsilon_{i}\right) & =\sum_{e_{I} \in B} p_{I}(s, t) e_{I}\left(\alpha_{i}, \epsilon_{i}^{\perp}\right) . \\
M\left(p_{i}, \epsilon_{i}\right) & =\sum_{E_{J} \in L} P_{J}(s, t) E_{J}\left(p_{i}, \epsilon_{i}\right) .
\end{aligned}
$$

where $p_{I}(s, t)$ and $P_{J}(s, t)$ are polynomials of $(s, t)$. The $S_{4}$ invariant local S-matrices are obtained by simply projecting the elements of the local module onto the trivial representation of $S_{3}$.

$$
\mathcal{S}\left(p_{i}, \epsilon_{i}\right)=\sum_{\sigma \in S_{3}} M^{\sigma}\left(p_{i}, \epsilon_{i}\right)=\sum_{E_{J} \in L} \sum_{\sigma \in S_{3}} P_{J}^{\sigma}(s, t) E_{J}^{\sigma}\left(p_{i}, \epsilon_{i}\right)
$$

The superscript $\sigma$ denotes the action of $\sigma$ permutation. As the local module admits the action of $S_{3}$, its generators $E_{J}$ 's can be decomposed into irreducible representations of $S_{3}$. Moreover the space of functions of $(s, t)$ can also be decomposed into irreducible representations of $S_{3}$. It follows from (2.39) that if a subset of $E_{J}$ 's transforms in any given irreducible representation $\mathbf{R}$ of $S_{3}$ then the functions $P_{J}(s, t)$ must also transform in the same representation $\mathbf{R} .^{46}$

In order to understand the detailed structure of the projection of the local module onto $S_{3}$ invariants we thus need to understand the decomposition of the space of polynomials of $(s, t)$ into representations of $S_{3}$. We turn to this question now.

\subsection{Action of $S_{3}$ on polynomials of $(s, t)$}

The action of $S_{3}$ on polynomials in $(s, t)$ is best realized as permutations of $(s, t, u)$, that belong to the defining representation 3 of $S_{3}$, subjected to the condition $s+t+u=0$. To gain some familiarity, let us work with some examples. To start with we ignore the constraint $s+t+u=0$ but impose it later.

At degree 0 , we only have 1 which clearly transforms in the symmetric representation. At degree 1, the space of polynomials is three dimensional, it decomposes into a symmetric and a mixed representation. The explicit form of the symmetric is $s+t+u$ and the two dimensional mixed representation is formed by $2 s-t-u$ and $2 t-s-u$. In order to verify the mixed symmetry, note that complete symmetrization or anti symmetrization of these polynomials vanish. At degree 2, the space of polynomials is 6 dimensional. It decomposes as two symmetric $s^{2}+t^{2}+u^{2}$ and $s t+t u+u s$ and two mixed representations. The mixed

\footnotetext{
${ }^{46}$ This conclusion follows from the fusion rules of $S_{3}$ listed in the previous subsection.
} 
representations are spanned by $\left(2 s^{2}-t^{2}-u^{2}, 2 t^{2}-s^{2}-u^{2}\right)$ and $(2 s t-t u-u s, 2 t u-s t-u s)$ respectively. The space of polynomials at degree 3 is 10 dimensional. It is decomposed as 3 symmetric, 3 mixed symmetric and 1 anti-symmetric. Here we will give explicit expression of the anti-symmetric only, $s^{2} t+t^{2} u+u^{2} s-s t^{2}-t u^{2}-u s^{2}$.

Some of the representations discussed above vanish after imposing momentum conservation $s+t+u=0$. At degree 1 , one mixed representation and at degree 2 , one symmetric and one mixed and at degree 3 , one symmetric, one mixed and one anti-symmetric survive.

It is useful to count these polynomials by their representations into a partition function. Again, we first ignore the constraint $s+t+u=0$. The single variable partition function is

$$
z(x):=\operatorname{Tr} x^{2 \Delta}=\frac{1}{1-x^{2}} .
$$

Here $\Delta$ is the degree of momentum homogeneity. The partition functions over polynomials of three variables with given transformation property are

$$
\begin{aligned}
\widetilde{Z}(x) & :=Z_{\mathrm{no-sym}}=z(x)^{3}, \quad \widetilde{Z}_{\mathbf{1}_{\mathbf{S}}, \mathbf{1}_{\mathbf{A}}}(x)=\frac{1}{6} z(x)^{3} \pm \frac{1}{2} z(x) z\left(x^{2}\right)+\frac{1}{3} z\left(x^{3}\right), \\
\widetilde{Z}_{\mathbf{2}_{\mathbf{M}}}(x) & =\frac{\widetilde{Z}(x)-\widetilde{Z}_{\mathbf{1}_{\mathbf{S}}}(x)-\widetilde{Z}_{\mathbf{1}_{\mathbf{A}}}(x)}{2} .
\end{aligned}
$$

The quantities $\widetilde{Z}_{\mathbf{R}}$ that appear in (2.41) are defined by

$$
\widetilde{Z}_{\mathbf{R}}=\sum_{m} n_{\mathbf{R}}(m) x^{2 m}
$$

where $n_{\mathbf{R}}(m)$ is the number of $S_{3}$ representations of type $\mathbf{R}$ that appear in the decomposition of polynomials of $s, t, u$ into representations of $S_{3}$ at degree $m .^{47}$

The space of polynomials in representation $\mathbf{R}$ without the constraint is generated by the space of polynomials in representation $\mathbf{R}$ with the constraint by multiplying it with polynomials of a single variable $s+t+u$. For partition functions it means,

$$
Z_{\mathbf{R}}(x)=\left(1-x^{2}\right) \widetilde{Z}_{\mathbf{R}}(x) .
$$

Here $Z_{\mathbf{R}}$ denotes the partition function over polynomials in the representation $\mathbf{R}$ with the constraint $s+t+u=0$. Using equations (2.41) and (2.43), we compute all the relevant partition functions. We find

$$
\begin{aligned}
Z_{\mathbf{1}_{\mathbf{S}}}(x) & =\mathrm{D}, \quad Z_{\mathbf{1}_{\mathbf{A}}}(x)=x^{6} \mathrm{D}, \quad Z_{\mathbf{2}_{\mathbf{M}}}(x)=\left(x^{2}+x^{4}\right) \mathrm{D}, \\
Z_{\mathbf{3}}(x) & =Z_{\mathbf{1}_{\mathbf{S}}}+Z_{\mathbf{2}_{\mathbf{M}}}(x)=\left(1+x^{2}+x^{4}\right) \mathrm{D} \\
Z_{\mathbf{3}_{\mathbf{A}}}(x) & =Z_{\mathbf{1}_{\mathbf{A}}}+Z_{\mathbf{2}_{\mathbf{M}}}(x)=\left(x^{6}+x^{2}+x^{4}\right) \mathrm{D} \\
Z_{\mathbf{6}}(x) & =Z_{\mathbf{1}_{\mathbf{S}}}+Z_{\mathbf{1}_{\mathbf{A}}}+2 Z_{\mathbf{2}_{\mathbf{M}}}(x)=\left(1+2 x^{2}+2 x^{4}+x^{6}\right) \mathrm{D} \\
\text { where } \quad \mathrm{D} & =\frac{1}{\left(1-x^{4}\right)\left(1-x^{6}\right)}
\end{aligned}
$$

(the representation $\mathbf{3}_{\mathbf{A}}$ is defined around (2.49) below).

\footnotetext{
${ }^{47}$ The factor of $1 / 2$ in th last of (2.41) follows from the fact that the $\mathbf{2}_{\mathrm{M}}$ representation is two dimensional; the partition function that counts representations is thus half the partition function that counts polynomials in this representation.
} 
The results (2.44) are easy to understand. The fully symmetric polynomials of $s, t$ and $u$ are arbitrary polynomials of the dimension 4 letter $s^{2}+t^{2}+u^{2}$ and the dimension 6 letter $s t u$. The lowest dimensional anti-symmetric polynomial occurs at dimension 6 and is given by $s^{2} t-t^{2} s-s^{2} u+s u^{2}-u^{2} t+t^{2} u$. The most general antisymmetric function is obtained by multiplying this element by an arbitrary polynomial of $s^{2}+t^{2}+u^{2}$ and $s t u$. Finally the two lowest doublets of mixed polynomials occur at dimension $2(s+t, s-t)$ and dimension $4\left(\left(s^{2}+t^{2}-2 u^{2}\right),\left(t^{2}+u^{2}-2 s^{2}\right)\right)$. The most general mixed polynomial is given by multiplying these doublets by an arbitrary polynomial of $s^{2}+t^{2}+u^{2}$ and $s t u$. These comments give a complete explanation of the formulae (2.44). See subsection 2.10 for a related discussion.

\subsection{Standard bases for representations of $S_{3}$}

Any function of $s, t$ and $u$ may be viewed as a function of $t, u$ only. The action of the permutation group on such functions generically produces several new functions. The set of $f(t, u)$ and all its permutations clearly transforms in a representation of the permutation group. The representations thus obtained differ depending on the symmetry properties of the function $f(t, u)$ that generate the orbit. We consider various cases in turn.

- If $f(t, u)=f(u, t)=f(-t-u, u)$ then the action of the permutation group leaves $f$ invariant. $f$ transforms in the $\mathbf{1}_{\mathbf{S}}$ representation of the permutation group.

- If $f(t, u)=-f(u, t)=-f(-t-u, u)$ then the permutation group acts on $f$ as $f \rightarrow \operatorname{sgn}(P) f$. In this case $f$ transforms in $\mathbf{1}_{\mathbf{A}}$ representation of the permutation group.

- If $f(t, u)=f(u, t)$ and

$$
f(t, u)+f(-t-u, t)+f(u,-t-u)=0
$$

Then the triplet

$$
\left(N^{\mathbf{2}_{\mathbf{M}},(1)}, N^{\mathbf{2}_{\mathrm{M}},(2)}, N^{\mathbf{2}_{\mathbf{M}},(3)}\right) \equiv(f(t, u), f(u, s), f(s, t))
$$

transforms in the $\mathbf{2}_{\mathbf{M}}$ representation. Note that

$$
\sum_{i} N^{\mathbf{2}_{\mathrm{M}},(i)}=0
$$

consistent with the fact that the $\mathbf{2}_{\mathbf{M}}$ representation is two dimensional. In the rest of this paper we will refer to (2.46) as the 'symmetric' basis for $\mathbf{2}_{\mathbf{M}}$ representation. Note the following property of the symmetric basis: the permutation $3 \leftrightarrow 4$ (equivalently $t \leftrightarrow u$ ) leaves the (1) basis element invariant. In a similar way the permutations $2 \leftrightarrow 4$ (resp. $2 \leftrightarrow 3$ ) leaves the (2) (resp.(3)) basis element invariant.

- If $f(t, u)=-f(u, t)$ and

$$
f(t, u)+f(-t-u, t)+f(u,-t-u)=0
$$


then the triplet

$$
\left(\widetilde{N}^{\mathbf{2}_{\mathrm{M}},(1)}, \widetilde{N}^{\mathbf{2}_{\mathrm{M}},(2)}, \widetilde{N}^{\mathbf{2}_{\mathrm{M}},(3)}\right) \equiv(f(t, u), f(u, s), f(s, t))
$$

also transforms in the $\mathbf{2}_{\mathbf{M}}$ representation. Note that once again

$$
\sum_{i} \widetilde{N}^{2_{\mathrm{M}},(i)}=0
$$

We will refer to (2.48) as the 'antisymmetric basis' for $\mathbf{2}_{\mathbf{M}}$ representation. In the antisymmetric basis the permutation $3 \leftrightarrow 4$ (equivalently $t \leftrightarrow u$ ) takes the (1) basis element to minus itself. In a similar way the permutations $2 \leftrightarrow 4$ (resp. $2 \leftrightarrow 3$ ) take the (2) (resp.(3)) basis elements to minus themselves.

- If $f(t, u)=f(u, t)$ but (2.45) is not obeyed then the triplet (2.46), which we now denote by

$$
\left(N^{\mathbf{3},(1)}, N^{\mathbf{3},(2)}, N^{\mathbf{3},(3)}\right) \equiv(f(t, u), f(u, s), f(s, t)),
$$

transforms in the $\mathbf{3}$ representation. Of course the $\mathbf{3}$ is no more than the sum of a $\mathbf{1}_{\mathbf{S}}$ — namely the quantity on the l.h.s. of $(2.45)$ - and a $\mathbf{2}_{\mathbf{M}}$ listed in the symmetric basis. Eq. (2.46) is the only basis we will use for the $\mathbf{3}$ representation.

- Similarly, if $f(t, u)=-f(u, t)$ but (2.47) is not obeyed then the triplet (2.48), which we now denote by

$$
\left(\widetilde{N}^{\mathbf{3}_{\mathbf{A}},(1)}, \widetilde{N}^{\mathbf{3}_{\mathbf{A}},(2)}, \widetilde{N}^{\mathbf{3}_{\mathbf{A}},(3)}\right) \equiv(f(t, u), f(u, s), f(s, t))
$$

transforms in the $\mathbf{3}_{\mathbf{A}}$ representation. The $\mathbf{3}_{\mathbf{A}}$ is no more than the sum of a $\mathbf{1}_{\mathbf{A}}$ namely the quantity on the l.h.s. of $(2.45)$ - and a $\mathbf{2}_{\mathrm{M}}$ in the antisymmetric basis. Eq. (2.50) is the only basis we will use for the $\mathbf{3}_{\mathbf{A}}$ representation.

- Finally, if $f(t, u)$ obeys no symmetry property whatsoever, then the orbit of the permutation group on $f$ is six dimensional; we obtain the $\boldsymbol{6}_{\text {left }}$ representation. One basis for this representation is obtained follows. We can write $f(t, u)$ as a sum of a symmetric and an antisymmetric function

$$
f(t, u)=\frac{f(t, u)+f(u, t)}{2}+\frac{f(t, u)-f(u, t)}{2}
$$

The symmetric part of $f$ then generates a $\mathbf{3}$ while the antisymmetric part generates a $\mathbf{3}_{\mathbf{A}}$. We can then use our standard basis for the $\mathbf{3}$ and $\mathbf{3}_{\mathbf{A}}$. This is indeed the strategy we will adopt when we need to choose a basis for the $\boldsymbol{6}_{\text {left }}$ representation. We emphasize, however, that unlike in every other case described above our basis for the $\boldsymbol{6}_{\text {left }}$ is ambiguous in the following manner. The decomposition of the $\boldsymbol{6}_{\text {left }}$ into the $\mathbf{3}$ and the $\mathbf{3}_{\mathbf{A}}$ is convention dependent. This can be understood in many ways. From a group theoretic viewpoint the grouping of the $\mathbf{1}_{\mathbf{S}}+2 \cdot \mathbf{2}_{\mathbf{M}}+\mathbf{1}_{\mathbf{A}}$ into a $\mathbf{1}_{\mathrm{S}}+\mathbf{2}_{\mathrm{M}}$ and a $\mathbf{1}_{\mathrm{A}}+\mathbf{2}_{\mathrm{M}}$ is inherently ambiguous, as there is no canonical way of choosing to associate one of the two $\mathbf{2}_{\mathbf{M}}$ 's with the $\mathbf{1}_{\mathbf{S}}$ and the other $\mathbf{2}_{\mathbf{M}}$ with the 
$\mathbf{1}_{\mathbf{A}}$. Given one such grouping, we can as well replace the two $\mathbf{2}_{\mathbf{M}}$ 's by any linearly independent linear combination of the two. From the practical constructive point of view the procedure outlined above has a degree of arbitrariness because the basis we obtain depends not just on the orbit of functions obtained from $f(t, u)$ but also on a choice of a particular function in the orbit (in this case $f(t, u)$ itself, rather than, for instance, $f(u, s)$ to get the procedure going). Different choices of starting functions differ in that they group different linear combinations of the two independent $\mathbf{2}_{\mathbf{M}}$ 's with the $\mathbf{1}_{\mathbf{S}}$ and $\mathbf{1}_{\mathbf{A}}$. When we need a basis for objects that transform in the $\mathbf{6}_{\text {left }}$ below we will indeed decompose this representation into the $\mathbf{3}$ and $\mathbf{3}_{\mathbf{A}}$, but will be very careful to explicitly spell out the particular conventions that give meaning to this decomposition.

To end this subsection we present the explicit computation of a particular ClebschGordon coefficient. Recall that the $\mathbf{2}_{\mathrm{M}} \times \mathbf{2}_{\mathrm{M}}=\mathbf{1}_{\mathrm{S}}+\mathbf{1}_{\mathrm{A}}+\mathbf{2}_{\mathrm{M}}$. Working with two copies of the $\mathbf{2}_{\mathrm{M}}$ in the symmetric basis we will now work out the explicit linear combination of products of original basis elements that transform in the $\mathbf{1}_{\mathbf{A}}$. Let

$$
\left(N_{1}^{\mathbf{2}_{\mathrm{M}},(1)}, N_{1}^{\mathbf{2}_{\mathrm{M}},(2)}, N_{1}^{\mathbf{2}_{\mathrm{M}},(3)}\right), \quad\left(N_{2}^{\mathbf{2}_{\mathrm{M}},(1)}, N_{2}^{\mathbf{2}_{\mathrm{M}},(2)}, N_{2}^{\mathbf{2}_{\mathrm{M}},(3)}\right)
$$

represent two distinct vectors that transform in the symmetric representation of the $\mathbf{2}_{\mathbf{M}}$ basis. Then it is not difficult to verify that

$N_{1}^{\mathbf{2}_{\mathrm{M}},(1)}\left(N_{2}^{\mathbf{2}_{\mathrm{M},(2)}}-N_{2}^{\mathbf{2}_{\mathrm{M},(3)}}\right)+N_{1}^{\mathbf{2}_{\mathrm{M}},(2)}\left(N_{2}^{\mathbf{2}_{\mathrm{M},(3)}}-N_{2}^{\mathbf{2}_{\mathrm{M},(1)}}\right)+N_{1}^{\mathbf{2}_{\mathrm{M}},(3)}\left(N_{2}^{\mathbf{2}_{\mathrm{M}},(1)}-N_{2}^{\mathbf{2}_{\mathrm{M},(2)}}\right)$

transforms in the $\mathbf{1}_{\mathbf{A}}$ representation. ${ }^{48}$

\subsection{Counting S-matrices}

As we have seen, four particle S-matrices are always characterized by an infinite number of parameters. However the number of parameters that appears at any given derivative

\footnotetext{
${ }^{48}$ One quick way to see this is to specialize to the particular case in which the two abstract triplets above
} are triplets of functions. Suppose the two $\mathbf{2}_{\mathrm{M}} s$ in symmetric basis be

$$
\left(f_{1}(t, u), f_{1}(s, u), f_{1}(u, t)\right), \quad\left(f_{2}(t, u), f_{2}(s, u), f_{3}(u, t)\right)
$$

(where all functions are symmetric in their arguments as appropriate for the symmetric basis). It is trivial to verify that the function

$$
f_{1}(u, t)\left(f_{2}(s, u)-f_{2}(t, s)\right)+f_{1}(s, u)\left(f_{2}(t, s)-f_{2}(t, u)\right)+f_{1}(t, s)\left(f_{2}(t, u)-f_{2}(s, u)\right)
$$

is completely antisymmetric in its arguments and so transforms in the $\mathbf{1}_{\mathbf{A}}$ representation.

The function in (2.53) is antisymmetric.

It follows that

$$
N_{1}^{\mathbf{3},(1)}\left(N_{2}^{\mathbf{2}_{\mathrm{M}},(2)}-N_{2}^{\mathbf{2}_{\mathbf{M}},(3)}\right)+N_{1}^{\mathbf{3},(2)}\left(N_{2}^{\mathbf{2}_{\mathbf{M}},(3)}-N_{2}^{\mathbf{2}_{\mathbf{M}},(1)}\right)+N_{1}^{\mathbf{3},(3)}\left(N_{2}^{\mathbf{2}_{\mathbf{M}},(1)}-N_{2}^{\mathbf{2}_{\mathbf{M}},(2)}\right)
$$

and

$$
N_{1}^{\mathbf{3},(1)}\left(N_{2}^{\mathbf{3},(2)}-N_{2}^{\mathbf{3},(3)}\right)+N_{1}^{\mathbf{3},(2)}\left(N_{2}^{\mathbf{3},(3)}-N_{2}^{\mathbf{3},(1)}\right)+N_{1}^{\mathbf{3},(3)}\left(N_{2}^{\mathbf{3},(1)}-N_{2}^{\mathbf{3},(2)}\right)
$$

also transform in the $\mathbf{1}_{\mathrm{A}}$ representation of $S_{3}$. 
order is finite. For this reason it is useful to characterize the data needed to specify an $S$ matrix by a partition function

$$
Z_{\mathrm{S}-\text { matrix }}(x)=\sum_{m=0}^{\infty} n(m) x^{m}
$$

where $n(m)$ is the number of free parameters that appear in the most general polynomial S-matrix at $m$ derivative order. ${ }^{49}$

The local module structure presented above makes the computation of $Z_{\mathrm{S} \text {-matrix }}(x)$ rather simple. Let us first consider the case of both graviton and photon scattering in $D \geq 5$. As we have mentioned above, in this case the local modules are freely generated. The generators of these modules, $E_{J}^{\sigma}$, are each characterized by their derivative dimension $\Delta_{J}$ and their $S_{3}$ representation $\mathbf{R}_{\mathbf{J}}$. It follows from (2.39) that in this case $Z_{\mathrm{S} \text {-matrix }}(x)$ is given by

$$
Z_{\mathrm{S}-\operatorname{matrix}}(x)=\sum_{J} x^{\Delta_{J}} Z_{\mathbf{R}_{\mathbf{J}}}(x)
$$

where $Z_{\mathbf{R}_{\boldsymbol{J}}}(x)$ are listed in (2.44).

Let us now turn to the case $D \leq 4$. In this case the local Modules are not freely generated, but instead have relations. As we will see below it turns out that the relation modules are all themselves free (there are no relations for relations). Let the generators for the relations be labelled by their derivative dimension $\Delta_{I}$ and $S_{3}$ representation $\mathbf{R}_{\mathbf{I}}$. It follows that in $D \leq 4$ the formula (2.58) is modified to

$$
Z_{\text {S-matrix }}(x)=\sum_{J} x^{\Delta_{J}} Z_{\mathbf{R}_{J}}(x)-\sum_{I} x^{\Delta_{I}} Z_{\mathbf{R}_{\mathbf{I}}}(x)
$$

where the sum over $J$ runs over all module generators, while the sum over $I$ runs over all relation generators.

\subsection{Regge growth}

Recall that the generators of the bare module are zero order in derivatives in the parity even sector, and also in the parity odd sector for even $D$. In these cases the generators are functions of $\alpha_{i}, \epsilon_{i}^{\perp}$ but are not separately functions of $s, t$ and $u$. On the other hand when $D$ is odd, the parity odd generators are proportional to $\sqrt{s t u}$ times functions of $\alpha_{i}, \epsilon_{i}^{\perp}$. In order to deal uniformly with all cases below, we introduce the variable $a$; $a=0$ for parity even S-matrices in every $D$ and parity odd S-matrices in even $D . a=3$ for parity odd S-matrices in odd $D$.

We will now derive a lower bound for the Regge growth for local S-matrices at $2 n+a$ order in derivatives. In order to do this we note that every such S-matrix is an $n^{\text {th }}$ order descendant of some bare generator. The generator in question might transform in the $\mathbf{1}_{\mathbf{S}}$, the $\mathbf{1}_{\mathrm{A}}$ or the $\mathbf{2}_{\mathrm{M}}$ representation, or a linear combination of these. We take these cases up in turn.

\footnotetext{
${ }^{49}$ In computing the derivative order we assign $\epsilon_{\mu}$ dimension zero.
} 
Consider any bare generator, say $\left|e_{\mathbf{S}}\right\rangle$, that transforms in the $\mathbf{1}_{\mathbf{S}}$. As explained under (2.44), the most general $n^{\text {th }}$ level descendant of this generator that itself transforms in the $\mathbf{1}_{\mathrm{S}}$ representation is given, for $n \geq 2$, by

$$
\left(\sum_{k, m} a_{k, m}(s t u)^{k}\left(s^{2}+t^{2}+u^{2}\right)^{m}\right)\left|e_{\mathbf{S}}\right\rangle
$$

where the sum runs over all terms with $3 k+2 m=n$. It is easy to convince oneself that all the S-matrices in (2.59) grow at least as fast as

$$
s^{\left(2\left[\frac{n+2}{3}\right]+\frac{a}{3}\right)}
$$

in the Regge limit. ${ }^{50}$ where $[m]$ represents the largest integer no smaller than $m$.

Now consider a bare generator $\left|e_{\mathbf{A}}\right\rangle$ that transforms in the antisymmetric representation. The most general descendant at $2 n+a$ order in derivatives is given by

$$
\left(s^{2} u-u^{2} s+t^{2} s-t^{2} u-s^{2} t+u^{2} t\right)\left(\sum_{k, m} a_{k, m}(s t u)^{k}\left(s^{2}+t^{2}+u^{2}\right)^{m}\right)\left|e_{\mathbf{A}}\right\rangle
$$

where $3 k+2 m=n-3$. For $n=3$ and $n \geq 5$ all terms in (2.61) grow at least as fast in the Regge limit as, ${ }^{51}$

$$
s^{\left(2\left[\frac{n-1}{3}\right]+3+\frac{a}{3}\right) .}
$$

Finally consider a bare generator multiplet that transforms in the $\mathbf{2}_{\mathrm{M}}$ representation. Let the triplet of basis vectors

$$
\left(\left|e_{\mathbf{M}}^{(1)}\right\rangle,\left|e_{\mathbf{M}}^{(2)}\right\rangle,\left|e_{\mathbf{M}}^{(3)}\right\rangle\right)
$$

transform in the $\mathbf{2}_{\mathbf{M}}$ representation in the symmetric basis (see around (2.46)). ${ }^{52}$ The most general $2 n+a$ derivative descendant of these basis vectors is given either by

$$
\left(\sum_{k, m} a_{k, m}(s t u)^{k}\left(s^{2}+t^{2}+u^{2}\right)^{m}\right)\left(s\left|e_{\mathbf{M}}^{(1)}\right\rangle+t\left|e_{\mathbf{M}}^{(2)}\right\rangle+u\left|e_{\mathbf{M}}^{(3)}\right\rangle\right)
$$

with $3 k+2 m=n-1$ or by

$$
\begin{aligned}
& \left(\sum_{k, m} a_{k, m}(s t u)^{k}\left(s^{2}+t^{2}+u^{2}\right)^{m}\right) \\
& \quad \times\left(\left(t^{2}+u^{2}-2 s^{2}\right)\left|e_{\mathbf{M}}^{(1)}\right\rangle+\left(u^{2}+s^{2}-2 t^{2}\right)\left|e_{\mathbf{M}}^{(2)}\right\rangle+\left(s^{2}+t^{2}-2 u^{2}\right)\left|e_{\mathbf{M}}^{(3)}\right\rangle\right)
\end{aligned}
$$

\footnotetext{
${ }^{50}$ When $n=3 p$, we obtain the slowest growth when $a_{k, m}$ is non-zero only for $k=p$ and $m=0$. When $n=3 p+1$ (and $p \geq 1$ ) the slowest growth is achieved when $a_{k, m}$ is non-zero only when $k=p-1$ and $m=2$. When $n=3 p+2$ we get the slowest growth for the monomial with $k=p$ and $m=1$.

${ }^{51}$ When $n=3 p$, we obtain the slowest growth when $a_{k, m}$ is non-zero only for $k=p-1$ and $m=0$. When $n=3 p+1$ (and $p \geq 2$ ) the slowest growth is achieved when $a_{k, m}$ is non-zero only when $k=p-2$ and $m=2$. When $n=3 p+2$ we get the slowest growth for the monomial with $k=p-1$ and $m=1$.

${ }^{52}$ Note in particular that

$$
\left|e_{\mathbf{M}}^{(1)}\right\rangle+\left|e_{\mathbf{M}}^{(2)}\right\rangle+\left|e_{\mathbf{M}}^{(3)}\right\rangle=0 .
$$
}


with $3 k+2 m=n-2$. All the S-matrices in (2.63) and (2.64) grow at least as fast in the Regge limit as ${ }^{53}$

$$
\begin{array}{lll}
s^{\alpha+\frac{a}{3}} & & \\
\alpha=2 p+1 & \text { when } & n=3 p \\
\alpha=2 p+1 & \text { when } & n=3 p+1 \\
\alpha=2 p+2 & \text { when } & n=3 p+2
\end{array}
$$

Combining all the results above we conclude that every local S-matrix at $2 n+a$ derivative order grows at least as fast in the Regge limit as

$$
\begin{array}{lll}
s^{\alpha(n)+\frac{a}{3}} & & \\
\alpha(n)=2 p & \text { when } & n=3 p \\
\alpha(n)=2 p+1 & \text { when } & n=3 p+1 \\
\alpha(n)=2 p+2 & \text { when } & n=3 p+2
\end{array}
$$

The bound in the first line in the first line in (2.66) is saturated by the state $(s t u)^{p}\left|e_{\mathbf{S}}\right\rangle$, the bound in the second line is saturated by the state

$$
(s t u)^{p}\left(s\left|e_{\mathbf{M}}^{(1)}\right\rangle+t\left|e_{\mathbf{M}}^{(2)}\right\rangle+u \mid e_{\mathbf{M}}^{(3)}\right)
$$

and the bound in the third line in (2.66) is saturated both by $(s t u)^{p}\left(s^{2}+t^{2}+u^{2}\right)\left|e_{\mathbf{S}}\right\rangle$ and by

$$
(s t u)^{p}\left(\left(t^{2}+u^{2}-2 s^{2}\right)\left|e_{\mathbf{M}}^{(1)}\right\rangle+\left(u^{2}+s^{2}-2 t^{2}\right)\left|e_{\mathbf{M}}^{(2)}\right\rangle+\left(s^{2}+t^{2}-2 u^{2}\right)\left|e_{\mathbf{M}}^{(3)}\right\rangle\right) .
$$

As we have mentioned in the introduction, we are particularly interested in local Smatrices that grow no faster than $s^{2}$ in the Regge limit. We end this section with a complete listing of all module elements that have this feature. For parity even S-matrices — or parity odd S-matrices in even $D$ these possibilities are

- At zero order in derivatives generators of the bare module in the $\mathbf{1}_{\mathbf{S}}$ representation yield S-matrices that grow like $s^{0}$ in the Regge limit.

- At the two derivative level we have S-matrices of the form (2.63) with $a_{k, m}$ non-zero only when $k=m=0$. These S-matrices grow like $s$ in the Regge limit.

- At fourth order in derivatives we have S-matrices of the form (2.59) with $a_{k, m}=0$ unless $k=0, m=1$. We also have S-matrices of the form (2.64) with $a_{k, m}=0$ unless $k=m=0$. These S-matrices grow like $s^{2}$ in the Regge limit.

- At six derivative order the unique such S-matrix is of the form (2.59) with $a_{k, m}=0$ unless $k=1$ and $m=0$.

\footnotetext{
${ }^{53}$ When $n=3 p$, we obtain the slowest growth from the term in (2.63) with $k=p-1$ and $m=1$. When $n=3 p+1$ the slowest growth comes from the term in (2.63) with $k=p$ and $m=0$. When $n=3 p+2$ we get the slowest growth for the monomial in (2.64) with $k=p$ and $m=0$.
} 
All other local S-matrices - in particular all S-matrices that are of 8 or higher order in derivatives - necessarily grow faster than $s^{2}$ in the Regge limit.

In the case of parity odd S-matrices in odd $D$, the only Module elements that grow no faster than $s^{2}$ in the Regge limit are

- At 3 derivative order we have generators of the bare module in the $\mathbf{1}_{\mathbf{S}}$ representation. The corresponding S-matrices grow like $s$ in the Regge limit.

- At the five derivative level we have S-matrices of the form (2.63) with $a_{k, m}$ non-zero only when $k=m=0$. These S-matrices grow like $s^{2}$ in the Regge limit.

All other S-matrices - in particular all S-matrices of 7 or higher order in derivatives grow faster than $s^{2}$ in the Regge limit.

\section{Generators of the bare module}

In this section we will enumerate and explicitly construct $e_{I}\left(\alpha_{i}, \epsilon_{i}^{\perp}\right)$, the basis of the bare module defined in subsection 2.4.2, both for four photon scattering and for four graviton scattering in every spacetime dimension. We also keep track of the $S_{3}$ transformation properties of $e_{I}$. This section is devoted purely to the study of the bare module. We postpone the study of the embedding of the local module into the bare module that we construct in this subsection to later in this paper, beginning with the next section in which we use a systematic plethystic program to enumerate the analytic index structures $E_{J}\left(p_{i}, \epsilon_{i}\right)$.

\subsection{Enumeration}

In this subsection we count the rank of the bare module, i.e. the number of linearly independent basis elements $e_{I}$. As explained in subsection 2.4.2, these are simply the set of $\mathrm{SO}(D-3)$ and $\mathbb{Z}_{2} \times \mathbb{Z}_{2}$ invariant polynomials of $\alpha_{i}$ and $\epsilon_{i}^{\perp}$ with the appropriate homogeneity properties. We separately enumerate parity odd and parity even generators of the bare module. As photons/gravitons have no propagating degrees of freedom when $D \leq 2$ and $D \leq 3$ respectively, we restrict our attention to $D \geq 3$ for photons $D \geq 4$ for gravitons. In the next subsection we will proceed to actually construct these basis elements $\left(e_{I}\right)$ and list their $S_{3}$ transformation properties.

Under $\mathrm{SO}(D-3)$, the effective polarization for photon takes values in the space $\rho=$ $(\mathrm{s} \oplus \mathrm{v}$ ) (for gravitons, $\rho=(\mathrm{s} \oplus \mathrm{v} \oplus \mathrm{t})) .{ }^{54}$ Here $\mathrm{s}, \mathrm{v}, \mathrm{t}$ are scalar, vector and symmetric traceless tensor of $\mathrm{SO}(D-3)$ respectively. The number of index structures is the number of singlets in

$$
\left.\rho^{\otimes 4}\right|_{\mathbb{Z}_{2} \times \mathbb{Z}_{2}} \text { : i.e. }\left.\quad(\mathbf{s} \oplus \mathbf{v})^{\otimes 4}\right|_{\mathbb{Z}_{2} \times \mathbb{Z}_{2}} \text { for photons, }\left.\quad(\mathbf{s} \oplus \mathbf{v} \oplus \mathbf{t})^{\otimes 4}\right|_{\mathbb{Z}_{2} \times \mathbb{Z}_{2}} \text { for gravitons }
$$

where the notation $\left.\right|_{G}$ stands for projection onto $G$ invariants.

\footnotetext{
${ }^{54}$ As we have explained above, photon $S$ matrices are separately linear in each of $\left(\epsilon_{i}^{\perp}, \alpha_{i}\right)$. Here $\epsilon_{i}^{\perp}$ is the v while $\alpha_{i}$ is the s. On the other hand gravitational S-matrices are quadratic separately in each of $\left(\epsilon_{i}^{\perp}, \alpha_{i}\right)$; and are evaluated subject to the constraint $\epsilon_{i}^{\perp} \cdot \epsilon_{i}^{\perp}+\alpha_{i}^{2}=0$. The terms $\epsilon_{i}^{\perp} \epsilon_{i}^{\perp}$ is the $\mathrm{t}$ above (this term is effectively traceless as the constraint (2.15) allows us to trade its trace for $\alpha_{i}^{2}$ ), the terms $\alpha_{i} \epsilon_{i}^{\perp}$ is the v and the terms $\alpha_{i}^{2}$ are the $\mathbf{s}$.
} 


\begin{tabular}{|l|l|l|}
\hline photons & even & odd \\
\hline$D \geq 8$ & 7 & 0 \\
\hline$D=7$ & 7 & 1 \\
\hline$D=6$ & 7 & 1 \\
\hline$D=5$ & 7 & 0 \\
\hline$D=4$ & 5 & 2 \\
\hline$D=3$ & 1 & 1 \\
\hline
\end{tabular}

\begin{tabular}{|l|l|l|}
\hline gravitons & even & odd \\
\hline$D \geq 8$ & 29 & 0 \\
\hline$D=7$ & 29 & 7 \\
\hline$D=6$ & 28 & 9 \\
\hline$D=5$ & 22 & 3 \\
\hline$D=4$ & 5 & 2 \\
\hline$D=3$ & - & - \\
\hline
\end{tabular}

Table 3. Number of parity even and parity odd index structures for 4-photon and 4-graviton S-matrix as various dimensions.

In order to perform the necessary enumeration we use the formula

$$
\left.\rho^{\otimes 4}\right|_{\mathbb{Z}_{2} \times \mathbb{Z}_{2}}=\rho^{4} \ominus 3\left(S^{2} \rho \otimes \wedge^{2} \rho\right) .
$$

where $S^{2} \rho$ and $\wedge^{2} \rho$ stand for the symmetric and antisymmetric square of $\rho$ respectively. Eq. (3.2) was derived and employed in [26] to study a closely related problem, namely the enumeration of inequivalent tensor structures in CFT four point functions. We present a simple 'physics' derivation of (3.2) in appendix C.

It follows from (3.2) that the dimensionality of $\left.\rho^{\otimes 4}\right|_{\mathbb{Z}_{2} \times \mathbb{Z}_{2}}$ can be enumerated by employing the following simple algorithm. Let $n_{a}^{\mathbf{1}} \mathrm{s}$ and $n_{a}^{\mathbf{1} \mathrm{A}}$ respectively represent the number of copies of the $\mathrm{SO}(D-3)$ representation $a$ that appear in the symmetric and antisymmetric square of $\rho$. Then the number of $\mathrm{SO}(D-3)$ singlets in the $\mathbb{Z}_{2} \times \mathbb{Z}_{2}$ invariant tensor product of four copies of $\rho$ is given by,

$$
\sum_{a}\left(\left(n_{a}^{\mathbf{1}} \mathbf{s}+n_{a}^{\mathbf{1}}\right)^{2}-3 n_{a}^{\mathbf{1}} \mathbf{s} n_{a}^{\mathbf{1}} \mathbf{A}\right)=\sum_{a}\left(\left(n_{a}^{\mathbf{1}} \mathbf{S}-n_{a}^{\mathbf{1}} \mathbf{A}\right)^{2}+n_{a}^{\mathbf{1}_{\mathbf{s}}} n_{a}^{\mathbf{1} \mathbf{A}}\right) .
$$

Using (3.3) the enumeration of simultaneous $\mathbb{Z}_{2} \times \mathbb{Z}_{2}$ and $\mathrm{SO}(D-3)$ invariants is straightforward. We have tabulated the result, in every dimension, in table 3. Even though the counting based on (3.3) only yields the total number of simultaneous $\mathrm{SO}(D-3)$ and $\mathbb{Z}_{2} \times \mathbb{Z}_{2}$ singlets, in table 3 we have anticipated the results of the rest of this section (see subsection 3.2 and appendix D) to separate this total into the separate contribution of even and odd structures.

Note that the number of parity even structures do not depend on dimension for $D \geq 7$. This is because the most general configuration of four transverse polarizations $\epsilon_{i}^{\perp}$ can always be rotated to be in a specified 4 dimensional hyperplane orthogonal to the scattering plane - say the hyperplane spanned by the directions $3,4,5,6$ if the scattering plane consists of the directions $0,1,2$. It follows that the most general scattering kinematics can be achieved if the number of spacetime dimensions is $3+4=7$. Any additional dimensions merely play the role of a spectator.

The analysis of the previous paragraph does not apply to parity odd amplitudes as they depend on $\varepsilon$. The reason that the number of parity odd amplitudes stabilizes (to zero) 
for $D \geq 8$ may be understood as follows. Parity odd S-matrices are linear in the LeviCivita tensor $\varepsilon$. Lorentz invariance requires that all free indices of $\varepsilon$ are contracted. As $\varepsilon$ is completely antisymmetric any given vector can contract with only one index of $\varepsilon$. As four particle scattering amplitudes are functions of 7 independent vectors (the four polarization vectors $\epsilon_{i}$ and the three independent scattering momenta), parity odd S-matrices do not exist in $D \geq 8$, but do exist in $D=7$, see table 3 .

As on-shell inequivalent AdS bulk Lagrangians give rise to distinct boundary correlators, the counting of graviton four point structures in $D$ dimensions is the same as the counting of index structures for stress tensor four point functions in a $D-1$ dimensional conformal field theory. This counting for parity even structures was performed in [27]. It agrees with the counting presented here.

\subsection{Explicit construction of $e_{I}$ and $S_{3}$ transformations}

In this subsection we explicitly construct the basis elements $e_{I}\left(\alpha_{i}, \epsilon_{i}^{\perp}\right)$ and thereby obtain their $S_{3}$ transformation properties. Our construction is motivated by construction of index structure for CFT four point functions in [28]. In the main text we present the details of our construction only for $D \geq 7$ and simply tabulate our final representation wise counting results in other dimensions. Detailed construction for $D<7$ are presented in appendix D.

The parity even and odd generators for photons will be denoted by the letters $e$ and $o$ respectively. We will label the structures with the $S_{3}$ representation they transform under. For example, a parity even photon generator transforming in $\mathbf{3}$ will be denoted as $e_{\mathbf{3}}$. If there are multiple of them then we also include an arbitrarily assigned serial number in the subscript. We will only need to concern ourselves with $\mathbf{1}_{S}, \mathbf{1}_{A}, \mathbf{3}$ and $\mathbf{6}_{\text {left }}=\mathbf{3} \oplus \mathbf{3}_{\mathbf{A}}$ representation. We always choose to work in standard representations (2.48) and (2.49) for 3 and $\mathbf{3}_{\mathbf{A}} \cdot{ }^{55}$ We will also include the space-time dimension in the superscript when it needs to be emphasized. This helps especially in the case of parity odd structures which crucially depend on space-time dimensions.

Let us first consider the case of parity even structures for photons. The structures will be labelled by the $S_{3}$ representation they transform under.

In this case $\left.\rho^{\otimes 4}\right|_{\mathbb{Z}_{2} \times \mathbb{Z}_{2}}$ has 7 distinct basis elements (see table 3). Keeping in mind that $\rho=\mathrm{s}+\mathrm{v}$, it follows that these 7 structures each have their origin in one (and only one) of the tensor products ${ }^{56}$

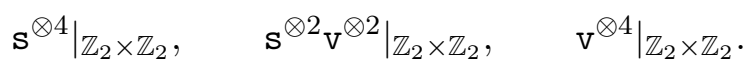

A slight generalization of the enumeration method described in the previous subsection and appendix $\mathrm{C}$ allows us to separately enumerate the basis elements in each of these sectors. We find that there is one element in $\left.\mathbf{s}^{\otimes 4}\right|_{\mathbb{Z}_{2} \times \mathbb{Z}_{2}}$ and three each in $\left.\mathbf{s}^{\otimes 2} \mathrm{v}^{\otimes 2}\right|_{\mathbb{Z}_{2} \times \mathbb{Z}_{2}}$ and $\left.\mathrm{v}^{\otimes 4}\right|_{\mathbb{Z}_{2} \times \mathbb{Z}_{2}}$.

\footnotetext{
${ }^{55_{1}}$.e, the basis of structure transforming in $\mathbf{3}$ (resp. $\mathbf{3}_{\mathbf{A}}$ ) such that state (1) is $\mathbb{Z}_{2}$ symmetric (resp. antisymmetric) under the swap $1 \leftrightarrow 2$. States (2) and (3) are obtained from (1) by cyclic permutation $234 \rightarrow 342$.

${ }^{56}$ The tensor products $\mathbf{s}^{\otimes 3} \mathrm{v}$ and $\mathrm{v}^{\otimes 3} \mathbf{s}$ do not contribute as they contain no $\mathrm{SO}(D-3)$ singlets.
} 


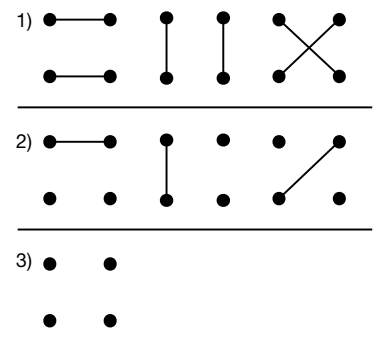

Figure 1. Parity even index structures of 4-photon scattering.

It is easy to explicitly construct these basis elements. Consider, for example, the sector $\left.\mathrm{v}^{\otimes 4}\right|_{\mathbb{Z}_{2} \times \mathbb{Z}_{2}}$. The 3 basis elements in this sector are

$$
e_{\mathbf{3}, 1}^{(1)}=\left(\epsilon_{1}^{\perp} \cdot \epsilon_{2}^{\perp}\right)\left(\epsilon_{3}^{\perp} \cdot \epsilon_{4}^{\perp}\right), \quad e_{\mathbf{3}, 1}^{(2)}=\left(\epsilon_{3}^{\perp} \cdot \epsilon_{1}^{\perp}\right)\left(\epsilon_{2}^{\perp} \cdot \epsilon_{4}^{\perp}\right) \quad e_{\mathbf{3}, 1}^{(3)}=\left(\epsilon_{2}^{\perp} \cdot \epsilon_{3}^{\perp}\right)\left(\epsilon_{1}^{\perp} \cdot \epsilon_{4}^{\perp}\right)
$$

It is easy to check that these structures are $\mathbb{Z}_{2} \times \mathbb{Z}_{2}$ invariant as desired. Also, each of the three elements listed in (3.5) happens to be invariant under a single $\mathbb{Z}_{2}$ exchange transformation; ${ }^{57}$ moreover the three elements are mapped to each other by the action of the cyclic elements of $S_{3} \cdot{ }^{58}$ It follows from the discussion in the second last paragraph of subsection 2.5 that these elements transform in the $\mathbf{3}$ representation of $S_{3}$ defined in and around (2.34).

It is useful to introduce a graphical notation to denote structures such as those explicitly presented in (3.5). Consider a graph with four vertices, one corresponding to each scattering particle. A line between any two vertices denotes the contraction of an $\epsilon^{\perp}$ at the corresponding vertices; for instance a line between vertex 1 and vertex 2 signifies the term $\epsilon_{1}^{\perp} . \epsilon_{2}^{\perp}$; two lines between the same two vertices represents two factors of this dot product. ${ }^{59}$ Factors of $\alpha_{i}$ are not explicitly denoted in the graph, but are inserted into the corresponding expressions to saturate homogeneity. With these conventions, the three expressions in (3.5) are represented by the three graphs on the first line of 1 . On the other hand the three structures that lie in the $\left.\mathbf{s}^{\otimes 2} \mathrm{v}^{\otimes 2}\right|_{\mathbb{Z}_{2} \times \mathbb{Z}_{2}}$ sector are the $\mathbb{Z}_{2} \times \mathbb{Z}_{2}$ symmetrized versions of the graphs listed in the second line of figure 1 . After $\mathbb{Z}_{2} \times \mathbb{Z}_{2}$ symmetrization, the algebraic expressions corresponding to the figures in this second line are

$$
\begin{aligned}
& e_{\mathbf{3}, 2}^{(1)}=\left(\epsilon_{1}^{\perp} \cdot \epsilon_{2}^{\perp} \alpha_{3} \alpha_{4}+\epsilon_{3}^{\perp} \cdot \epsilon_{4}^{\perp} \alpha_{1} \alpha_{2}\right), \quad e_{\mathbf{3}, 2}^{(2)}=\left(\epsilon_{1}^{\perp} \cdot \epsilon_{3}^{\perp} \alpha_{2} \alpha_{4}+\epsilon_{2}^{\perp} \cdot \epsilon_{4}^{\perp} \alpha_{1} \alpha_{3}\right), \\
& e_{\mathbf{3}, 2}^{(3)}=\left(\epsilon_{2}^{\perp} \cdot \epsilon_{3}^{\perp} \alpha_{1} \alpha_{4}+\epsilon_{1}^{\perp} \cdot \epsilon_{4}^{\perp} \alpha_{2} \alpha_{3}\right)
\end{aligned}
$$

As in the case of (3.5), the expressions in (3.6) are each invariant under a single $\mathbb{Z}_{2}$ exchange element and are also mapped to each other by the action of $\mathbb{Z}_{3}$. It thus follows that the expressions in (3.6) - like those in (3.5) - transform in the $\mathbf{3}$ representation of $S_{3}$.

\footnotetext{
${ }^{57}$ Viewed as elements of $S_{3}=S_{4} /\left(\mathbb{Z}_{2} \times \mathbb{Z}_{2}\right)$ and working in the 'gauge' in which the fourth particle is fixed, the exchange elements that leave the three structures in (3.5) fixed respectively are (213), (321) and (132).

${ }^{58}$ See the discussion under (2.36) for a definition and listing of these cyclic elements.

${ }^{59}$ Restated, s, v, t are denoted by a vertex with valency $0,1,2$ respectively. The contraction of indices means connecting pairs of vertices by lines such that all the valencies are saturated.
} 

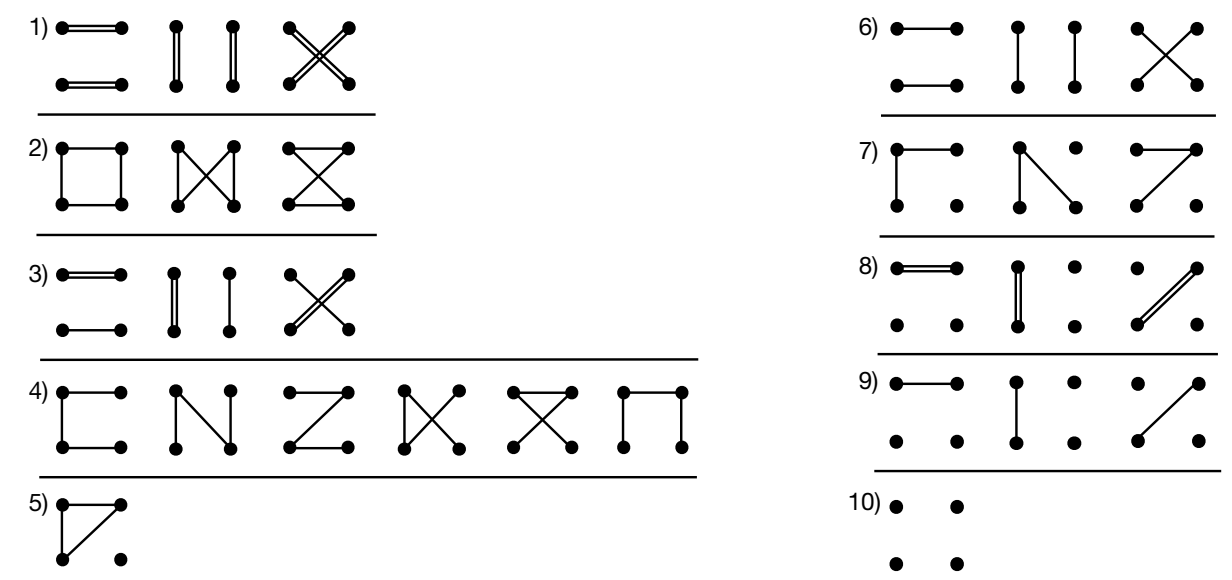

Figure 2. Parity even index structures of 4-graviton scattering.

Finally the single structure in the $\left.\mathbf{s}^{\otimes 4}\right|_{\mathbb{Z}_{2} \times \mathbb{Z}_{2}}$ is denoted by the very simple figure in the third line of figure 1 . The corresponding expression is simply $\alpha_{1} \alpha_{2} \alpha_{3} \alpha_{4}$, and clearly transforms in the $\mathbf{1}_{\mathbf{S}}$ representation of $S_{3}$. We denote it as $e_{\mathbf{S}}$. Using (2.34) it follows that the seven parity even photon structures for $D \geq 7$ transform under $S_{3}$ as

$$
7=3 \cdot \mathbf{1}_{\mathrm{S}}+2 \cdot \mathbf{2}_{\mathrm{M}}
$$

We now turn to the explicit construction of the 29 parity even structures index structures for gravitational scattering in $D \geq 7$ (see table 3 ). The structures in question are all listed in figure 2. Several comments are in order. First, we re-emphasize that individual diagrams in figure 2 are not always $\mathbb{Z}_{2} \times \mathbb{Z}_{2}$ invariant but by the corresponding figure, we mean the object obtained after $\mathbb{Z}_{2} \times \mathbb{Z}_{2}$ symmetrization. ${ }^{60}$ Second, we have arranged the graphs in figure 2 so that structures that lie in the same $\mathbb{Z}_{3}$ orbits all appear on the same line. More specifically, expressions denoted by the diagrams in lines 1), 2), 3), 6), 7), 8) and 9) all transform in the $\mathbf{3}$ representation of $S_{3}$. Terms signified by the diagrams in line 4) transform in the $\boldsymbol{6}_{\text {left }}$ dimensional representation (see around (2.36)). And the terms denoted by the diagrams in lines 5) and 10) transform in the trivial $\mathbf{1}_{\mathbf{S}}$ representation of $S_{3}$. Using (2.34), (2.35) it follows that the $S_{3}$ representation content of parity invariant graviton index structures is given by

$$
29=10 \cdot \mathbf{1}_{\mathbf{S}}+9 \cdot \mathbf{2}_{\mathbf{M}}+1 \cdot \mathbf{1}_{\mathbf{A}} .
$$

We now turn to a discussion of parity odd S-matrices in $D \geq 8$. Such S-matrices are linear in $\widetilde{\varepsilon}^{D-3}$ (see (2.19) for a definition). For $D \geq 8$ the number of free indices of $\widetilde{\varepsilon}^{D-3}$ (or $N(\widetilde{\varepsilon})^{D-3}$ ) tensor is $\geq 5$. As the only vectors available to contract with this tensor are the $4 \epsilon_{i}^{\perp}$, there are non parity odd S-matrix structures in $\geq 8$.

\footnotetext{
${ }^{60}$ In other words $\mathbb{Z}_{2} \times \mathbb{Z}_{2}$ images, i.e. images under double transpositions, are implicitly added in our diagrams when needed.
} 


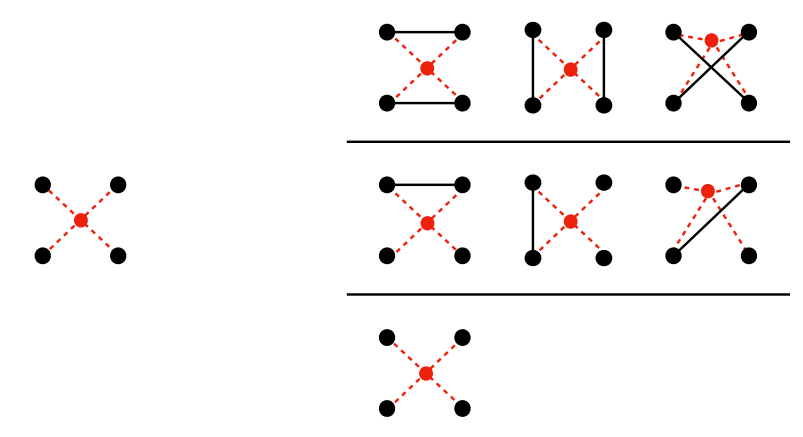

Figure 3. Parity odd index structures of 4-photon and 4-graviton scattering in $D=7$ respectively. The $\widetilde{\varepsilon}$ symbol is denoted by a red dot with $D-3$ valency.

Let us now construct the parity odd structures for photon S-matrix in $D=7$. The tensor $\widetilde{\varepsilon}^{4}$ has 4 free indices so it can be contracted with the $4 \epsilon_{i}^{\perp}$ in a unique way.

$$
o_{\mathbf{S}}^{D=7}=\widetilde{\varepsilon}_{\mu \nu \rho \sigma}^{4} \epsilon_{1}^{\perp \mu} \epsilon_{2}^{\perp \nu} \epsilon_{3}^{\perp} \rho \epsilon_{4}^{\perp} \sigma=\varepsilon_{\alpha \beta \gamma \mu \nu \rho \sigma} p_{1}^{\alpha} p_{2}^{\beta} p_{3}^{\gamma} \epsilon_{1}^{\perp \mu} \epsilon_{2}^{\perp \nu} \epsilon_{3}^{\perp} \rho \epsilon_{4}^{\perp} \sigma .
$$

Consequently there is a single parity odd structure in $\left.\rho^{\otimes 4}\right|_{\mathbb{Z}_{2} \times \mathbb{Z}_{2}}$ for the case of photons in seven dimensions. This S-matrix transforms in the $\mathbf{1}_{S}$ representation of $S_{3} \cdot{ }^{61}$ In equations

$$
1=1 \cdot \mathbf{1}_{\mathbf{S}}
$$

As for the case of parity even structures, it is useful to develop a graphical notation to denote parity odd contraction structures. Our graphs now have 5 vertices; four for each of the scattering particles and a red dot for $\widetilde{\varepsilon}^{D-3}$. The red vertex always has valency $D-3$ i.e. always has $D-3$ dotted lines emerging out of it. A dotted line between the red dot and, say, the vertex 1 denotes that $\epsilon_{1}^{\perp}$ has contracted with one of the free indices of $\widetilde{\varepsilon}^{D-3}$. The meaning of lines between usual vertices is the same as for the parity even diagrams. With these conventions in place, the unique parity odd structure for 4 photon scattering in $D=7$ is denoted by the first diagram in figure 3 .

There are 7 parity odd structures for 4 graviton scattering in $D=7$. These structures are depicted in the second part of figure 3 . The first two lines of this part of the figure depict structures that transform under $S_{3}$ in the $\mathbf{3}$ representation. The single structure in the last line, of course, transforms in the $\mathbf{1}_{S}$ representation. In equations, the 7 parity odd graviton scattering structures in $D=7$ transform as

$$
7=3 \cdot \mathbf{1}_{\mathrm{S}}+2 \cdot \mathbf{2}_{\mathbf{M}}
$$

In appendix D we have presented a detailed construction of all basis index structures - and their $S_{3}$ transformation properties for $D=6,5,4,3$. Here we content ourselves with

\footnotetext{
${ }^{61}$ In order to obtain the correct symmetry transformation property of this term it is important to permute the momenta that go into the definition of $\widetilde{\varepsilon}^{4}$ along with the factors of $\epsilon_{i}^{\perp}$. In order to avoid errors it is best to express $\widetilde{\varepsilon}^{4}$ in term of $\varepsilon$ using (2.19) - as has been done on the r.h.s. of (3.9) - before performing permutations.
} 


\begin{tabular}{|c|c|c|c|c|c|c|}
\hline photons & \multicolumn{3}{|c|}{ even } & \multicolumn{3}{c|}{ odd } \\
\hline & $n_{\mathbf{1}_{\mathbf{S}}}$ & $n_{\mathbf{2}_{\mathbf{M}}}$ & $n_{\mathbf{1}_{\mathbf{A}}}$ & $n_{\mathbf{1}_{\mathbf{S}}}$ & $n_{\mathbf{2}_{\mathbf{M}}}$ & $n_{\mathbf{1}_{\mathbf{A}}}$ \\
\hline$D \geq 8$ & 3 & 2 & 0 & 0 & 0 & 0 \\
\hline$D=7$ & 3 & 2 & 0 & 1 & 0 & 0 \\
\hline$D=6$ & 3 & 2 & 0 & 0 & 0 & 1 \\
\hline$D=5$ & 3 & 2 & 0 & 0 & 0 & 0 \\
\hline$D=4$ & 3 & 1 & 0 & 2 & 0 & 0 \\
\hline$D=3$ & 1 & 0 & 0 & 0 & 0 & 1 \\
\hline gravitons & \multicolumn{3}{|c|}{ even } & & odd \\
\hline & $n_{\mathbf{1}_{\mathbf{S}}}$ & $n_{\mathbf{2}_{\mathbf{M}}}$ & $n_{\mathbf{1}_{\mathbf{A}}}$ & $n_{\mathbf{1}_{\mathbf{S}}}$ & $n_{\mathbf{2}_{\mathbf{M}}}$ & $n_{\mathbf{1}_{\mathbf{A}}}$ \\
\hline$D \geq 8$ & 10 & 9 & 1 & 0 & 0 & 0 \\
\hline$D=7$ & 10 & 9 & 1 & 3 & 2 & 0 \\
\hline$D=6$ & 9 & 9 & 1 & 0 & 3 & 3 \\
\hline$D=5$ & 7 & 7 & 1 & 0 & 1 & 1 \\
\hline$D=4$ & 3 & 1 & 0 & 2 & 0 & 0 \\
\hline$D=3$ & - & - & - & - & - & - \\
\hline
\end{tabular}

Table 4. Number of parity even and parity odd index structures for 4-photon and 4-graviton S-matrix as various dimensions.

merely tabulating the $S_{3}$ transformation properties of the basis of the bare module in every dimension in table 4 . Note of course that, for photons and gravitons, both for parity even and odd, in any given dimension, $n_{\mathbf{1}_{\mathrm{S}}}+2 n_{\mathbf{2}_{\mathrm{M}}}+n_{\mathbf{1}_{\mathbf{A}}}$ equals the total number of structures tabulated in 3

\section{Counting local Lagrangians}

\subsection{Local Lagrangians and local S-matrices}

As we have mentioned in section 2.4, one of the principal goals in this paper is the enumeration and explicit construction all local or polynomial S-matrices (recall Lorentz and gauge invariant S-matrix are said to be local, analytic or polynomial if they are polynomial function of the variables $\left.\left(p_{i}, \epsilon_{i}\right)\right)$. The set of local four particle S-matrices is, of course, closely related to the set of all local quartic Lagrangians. ${ }^{62}$ There is an obvious map from the set of local gauge invariant quartic vertices to the set of local 4 particle S-matrices. This

\footnotetext{
${ }^{62} L(x)$ is a local Lagrangian if it is a function only of fields and their derivatives evaluated at $x$, subject to the restriction that the number of derivatives acting on any field is bounded from above (i.e. is finite).
} 
map, however, is many to one. Two Lagrangians generate the same S-matrix if they differ only by total derivatives when evaluated on-shell (we will make this statement completely precise below). The map from equivalence classes of Lagrangians to S-matrices can also be inverted. Given polynomial S-matrix one can construct a local quartic Lagrangian vertex that is invariant under linearized gauge transformations that gives rise to that S-matrix. ${ }^{63}$ There exists, in other words, a one to one map from the space of local equivalence classes of Lagrangians and local S-matrices; the classification of local S-matrices is the same as the classification of equivalence classes of local Lagrangians.

In the rest of this subsection we pause to explore the space of inequivalent Lagrangians - and their connection with inequivalent four particle scattering - more carefully, separately for the case of scalars, photons and gravitons.

\subsubsection{Scalars}

In this subsubsection we closely follow the analysis of [21]. Consider a theory of real massless scalars $\phi$ invariant under the $\mathbb{Z}_{2}$ transformations $\phi \rightarrow-\phi$. We wish to study the most general local action for this theory, retaining only those terms that affect four scalar scattering. $\mathbb{Z}_{2}$ symmetry ensures that we need only consider terms in the Lagrangian that are quadratic and quartic. We require that our scalar propagator have a single massless pole at $p^{2}=0$. This constraint (plus a convenient choice of normalization) determines the quadratic term in the Lagrangian to be

$$
S_{2}=-\frac{1}{2} \int d^{D} x \partial_{\mu} \phi \partial^{\mu} \phi
$$

The most general local quartic interaction Lagrangian takes the form

$$
S_{4}=\int d^{D} x L_{4}, \quad L_{4}=\sum a_{m_{1}, m_{2}, m_{3}, m_{4}} \partial^{m_{1}} \phi \partial^{m_{2}} \phi \partial^{m_{3}} \phi \partial^{m_{4}} \phi
$$

where the schematic summation in the last line of (4.2) runs over both the number of derivatives $m_{i}$ on the fields $\phi$ as well as the distinct ways of contracting the various derivative indices. A tree diagram computation using the action (4.2) yields a 4 scalar S-matrix. This procedure establishes a map from the space of local Lagrangians $L_{4}(4.2)$ and the set of local S-matrices. As mentioned in the introduction to this subsection, however, this map is many to one. Two Lagrangians $L_{4}$ yield the same S-matrix if

- They differ by a total derivative.

- They can be related to each other by a field redefinition.

Consider a field redefinition of the schematic form

$$
\begin{aligned}
\phi & \rightarrow \phi+\delta \phi \\
\delta \phi & =\left(\sum b_{m_{1}, m_{2}, m_{3}} \partial^{m_{1}} \phi \partial^{m_{2}} \phi \partial^{m_{3}} \phi\right)
\end{aligned}
$$

\footnotetext{
${ }^{63}$ The map from S-matrices to Lagrangians played an important role in [21] for scalars.
} 
Up to terms of sextic and higher order that we ignore, the field redefinition (4.3) shifts $L_{4}$ by

$$
\delta L_{4}=\partial^{2} \phi\left(\sum b_{m_{1}, m_{2}, m_{3}} \partial^{m_{1}} \phi \partial^{m_{2}} \phi \partial^{m_{3}} \phi\right)
$$

It follows that the space of quartic terms $L_{4}$ may be divided up into equivalence classes. Two local quartic terms lie in the same equivalence class either if they agree up to a total derivative when we set $\partial^{2} \phi=0 .{ }^{64}$

The map between equivalence classes of $L_{4}$ and four scalar S-matrices is one to one. To see this it is useful to move to momentum space. Let

$$
\begin{aligned}
\phi(x) & =\int \frac{d^{d} k}{(2 \pi)^{d}} e^{i k \cdot x} \widetilde{\phi}(k) \\
L_{4} & =\int \prod_{i} \frac{d^{d} k}{(2 \pi)^{d}} e^{i\left(\sum_{j} k_{j} x_{j}\right)} \widetilde{L}_{4}\left(k_{1}, k_{2}, k_{3}, k_{4}\right) \widetilde{\phi}\left(k_{1}\right) \widetilde{\phi}\left(k_{2}\right) \widetilde{\phi}\left(k_{3}\right) \widetilde{\phi}\left(k_{4}\right)
\end{aligned}
$$

It follows from the discussion above that $\widetilde{L}_{4}^{1}\left(k_{1}, k_{2}, k_{3}, k_{4}\right)$ and $\widetilde{L}_{4}^{2}\left(k_{1}, k_{2}, k_{3}, k_{4}\right)$ lie in the same equivalence class if and only if

$$
\begin{aligned}
\widetilde{L}_{4}^{1}\left(k_{1}, k_{2}, k_{3}, k_{4}\right) & =\widetilde{L}_{4}^{2}\left(k_{1}, k_{2}, k_{3}, k_{4}\right) \quad \text { when } \\
\sum_{i=1}^{4} k_{i} & =0, \quad \text { and } \quad k_{i}^{2}=0, i=1 \ldots 4
\end{aligned}
$$

But $\widetilde{L}_{4}\left(k_{1}, k_{2}, k_{3}, k_{4}\right)$, evaluated subject to (4.7), is precisely the tree level S-matrix evaluated using the Lagrangian $L .{ }^{65}$ It follows that the equivalence classes of quartic Lagrangian terms are in fact labelled by their tree level S-matrix. Moreover any polynomial S-matrix $S\left(k_{1}, k_{2}, k_{3}, k_{4}\right)$ (defined on the space of momenta (4.7)) can be extended to a polynomial function of unconstrained variables $k_{1}, k_{2}, k_{3}$ and $k_{4}$ in many inequivalent ways. Choose any such extension, name it $\widetilde{L}_{4}\left(k_{1}, k_{2}, k_{3}, k_{4}\right)$. The equation (4.5) then may be viewed as a map from polynomial S-matrices to (any particular representative of) the equivalence classes of local Lagrangians. It follows that local 4 scalar S-matrices are in one to one correspondence with the equivalence classes $L_{4}$ described in this subsubsection.

Note that a complete classification of scalar field primaries transforming in any representation and with arbitrary number of $\phi \mathrm{s}$ has been carried out using algebraic methods in $[29,30]$.

\subsubsection{Photons}

The discussion of electromagnetic Lagrangians parallels that of the previous subsubsection in many respects but also has some new elements. To ensure gauge invariance, in this subsection we study Lagrangians built only out of the field strengths $F_{\mu \nu}{ }^{66}$ and work order

\footnotetext{
${ }^{64}$ In the introduction to this subsection we mentioned that Lagrangians that are 'on-shell equivalent' generate the same S-matrices. In the context of the scalar theory we study in this subsubsection, the precise meaning of 'on-shell equivalent' is 'obeys the equation $\partial^{2} \phi=0$. In the case of the photon/graviton studied in subsequent sub subsections, 'on-shell equivalent' means obeys the (free Maxwell) / (vacuum Einstein) equations respectively.

${ }^{65} \mathrm{Up}$ to a universal normalization factor.

${ }^{66}$ This class covers almost all gauge invariant electromagnetic Lagrangians. The exceptions have to do with Chern Simons terms which we ignore in this subsection but which we will account for later in the paper.
} 
by order in powers of the field strength. We require our vector field in Lorentz gauge have a propagator proportional to $\eta_{\mu \nu} / p^{2}$. This condition together with a convenient choice of normalization determines the quadratic term in our Lagrangian to be

$$
S_{2}=-\frac{1}{4} \int d^{D} x F_{\mu \nu} F^{\mu \nu} .
$$

As we do not demand that our theory has a $\mathbb{Z}_{2}$ invariance, the Lagrangian could have cubic terms in the field strength. In appendix E, however, we demonstrate that it is always possible to perform a field redefinition of the form

$$
\begin{aligned}
A_{\mu} & \rightarrow A_{\mu}+\delta A_{\mu} \\
\delta A_{\mu} & =\left(\sum d_{n_{1}, n_{2}} \partial^{m_{1}} F \partial^{m_{2}} F\right)_{\mu}
\end{aligned}
$$

to set the cubic action $S_{3}$ to zero.

It follows that the 3 photon S-matrix vanishes. This also means that the most general classical S-matrix of a theory of photons (and no other fields) has no exchange poles and so is purely local in nature, as was the case of the $\mathbb{Z}_{2}$ invariant scalar theory of the previous subsubsection.

The most general quartic Lagrangian takes the schematic form

$$
S_{4}=\int d^{D} x L_{4}, \quad L_{4}=\sum a_{m_{1}, m_{2}, m_{3}, m_{4}} \partial^{m_{1}} F \partial^{m_{2}} F \partial^{m_{3}} F \partial^{m_{4}} F
$$

(where all indices on $\partial_{\mu}$ and $F_{\mu \nu}$ have been suppressed). A field redefinition of the schematic form

$$
\begin{aligned}
A_{\mu} & \rightarrow A_{\mu}+\delta A_{\mu} \\
\delta A_{\mu} & =\left(\sum b_{n_{1}, n_{2} n_{3}} \partial^{m_{1}} F \partial^{m_{2}} F \partial^{m_{3}} F\right)_{\mu}
\end{aligned}
$$

generates of shift of $L_{4}$

$$
\delta L_{4}=\partial_{\nu} F^{\mu \nu}\left(\sum b_{m_{1}, m_{2}, m_{3}} \partial^{m_{1}} F \partial^{m_{2}} F \partial^{m_{3}} F\right)_{\mu} .
$$

It follows that Lagrangians $L_{4}$ that differ from each other by

- Total derivatives

- Terms that include $\partial_{\nu} F^{\nu \mu}$

- Terms that include $\partial_{[\alpha} F_{\beta \gamma]}$ as a factor (such terms are, of course, identically zero when expressed in terms of $A_{\mu}$ )

generate the same S-matrix and should be treated as equivalent to each other.

As in the case of scalars, equivalence classes of Lagrangians are labelled by their Smatrices. Moreover we expect that there is a one to one map from classes of local Lagrangians to polynomial S-matrices. See appendix E.2 for a discussion. 


\subsubsection{Gravitons}

In order to ensure diffeomorphism invariance, in this section we study gravitational Lagrangians constructed out of the Riemann tensor ${ }^{67}$ and work order by order in powers of the Riemann tensor. ${ }^{68}$ Before commencing our discussion we pause to define some terminology. Throughout this subsubsection the symbol $H_{\mu \nu}^{(n)}\left[R_{\alpha \beta \gamma \delta}\right]$ will denote the most general local functional that is rank 2 symmetric tensor and that is $n^{\text {th }}$ order in the Riemann tensor, but with arbitrary powers of the metric and arbitrary numbers of symmetrized derivatives. ${ }^{69}$

The unique diffeomorphism invariant action that is linear in Riemann tensors is, of course, the Einstein action

$$
S_{E}=\int \sqrt{-g} R \text {. }
$$

In appendix E we demonstrate that the field redefinition

$$
\delta g_{\mu \nu}=H_{\mu \nu}^{(1)}\left[R_{\alpha \beta \gamma \delta}\right]
$$

may be used to cast the most general Lagrangian, quadratic in Riemann tensors, into the form

$$
S=S_{E}+S_{G B}+\int \mathcal{O}\left(R_{\alpha \beta \gamma \delta}\right)^{3}
$$

where,

$$
\begin{aligned}
S_{G B} & =\int \sqrt{-g} \delta_{[a}^{g} \delta_{b}^{h} \delta_{c}^{i} \delta_{d]}^{j} R_{a b}{ }^{g h} R_{c d}{ }^{i j} \\
& \propto \int \sqrt{-g}\left(R^{2}-4 R^{\mu \nu} R_{\mu \nu}+R^{\mu \nu \rho \sigma} R_{\mu \nu \rho \sigma}\right) .
\end{aligned}
$$

and $\mathcal{O}\left(R_{\alpha \beta \gamma \delta}\right)^{3}$ denotes all terms that are of cubic or higher order in the Riemann tensor. ${ }^{70}$ In other words Einstein-Gauss-Bonnet is the most general action quadratic in the Riemann tensor up to total derivatives or terms that involve explicit factors of $R_{\mu \nu}$ and the Ricci scalar $R .^{71}$

\footnotetext{
${ }^{67}$ This covers almost all diffeomorphism invariant gravity Lagrangians. The exceptions to this rule are gravitational Chern Simons terms which we ignore in this subsubsection, but whose effects we account for later in this paper.

${ }^{68}$ We define an action to be of $m^{\text {th }}$ order in Riemann tensors if there is no manipulation that allows us to express the same action as an expression of higher orders in Riemann tensors in a local manner. For instance, we count an expression containing $\left[\nabla_{\mu}, \nabla_{\nu}\right]$ acting on $m$ explicit copies of the Riemann tensor as being of degree $m+1$ as the antisymmetric combination of derivatives can be replaced by a Riemann tensor. An expression that is of $m^{\text {th }}$ order in Riemann tensors does not contribute to $n$ point scattering amplitudes of gravitons for $n<m$. Terms of $m^{\text {th }}$ order typically do contribute to S-matrices for $m$ and higher point S-matrices. There are exceptions to this last rule; it is sometimes possible for an object to be of $m^{\text {th }}$ order in Riemann tensors but to contribute to S-matrices only at order $m+1$ or higher.

${ }^{69}$ One example of an allowed functional is

$$
H_{\mu \nu}^{(1)}=a R g_{\mu \nu}+\nabla^{2} R g_{\mu \nu}+c R_{\mu \nu}+d \nabla^{\alpha} \nabla \beta R_{\alpha \mu \beta \nu} \ldots
$$

${ }^{70}$ In four dimensions the Gauss-Bonnet term vanishes identically.

${ }^{71}$ In particular the Einstein equations $R_{\mu \nu}=0$ tell us that we need only work with Riemann tensor terms containing Ricci tensor or Ricci scalar are effectively trivial.
} 
When evaluated in a spacetime of the form

$$
g_{\mu \nu}=\eta_{\mu \nu}+h_{\mu \nu}
$$

it turns out that the Gauss-Bonnet term in (4.15) starts out at order $h^{3}$ (up to total derivatives). It follows, in other words, that - despite the appearance - the GaussBonnet term does not modify the Einstein propagator but does contribute to three point scattering of gravitons. This term is, of course, topological in $D=4$.

In appendix E we next demonstrate that field redefinitions of the form

$$
\delta g_{\mu \nu}=H_{\mu \nu}^{(2)}\left[R_{\alpha \beta \gamma \delta}\right]
$$

can be used to cast the most general cubic correction to the Einstein-Gauss-Bonnet action into the form

$$
\begin{aligned}
S= & S_{E}+S_{G B}+a S_{R^{3}}^{(1)}+b \chi_{6}+\int \sqrt{-g}\left(\mathcal{O}\left(R_{\alpha \beta \gamma \delta}\right)^{4}\right) \\
S_{R^{3}}^{(1)}= & \int \sqrt{-g}\left(R^{p q r s} R_{p q}{ }^{t u} R_{r s t u}+2 R^{p q r s} R_{p r}{ }^{t}{ }^{u} R_{q t s u}\right) \\
\chi_{6}= & \int \sqrt{-g}\left(\frac{1}{8} \delta_{[a}^{g} \delta_{b}^{h} \delta_{c}^{i} \delta_{d}^{j} \delta_{e}^{k} \delta_{f]}^{l} R_{a b}{ }^{g h} R_{c d}{ }^{i j} R_{e f}{ }^{k l}\right) \\
= & \int \sqrt{-g}\left(4 R_{a b}{ }^{c d} R_{c d}{ }^{e f} R_{e f}{ }^{a b}-8 R_{a b}^{c d} R_{c d}^{e f} R_{e f}{ }^{a b}-24 R_{a b c d} R^{a b c}{ }_{e} R^{d e}+3 R_{a b c d} R^{a b c d} R\right. \\
& \left.\quad+24 R_{a b c d} R^{a c} R^{b d}+16 R_{a}^{b} R_{b}{ }^{c} R_{c}{ }^{a}-12 R_{a}^{b} R_{b}{ }^{a} R+R^{3}\right)
\end{aligned}
$$

In other words, Einstein-Gauss-Bonnet corrected by two specific cubic terms is the most general action cubic in Riemann tensors - up to total derivatives and terms that vanish by the Einstein equations. ${ }^{72}$

When evaluated on the metric (4.17), the term $\chi_{6}$ starts out at order $h_{\mu \nu}^{4}$ (up to total derivatives). It follows in particular that this term does not contribute to three graviton scattering. $\chi_{6}$ is field redefinition equivalent to the simpler looking expression

$$
S_{R^{3}}^{(2)}=\int \sqrt{-g}\left(R^{p q r s} R_{p q}{ }^{t u} R_{r s t u}-2 R^{p q r s} R_{p r}{ }^{t}{ }^{u} R_{q t s u}\right)
$$

(obtained by setting all terms involving $R_{\mu \nu}$ in $\chi_{6}$ to zero). The reason that we prefer to use $\chi_{6}$ rather than (4.21) in our action is the following; when evaluated on the configuration (4.17), the expression $S_{R^{3}}^{(2)}$ is of order $h_{\mu \nu}^{4}$ only on-shell; when evaluated off-shell this expression is of order $h_{\mu \nu}^{3}$. As a consequence, while the actions $\chi_{6}$ and $S_{R^{3}}^{(2)}$ lead to the same polynomial graviton 4 point function, this scattering amplitude has its source purely in contact terms in the case of $\chi_{6}$, but in the more complicated sum of contact and exchange diagrams (which are polynomial as on-shell 3 point functions vanish) in the case of

\footnotetext{
${ }^{72}$ In four dimension another cubic term is present that is parity odd [31] $\varepsilon^{\alpha \beta \gamma \delta} R_{\alpha \beta a b} R_{\gamma \delta c d} R_{a b c d}$. This is dual to a parity violating three point function structure for stress tensors in three dimensions [32, 33]. We will not consider exchange due to this term in this paper.
} 
$S_{R^{3}}^{(2)}$. Consequently $\chi_{6}$ is clearly dynamically simpler than $S_{R^{3}}^{(2)}$, even though it superficially looks more complicated. $\chi_{6}$ also has other interesting properties; it vanishes identically in less than six dimensions, and is topological in $d=6$. In fact $\chi_{6}$ is sometimes called the ' 6 dimensional Euler density' [34, 35]. It is also the second in the sequence of Lovelock terms (the first is the Gauss-Bonnet term written above).

In contrast to $\chi_{6}, S_{R^{3}}^{(1)}$ does contribute to the three point functions. ${ }^{73}$ In fact the Einstein term, the Gauss-Bonnet term and $S_{R^{3}}^{(1)}$ each contribute to three graviton scattering. It follows that the most general 3 graviton S-matrix is a linear sum of 3 independent structures. The Einstein action is quadratic in derivatives and leads to a 3 graviton Smatrix proportional to

$$
\mathcal{A}^{R}=\left(\epsilon_{1} \cdot \epsilon_{2} \epsilon_{3} \cdot p_{1}+\epsilon_{1} \cdot \epsilon_{3} \epsilon_{2} \cdot p_{3}+\epsilon_{2} \cdot \epsilon_{3} \epsilon_{1} \cdot p_{2}\right)^{2}
$$

The Gauss-Bonnet action is quartic in derivatives and leads to a 3 graviton S-matrix proportional to ${ }^{74}$

$$
\mathcal{A}^{R^{2}}=\left(\epsilon_{1} \wedge \epsilon_{2} \wedge \epsilon_{3} \wedge p_{1} \wedge p_{2}\right)^{2}
$$

The Riemann cube term is sextic in derivatives and leads to a 3 graviton S-matrix proportional to

$$
\mathcal{A}^{R^{3}}=\left(\operatorname{Tr} F_{1} F_{2} F_{3}\right)^{2} .
$$

As the 3-graviton S-matrix is non vanishing, 4-graviton S-matrices that follow from the Lagrangian (4.19) have contributions from Feynman diagrams with a single graviton exchange. Such exchange diagrams lead to S-matrices that are not polynomial in $s, t$ and $u$ but instead have poles. We have explicitly evaluated the 4 graviton S-matrix that follows from the action (4.19). Our results are presented in section 7. Notice that the three graviton scattering amplitudes $\mathcal{A}^{R^{2}}$ and $\mathcal{A}^{R^{3}}$ are gauge invariant ${ }^{75}$ even off-shell (i.e. even if $k_{i}^{2} \neq 0$ ). It follows that exchange diagrams that sew together two of these vertices are individually gauge invariant. ${ }^{76}$ On the other hand $\mathcal{A}^{R}$ is not off-shell gauge invariant (though it certainly is on-shell gauge invariant). The sum of all channels of exchange diagrams involving either one or two copies of $\mathcal{A}^{R}$ is, therefore, gauge invariant only once we add the contribution of the relevant polynomial contact term (this is the explicit $4 h_{\mu \nu}$ term in the Gauss-Bonnet action or in $S_{R^{3}}^{(1)}$ in the case of an Einstein Gauss-Bonnet or $S_{R^{3}}^{(1)}$ exchange diagram or the explicit $4 h_{\mu \nu}$ term in the Einstein action in the case of an Einstein - Einstein exchange diagram.)

We pause here to note that the discussion of the previous paragraph illustrates the interesting interplay between the on-shell invariance of S-matrices under linearized gauge

\footnotetext{
${ }^{73}$ The specific choice we have made for $S_{R^{3}}^{(1)}$ is a bit arbitrary; we could have added any multiple of $S_{R^{3}}^{(2)}$ to it without changing its essential features.

${ }^{74}$ As remarked in the earlier footnote, this structure vanishes in $D=4$ but a parity odd structure appears in its place.

${ }^{75}$ I.e. are invariant under the shift $\epsilon_{i} \rightarrow \epsilon_{i}+k_{i}$.

${ }^{76} \mathrm{Off}$ shell gauge invariance of the three point function is relevant here because the intermediate graviton is off-shell in an exchange diagram.
} 
transformations and the non-linear off-shell gauge invariance of the Lagrangians that generate them. It might at first seem that the requirement of linearized gauge invariance of S-matrices is a weaker condition than the off-shell non-linear gauge invariance of Lagrangians; this is not the case. Consider, for example, four point scattering in the Einstein gravity (the Einstein-Einstein case discussed in the previous paragraph). As we have mentioned above, the exchange diagrams by themselves are not linearized-gauge invariant, but the four graviton contact structure in the Einstein-Hilbert Lagrangian is such that its linear-gauge transformations compensates the linear-gauge transformation of the exchange term to render the full S-matrix linear-gauge invariant. This works precisely because the non-linear gauge invariance of the Einstein Hilbert term relates cubic and quartic vertices (the gauge transformation of the cubic vertex linear in $h_{\mu \nu}$ cancels the linear gauge transformation of the quartic vertex i.e. gauge transformation that is independent of $h_{\mu \nu}$ ). This relation between the vertices guarantees that the sum of exchange and contact diagrams generates a 4 graviton S-matrix that is on-shell, linearized gauge invariant.

As we have mentioned above, $\chi_{6}$ does not contribute to 3 graviton scattering, but does contribute (polynomiallally) to four graviton scattering. The four graviton S-matrix that follows from this term is proportional to

$$
\begin{aligned}
T_{1} & =\left(\epsilon_{1} \wedge \epsilon_{2} \wedge \epsilon_{3} \wedge \epsilon_{4} \wedge k_{1} \wedge k_{2} \wedge k_{3}\right)^{2} \\
& \propto \delta_{[a}^{i} \delta_{s}^{j} \delta_{d}^{k} \delta_{f}^{l} \delta_{g}^{m} \delta_{h}^{n} \delta_{j]}^{p} \epsilon_{i}^{1} \epsilon_{j}^{2} \epsilon_{k}^{3} \epsilon_{l}^{4} p_{m}^{1} p_{n}^{2} p_{p}^{3} \epsilon^{1 a} \epsilon^{2 s} \epsilon^{3 d} \epsilon^{4 f} p^{1 g} p^{2 h} p^{3 j}
\end{aligned}
$$

Finally we turn to local Lagrangians of quartic or higher order in Riemann tensors. These terms, of course, do not contribute to 3 graviton scattering, but all give rise four graviton S-matrices that are polynomial in $\epsilon_{i}$ and $k_{i}$. As above field redefinitions of the form

$$
\delta g_{\mu \nu}=H_{\mu \nu}^{(3)}\left[R_{\alpha \beta \gamma \delta}\right]
$$

can be used to simplify the most general quartic correction to the Einstein-GaussBonnet-Riemann-cube action. Even up to the simplification afforded by the field redefinitions (4.26), however, the most general action that is quartic in Riemann tensors, turns out to be characterized by an infinite (rather than a finite, as was the case at quadratic and cubic order) number of parameters. In subsection (4.2) below we turn to the problem of enumerating such Lagrangians.

As in the previous subsubsection there is a close connection between gauge invariant local S-matrices and equivalence classes of quartic local Lagrangians built out of the Riemann tensor. To first approximation the relationship between these two structures is as for the gauge fields but there are some additional complications stemming from the non-linear nature of the gravitational field. The map from Lagrangians to S-matrices continues to be the obvious one. When evaluated on-shell, Lagrangians that differ only by total derivatives or terms of order $h^{5}$ or higher yield the same S-matrix. ${ }^{77}$ There is also a complication in the reverse map: it is possible for local S-matrices to correspond to Lagrangians that are of

\footnotetext{
${ }^{77}$ The stipulation about terms of higher order is necessary because the non-linearity of gravity makes it possible for two terms built out of four Riemann tensors, that are distinct even on-shell at the non-linear level, to agree at $\mathcal{O}\left(h^{4}\right)$.
} 
lower than quadratic order in the Riemann tensor, as we have already seen in the example of the second Riemann cube term above. Later in this paper we will come to grips with all these complications in a quantitative manner.

\subsection{Plethystic program for scalars}

In the previous subsection we have argued that enumeration of four scalar S-matrices is isomorphic to the enumeration equivalence classes of local Lagrangians, quartic in $\phi$. In this subsection we explicitly count these equivalence classes graded by number of derivatives.

It is useful to define the single letter partition function i.e. partition function over all the operators that involve a single field, modulo the free equation of motion. The space of such operators for scalars is spanned by

$$
\partial_{\mu_{1}} \partial_{\mu_{2}} \ldots \partial_{\mu_{l}} \phi, \quad \text { for } \quad l=0, \ldots \quad \text { subjected to } \partial_{\mu} \partial^{\mu} \phi=0 .
$$

The single letter partition function is easily obtained; (see e.g. [36, 37]); it is given by

$$
\begin{array}{rlrl}
i_{\mathbf{s}}(x, y) & =\operatorname{Tr} x^{\Delta} y_{i}^{H_{i}}=\left(1-x^{2}\right) \mathrm{D}(x, y) . & \\
\mathrm{D}(x, y) & =\left(\prod_{i=1}^{D / 2}\left(1-x y_{i}\right)\left(1-x y_{i}^{-1}\right)\right)^{-1} & & \text { for D even } \\
& =\left((1-x) \prod_{i=1}^{\lfloor D / 2\rfloor}\left(1-x y_{i}\right)\left(1-x y_{i}^{-1}\right)\right)^{-1} & & \text { for D odd. }
\end{array}
$$

Here $H_{i}$ stands for the Cartan elements of $\mathrm{SO}(D)$. The denominator factor $\mathrm{D}(x, y)$ encodes the tower of derivatives on $\phi(x)$ keeping track of the degree and the charges under the Cartan subgroup of $\mathrm{SO}(D)$. We have kept track of the Cartans of $\mathrm{SO}(D)$ because we will eventually need to project polynomials built out of scalar letters to the space of $\operatorname{SO}(D)$ singlets below.

Equivalence classes of scalar Lagrangians are given by scalar quartic polynomials of the expressions (4.27) modulo polynomials that are total derivatives. We will first enumerate all quartic scalar polynomials we can make out of (4.27) and then subtract those polynomials that are total derivatives. The partition function of polynomials of the expressions (4.27) - the so called multi-letter partition function is given by the formula of Bose statistics

$$
\sum_{k=1}^{\infty} t^{k} i_{\mathbf{s}}^{(k)}(x, y)=\exp \left(\sum_{n=1}^{\infty} \frac{t^{n}}{n} i_{\mathbf{s}}\left(x^{n}, y^{n}\right)\right) .
$$

where $i_{\mathrm{s}}^{(k)}$ is the partition function over $k$-letter partition function, $i_{\mathrm{s}}^{(1)}=i_{\mathrm{s}}$.

The four-letter partition function — relevant for counting quartic Lagrangians — is easily read off from equation (4.29):

$i_{\mathbf{s}}^{(4)}(x, y)=\frac{1}{24}\left(i_{\mathbf{s}}^{4}(x, y)+6 i_{\mathbf{s}}^{2}(x, y) i_{\mathbf{s}}\left(x^{2}, y^{2}\right)+3 i_{\mathbf{s}}^{2}\left(x^{2}, y^{2}\right)+8 i_{\mathbf{s}}(x, y) i_{\mathbf{s}}\left(x^{3}, y^{3}\right)+6 i_{\mathbf{s}}\left(x^{4}, y^{4}\right)\right)$.

This partition function over four particle states includes operators that are total derivatives which we wish to remove. In conformal field theory language, this means we want to 
count only primary scalar quartic operators. Assuming that there are no null states in the multiplet, if the character of the conformal primary is $P(x, y)$ then the character over its entire multiplet is given by $P(x, y) \mathrm{D}(x, y)$ where $\mathrm{D}(x, y)$ encodes the contribution coming from the tower of derivatives. So in order to obtain only the partition function over primaries, we need to divide $i_{\mathbf{s}}^{(4)}(x, y)$ by $\mathrm{D}(x, y) .{ }^{78}$ The partition function over polynomials of (4.27), modulo total derivatives is given by

$$
i_{\mathbf{s}}^{(4)}(x, y) / \mathrm{D}(x, y) .
$$

The partition function over scalar operators is now obtained simply by projecting onto $\mathrm{SO}(D)$ invariant states. This is achieved by integrating $i_{\mathrm{s}}^{(4)}(x, y) / \mathrm{D}(x, y)$ over the Haar measure of the group. The resulting integral can be gauge fixed to the Cartan subgroup with the measure that is the $\mathrm{SO}(D)$ version of the Van der Monde determinant $\Delta\left(y_{i}\right)$ (see appendix $\mathrm{H}$ for details)

$$
I_{\mathbf{s}}^{D}(x):=\oint \prod_{i=1}^{\lfloor D / 2\rfloor} d y_{i} \Delta\left(y_{i}\right) i_{\mathbf{s}}^{(4)}(x, y) / \mathrm{D}(x, y) .
$$

This integral and its generalizations to higher points were used to enumerate $n$-point scalar S-matrices in $D=3$ and $D=4$ in [11].

It is somewhat cumbersome to evaluate this integral analytically for general dimensions. However it is easy to come up with a conjecture for the final answer. Parity invariant scalar scattering amplitudes are the same in every $D \geq 3$. The argument is similar to the one made for photons and gravitons (about asymptotic dimensions being 7 ) in the previous section. For $D \geq 3$, the entire 4-particle scalar particle scattering can be chosen to lie in a 3-plane and the remaining dimensions are mere spectators (unlike photons and gravitons, there are no polarizations that can occupy transverse dimensions). Moreover there are no parity odd S-matrices for $D \geq 4$ while such structures do exist for $D=3 .{ }^{79}$ The correspondence between equivalence classes of Lagrangians and S-matrices leads us to conjecture that the result of the integral (4.32) is independent of $D$ for $D \geq 4$. We have obtained some direct evidence for this conjecture by using Mathematica to evaluate (4.32) in a power series in $x$ up to $\mathcal{O}\left(x^{20}\right)$ in $D=4,5,6,7$, and verifying the results are independent of $D$ (and in fact equal to the results of the large $D$ computation, see below).

Given the conjecture described at the end of the previous paragraph, it is easy to evaluate (4.32) for all $D \geq 4$. This is done analytically by evaluating the integral in (4.32) in the large $D$ limit. The details of the computation are presented in appendix H. Our final results - together with the results in $D=3,2$ (in these special cases the integral is easily separately evaluated analytically) are tabulated results in 5 (with $\mathrm{D} \equiv 1 /\left(\left(1-x^{4}\right)\left(1-x^{6}\right)\right)$ ).

\footnotetext{
${ }^{78}$ As we will explain later, the assumption of not having null states is always valid for the scalar case but fails in $D=9$ both for photon and gravitons. In that case, we need to take care of the null state separately.

${ }^{79}$ The reason for this is simple. A parity odd S-matrix is proportional to $\epsilon_{\mu_{1} \ldots \mu_{D}}$ All indices of this tensor have to contract with some vector. However there are only three independent vectors in 3 dimensional scattering; namely $k_{1}, k_{2}$ and $k_{3}$. It follows that parity odd S-matrices exist in $D=3$ but not for $D \geq 4$.
} 


\begin{tabular}{|l|l|}
\hline dimension & scalar partition function \\
\hline$D \geq 4$ & D \\
\hline$D=3$ & $\left(1+x^{9}\right) \mathrm{D}$ \\
\hline$D=2$ & $\left(1-x^{6}\right) \mathrm{D}$ \\
\hline
\end{tabular}

Table 5. Partition function over the space of Lagrangians involving four $\phi$ 's. This includes both parity even and parity odd Lagrangians. $\mathrm{D} \equiv 1 /\left(\left(1-x^{4}\right)\left(1-x^{6}\right)\right)$.

Note in particular that $I_{\mathrm{s}}(x)$ agrees with the partition function over $S_{3}$ symmetric polynomials of $s, t, Z_{\mathbf{1}_{\mathbf{S}}}(x)$ for $D \geq 4$. This is entirely expected because the scalar Smatrices are precisely parameterized by $S_{3}$ symmetric polynomials of $s, t$. So this exercise serves as a check of something that we already know and expect. We will get new and more interesting results when we apply this machinery to Lagrangians of photons and gravitons.

\subsection{Photons and gravitons}

The single letter partition function for scalars $i_{\mathbf{s}}(x, y)$ can be thought of as the following sum

$$
i_{\mathrm{s}}(x, y)=1+x \chi_{\square}+x^{2} \chi_{\varpi}+x^{3} \chi_{\text {๓ }}+x^{4} \chi_{\text {巴 }}+x^{5} \chi_{\text {巴س }} \cdots
$$

Here $\chi_{R}\left(y_{i}\right)$ is the character of representation $R$ of $\mathrm{SO}(D)$. We have suppressed the arguments $y_{i}$ of $\chi_{R}$ in the above formula and will continue to do so when there is no danger of confusion. In the rest of the section, Young diagrams stand for $\mathrm{SO}(D)$ representations unless otherwise mentioned. That only symmetric traceless representations of $\mathrm{SO}(D)$ appear is clear from the explicit form of the descendants (4.27). These are also the representations of scalar spherical harmonics. This is also expected, because through state-operator map, the single-letter local operators are in one to one correspondence with single particle states on sphere $S^{D-1}$. The spectrum of states is precisely that of the scalar spherical harmonics.

For photons, the single letter partition function over gauge invariant operators is obtained by acting derivatives on the field strength $F_{\mu \nu}$, subjected to the equation of motion $\partial^{\mu} F_{\mu \nu}=0$ and the Bianchi identity $\partial_{[\rho} F_{\mu \nu]}=0$. The representations appearing after the action of a single derivative on $F_{\mu \nu}$ can be obtained by taking the direct product of rank 2 antisymmetric with a vector,

$$
\boxminus \otimes \square=\square \oplus \boxminus \oplus \square \text {. }
$$

The second and third representations stand for the Bianchi identity and equation of motion respectively, both of which vanish. So we get a single irreducible representation after the action of one derivative. Action of another derivative is obtained by the tensor product,

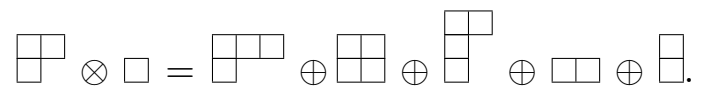

Again, the third and fourth representations vanish due to the Bianchi identity and equation of motion respectively. The last one vanishes because $\partial_{\mu} \partial^{\mu}=0$ (this follows from the 
Bianchi identity and the equation of motion), and the second vanishes because the two derivatives can not be anti-symmetrized. As a result, we again get the first one to be the only irreducible representation. This reasoning continues in general and we get

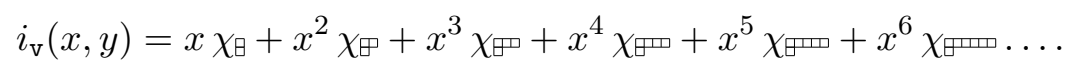

The first term is $F_{\mu \nu}$ and has a single derivative, hence a single power of $x$. These are precisely the representations of vector spherical harmonics, which are the states of a gauge invariant vector field on $S^{D-1}$. This series is summed in appendix G. The final result, which was also derived in e.g. [37], is given by

$$
i_{\mathrm{v}}(x, y)=\left(\left(\left(x-x^{3}\right) \chi_{\square}-\left(1-x^{4}\right)\right) \mathrm{D}(x, y)+1\right) / x .
$$

For gravitons, the single letter partition function over gauge invariant operators is obtained by acting derivatives on the Riemann tensor $R_{\mu \nu \alpha \beta}$, subjected to the equation of motion $\partial^{\mu} R_{\mu \nu \alpha \beta}=0$ and the Bianchi identity $\partial_{[\rho} R_{\mu \nu] \alpha \beta}=0$. Recall that the Riemann tensor enjoys the symmetries,

$$
R_{\mu \nu \alpha \beta}=R_{\alpha \beta \mu \nu}=-R_{\nu \mu \alpha \beta}=-R_{\mu \nu \beta \alpha}, \quad R_{\mu[\nu \alpha \beta]}=0 .
$$

It belongs to the following representation of $\mathrm{SO}(D)$,

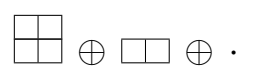

The irreducible representations correspond to the Weyl tensor, Ricci tensor and Ricci scalar respectively. The last two representations vanish on-shell and we are only left with the Weyl tensor. Note also that this representation is precisely the representation of the first tensor spherical harmonic. Just like the case of scalar and photon, we expect the graviton single letter partition function to be equal to the generating function for characters of tensor spherical harmonics.

$$
i_{\mathrm{t}}(x, y)=x^{2} \chi_{\boxplus}+x^{3} \chi_{\boxplus}+x^{4} \chi_{\boxplus}+x^{5} \chi_{\boxplus \amalg}+x^{6} \chi_{\boxplus m}+x^{7} \chi_{\boxplus m} \ldots
$$

The first term comes from $R_{\mu \nu \alpha \beta}$ and has a two derivatives, hence two powers of $x$. This series is summed in appendix G.

$$
i_{\mathrm{t}}(x, y)=\left(\left(\left(x^{2}-x^{4}\right)\left(1+\chi_{\varpi}\right)-\left(x-x^{5}\right) \chi_{\triangleright}\right) \mathrm{D}(x, y)+x^{2} \chi_{\boxminus}+x \chi_{\triangleright}\right) / x^{2} .
$$

Equipped with the single letter partition functions for photons (4.37) and gravitons (4.41), the partition function over quartic vertices is obtained in the same way as for scalars. We simply change the subscript $\mathbf{s}$ to $\mathrm{v}$ and $\mathrm{t}$ respectively in equation (4.29), (4.30) and (4.32). Again, this computation difficult to perform analytically in general dimensions. As argued earlier, we naively expect the counting of S-matrices to be independent of $D$ for $D \geq 7$. As we will discuss in detail below, however, there are subtleties concerning parity odd operators and null states for $D=7,8,9$. For this reason we conjecture that the photon and graviton versions of (4.27) are independent of $D$ for $D \geq 10$. Assuming this conjecture 


\begin{tabular}{|l|l|}
\hline dimension & photon partition function \\
\hline$d \geq 10$ & $x^{4}\left(2+3 x^{2}+2 x^{4}\right) \mathrm{D}$ \\
\hline$d=9$ & $x^{4}\left(2+3 x^{2}+2 x^{4}\right) \mathrm{D}-x^{5}$ \\
\hline$d=8$ & $x^{4}\left(2+3 x^{2}+2 x^{4}\right) \mathrm{D}+x^{4}$ \\
\hline$d=7$ & $x^{4}\left(x^{-1}+2+3 x^{2}+2 x^{4}\right) \mathrm{D}-x^{3}$ \\
\hline$d=6$ & $x^{4}\left(2+3 x^{2}+2 x^{4}+x^{8}\right) \mathrm{D}$ \\
\hline$d=5$ & $x^{4}\left(2+3 x^{2}+2 x^{4}\right) \mathrm{D}$ \\
\hline$d=4$ & $x^{4}\left(2+3 x^{2}+2 x^{4}-\left(x^{4}+x^{6}\right)+1+2 x^{2}+x^{4}-\left(x^{4}+x^{6}\right)\right) \mathrm{D}$ \\
\hline$d=3$ & $x^{4}\left(1+x^{2}+x^{4}-\left(x^{4}+x^{6}\right)+x^{5}\right) \mathrm{D}$ \\
\hline
\end{tabular}

Table 6. Partition function over the space of Lagrangians involving four $F_{\mu \nu}$ 's. This includes both parity even and parity odd Lagrangians. Recall $\mathrm{D} \equiv 1 /\left(\left(1-x^{4}\right)\left(1-x^{6}\right)\right)$. Blue: Parity Even. Red: Parity Odd. Yellow: Plethystic Miscount.

\begin{tabular}{|l|l|}
\hline dimension & graviton partition function \\
\hline$d \geq 10$ & $x^{8}\left(x^{-2}+6+9 x^{2}+10 x^{4}+3 x^{6}\right) \mathrm{D}-\left(x^{6}-x^{8}\right)$ \\
\hline$d=9$ & $x^{8}\left(x^{-2}+6+9 x^{2}+10 x^{4}+3 x^{6}\right) \mathrm{D}-\left(x^{6}-x^{8}\right)-2 x^{9}$ \\
\hline$d=8$ & $x^{8}\left(x^{-2}+6+9 x^{2}+10 x^{4}+3 x^{6}\right) \mathrm{D}-\left(x^{6}-x^{8}\right)+2 x^{8}$ \\
\hline$d=7$ & $x^{8}\left(x^{-2}+6+9 x^{2}+10 x^{4}+3 x^{6}+2 x^{-1}+3 x+2 x^{3}\right) \mathrm{D}-\left(x^{6}-x^{8}\right)$ \\
& $-\left(x^{8}\right)-\left(2 x^{7}\right)$ \\
\hline$d=6$ & $x^{8}\left(6+9 x^{2}+10 x^{4}+3 x^{6}+3 x^{2}\left(x^{2}+x^{4}+x^{6}\right)\right) \mathrm{D}$ \\
\hline$d=5$ & $x^{8}\left(4+7 x^{2}+8 x^{4}+3 x^{6}+x^{3}\left(x^{2}+x^{4}+x^{6}\right)\right) \mathrm{D}$ \\
\hline$d=4$ & $x^{8}\left(2+2 x^{2}+3 x^{4}-x^{6}-x^{8}+1+x^{2}+2 x^{4}-x^{6}-x^{8}\right) \mathrm{D}$ \\
\hline
\end{tabular}

Table 7. Partition function over the space of Lagrangians involving four $R_{\mu \nu \rho \sigma}$ 's (no $R_{\mu \nu}$, no $R$ ). This includes both parity even and parity odd Lagrangians.

the photon and graviton analogues of (4.27) for $D \geq 10$ are easily evaluated by performing the relevant large $D$ computation (see appendix H). Our results are listed in tables 6 and 7 respectively. For $D \leq 9$ we have evaluated the relevant integrals to high order (up to $\mathcal{O}\left(x^{26}\right.$ ) for photons and $\mathcal{O}\left(x^{28}\right)$ for graviton partition functions); the results of these Mathematica experiments are all consistent with particular conjectures for the results for this integral that we present in every dimension in tables 6 and 7 respectively. In [11], photon S-matrix partition function was computed for $D=4$. Our result matches with theirs.

Of course, the conjectures for the results of the plethystic integrals in $D \leq 9$ were not obtained blind but were motivated by physical considerations involving S-matrices which 
we describe in detail in subsequent sections. The power series evaluation of the plethystic performed in this subsection is a direct computational evidence for the correctness of these physically motivated conjectures.

\subsection{Module generators and Lagrangians}

Earlier in this section we presented a detailed discussion of the correspondence between S-matrices and Lagrangians (up to equivalences). Note that the correspondence described so far relates two structures, both of which are $S_{4}$ invariant. Lagrangians built out of identical bosonic fields are automatically $S_{4}$ invariant, while $S$ matrices are $S_{4}$ invariant by construction (see section 2.3 for a detailed discussion).

In our general discussion about the structure of S-matrices in section 2 we found it useful to regard $S_{4}$ invariant $S$ matrices as special members of a larger family of $\mathbb{Z}_{2} \times \mathbb{Z}_{2}$ invariant 'quasi-invariant S-matrices' (see subsection 2.4). Recall, in particular, that it is the space of quasi-invariant polynomial S-matrices (rather than the space of fully $S_{4}$ invariant polynomial $S$ matrices) that form a module. The space of physical (i.e. completely $S_{4}$ invariant) polynomial $S$ matrices is obtained by first enumerating the modules of quasiinvariant S-matrices and then projecting onto the subspace of $S_{3}$ singlets.

As the module structure of local S-matrices plays a key role in their enumeration, it is somewhat unsatisfying to have Lagrangian structures 'dual' only to fully $S_{4}$ invariant S-matrices. In particular, recall that S-matrix modules are labelled by their generators which, in general, transform in non-trivial representations of $S_{3}$. In this brief subsection we describe a procedure that allows us to associate Lagrangians with generators of the local module even when the generators in question are not $S_{3}$ invariant.

Any set of generators $M_{a}$ of the local module (that transform in some representation of $\left.S_{3}\right)$ is naturally associated with an infinite class of genuine ( $S_{3}$ invariant) S-matrices $S\left(M_{a}\right)$ as follows. $S\left(M_{a}\right)$ is defined as the restriction of the span of $M_{a}$ to $S_{3}$ singlets, i.e. restriction to $S_{3}$ singlets of module elements of the form $r . M_{a}$ where $r$ is an element of the ring (i.e. is a polynomial of $s$ and $t$ ). In other words $S\left(M_{a}\right)$ are all the $S_{3}$ invariant descendants of the generators.

Similarly any Lagrangian $L$ can be associated with an infinite class of Lagrangians $C(L)$ defined as follows. $C(L)$ is defined as the set of Lagrangians obtained by taking derivatives of the fields that appear in the Lagrangian and contracting the indices of these derivatives in pairs.

We say that a Lagrangian $L$ is associated with the generators $M_{a}$ if the set of S-matrices obtained from the Lagrangians $C(L)$ coincide with $S\left(M_{a}\right)$. This association allows us to use Lagrangians to label generators (and more generally elements) of the local module. We will use this association in the next section.

As an example consider the photon Lagrangian $\operatorname{Tr}\left(F^{2}\right) \operatorname{Tr}\left(F^{2}\right)$. The corresponding generators of the local Module are $\operatorname{Tr}\left(F_{1} F_{2}\right) \operatorname{Tr}\left(F_{3} F_{4}\right), \operatorname{Tr}\left(F_{1} F_{3}\right) \operatorname{Tr}\left(F_{2} F_{4}\right)$ and $\operatorname{Tr}\left(F_{1} F_{4}\right) \operatorname{Tr}\left(F_{3} F_{2}\right)$; this set of generators transforms in the $\mathbf{3}$ of $S_{3}$. 
For another example consider the photon Lagrangian term $F^{a b} \partial_{a} F^{\mu \nu} \partial_{b} F^{\nu \rho} F^{\rho \mu}$. In this case the generators corresponding to the given Lagrangian consist of the single element $\frac{1}{4}\left(F_{1}^{a b} \partial_{a} F_{2}^{\mu \nu} \partial_{b} F_{3}^{\nu \rho} F_{4}^{\rho \mu}+F_{2}^{a b} \partial_{a} F_{1}^{\mu \nu} \partial_{b} F_{4}^{\nu \rho} F_{3}^{\rho \mu}+F_{3}^{a b} \partial_{a} F_{4}^{\mu \nu} \partial_{b} F_{1}^{\nu \rho} F_{2}^{\rho \mu}+F_{4}^{a b} \partial_{a} F_{3}^{\mu \nu} \partial_{b} F_{2}^{\nu \rho} F_{1}^{\rho \mu}\right)$.

(4.42) had four terms rather than one because no single one of the terms above is $\mathbb{Z}_{2} \times \mathbb{Z}_{2}$ invariant. It is easy to see that the resultant expression (4.42) transforms in the completely symmetric representation of $S_{3}$.

\section{Polynomial photon S-matrices and corresponding Lagrangians}

Recall that quasi-invariant polynomial S-matrices form a module, called the local module. In this section we completely characterize this module by specifying the generators $E_{J}\left(p_{i}, \epsilon_{i}\right)$ for 4-photon S-matrices (the case of graviton S-matrices is the topic of the next section). We also present an explicit parameterization of the physical ( $S_{4}$ invariant) Smatrices that are 'descendants' of these generators and thereby present an explicit parameterization of the most general allowed polynomial four photon and four graviton S-matrix in every dimension. Finally we also present explicit expressions for the Lagrangians from which these S-matrices follow.

Before we dive into the analysis let us spare some time fixing up the notation and convention. In the case of photons, we denote the parity even generators of the local module as $E_{\mathbf{R}}$ and parity odd generators as $O_{\mathbf{R}}$. The subscript $\mathbf{R}$ is either $\mathbf{S}, \mathbf{A}$ or $\mathbf{3}$ denoting its $S_{3}$ representation $\mathbf{1}_{\mathbf{S}}, \mathbf{1}_{\mathbf{A}}$ or $\mathbf{3}$ respectively. When there are multiple generators transforming in the same representation are present, we assign them serial numbers which are also denoted in the subscript. For example, if there two symmetric parity even generators then they are denoted as $E_{\mathbf{S}, 1}$ and $E_{\mathbf{S}, 2}$. In the case when $\mathbf{R}=\mathbf{3}$ or $\mathbf{3}_{\mathbf{A}}$, we use a superscript to denote the specific state of the three dimensional representation (the transformation property of these generators are listed in (2.49) and (2.50)). By convention, we always choose $E_{\mathbf{3}}^{(1)}$ (or $O_{\mathbf{3}}^{(1)}$ ) to be invariant under the swap $1 \leftrightarrow 2$. The second and the third components are obtained by permuting (234) $\rightarrow(342)$. This means, the component (2) is invariant under the swap $1 \leftrightarrow 3$ and the component (3) is invariant under the swap $1 \leftrightarrow 4$. In one case we have to deal with the generator transforming in $\mathbf{3}_{A}$ representation. Recall that this is the representation obtained by acting on a state (1) that is antisymmetric in the exchange of $1 \leftrightarrow 2$ by the cyclic permutation $(234) \rightarrow(342)$. Sometimes we also include the space-time dimension in the superscript when it needs to be emphasized, e.g. $E_{\mathbf{3}}^{D=4,(1)}$.

For gravitons, we have the same notation except that the letters $G$ and $H$ are used, instead of $E$ and $O$, to denote the parity even and parity odd local module generators. In all cases, the corresponding bare module generators are denoted by lower-case letters i.e. the parity even and parity odd bare module generators for photons are denoted as $e$ and $o$ respectively and for gravitons they are denoted as $g$ and $h$ respectively. In order to avoid excessive notation we use the photon notation $E, O$ and $e, o$ for scalars as well. Hopefully this does not cause any confusion as the discussion of the scalar case is very brief and serves as a warm up for the photon and graviton analysis. 
To construct the most general physical (i.e. $S_{4}$ invariant) S-matrix in the span of a quasi-invariant generator, say $E_{\mathbf{R}}$ we need to take the "inner product" with a general polynomial of $(s, t)$ that transforms in the same representation $\mathbf{R}$. For example, if $\mathbf{R}=\mathbf{S}$,

$$
\mathcal{S}=\mathcal{F}^{E_{\mathbf{S}}}(t, u) E_{\mathbf{S}}
$$

where the function $\mathcal{F}^{E_{\mathbf{S}}}(t, u)$ is totally symmetric under $S_{3}$. When $\mathbf{R}=\mathbf{A}$,

$$
\mathcal{S}=\mathcal{F}^{E_{\mathbf{A}}}(t, u) E_{\mathbf{A}}
$$

where the function $\mathcal{F}^{E_{\mathbf{A}}}(t, u)$ is totally antisymmetric under $S_{3}$.

The S-matrix is more involved for quasi-invariant structure that transforms in 3 representation of $S_{3}$. It is given by

$$
\mathcal{S}=\mathcal{F}^{E_{\mathbf{3}}}(t, u) E_{\mathbf{3}}^{(1)}+\mathcal{F}^{E_{\mathbf{3}}}(u, s) E_{\mathbf{3}}^{(2)}+\mathcal{F}^{E_{\mathbf{3}}}(s, t) E_{\mathbf{3}}^{(3)} .
$$

where $\mathcal{F}^{E_{\mathbf{3}}}(t, u)$ is a symmetric function in its two arguments (symmetry under the exchange of $t$ and $u$ is the same as the symmetry under the exchange of $1 \leftrightarrow 2$ which matches with the symmetry of $E_{\mathbf{3}}^{(1)}$ and so on). Sometimes, it helps use the shorthand

$$
\mathcal{F}^{E_{\mathbf{3}}^{(1)}}(t, u) \equiv \mathcal{F}^{E_{\mathbf{3}}}(t, u), \quad \mathcal{F}^{E_{\mathbf{3}}^{(2)}}(t, u) \equiv \mathcal{F}^{E_{\mathbf{3}}}(u, s), \quad \mathcal{F}^{E_{\mathbf{3}}^{(1)}}(t, u) \equiv \mathcal{F}^{E_{3}}(s, t)
$$

So that the above S-matrix can be written as

$$
\mathcal{S}=\sum_{i=1,2,3} \mathcal{F}^{E_{3}^{(i)}}(t, u) E_{\mathbf{3}}^{(i)}
$$

The momenta functions (5.4) transform as noted in (2.49). The S-matrix corresponding to a generator transforming in $\mathbf{3}_{\mathbf{A}}$ representation is also given by the equation (5.3) except that the function $\mathcal{F}$ is antisymmetric rather than symmetric in its two arguments (consequently the momenta functions transform as (2.50)). We will always label the function $\mathcal{F}$ by the quasi-invariant structure that it multiplies.

\subsection{Scalar polynomial S-matrices and corresponding Lagrangians}

As a warm up for the main analysis of this section let us first consider the case of four scalar scattering. As scalar S-matrices don't have any index structures, the local module and the bare module are identical (and so, in particular, are freely generated). In $D \geq 4$, they both are generated by a unique generator $E_{\mathbf{S}}=1$ which is clearly $S_{3}$ invariant (i.e. transforms in the $\mathbf{1}_{\mathbf{S}}$ representation of $S_{3}$ ). The Lagrangian corresponding to this generator is simply $\phi^{4}$.

\subsubsection{Module generators and S-matrix partition functions}

The module analysis of scalar S-matrices (see (2.58)) that the partition function $Z_{\mathrm{S} \text {-matrix }}(x)$ over S-matrices in this case - which we denote by $\mathcal{I}_{\mathrm{s}}^{D \geq 4}(x)$ should simply be given by

$$
\mathcal{I}_{\mathrm{S}}^{D \geq 4}(x)=\mathrm{D}
$$


(see (2.44) for definitions). This prediction is precisely borne out by the partition function obtained via plethystic counting (see table 5 ).

All four scalar scattering amplitudes in $D \geq 4$ are parity invariant. The reason for this is easy to understand. 4-scalar scattering involves only 3 independent vectors (which can be chosen to be any three of the four scattering momenta). It follows that no $D \geq 4$ parity odd S-matrix exists as the number of free indices in the Levi-Civita tensor exceeds the number of independent vectors.

It is clear that the argument of the previous paragraph fails in $D=3$ however. In this case we have the following parity odd structure which is a second generator of the local Module (the first generator continues to be unity)

$$
O_{\mathbf{A}}^{D=3}=\varepsilon_{\mu \nu \rho} k_{1}^{\mu} k_{2}^{\nu} k_{3}^{\rho}
$$

The generator (5.7) is precisely $\widetilde{\varepsilon}$ in (2.19) for $D=3$. The 'Lagrangian' associated with this generator (in the sense of subsection 4.4 ) is, ${ }^{80}$

$$
\varepsilon_{\mu \nu \rho} \partial_{\mu} \phi \partial_{\nu} \phi \partial_{\rho} \phi \phi
$$

Clearly (5.7) transforms in the anti-symmetric representation of $S_{3}$ (see (2.25) )and so it follows from (2.58) that it's contribution to $Z_{\mathrm{S} \text {-matrix }}(x)=\mathcal{I}_{\mathbf{S}}^{D=3}(x)$ is $x^{3} Z_{\mathbf{1}_{\mathbf{A}}}(x)$. It follows that the study of the local S-matrix in this case predicts that

$$
\mathcal{I}_{\mathbf{S}}^{D=3}(x)=Z_{\mathbf{1}_{\mathbf{S}}}(x)+x^{3} Z_{\mathbf{1}_{\mathbf{A}}}(x)=\left(1+x^{9}\right) \mathrm{D}
$$

in perfect agreement with the plethystic result reported in 5 .

In $D=2$, the scalar Lagrangians again are descendants of $\phi^{4}$. This would lead to the expectation that the partition function over Lagrangians should be D. But in two dimensions Mandelstam variables degenerate, either $t$ or $u$ vanish. Hence, the completely symmetric functions of $(s, t, u)$ are generated by only the four derivative generator $s^{2}+$ $t^{2}+u^{2}$. The six derivative generator $s t u$ is identically zero. The partition function over Lagrangians is then, $\left(1-x^{4}\right)^{-1}$. This precisely matches the plethystic counting.

\subsubsection{Explicit expressions for most general S-matrix and corresponding La- grangians}

For completeness we present a completely explicit parameterization of the most general 4 scalar S-matrix and associated Lagrangians. For $D \geq 4$, there is a unique quasi-invariant generator $E_{\mathbf{S}}=1$. The general S-matrix is

$$
\mathcal{S}=\mathcal{F}^{E_{\mathbf{S}}}(t, u)
$$

where this function is completely symmetric under the exchange of $s, t, u$.

\footnotetext{
${ }^{80}$ The Lagrangian (5.8) vanishes for symmetry reasons; however its 'descendants' (Lagrangians obtained by taking derivatives of the four $\phi$ fields in (5.8) and contracting the indices in pairs) do not, in general, vanish. Consequently the Lagrangian (5.8) - while trivial as a functional - is non-trivial as the Lagrangian that labels module generators in the sense of subsection 4.4 .
} 
Recall that $\mathcal{F}^{E_{\mathbf{S}}}(t, u)$ is a polynomial in $t$ and $u$ and so can be expanded as a finite sum of the form

$$
\mathcal{F}^{E_{\mathbf{S}}}(t, u)=\left(\mathcal{F}^{E_{\mathbf{S}}}\right)_{n, m} t^{n} u^{m}
$$

It follows from the analysis of subsection 2.10 that the only S-matrices of the form (5.10) that grow no faster than $s^{2}$ in the Regge limit are

$$
\left.\mathcal{F}^{E_{\mathbf{S}}}(t, u)\right|_{<s^{2}}=a_{0}+a_{4}\left(s^{2}+t^{2}+u^{2}\right)+a_{6}(s t u)
$$

The Lagrangian from which the S-matrix (5.12) follows is proportional to

$$
L^{D \geq 4}=\sum_{m, n}\left(\mathcal{F}^{E_{\mathbf{S}}}\right)_{m, n} 2^{m+n}\left(\prod_{i=1}^{m} \prod_{j=1}^{n}\left(\partial_{\nu_{j}} \partial_{\mu_{i}} \phi\right) \phi \partial_{\mu_{i}} \phi \partial_{\nu_{j}} \phi\right)
$$

Turning now to $D=3$, the most general scalar S-matrix is given by

$$
\mathcal{F}^{E_{\mathbf{S}}^{D=3}}(t, u) E_{\mathbf{S}}^{D=3}+\mathcal{F}^{O} O_{\mathbf{A}=3}^{D=3}(t, u) O_{\mathbf{A}}^{D=3}
$$

where $E_{\mathbf{S}}^{D=3}=1$ and $O_{\mathbf{A}}^{D=3}=\left.\varepsilon_{\mu \nu \rho} k_{1}^{\mu} k_{2}^{\nu} k_{3}^{\rho}\right|_{\mathbb{Z}_{2} \times \mathbb{Z}_{2}}$ as given in equation (5.7). The function $\mathcal{F}^{E_{\mathbf{S}}^{D=3}}(t, u)$ is a general polynomial that is completely symmetric under interchanges of $s, t, u$ while $\mathcal{F}^{O_{\mathrm{A}}^{D=3}}(t, u)$ is a general polynomial that is completely antisymmetric under the same interchanges. We Taylor series expand the functions in (5.14) in a manner completely analogous to (5.11). The Lagrangians from which the S-matrix (5.14) follows is proportional to

$$
\begin{aligned}
L^{D=3}= & \sum_{m, n}\left(\mathcal{F}^{E_{\mathbf{S}}^{D=3}}\right)_{m, n} 2^{m+n}\left(\prod_{i=1}^{m} \prod_{j=1}^{n}\left(\partial_{\nu_{j}} \partial_{\mu_{i}} \phi\right) \phi \partial_{\mu_{i}} \phi \partial_{\nu_{j}} \phi\right) \\
& -i \sum_{m, n}\left(\mathcal{F}^{O_{\mathbf{A}}^{D=3}}\right)_{m, n} 2^{m+n}\left(\prod_{i=1}^{m} \prod_{j=1}^{n} \epsilon_{\alpha \beta \gamma}\left(\partial_{\nu_{j}} \partial_{\mu_{i}} \partial^{\alpha} \phi\right) \partial^{\beta} \phi \partial_{\mu_{i}} \partial^{\gamma} \phi \partial_{\nu_{j}} \phi\right)
\end{aligned}
$$

\subsection{Construction of all parity even photon S-matrices for $D \geq 5$}

We now turn to the main topic of this section - the study of polynomial photon S-matrices. In this subsection we begin this analysis by presenting an explicit construction of all parity even S-matrices in $D \geq 5$.

In appendix I, we have painstakingly shown that the most general parity even gauge invariant Lagrangian can be obtained by taking linear combinations of pairs of contracted derivatives on the three 'generator' Lagrangians

$$
\operatorname{Tr}\left(F^{2}\right) \operatorname{Tr}\left(F^{2}\right), \quad \operatorname{Tr}\left(F^{4}\right), \quad-F^{a b} \partial_{a} F^{\mu \nu} \partial_{b} F^{\nu \rho} F^{\rho \mu}
$$


The generators of the local module dual to these Lagrangians (in the sense of section 4.4) are given by

$$
\begin{aligned}
E_{\mathbf{3}, 1}^{(1)}= & 8 \operatorname{Tr}\left(F_{1} F_{2}\right) \operatorname{Tr}\left(F_{3} F_{4}\right), \quad E_{\mathbf{3}, 1}^{(2)}=8 \operatorname{Tr}\left(F_{1} F_{3}\right) \operatorname{Tr}\left(F_{2} F_{4}\right), \quad E_{\mathbf{3}, 1}^{(3)}=8 \operatorname{Tr}\left(F_{1} F_{4}\right) \operatorname{Tr}\left(F_{3} F_{2}\right), \\
E_{\mathbf{3}, 2}^{(1)}= & 8 \operatorname{Tr}\left(F_{1} F_{3} F_{2} F_{4}\right), \quad E_{\mathbf{3}, 2}^{(2)}=8 \operatorname{Tr}\left(F_{1} F_{2} F_{3} F_{4}\right), \quad E_{\mathbf{3}, 2}^{(3)}=8 \operatorname{Tr}\left(F_{1} F_{3} F_{4} F_{2}\right), \\
E_{\mathbf{S}} \simeq & -\left.6 F_{1}^{a b} \partial_{a} F_{2}^{\mu \nu} \partial_{b} F_{3}^{\nu \rho} F_{4}^{\rho \mu}\right|_{\mathbb{Z}_{2} \times \mathbb{Z}_{2}} \\
= & 6\left(-F_{1}^{a b} \partial_{a} F_{2}^{\mu \nu} \partial_{b} F_{3}^{\nu \rho} F_{4}^{\rho \mu}-F_{2}^{a b} \partial_{a} F_{1}^{\mu \nu} \partial_{b} F_{4}^{\nu \rho} F_{3}^{\rho \mu}\right. \\
& \left.\quad-F_{3}^{a b} \partial_{a} F_{4}^{\mu \nu} \partial_{b} F_{1}^{\nu \rho} F_{2}^{\rho \mu}-F_{4}^{a b} \partial_{a} F_{3}^{\mu \nu} \partial_{b} F_{2}^{\nu \rho} F_{1}^{\rho \mu}\right) .
\end{aligned}
$$

We have demonstrated that quantities listed in (5.17) are the generators of the parity even part of the local Module in every dimension. Note that there are two four derivative generators in the $\mathbf{3}$ and one six derivative generators in the $\mathbf{1}_{\mathbf{S}}$ of $S_{3}$. The second subscript on $E_{\mathbf{3}}$ is simply an arbitrarily assigned serial number.

It remains to check whether the parity even part of the local module is freely generated. Note that the local module has 7 generators in every dimension. From table 3 we see that the number of generators of the parity even part of the bare module is 5 in $D=4$ and 1 in $D=3$. As the number of generators of the local Module exceeds the number of generators of the bare module, it follows (see subsubsection 2.4.3) that the local Module is not freely generated in these dimensions. In $D=4$ and 3 the local parity even S-matrix module is not completely specified by their generators (5.17); we also need to specify the relations obeyed within the modules generated by these generators. We will return to this point later in this subsection.

In $D \geq 5$, on the other hand, we see from table 3 that the bare module has exactly as many generators as the local module (the representations of these generators also match those of the local module: two in the $\mathbf{3}$ and one in the $\mathbf{1}_{\mathbf{S}}$ ). It follows that the local module is freely generated unless the extremely stringent condition (2.32) is satisfied. We will demonstrate below that (2.32) is not satisfied so that the local module is also freely generated. In this case, therefore, parity even local S-matrix module - and so the space of all local parity even S-matrices - is completely specified by the generators (5.17).

To proceed we express local module generators (5.17) in terms of the generators of the bare modules $e_{I}\left(\alpha_{i}, \epsilon_{i}^{\perp}\right)$ that are constructed in section 3.2 ,

$$
\begin{aligned}
& e_{\mathbf{3}, 1}^{(1)}\left(\alpha_{i}, \epsilon_{i}^{\perp}\right)=\left(\epsilon_{1}^{\perp} \cdot \epsilon_{2}^{\perp}\right)\left(\epsilon_{3}^{\perp} \cdot \epsilon_{4}^{\perp}\right), \quad \quad e_{3,1}^{(2)}\left(\alpha_{i}, \epsilon_{i}^{\perp}\right)=\left(\epsilon_{1}^{\perp} \cdot \epsilon_{3}^{\perp}\right)\left(\epsilon_{2}^{\perp} \cdot \epsilon_{4}^{\perp}\right), \\
& e_{3,1}^{(3)}\left(\alpha_{i}, \epsilon_{i}^{\perp}\right)=\left(\epsilon_{1}^{\perp} \cdot \epsilon_{4}^{\perp}\right)\left(\epsilon_{3}^{\perp} \cdot \epsilon_{2}^{\perp}\right) \\
& e_{\mathbf{3}, 2}^{(1)}\left(\alpha_{i}, \epsilon_{i}^{\perp}\right)=\left(\epsilon_{1}^{\perp} \cdot \epsilon_{2}^{\perp} \alpha_{3} \alpha_{4}+\epsilon_{3}^{\perp} . \epsilon_{4}^{\perp} \alpha_{1} \alpha_{2}\right), \quad e_{3,2}^{(2)}\left(\alpha_{i}, \epsilon_{i}^{\perp}\right)=\left(\epsilon_{1}^{\perp} \cdot \epsilon_{3}^{\perp} \alpha_{2} \alpha_{4}+\epsilon_{2}^{\perp} \cdot \epsilon_{4}^{\perp} \alpha_{1} \alpha_{3}\right), \\
& e_{3,2}^{(3)}\left(\alpha_{i}, \epsilon_{i}^{\perp}\right)=\left(\epsilon_{1}^{\perp} \cdot \epsilon_{4}^{\perp} \alpha_{3} \alpha_{2}+\epsilon_{3}^{\perp} . \epsilon_{2}^{\perp} \alpha_{1} \alpha_{4}\right) \\
& e_{\mathbf{S}}\left(\alpha_{i}, \epsilon_{i}^{\perp}\right)=\alpha_{1} \alpha_{2} \alpha_{3} \alpha_{4} \text {. }
\end{aligned}
$$


As explicitly indicated $e_{\mathbf{3}, 1}$ and $e_{\mathbf{3}, 2}$ transform in $\mathbf{3}$ of $S_{3}$. We have: ${ }^{81}$

$$
\begin{aligned}
E_{\mathbf{3}, 1}^{(1)} & =-8 s^{2} e_{\mathbf{3}, 1}^{(1)}+8 s^{2} e_{\mathbf{3}, 2}^{(1)}-8 s^{2} e_{\mathbf{S}}, \quad E_{\mathbf{3}, 1}^{(2)}=-8 t^{2} e_{\mathbf{3}, 1}^{(2)}+8 t^{2} e_{\mathbf{3}, 2}^{(2)}-8 t^{2} e_{\mathbf{S}}, \\
E_{\mathbf{3}, 1}^{(3)} & =-8 u^{2} e_{\mathbf{3}, 1}^{(3)}+8 u^{2} e_{\mathbf{3}, 2}^{(3)}-8 u^{2} e_{\mathbf{S}}, \\
E_{\mathbf{3}, 2}^{(1)} & =-2\left(u^{2} e_{\mathbf{3}, 1}^{(2)}+t^{2} e_{\mathbf{3}, 1}^{(3)}\right)+2\left(u(s-t) e_{\mathbf{3}, 2}^{(2)}+t(s-u) e_{\mathbf{3}, 2}^{(3)}\right)-2\left(t^{2}+u^{2}\right) e_{\mathbf{S}}, \\
E_{\mathbf{3}, 2}^{(2)} & =-2\left(s^{2} e_{\mathbf{3}, 1}^{(3)}+u^{2} e_{\mathbf{3}, 1}^{(1)}\right)+2\left(s(t-u) e_{\mathbf{3}, 2}^{(3)}+u(t-s) e_{\mathbf{3}, 2}^{(1)}\right)-2\left(u^{2}+s^{2}\right) e_{\mathbf{S}}, \\
E_{\mathbf{3}, 2}^{(3)} & =-2\left(t^{2} e_{\mathbf{3}, 1}^{(1)}+s^{2} e_{\mathbf{3}, 1}^{(2)}\right)+2\left(t(u-s) e_{\mathbf{3}, 2}^{(1)}+s(u-t) e_{\mathbf{3}, 2}^{(2)}\right)-2\left(s^{2}+t^{2}\right) e_{\mathbf{S}}, \\
E_{\mathbf{S}} & =3 s t u\left(e_{\mathbf{3}, 2}^{(1)}+e_{\mathbf{3}, 2}^{(2)}+e_{\mathbf{3}, 2}^{(3)}-2 e_{\mathbf{S}}\right) .
\end{aligned}
$$

With the explicit expressions (5.19) in hand it is not difficult to check that (2.32) is indeed not obeyed and the local module is generated freely by the above generators (from (5.19), we find, Det $\left.\left[p_{I J}(s, t)\right]=393216 s^{5} t^{5} u^{5}\right)$. It follows that (5.23) is indeed the expected answer for the partition function over S-matrices.

For completeness we present an explicit parameterization of the most general parity even S-matrix for four photon scattering in $D \geq 5$ and also of the Lagrangians that generate these S-matrices. The most general S-matrix is parameterized by two $\mathbb{Z}_{2}$ symmetric functions of $t$ and $u$, and one completely $s, t, u$ symmetric function. Explicitly we have

$$
\begin{aligned}
\mathcal{S}_{\text {even }}^{D \geq 5}=4( & \mathcal{F}^{E_{3,1}}(t, u)\left(p_{\mu}^{1} \epsilon_{\nu}^{1}-p_{\nu}^{1} \epsilon_{\mu}^{1}\right)\left(p_{\mu}^{2} \epsilon_{\nu}^{2}-p_{\nu}^{2} \epsilon_{\mu}^{2}\right)\left(p_{\alpha}^{3} \epsilon_{\beta}^{3}-p_{\beta}^{3} \epsilon_{\alpha}^{3}\right)\left(p_{\alpha}^{4} \epsilon_{\beta}^{4}-p_{\beta}^{4} \epsilon_{\alpha}^{4}\right) \\
& +\mathcal{F}^{E_{3,1}}(u, s)\left(p_{\mu}^{1} \epsilon_{\nu}^{1}-p_{\nu}^{1} \epsilon_{\mu}^{1}\right)\left(p_{\mu}^{3} \epsilon_{\nu}^{3}-p_{\nu}^{3} \epsilon_{\mu}^{3}\right)\left(p_{\alpha}^{2} \epsilon_{\beta}^{2}-p_{\beta}^{2} \epsilon_{\alpha}^{2}\right)\left(p_{\alpha}^{4} \epsilon_{\beta}^{4}-p_{\beta}^{4} \epsilon_{\alpha}^{4}\right) \\
& \left.+\mathcal{F}^{E_{3,1}}(s, t)\left(p_{\mu}^{1} \epsilon_{\nu}^{1}-p_{\nu}^{1} \epsilon_{\mu}^{1}\right)\left(p_{\mu}^{4} \epsilon_{\nu}^{4}-p_{\nu}^{4} \epsilon_{\mu}^{4}\right)\left(p_{\alpha}^{3} \epsilon_{\beta}^{3}-p_{\beta}^{3} \epsilon_{\alpha}^{3}\right)\left(p_{\alpha}^{2} \epsilon_{\beta}^{2}-p_{\beta}^{2} \epsilon_{\alpha}^{2}\right)\right) \\
+ & 4\left(\mathcal{F}^{E_{3,2}}(t, u)\left(p_{\mu}^{1} \epsilon_{\nu}^{1}-p_{\nu}^{1} \epsilon_{\mu}^{1}\right)\left(p_{\nu}^{3} \epsilon_{\alpha}^{3}-p_{\alpha}^{3} \epsilon_{\nu}^{3}\right)\left(p_{\alpha}^{2} \epsilon_{\beta}^{2}-p_{\beta}^{2} \epsilon_{\alpha}^{2}\right)\left(p_{\beta}^{4} \epsilon_{\mu}^{4}-p_{\mu}^{4} \epsilon_{\beta}^{4}\right)\right. \\
& +\mathcal{F}^{E_{3,2}}(u, s)\left(p_{\mu}^{1} \epsilon_{\nu}^{1}-p_{\nu}^{1} \epsilon_{\mu}^{1}\right)\left(p_{\nu}^{2} \epsilon_{\alpha}^{2}-p_{\alpha}^{2} \epsilon_{\nu}^{2}\right)\left(p_{\alpha}^{3} \epsilon_{\beta}^{3}-p_{\beta}^{3} \epsilon_{\alpha}^{3}\right)\left(p_{\beta}^{4} \epsilon_{\mu}^{4}-p_{\mu}^{4} \epsilon_{\beta}^{4}\right) \\
& \left.+\mathcal{F}^{E_{3,2}}(s, t)\left(p_{\mu}^{1} \epsilon_{\nu}^{1}-p_{\nu}^{1} \epsilon_{\mu}^{1}\right)\left(p_{\nu}^{3} \epsilon_{\alpha}^{3}-p_{\alpha}^{3} \epsilon_{\nu}^{3}\right)\left(p_{\alpha}^{4} \epsilon_{\beta}^{4}-p_{\beta}^{4} \epsilon_{\alpha}^{4}\right)\left(p_{\beta}^{2} \epsilon_{\mu}^{2}-p_{\mu}^{2} \epsilon_{\beta}^{2}\right)\right) \\
+ & \mathcal{F}^{E_{\mathbf{S}}}(t, u) \\
\times & \left(\left(p_{a}^{1} \epsilon_{b}^{1}-p_{b}^{1} \epsilon_{a}^{1}\right) p_{a}^{2}\left(p_{\mu}^{2} \epsilon_{\nu}^{2}-p_{\nu}^{2} \epsilon_{\mu}^{2}\right) p_{b}^{3}\left(p_{\nu}^{3} \epsilon_{\alpha}^{3}-p_{\alpha}^{3} \epsilon_{\nu}^{3}\right)\left(p_{\alpha}^{4} \epsilon_{\mu}^{4}-p_{\mu}^{4} \epsilon_{\alpha}^{4}\right)\right. \\
& +\left(p_{a}^{2} \epsilon_{b}^{2}-p_{b}^{2} \epsilon_{a}^{2}\right) p_{a}^{1}\left(p_{\mu}^{1} \epsilon_{\nu}^{1}-p_{\nu}^{1} \epsilon_{\mu}^{1}\right) p_{b}^{4}\left(p_{\nu}^{4} \epsilon_{\alpha}^{4}-p_{\alpha}^{4} \epsilon_{\nu}^{4}\right)\left(p_{\alpha}^{3} \epsilon_{\mu}^{3}-p_{\mu}^{3} \epsilon_{\alpha}^{3}\right) \\
& +\left(p_{a}^{3} \epsilon_{b}^{3}-p_{b}^{3} \epsilon_{a}^{3}\right) p_{a}^{4}\left(p_{\mu}^{4} \epsilon_{\nu}^{4}-p_{\nu}^{4} \epsilon_{\mu}^{4}\right) p_{b}^{1}\left(p_{\nu}^{1} \epsilon_{\alpha}^{1}-p_{\alpha}^{1} \epsilon_{\nu}^{1}\right)\left(p_{\alpha}^{2} \epsilon_{\mu}^{2}-p_{\mu}^{2} \epsilon_{\alpha}^{2}\right) \\
& \left.+\left(p_{a}^{4} \epsilon_{b}^{4}-p_{b}^{4} \epsilon_{a}^{4}\right) p_{a}^{3}\left(p_{\mu}^{3} \epsilon_{\nu}^{3}-p_{\nu}^{3} \epsilon_{\mu}^{3}\right) p_{b}^{2}\left(p_{\nu}^{2} \epsilon_{\alpha}^{2}-p_{\alpha}^{2} \epsilon_{\nu}^{2}\right)\left(p_{\alpha}^{1} \epsilon_{\mu}^{1}-p_{\mu}^{1} \epsilon_{\alpha}^{1}\right)\right)
\end{aligned}
$$

The functions $\mathcal{F}^{E_{\mathbf{3}, 1}}(t, u), \mathcal{F}^{E_{\mathbf{3}, 2}}(t, u)$ are each arbitrary functions that are symmetric in their two arguments. These functions with permuted arguments transform in the $\mathbf{3}$ of

\footnotetext{
${ }^{81}$ If $\alpha^{(i)}$ and $\beta^{(j)}$ transform in the 3 representation, then $\theta^{(i)}=\alpha^{(i)} \beta^{(i)}, \phi^{(1)}=\alpha^{(3)} \beta^{(2)}+$ $\alpha^{(2)} \beta^{(3)}$ and cyclic, and $\zeta^{(1)}=\alpha^{(1)}\left(\beta^{(2)}+\beta^{(3)}\right)$ and cyclic all also transform in the 3 representation. The reader can use these rules - together with the fact that $(s, t, u)$ transform in $\mathbf{2}_{\mathrm{M}}$ and the triplets of functions $\left(s^{2}, t^{2}, u^{2}\right),(s t, t u, u s)$ transform in the $\mathbf{3}$ - to show that the expressions that appear on the r.h.s. of the definitions of $E_{1}^{(i)}$ and $E_{2}^{(i)}$ transform in the $\mathbf{3}$.
} 
$S_{3}$ (see the discussion around (2.46)) explaining the superscript 3 on these functions. On the other hand $\mathcal{F}^{E_{\mathbf{S}}}(t, u)$ is a function that is completely symmetric under interchange of $s, t$ and $u$.

It is not difficult to verify (see appendix $\mathrm{J}$ ) that the most general S-matrix of the form (5.20) that grows no faster than $s^{2}$ in the Regge limit is given by the four parameter set

$$
\mathcal{F}^{E_{3,1}}(t, u)=c_{1}, \quad \mathcal{F}^{E_{3,2}}(t, u)=c_{2}+c_{3}(u+t), \quad \mathcal{F}^{E_{\mathbf{S}}}(t, u)=c_{4} .
$$

The S-matrices parameterized by $c_{1}$ and $c_{2}$ are both four derivative. The S-matrices parameterized by $c_{3}$ and $c_{4}$ are both 6 derivatives. All 4 S-matrices corresponding to $c_{i}$, $i=1,2,3,4$ grow like $s^{2}$ in the Regge limit.

The three functions $\mathcal{F}^{E_{\mathbf{3}, 1}}(t, u), \mathcal{F}^{E_{\mathbf{3}, 2}}(t, u)$ and $\mathcal{F}^{E_{\mathbf{S}}}(t, u)$ can be Taylor expanded in a manner completely analogous to (5.11). The Lagrangians that generates the S-matrix (5.20) is given by

$$
\begin{aligned}
L_{\text {even }}^{D \geq 5}= & \sum_{m, n}\left(\mathcal{F}^{E_{3,1}}\right)_{m, n} 2^{m+n}\left(\prod_{i=1}^{m} \prod_{j=1}^{n} \operatorname{Tr}\left(\partial_{\nu_{j}} \partial_{\mu_{i}} F F\right) \operatorname{Tr}\left(\partial_{\mu_{i}} F \partial_{\nu_{j}} F\right)\right) \\
& +\sum_{m, n}\left(\mathcal{F}^{E_{3,2}}\right)_{m, n} 2^{m+n}\left(\prod_{i=1}^{m} \prod_{j=1}^{n} \operatorname{Tr}\left(\partial_{\nu_{j}} \partial_{\mu_{i}} F \partial_{\mu_{i}} F F \partial_{\nu_{j}} F\right)\right) \\
& +\sum_{m, n}\left(\mathcal{F}^{E_{\mathbf{S}}}\right)_{m, n} 2^{m+n}\left(-\prod_{i=1}^{m} \prod_{j=1}^{n} \partial_{\mu_{i}} \partial_{\nu_{j}} F_{a b} \operatorname{Tr}\left(\partial_{\mu_{i}} \partial_{a} F \partial_{\nu_{j}} \partial_{b} F F\right)\right) .
\end{aligned}
$$

As mentioned above (5.20) and (5.22) describe the most general polynomial S-matrix (and corresponding local Lagrangian) for parity even four photon scattering in dimensions $D \geq 5$. In these dimensions the three functions label polynomial S-matrices in a one to one manner; every distinct choice of these functions yields a distinct S-matrix, and every polynomial S-matrix corresponds to some choice of these functions.

In fact the expressions (5.20) and (5.22) also apply to $D=4$ and $D=3$. In this case, however, the map between the functions $\mathcal{F}^{E_{\mathbf{3}, 1}}(t, u), \mathcal{F}^{E_{\mathbf{3}, 2}}(t, u)$ and $\mathcal{F}^{E_{\mathbf{S}}}(t, u)$ and polynomial S-matrices is many to one. While every S-matrix continues to correspond to some choice of these three functions, many different choices of these functions yield the same local S-matrix (this is another way of saying that the parity odd local S-matrix module in these dimensions is not freely generated but has relations).

We now turn to a brief discussion of parity odd S-matrices, i.e. S-matrices that use a single copy of the Levi-Civita tensor. As this tensor has a different numbers of indices in different dimensions, the structure of the parity odd local module tends to be very specific to dimension. However there is one universal statement about parity odd S-matrices that is easy to make, namely that no such S-matrices exist for $D \geq 8$. This simple fact follows from the observation that in these the Levi-Civita tensor has 8 or more indices but only 7 independent vectors - three momenta and four polarizations - for these indices to contract with. It follows that all four photon S-matrices are parity even in $D \geq 8$ (this fact is also clear from table 3). 
In the rest of this subsection we will use the discussion above to understand the detailed structure of the local S-matrix module, the partition function over S-matrices, and also provide a completely explicit parameterization of S-matrices and their corresponding Lagrangians dimension by dimension. In order to do this we will construct the parity odd local S-matrix modules in the dimensions in which they exist. We will also completely characterize the relations in the local parity even S-matrix module in $D=4$ and $D=3$. Finally we will reconcile our results with the explicit results of plethystic counting presented in table 6 .

Finally a notational remark: in the rest of this subsection we use the notation $I_{\mathrm{v}}^{D=m}(x)$ for the partition function $Z_{\mathrm{S} \text {-matrix }}($ see $(2.56)$ ) for the case of 4 photon scattering in $m$ dimensions.

\section{$5.3 \quad D \geq 8$}

In these dimensions all S-matrices are parity even. The local module of parity even Smatrices is freely generated. The module (see (5.17)) has two 4 derivative generators both in the $\mathbf{3}$ and one 6 derivative generator in the $\mathbf{1}_{\mathbf{S}}$.

It follows from (2.57) that the partition function (2.56) over S-matrices in these dimensions is given by

$$
I_{\mathrm{v}}^{D \geq 8}(x)=2 x^{4} Z_{\mathbf{3}}(x)+x^{6} Z_{\mathbf{1}_{\mathbf{S}}}(x) .
$$

It remains to compare the prediction (5.23) against the explicit results of plethystic counting presented in table 6 . For $D \geq 10$ the results reported in 6 match exactly with the prediction (5.23); we view this matching as a highly non-trivial confirmation of (5.23).

It is at first puzzling, however, that the result of Plethystic 6 differs from (5.23) by $-x^{5}$ in $D=9$ and $+x^{4}$ in $D=8$. The resolution to this apparent contradiction is that the plethystic procedure we adopted in 4.3 slightly miscounts the S-matrices in $D=9$ and $D=8$ as we now explain.

Recall that the plethystic counting procedure of section 4.3 proceed in three steps.

- Step 1: we computed the partition function over operators built out of four (gauge invariant and on-shell) letters.

- Step 2: we organized four letter operators into derivatives of primaries. Assuming descendants were freely generated we obtained a partition function over primaries (non total derivatives ) by multiplying the result of Step 1 by $\mathrm{D}^{-1}$.

- Step 3: we integrated the result of Step 2 over the $\mathrm{SO}(D)$ group with the Haar measure to isolate $\mathrm{SO}(D)$ singlet primaries.

In $D=9$ the error in the plethystic procedure lies in the assumption in step 2 that derivative descendants are freely generated for all primaries. This assumption fails for one primary, namely $J=* F \wedge F \wedge F \wedge F$. $J^{\mu}$ is an identically conserved current (this follows from use of the Bianchi identity) so that $\partial_{\mu} J^{\mu}=0$. Of course it is also true that $\partial_{\alpha_{1}} \partial_{\alpha_{2}} \ldots\left(\partial_{\mu} J^{\mu}\right)=0$. Borrowing language from the representation theory of the conformal algebra, $J_{\mu}$ is the primary of a 'short representation' and $\partial_{\mu} J^{\mu}$ is a primary null state. It 
follows that the contribution derivatives of $J_{\mu}$ to the final result of Step 2 is

$$
x^{4} \chi_{\square}-x^{5}
$$

where $\chi_{V}$ is the $\mathrm{SO}(9)$ character of the vector and $x^{5}$ multiplies unity, the character of the scalar. It follows that the contribution of this primary to the integral over the $\mathrm{SO}(9)$ gauge group in Step 3 is $-x^{5}$. Removing this fake contribution (by adding $x^{5}$ ) turns the $D=9$ entry of table 6 into the correct module prediction (5.23).

In $D=8$ the plethystic counting makes the opposite error; it omits to recognize that the quantity $F \wedge F \wedge F \wedge F$ is a total derivative. The reason for this failing is that the plethystic procedure (Step 2 above) only removes total derivatives of quartic polynomials built out of field strength letters. However $F \wedge F \wedge F \wedge F$ is the total derivative of the 7 dimensional Chern Simons form which is not a polynomial in gauge invariant letters. In order to get the correct S-matrix partition function in $D=8$ we must, consequently, remove this total derivative by hand, i.e. subtract $x^{4}$ from the $D=8$ entry of table 6 . Once again this procedure yields the correct module prediction (5.23). ${ }^{82}$

In summary, the correct partition function over S-matrices is given by (5.23) for all $D \geq$ 8. The most general S-matrix in these dimensions is given by (5.20) and the Lagrangian that generates this S-matrix continues to be given by (5.22).

\section{$5.4 \quad D=7$}

In this case the plethystic partition function reported in table 6 can be recast as

$$
2 x^{4} Z_{\mathbf{3}}(x)+x^{6} Z_{\mathbf{1}_{\mathbf{S}}}(x)+x^{3} Z_{\mathbf{1}_{\mathbf{S}}}(x)-x^{3} .
$$

Once again the plethystic counting in $D=7$ makes a small error; it omits to count the 3 derivative gauge invariant Lagrangian,

$$
O_{\mathbf{S}}^{D=7}=\mathrm{CS}_{7}=*(A \wedge F \wedge F \wedge F)
$$

the 7 dimensional Chern Simons form, as this expression is not a polynomial in gauge invariant letters. ${ }^{83}$ This error is corrected for by adding $x^{3}$ to $(5.29)$ and so the corrected Plethystic result predicts that

$$
I_{\mathrm{v}}^{D=7}(x)=2 x^{4} Z_{\mathbf{3}}(x)+x^{6} Z_{\mathbf{1}_{\mathbf{S}}}(x)+x^{3} Z_{\mathbf{1}_{\mathbf{S}}}(x)
$$

\footnotetext{
${ }^{82}$ Operators formed out of $F \wedge F \wedge F \wedge F$ by taking derivatives of the four field strengths and contracting indices in pairs are also total derivatives. however it is not difficult to check that such operators can be written as total derivatives of operators that are quartic in letters and so are correctly subtracted out by the plethystic procedure. For example consider $\epsilon^{a b c d e f g h} \partial_{\mu} F_{a b} \partial^{\mu} F_{c d} F_{e f} F_{g h}$. We write this as,

$$
\begin{aligned}
\epsilon^{a b c d e f g h} \partial_{\mu} F_{a b} \partial^{\mu} F_{c d} F_{e f} F_{g h}= & -2 \epsilon^{a b c d e f g h} \partial_{a} F_{b \mu} \partial^{\mu} F_{c d} F_{e f} F_{g h} \\
= & -2 \epsilon^{a b c d e f g h} \partial_{a}\left(F_{b \mu} \partial^{\mu} F_{c d} F_{e f} F_{g h}\right)+2 \epsilon^{a b c d e f g h} F_{b \mu} \partial^{\mu} \partial_{a} F_{c d} F_{e f} F_{g h} \\
& +2 \epsilon^{a b c d e f g h} F_{b \mu} \partial^{\mu} F_{c d} \partial_{a} F_{e f} F_{g h}+2 \epsilon^{a b c d e f g h} F_{b \mu} \partial^{\mu} F_{c d} F_{e f} \partial_{a} F_{g h} \\
= & -2 \epsilon^{a b c d e f g h} \partial_{a}\left(F_{b \mu} \partial^{\mu} F_{c d} F_{e f} F_{g h}\right)
\end{aligned}
$$

where in going from the first line to the second line we have used Bianchi identity.

${ }^{83}$ On the other hand Lagrangians formed out of $A \wedge F \wedge F \wedge F$ by taking derivatives and contracting indices in pairs can be written entirely out of field strengths - up to total derivatives — and so are correctly
} 
Note that in $D=7$ (and all odd dimensions), contributions to the S-matrix partition function even in $x$ count parity even S-matrices while contributions that are odd in $x$ count parity odd structures ${ }^{84}$ It follows that (5.29) can be refined into

$$
I_{\mathrm{v}}^{D=7 \text { even }}=x^{4}\left(2+3 x^{2}+2 x^{4}\right) \mathrm{D}, \quad I_{\mathrm{v}}^{\mathrm{D}=7 \text { odd }}=x^{3} \mathrm{D} .
$$

The parity even part of the prediction (5.30) is precisely the partition function of a freely generated module with generators (5.17). As described earlier in this subsection, this is the expected structure of the local module for parity even S-matrices in this dimension. This agreement is non-trivial confirmation of the module prediction that the most general local parity even S-matrix in $D=7$ continues to be given by (5.20) and the Lagrangian that generates this S-matrix continues to be given by (5.22).

We now turn to the study of the parity odd S-matrix module. In this case the parity odd bare module has a single generator in the $\mathbf{1}_{\mathbf{S}}$ which in fact coincides with the single parity odd generator of the local module; the generator in question is given by

$$
O_{\mathbf{S}}^{D=7}=\epsilon_{1} \wedge \epsilon_{2} \wedge \epsilon_{3} \wedge \epsilon_{4} \wedge k_{1} \wedge k_{2} \wedge k_{3}
$$

and the corresponding Lagrangian is the 7 dimensional Chern Simons form that we have already encountered above. As this generator has derivative dimension 3 its contribution to the S-matrix partition function precisely agrees with (5.30). We thus have a complete 'module' explanation for the corrected plethystic result (5.30).

The most general parity odd S-matrix in $D=7$, is given by

$$
\mathcal{S}_{\text {odd }}^{D=7}=-i 4 \mathcal{F}^{O \mathrm{~S}=7}(t, u)\left(8 *\left(\epsilon^{1} \wedge p^{2} \wedge \epsilon^{2} \wedge p^{3} \wedge \epsilon^{3} \wedge p^{4} \wedge \epsilon^{4}\right)\right)
$$

where, as $\mathbf{S}$ in superscript suggests, the function $\mathcal{F}_{\mathbf{S}}^{D=7}(t, u)$ is an arbitrary completely symmetric function of $s, t, u$. This function $\mathcal{F}^{O \mathrm{~S}=7}(t, u)$ can be expanded as in (5.11). The parity odd Lagrangian from which (5.32) follows takes the form

$$
L_{\text {odd }}^{D=7}=\sum_{m, n}\left(\mathcal{F}^{O \mathrm{~S}=7}\right)_{m, n} 2^{m+n}\left(\prod_{i=1}^{m} \prod_{j=1}^{n}\left(\partial_{\nu_{j}} \partial_{\mu_{i}} A \wedge \partial_{\mu_{i}} F \wedge \partial_{\nu_{j}} F \wedge F\right)\right)
$$

There is exactly one parity odd photonic S-matrix that grows no faster than $s^{2}$ in the Regge limit; this is the S-matrix with $\mathcal{F}^{O{ }_{\mathrm{S}}^{D=7}}(t, u)=$ const. Explicitly the S-matrix is given counted by the plethystic procedure. For example consider $\epsilon^{b c d e f g h} \partial_{\mu} A_{b} \partial^{\mu} F_{c d} F_{e f} F_{g h}$

$$
\begin{aligned}
\epsilon^{b c d e f g h} \partial^{\mu} A_{b} \partial_{\mu} F_{c d} F_{e f} F_{g h}= & -2 \epsilon^{b c d e f g h} \partial^{\mu} A_{b} \partial_{c} F_{d \mu} F_{e f} F_{g h} \\
= & -2 \epsilon^{b c d e f g h} \partial_{c}\left(\partial^{\mu} A_{b} F_{d \mu} F_{e f} F_{g h}\right)+2 \epsilon^{b c d e f g h} \partial_{\mu} F_{c b} F_{d \mu} F_{e f} F_{g h} \\
& +2 \epsilon^{b c d e f g h} \partial^{\mu} A_{b} F_{d \mu} \partial_{c} F_{e f} F_{g h}+2 \epsilon^{b c d e f g h} \partial^{\mu} A_{b} F_{d \mu} F_{e f} \partial_{c} F_{g h} \\
= & -2 \epsilon^{b c d e f g h} \partial_{c}\left(\partial^{\mu} A_{b} F_{d \mu} F_{e f} F_{g h}\right)+2 \epsilon^{b c d e f g h} \partial_{\mu} F_{c b} F_{d \mu} F_{e f} F_{g h}
\end{aligned}
$$

where in going from the first to second step we have used Bianchi identity and we have removed the last two terms in the second line using Bianchi identity again.

${ }^{84}$ This follows immediately from the fact that the Levi-Civita symbol has an odd number of indices when $D$ is odd. When $D$ is even, on the other hand, parity odd and parity even S-matrices both yield even (in $x$ ) contributions to the S-matrix partition function. 
by (5.31) - the generator of the local module which, in this case, also happens to be the generator of the bare module. This momentum dependence of this S-matrix is $\sqrt{s t u}$ and so it scales like $s$ in the Regge limit.

\section{$5.5 D=6$}

In this case the plethystic partition function reported in table 6 can be recast as

$$
2 x^{4} Z_{\mathbf{3}}(x)+x^{6} Z_{\mathbf{1}_{\mathbf{S}}}(x)+x^{6} Z_{\mathbf{1}_{\mathbf{A}}}(x)
$$

In this case it turns out that the plethystic counting makes no errors, and (5.34) is the correct formula for the partition function over S-matrices. The analysis of parity even module structures presented earlier in this subsection predicts that the parity even part of the partition function over S-matrices is given by (5.23) for all $D \geq 5$, and so, in particular, for $D=6$. Comparing with (5.34) it follows that

$$
I_{\mathrm{v}}^{D=6, \text { even }}=2 x^{4} Z_{\mathbf{3}}(x)+x^{6} Z_{\mathbf{1}_{\mathbf{S}}}(x), \quad I_{\mathrm{v}}^{D=6, \text { odd }}=x^{6} Z_{\mathbf{1}_{\mathbf{A}}}(x) .
$$

The even part of the S-matrix is no different from higher dimensions; in particular the most general parity even polynomial S-matrix continues to be given by (5.20) and the Lagrangian that generates this S-matrix continues to be given by (5.22).

We now turn to the parity odd S-matrix module. Once again the bare and local module each have a single generator, from which it follows immediately that the local module is freely generated. The generator of the local module is proportional to

$$
O_{\mathbf{A}}^{D=6}=s t u \cdot o_{\mathbf{A}}^{D=6}
$$

(see (D.2)) an expression that is dual, in the sense of subsection 4.4, to the Lagrangian ${ }^{85}$

$$
O_{\mathbf{A}}^{D=6}=F^{a b} *\left(\partial_{a} F \wedge \partial_{b} F \wedge F\right) .
$$

As this generator has derivative dimension 6 and - like $o_{\mathbf{A}}^{D=6}$ (see (D.2)) transforms in the $\mathbf{1}_{\mathbf{A}}$ of $S_{3}$, its contribution to the S-matrix partition function precisely agrees with (5.30). We thus have a complete 'module' explanation for the plethystic result (5.35).

The most general parity odd S-matrix in $D=6$ is given by

$$
\begin{aligned}
\mathcal{S}_{\text {odd }}^{D=6}= & \mathcal{F}_{\mathrm{A}}^{D=6}(t, u)\left(8\left(p_{a}^{1} \epsilon_{b}^{1}-p_{b}^{1} \epsilon_{a}^{1}\right) p_{a}^{2} p_{b}^{3} *\left(\epsilon^{2} \wedge k^{2} \wedge \epsilon^{3} \wedge k^{3} \wedge \epsilon^{4} \wedge k^{4}\right)\right. \\
& +8\left(p_{a}^{2} \epsilon_{b}^{2}-p_{b}^{2} \epsilon_{a}^{2}\right) p_{a}^{1} p_{b}^{4} *\left(\epsilon^{1} \wedge k^{1} \wedge \epsilon^{3} \wedge k^{3} \wedge \epsilon^{4} \wedge k^{4}\right) \\
& +8\left(p_{a}^{3} \epsilon_{b}^{3}-p_{b}^{3} \epsilon_{a}^{3}\right) p_{a}^{4} p_{b}^{1} *\left(\epsilon^{2} \wedge k^{2} \wedge \epsilon^{1} \wedge k^{1} \wedge \epsilon^{4} \wedge k^{4}\right) \\
& \left.+8\left(p_{a}^{4} \epsilon_{b}^{4}-p_{b}^{4} \epsilon_{a}^{4}\right) p_{a}^{3} p_{b}^{2} *\left(\epsilon^{2} \wedge k^{2} \wedge \epsilon^{3} \wedge k^{3} \wedge \epsilon^{1} \wedge k^{1}\right)\right)
\end{aligned}
$$

where the function $\mathcal{F}^{O_{\mathbf{A}}^{D=6}}(t, u)$ is a completely antisymmetric function of $s, t$ and $u$. The parity odd Lagrangian from which (5.5) follows takes the form

$$
L_{\text {odd }}^{D=6}=-\sum_{m, n}\left(\mathcal{F}^{O \mathrm{~A}^{D=6}}\right)_{m, n} 2^{m+n}\left(\prod_{i=1}^{m} \prod_{j=1}^{n}\left(\partial_{\nu_{j}} \partial_{\mu_{i}} F_{a b}\left(\partial_{\mu_{i}} \partial_{a} F \wedge \partial_{\nu_{j}} \partial_{b} F \wedge F\right)\right)\right) .
$$

\footnotetext{
${ }^{85}$ While this Lagrangian vanishes as an expression it is non-trivial as the Lagrangian corresponding to the local Module generator, in the sense of subsection 4.4 .
} 
Recall that the generator (5.36) transforms in the $\mathbf{1}_{\mathbf{A}}$ representation. Even though the momentum dependence of the generator is $s t u$ (and so scales like $s^{2}$ in the Regge limit) the first descendant of this generator that is completely symmetric occurs at 12 derivative order (and scales like $s^{5}$ in the Regge limit). None of the parity odd S-matrices in $D=6$ grow like $s^{2}$ or slower in the Regge limit.

\section{$5.6 D=5$}

Remarkably enough the plethystic partition functions in $D=5$ is identical to that for $D \geq 10$. As in $D=6$, the $D=5$ plethystic result has no errors that need correction, so we conclude that the correct $\mathrm{S}$-matrix partition function is given by

$$
I_{\mathrm{v}}^{D=5 \text { even }}=2 x^{4} Z_{\mathbf{3}}(x)+x^{6} Z_{\mathbf{1}_{\mathbf{S}}}(x), \quad I_{\mathrm{v}}^{D=5 \text { odd }}=0 .
$$

Every aspect of (5.39) is easy to understand from our module analysis. The fact that there are no parity odd S-matrices is a consequence of the fact that the parity odd bare module vanishes (see table 3). And the parity even local module of S-matrices is freely generated starting with the generators (5.17). As for $D \geq 8$ most general polynomial $D=4$ S-matrix and corresponding Lagrangian is given by (5.20) and (5.22).

\section{$5.7 \quad D=4$}

For all $D \geq 5$, the number of generators of the local module agreed with the number of generators of the bare module. Also, the condition (2.32) is not satisfied. As a result the local module is also freely generated. In $D=4$, we see a new phenomenon. The number of generators of the local module is more than the number of generators of the bare module. Hence the local generators obey certain relations. This happens both in the parity even as well as parity odd sector. We discuss this case in detail below.

\subsubsection{Parity even}

The rank of the free bare module reduces from 7 to 5 in four dimensions as discussed in appendix $\mathrm{D}$ while the generators of the local module $E_{J}$ in equation (5.17) all remain nonzero. In order to characterize the relations obeyed by $E_{J}$, we focus on the embedding of the local module into bare module (5.19). As discussed in appendix D, in four dimensions, the bare structures $e_{\mathbf{3}, 1}^{D=4,(i)}$ for $i=1,2,3$ become identical. Hence, $e_{\mathbf{3}, 1}^{D=4}$ transforms in $\mathbf{1}_{\mathbf{S}}$ - rather than $\mathbf{3}$ as in higher dimensions (i.e. the $\mathbf{2}_{\mathbf{M}}$ part of $e_{\mathbf{3}, 1}$ trivializes in $D=4$ ). We denote this generator as $e_{\mathbf{3} \rightarrow \mathbf{S}}^{D=4}$. As $E_{\mathbf{S}}^{D=4}$ doesn't have $e_{\mathbf{3}, 1}^{D=4,(i)}$ it is unaffected by this change. The local generators $E_{\mathbf{3}, 1}^{D=4}$ and $E_{\mathbf{3}, 2}^{D=4}$ become,

$$
\begin{aligned}
& E_{\mathbf{3}, 1}^{(1)}=-8 s^{2} e_{\mathbf{3} \rightarrow \mathbf{S}}+8 s^{2} e_{\mathbf{3}, 2}^{(1)}-8 s^{2} e_{\mathbf{S}} \\
& E_{\mathbf{3}, 2}^{(1)}=-2\left(u^{2}+t^{2}\right) e_{\mathbf{3} \rightarrow \mathbf{S}}+2\left(u(s-t) e_{\mathbf{S}, 2}^{(3)}+t(s-u) e_{\mathbf{3}, 2}^{(2)}\right)-2\left(t^{2}+u^{2}\right) e_{\mathbf{S}} .
\end{aligned}
$$

Here and in the rest of the subsection we drop the superscript $D=4$ to avoid clutter (the (2) and (3) components of (5.40) follow by cyclicity as in (5.18)). We have relations in 
the modules generated by $E_{\mathbf{3}, 1}^{(i)}$ and $E_{\mathbf{3}, 2}^{(i)}$ whenever there are non-trivial solutions to the equations

$$
\left(\sum_{i=1,2,3} \frac{1}{2} \mathcal{F}^{E_{\mathbf{3}, 1}^{(i)}}(t, u) E_{\mathbf{3}, 1}^{(i)}\right)+\left(\sum_{i=1,2,3} \frac{1}{2} \mathcal{F}^{E_{\mathbf{3}, 2}^{(i)}}(t, u) E_{\mathbf{3}, 2}^{(i)}\right)=0 .
$$

It is not difficult to see that there are two independent families of solutions,

$$
\begin{aligned}
& \mathcal{F}_{1}^{E_{\mathbf{3}, 1}}(t, u)=\frac{1}{8}\left(s^{2}+t^{2}+u^{2}\right) f(t, u), \\
& \mathcal{F}_{1}^{E_{\mathbf{3}, 2}}(t, u)=t u f(t, u) .
\end{aligned}
$$

and

$$
\begin{aligned}
& \mathcal{F}_{2}^{E_{3,1}}(t, u)=\frac{9}{8} \operatorname{stug}(t, u), \\
& \mathcal{F}_{2}^{E_{3,2}}(t, u)=-\frac{1}{2}\left(2\left(t^{3}+u^{3}\right)-s t u\right) g(t, u) .
\end{aligned}
$$

where $f(t, u)$ and $g(t, u)$ are arbitrary functions that are symmetric in the two arguments.

Completely explicitly, the most general S-matrix is specified a completely symmetric polynomial $\mathcal{F}^{E_{\mathbf{S}}}(t, u)$ along with two variable symmetric polynomials $\mathcal{F}^{E_{\mathbf{3}, 1}}(t, u)$ and $\mathcal{F}^{E_{3,2}}(t, u)$ that are subjected to the equivalence relations

$$
\begin{aligned}
& \mathcal{F}^{E_{\mathbf{3}, 1}}(t, u) \sim \mathcal{F}^{E_{\mathbf{3}, 1}}(t, u)+\frac{9}{8} \operatorname{stug}(t, u)+\frac{1}{8}\left(s^{2}+t^{2}+u^{2}\right) f(t, u), \\
& \mathcal{F}^{E_{\mathbf{3}, 2}}(t, u) \sim \mathcal{F}^{E_{\mathbf{3}, 2}}(t, u)-\frac{1}{2}\left(2\left(t^{3}+u^{3}\right)-s t u\right) g(t, u)+t u f(t, u)
\end{aligned}
$$

where $f(t, u)$ and $g(t, u)$ are arbitrary functions symmetric in $t$ and $u$.

The relation module is thus a rank two free module with one basis element at 8derivative (the first solution in (5.41) - recall that $E_{\mathbf{3}, 1}$ and $E_{\mathbf{3}, 2}$ themselves start at 4 derivative order) and the other at 10-derivative (the second solution in (5.41)). Both generators of the relation module transform in the symmetric representation. Their contribution to the partition function is given by

$$
-\left(x^{8}+x^{10}\right) Z_{\mathbf{1}_{\mathbf{S}}}(x)=-\left(x^{8}+x^{10}\right) \mathrm{D} .
$$

It follows that module considerations lead us to predict that the partition function over parity even S-matrices in $D=4$ is given by

$$
I_{\mathrm{v}}^{D=4 \text { even }}=2 x^{4} Z_{\mathbf{3}}(x)+x^{6} Z_{\mathbf{1}_{\mathbf{S}}}(x)-\left(x^{8}+x^{10}\right) Z_{\mathbf{1}_{\mathbf{S}}}(x)
$$

We will compare the prediction (5.46) against the results of plethystic counting after incorporating contribution of parity odd S-matrices below.

Interestingly, both the relations (5.42) can be thought of as a consequence of a certain 6 derivative quasi-invariant structure reducing from $\mathbf{3}$ to $\mathbf{1}_{\mathbf{S}}$ i.e. vanishing of the mixed representation $\mathbf{2}_{\mathrm{M}}$ at 6 derivative. This quasi-invariant structure is,

$$
\widetilde{E}^{(1)} \equiv \frac{1}{2}\left(u E_{\mathbf{3}, 2}^{(2)}+t E_{\mathbf{3}, 2}^{(3)}\right)+\frac{s}{8}\left(E_{\mathbf{3}, 1}^{(1)}+E_{\mathbf{3}, 1}^{(2)}+E_{\mathbf{3}, 1}^{(3)}\right) .
$$


It is easy to check that $\widetilde{E}^{(1)}=\widetilde{E}^{(2)}=\widetilde{E}^{(3)}$. The first and the second relation (5.41) are simply consequences of

$$
s \widetilde{E}^{(1)}+t \widetilde{E}^{(2)}+u \widetilde{E}^{(3)}=0, \quad\left(s^{2}+2 u t\right) \widetilde{E}^{(1)}+\left(t^{2}+2 u s\right) \widetilde{E}^{(2)}+\left(u^{2}+2 s t\right) \widetilde{E}^{(3)}=0
$$

respectively, which hold true for any symmetric structure.

Note that the most general polynomial S-matrix - and the Lagrangian that generates it - continues to be given by the equations (5.20) and (5.22). The subtlety in this case is that distinct choices of the three functions $\mathcal{F}^{E_{3,1}^{D=4}}(t, u), \mathcal{F}^{E_{\mathbf{3}, 2}^{D=4}}(t, u)$ and $\mathcal{F}^{E_{\mathbf{S}}^{D=4}}(t, u)$ do not all generate distinct S-matrices; choices for these functions that differ by (5.42) yield the same S-matrix (and same corresponding Lagrangian).

\subsubsection{Parity odd}

It is not difficult to verify that the local module of parity odd S-matrices has two sets of generators, one at 4 derivative order and the other at 6 derivative order; in the sense of subsection 4.4 these generators are 'dual' to the Lagrangians

$$
*(F \wedge F) \operatorname{Tr}\left(F^{2}\right), \quad \varepsilon_{\mu \nu \rho \sigma} F^{\mu \nu} \partial^{\rho} F^{a b} \partial^{\sigma} F^{b c} F^{c a} .
$$

The corresponding generators are

$$
\begin{array}{rl}
O_{\mathbf{3}}^{D=4,(1)} \equiv & \left.2 *\left(F_{1} \wedge F_{2}\right) \operatorname{Tr}\left(F_{3} F_{4}\right)\right|_{\mathbb{Z}_{2} \times \mathbb{Z}_{2}}=4 *\left(F_{1} \wedge F_{2}\right) \operatorname{Tr}\left(F_{3} F_{4}\right)+4 *\left(F_{3} \wedge F_{4}\right) \operatorname{Tr}\left(F_{1} F_{2}\right), \\
O_{\mathbf{S}}^{D=4} \equiv & \left.6 \varepsilon_{\mu \nu \rho \sigma} F_{1}^{\mu \nu} \partial^{\rho} F_{2}^{a b} \partial^{\sigma} F_{3}^{b c} F_{4}^{c a}\right|_{\mathbb{Z}_{2} \times \mathbb{Z}_{2}} \\
=6 & 6\left(\varepsilon_{\mu \nu \rho \sigma} F_{1}^{\mu \nu} \partial^{\rho} F_{2}^{a b} \partial^{\sigma} F_{3}^{b c} F_{4}^{c a}+\varepsilon_{\mu \nu \rho \sigma} F_{2}^{\mu \nu} \partial^{\rho} F_{1}^{a b} \partial^{\sigma} F_{4}^{b c} F_{3}^{c a}\right. \\
& \left.\quad+\varepsilon_{\mu \nu \rho \sigma} F_{3}^{\mu \nu} \partial^{\rho} F_{4}^{a b} \partial^{\sigma} F_{1}^{b c} F_{2}^{c a}+\varepsilon_{\mu \nu \rho \sigma} F_{4}^{\mu \nu} \partial^{\rho} F_{3}^{a b} \partial^{\sigma} F_{2}^{b c} F_{1}^{c a}\right) .
\end{array}
$$

While we have not carefully checked that there are no additional generators of the parity odd local module at higher than six derivatives we strongly believe this to be the case (the matching of our final result with plethystic counting can be thought of as extremely non-trivial evidence in favor of this guess).

Here $O_{\mathbf{3}}^{D=4}$ transforms in $\mathbf{3}$ under $S_{3}$ while $O_{\mathbf{S}}^{D=4}$ transforms in $\mathbf{1}_{\mathbf{S}}$. Naively, the contribution of these terms to the partition function would have been

$$
x^{4} Z_{\mathbf{3}}+x^{6} Z_{\mathbf{1}_{\mathbf{S}}}
$$

but just like in the case of parity even structures, $O_{\mathbf{3}}^{D=4}$ 's do not generate the local module freely. This is because the bare module is of rank 2 as described in appendix D. Its generators are

$$
\left.o_{\mathbf{S}, 1}^{D=4} \equiv i N(\widetilde{\varepsilon})_{\mu} \epsilon_{4 \mu}^{\perp} \alpha_{1} \alpha_{2} \alpha_{3}\right|_{\mathbb{Z}_{2} \times \mathbb{Z}_{2},},\left.\quad o_{\mathbf{S}, 2}^{D=4} \equiv i N(\widetilde{\varepsilon})_{\mu} \epsilon_{4 \mu}^{\perp} \epsilon_{1 \nu}^{\perp} \epsilon_{2 \nu}^{\perp} \alpha_{3}\right|_{\mathbb{Z}_{2} \times \mathbb{Z}_{2}}
$$

Note that $o_{\mathbf{S}, 1}^{D=4}$ and $o_{\mathbf{S}, 2}^{D=4}$ both transform in the $\mathbf{1}_{\mathbf{S}}$ representation under $S_{3}$. The embedding of the local module into the bare module is given below. In the rest of the subsection, 
again we will suppress $D=4$ superscript on the local and bare module generators to avoid clutter.

$$
\begin{aligned}
O_{\mathbf{3}}^{(1)} & =8 s^{2}\left(o_{\mathbf{S}, 1}-o_{\mathbf{S}, 2}\right), \quad O_{\mathbf{3}}^{(2)}=8 t^{2}\left(o_{\mathbf{S}, 1}-o_{\mathbf{S}, 2}\right), \quad O_{\mathbf{3}}^{(3)}=8 u^{2}\left(o_{\mathbf{S}, 1}-o_{\mathbf{S}, 2}\right) \\
O_{\mathbf{S}} & =6 s t u\left(o_{\mathbf{S}, 1}-3 o_{\mathbf{S}, 2}\right) .
\end{aligned}
$$

Note that $O_{\mathbf{3}}$ in (5.52) transform in the $\mathbf{3}$ even thought $o_{\mathbf{S}, 1}$ and $o_{\mathbf{S}, 2}$ transform in the $\mathbf{1}_{\mathbf{S}}$ simply because the triplet of functions $\left(s^{2}, t^{2}, u^{2}\right)$ transforms in the $\mathbf{3}$.

As we have mentioned above, $O_{\mathbf{3}}$ and $O_{\mathrm{S}}$ do not generate the local module freely. As every state in the $O_{\mathbf{S}}$ module is proportional to $o_{\mathbf{S}, 1}-3 o_{\mathbf{S}, 2}$ while every state in the $O_{\mathbf{3}}$ module is proportional to $o_{\mathbf{S}, 1}-o_{\mathbf{S}, 2}$, there can be no relations that involve both states generated by $O_{3}$ and states generated by $O_{\mathbf{S}}$; all relations that exist have to work module by module. As stu $\mathcal{F}^{O \mathbf{s}}(t, u)$ vanishes only when $\mathcal{F}^{O \mathbf{s}}(t, u)=0$ it is clear that the module generated by $O_{\mathbf{S}}$ has no relations.

On the other hand the three distinct generators $O_{3}^{(i)}$ are all 'descendants' of the same primary state $o_{\mathbf{S}, 1}-o_{\mathbf{S}, 2}$ so clearly there exist relations between these states. More generally the non-trivial solution to the equations

$$
\sum_{i=1,2,3} \frac{1}{2} \mathcal{F}^{O_{3}^{(i)}}(t, u) O_{\mathbf{3}}^{(i)}=0 .
$$

are given by the two families

$$
\mathcal{F}_{1}^{O_{3}}(t, u)=2 t u f(t, u), \quad \mathcal{F}_{2}^{O_{3}}(t, u)=\left(2\left(t^{3}+u^{3}\right)+(t+u) t u\right) g(t, u) .
$$

where $f(t, u)$ and $g(t, u)$ are arbitrary functions that are symmetric in the two arguments. It follows that the relation module is a rank two free module with one basis element at 8-derivative (first solution in (5.54) ) and the other at 10-derivative (second solution in (5.54)). The generators of the relation module are explicitly given by

$$
\begin{aligned}
& 2 t u O_{\mathbf{3}}^{(1)}+2 u s O_{\mathbf{3}}^{(2)}+2 s t O_{\mathbf{3}}^{(3)} \\
& \left(2\left(t^{3}+u^{3}\right)-s t u\right) O_{\mathbf{3}}^{(1)}+\left(2\left(u^{3}+s^{3}\right)-s t u\right) O_{\mathbf{3}}^{(2)}+\left(2\left(s^{3}+t^{3}\right)-s t u\right) O_{\mathbf{3}}^{(3)} .
\end{aligned}
$$

Both these generators transform in the $\mathbf{1}_{\mathbf{S}}$ representation. The contribution of the relations to the S-matrix partition function is given by

$$
-\left(x^{8}+x^{10}\right) Z_{\mathbf{1}_{\mathbf{S}}}(x)=-\left(x^{8}+x^{10}\right) \mathrm{D} .
$$

Combining (5.50), (5.56) and (5.46), the module prediction for the $D=4$ partition function over S-matrices is given by

$$
\begin{aligned}
I_{\mathrm{v}}^{\text {even }} & =x^{4}\left(2+3 x^{2}+x^{4}-x^{6}\right) \mathrm{D}=2 x^{4} Z_{\mathbf{3}}+x^{6} Z_{\mathbf{1}_{\mathbf{S}}}-\left(x^{8}+x^{10}\right) Z_{\mathbf{1}_{\mathbf{S}}} \\
I_{\mathrm{v}}^{\text {odd }} & =x^{4}\left(1+2 x^{2}-x^{6}\right) \mathrm{D}=x^{4} Z_{\mathbf{3}}+x^{6} Z_{\mathbf{1}_{\mathbf{S}}}-\left(x^{8}+x^{10}\right) Z_{\mathbf{1}_{\mathbf{S}}} .
\end{aligned}
$$

Remarkably enough the prediction (5.7.2) matches exactly with the results of plethystic counting in $D=4$ (see table 6 ). We view this match as an extremely non-trivial check of the completeness of our understanding of photon S-matrices in $D=4$. 
The most general parity four photon parity odd S-matrix is given by

$$
\begin{aligned}
S_{\text {odd }}^{D=4}= & 2 \mathcal{F}^{O_{3}}(t, u)\left(4 *\left(p_{1} \wedge \epsilon_{1} \wedge p_{2} \wedge \epsilon_{2}\left(p_{\mu}^{3} \epsilon_{\nu}^{3}-p_{\nu}^{3} \epsilon_{\mu}^{3}\right)\right)\left(p_{\mu}^{4} \epsilon_{\nu}^{4}-p_{\nu}^{4} \epsilon_{\mu}^{4}\right)+(1 \rightarrow 3,2 \rightarrow 4)\right) \\
& +2 \mathcal{F}^{O_{3}}(u, s)\left(4 *\left(p_{1} \wedge \epsilon_{1} \wedge p_{3} \wedge \epsilon_{3}\left(p_{\mu}^{2} \epsilon_{\nu}^{2}-p_{\nu}^{2} \epsilon_{\mu}^{2}\right)\right)\left(p_{\mu}^{4} \epsilon_{\nu}^{4}-p_{\nu}^{4} \epsilon_{\mu}^{4}\right)+(1 \rightarrow 2,3 \rightarrow 4)\right) \\
& +2 \mathcal{F}^{O_{3}}(s, t)\left(4 *\left(p_{1} \wedge \epsilon_{1} \wedge p_{4} \wedge \epsilon_{4}\left(p_{\mu}^{3} \epsilon_{\nu}^{3}-p_{\nu}^{3} \epsilon_{\mu}^{3}\right)\right)\left(p_{\mu}^{2} \epsilon_{\nu}^{2}-p_{\nu}^{2} \epsilon_{\mu}^{2}\right)+(1 \rightarrow 3,4 \rightarrow 2)\right) \\
& -\mathcal{F}^{O_{\mathbf{S}}}(t, u) \\
& \times\left(2 *\left(p^{2} \wedge p^{3} \wedge p^{1} \wedge \epsilon^{1}\right)\left(p_{c}^{2} \epsilon_{d}^{2}-p_{d}^{2} \epsilon_{c}^{2}\right)\left(p_{d}^{3} \epsilon_{e}^{3}-p_{e}^{3} \epsilon_{d}^{3}\right)\left(p_{e}^{4} \epsilon_{c}^{4}-p_{c}^{4} \epsilon_{e}^{4}\right)\right. \\
& +2 *\left(p^{1} \wedge p^{3} \wedge p^{2} \wedge \epsilon^{2}\right)\left(p_{c}^{1} \epsilon_{d}^{1}-p_{d}^{1} \epsilon_{c}^{1}\right)\left(p_{d}^{3} \epsilon_{e}^{3}-p_{e}^{3} \epsilon_{d}^{3}\right)\left(p_{e}^{4} \epsilon_{c}^{4}-p_{c}^{4} \epsilon_{e}^{4}\right) \\
& +2 *\left(p^{2} \wedge p^{1} \wedge p^{3} \wedge \epsilon^{3}\right)\left(p_{c}^{2} \epsilon_{d}^{2}-p_{d}^{2} \epsilon_{c}^{2}\right)\left(p_{d}^{1} \epsilon_{e}^{1}-p_{e}^{1} \epsilon_{d}^{1}\right)\left(p_{e}^{4} \epsilon_{c}^{4}-p_{c}^{4} \epsilon_{e}^{4}\right) \\
& \left.+2 *\left(p^{2} \wedge p^{3} \wedge p^{4} \wedge \epsilon^{4}\right)\left(p_{c}^{2} \epsilon_{d}^{2}-p_{d}^{2} \epsilon_{c}^{2}\right)\left(p_{d}^{3} \epsilon_{e}^{3}-p_{e}^{3} \epsilon_{d}^{3}\right)\left(p_{e}^{1} \epsilon_{c}^{1}-p_{c}^{1} \epsilon_{e}^{1}\right)\right)
\end{aligned}
$$

where $\mathcal{F}^{O \mathbf{s}}(t, u)$ is a general polynomial that is completely symmetric under $S_{3}$ and $\mathcal{F}^{O_{3}}(t, u)$ is a polynomial that is symmetric in the two arguments and is only defined up to the addition of specific functions $\mathcal{F}_{1}^{O_{3}}(t, u)$ and $\mathcal{F}_{2}^{O_{3}}(t, u)$ given in equation (5.54).

Recall that all the S-matrices discussed in this section are descendants of the two completely symmetric bare module generators $o_{\mathbf{S}, 1}-o_{\mathbf{S}, 2}$ and $o_{\mathbf{S}, 1}-3 o_{\mathbf{S}, 2}$. From the analysis of subsection (2.10) we know that the six descendants of these bare generators that grow no faster than $s^{2}$ in the Regge limit are

$$
\begin{array}{lll}
\left(o_{\mathbf{S}, 1}-o_{\mathbf{S}, 2}\right), & \left(s^{2}+t^{2}+u^{2}\right)\left(o_{\mathbf{S}, 1}-o_{\mathbf{S}, 2}\right), & \text { stu }\left(o_{\mathbf{S}, 1}-o_{\mathbf{S}, 2}\right), \\
\left(o_{\mathbf{S}, 1}-3 o_{\mathbf{S}, 2}\right), & \left(s^{2}+t^{2}+u^{2}\right)\left(o_{\mathbf{S}, 1}-3 o_{\mathbf{S}, 2}\right), & \text { stu }\left(o_{\mathbf{S}, 1}-3 o_{\mathbf{S}, 2}\right)
\end{array}
$$

Of these six structures only $\left(s^{2}+t^{2}+u^{2}\right)\left(o_{\mathbf{S}, 1}-o_{\mathbf{S}, 2}\right)$ and $s t u\left(o_{\mathbf{S}, 1}-3 o_{\mathbf{S}, 2}\right)$ are elements of the local module (see (5.52)). It follows that the only local parity odd photonic S-matrices in $D=4$ that grow no slower than $s^{2}$ in the Regge limit are those of the form (5.57) with $\mathcal{F}^{O_{3}}(t, u)$ and $\mathcal{F}^{O} \mathrm{~s}(t, u)$ both constant.

The functions that appear in (5.57) can be Taylor expanded as in (5.11). The Lagrangians that generate the S-matrices (5.57) are given by

$$
\begin{aligned}
L_{\text {odd }}^{D=4}= & \sum_{m, n}\left(\mathcal{F}^{O_{3}}\right)_{m . n} 2^{m+n}\left(\prod_{i=1}^{m} \prod_{j=1}^{n} \operatorname{Tr} \partial_{\nu_{j}} \partial_{\mu_{i}}(F \wedge F) \operatorname{Tr}\left(\partial_{\mu_{i}} F \partial_{\nu_{j}} F\right)\right) \\
& +\sum_{m, n}\left(\mathcal{F}^{O_{\mathbf{s}}}\right)_{m . n} 2^{m+n}\left(\prod_{i=1}^{m} \prod_{j=1}^{n} \partial_{\nu_{j}} \partial_{\mu_{i}}\left(* F_{a b}\right) \partial_{a} \partial_{\mu_{i}} F_{c d} \partial_{b} \partial_{\nu_{j}} F_{d e} F_{e c}\right),
\end{aligned}
$$

Distinct choices of the two functions and do not all yield inequivalent S-matrices; choices of functions that differ by shifts of the form (5.54) yield the same S-matrix and same effective Lagrangian.

\section{$5.8 D=3$}

\subsubsection{Parity even}

As we have noted in appendix $\mathrm{D}$ the parity even part of the bare module in $D=3$ is freely generated by the single generator $e_{\mathbf{S}}$. The fact that there are no transverse direction to the 
momenta plane - and so no transverse polarizations - ensure that $e_{\mathbf{3}, 1}$ and $e_{\mathbf{3}, 2}$ simply vanish in this dimension.

The generators $E_{\mathbf{3}, 1}^{D=3}, E_{\mathbf{3}, 2}^{D=3}$ and $E_{\mathbf{S}}^{D=3}$ are easily evaluated in terms of $e_{\mathbf{S}}$, the generator of the bare module. Suppressing the $D=3$ superscript, we find

$$
\begin{aligned}
E_{\mathbf{3}, 1}^{(1)} & =-8 s^{2} e_{\mathbf{S}}, & E_{\mathbf{3}, 1}^{(2)}=-8 t^{2} e_{\mathbf{S}}, & E_{\mathbf{3}, 1}^{(3)}=-8 u^{2} e_{\mathbf{S}}, \\
E_{\mathbf{3}, 2}^{(1)}=-2\left(t^{2}+u^{2}\right) e_{\mathbf{S}}, & E_{\mathbf{3}, 2}^{(2)}=-2\left(u^{2}+s^{2}\right) e_{\mathbf{S}}, & & E_{\mathbf{3}, 2}=-2\left(s^{2}+t^{2}\right) e_{\mathbf{S}}, \\
E_{\mathbf{S}} & =-6 \text { stu } e_{\mathbf{S}} . & &
\end{aligned}
$$

Each of the generators transform in the $\mathbf{3}$ representation. It is easy to see that the $\mathbf{1}_{\mathbf{S}}$ part and the $\mathbf{2}_{\mathrm{M}}$ parts of these two generators are both proportional to each other so that the generators $E_{\mathbf{3}, 1}^{(i)}$ and $E_{\mathbf{3}, 2}^{(i)}$ are simply linear combinations of each other. The precise relationship between them is

$$
E_{\mathbf{3}, 2}^{(i)}=-\frac{1}{4} E_{\mathbf{3}, 1}^{(i)}+\frac{\sum_{j=1}^{3} E_{\mathbf{3}, 1}^{(j)}}{4}
$$

For this reason we can simply ignore the generators $E_{\mathbf{3}, 2}$ and work only with $E_{\mathbf{3}, 1}$.

Now it is also easy to see that the singlet $E_{\mathbf{S}}$ is a 'level one' descendant of $E_{\mathbf{3}, 1}$. The precise relationship is

$$
E_{\mathbf{S}}=\frac{1}{4}\left(s E_{\mathbf{3}, 1}^{(1)}+t E_{\mathbf{3}, 1}^{(2)}+u E_{\mathbf{3}, 1}^{(3)}\right)
$$

Consequently, $E_{\mathbf{3}, 1}^{(i)}$ are the only independent local module generators and they transform in the 3. The Lagrangian 'dual' to these generators is simply

$$
\left(\operatorname{Tr} F^{2}\right)^{2} \text {. }
$$

It is completely clear that the three $E_{\mathbf{3}, 1}$ are simply level two descendants of the single generator $e_{\mathbf{S}}$ of the bare module. It follows that the local module is not freely generated but is instead subject to relations. In particular the relations

$$
\sum_{i=1,2,3} \frac{1}{2} \mathcal{F}^{E_{3,1}^{(i)}}(t, u) E_{3,1}^{(i)}=0
$$

hold whenever

$$
\mathcal{F}_{1}^{E_{3,1}}(t, u)=2 t u f(t, u), \quad \mathcal{F}_{2}^{E_{3,1}}(t, u)=\left(2\left(t^{3}+u^{3}\right)+(t+u) t u\right) g(t, u) .
$$

where $f(t, u)$ and $g(t, u)$ are arbitrary functions that are symmetric in the two arguments. The functions $g$ and $f$ parameterize null modules. The generators of these modules are both in the $\mathbf{1}_{\mathbf{S}}$ representation and occur at 10 and 8 derivative order respectively. It follows that their contribution to the partition function over S-matrices is given by

$$
-\left(x^{8}+x^{10}\right) Z_{\mathbf{1}_{\mathbf{S}}}(x)=-\left(x^{8}+x^{10}\right) \mathrm{D}
$$

The full parity even part of the partition function, after taking into account the module relations, is thus given by

$$
I_{\mathrm{v}}^{D=3 \text { even }}=x^{4} Z_{\mathbf{3}}(x)-\left(x^{8}+x^{10}\right) Z_{\mathbf{1}_{\mathbf{S}}}(x)
$$


in agreement with the restriction of the $D=3$ part of the results of table 6 to even powers of $x$.

It is easy to check that

$$
I_{\mathrm{v}}^{D=3 \text { even }}=Z_{\mathbf{1}_{\mathbf{S}}}(x)-1
$$

(5.67) expresses the fact that the restriction to singlets of the module generated by $E_{1}^{(i)}$ is the same as the restriction to singlets of the bare module minus the contribution of the dimension zero generator $e_{3}$ itself.

The most general parity even S-matrix and corresponding Lagrangian are given by $(5.20)$ and $(5.22)$ by setting $\mathcal{F}^{E_{\mathbf{3}, 2}^{D=3}}(t, u)=0$ and $\mathcal{F}^{E_{\mathbf{S}}^{D=3}}(t, u)=0$ and $\mathcal{F}^{E_{\mathbf{3}, 1}^{D=3}}(t, u)$ is a polynomial symmetric in $(t, u)$ subject to the equivalence relation (5.64).

\subsubsection{Parity odd}

The local module of parity odd S-matrices is completely generated by the module elements 'dual' to the 5 derivative Lagrangian

$$
\epsilon_{\alpha \beta \gamma} F^{\alpha \beta} \partial^{\gamma} F_{a b} F_{b c} F_{c a}
$$

The quasi-invariant local generators coming from this Lagrangian transform in representation $\mathbf{2}_{\mathbf{M}}$. They are given by

$$
\begin{aligned}
O_{\mathbf{M}}^{D=3,(1)} & =\frac{1}{3}\left(2 \epsilon^{\alpha \beta \gamma} F_{\alpha \beta}^{1} \partial^{\gamma} F_{a b}^{2} F_{b c}^{3} F_{c a}^{4}-\epsilon^{\alpha \beta \gamma} F_{\alpha \beta}^{1} \partial^{\gamma} F_{a b}^{3} F_{b c}^{4} F_{c a}^{2}-\epsilon^{\alpha \beta \gamma} F_{\alpha \beta}^{1} \partial^{\gamma} F_{a b}^{4} F_{b c}^{2} F_{c a}^{3}\right) \\
O_{\mathbf{M}}^{D=3,(2)} & =\frac{1}{3}\left(2 \epsilon^{\alpha \beta \gamma} F_{\alpha \beta}^{1} \partial^{\gamma} F_{a b}^{3} F_{b c}^{4} F_{c a}^{2}-\epsilon^{\alpha \beta \gamma} F_{\alpha \beta}^{1} \partial^{\gamma} F_{a b}^{4} F_{b c}^{2} F_{c a}^{3}-\epsilon^{\alpha \beta \gamma} F_{\alpha \beta}^{1} \partial^{\gamma} F_{a b}^{2} F_{b c}^{3} F_{c a}^{4}\right) \\
O_{\mathbf{M}}^{D=3,(3)} & =\frac{1}{3}\left(2 \epsilon^{\alpha \beta \gamma} F_{\alpha \beta}^{1} \partial^{\gamma} F_{a b}^{4} F_{b c}^{2} F_{c a}^{3}-\epsilon^{\alpha \beta \gamma} F_{\alpha \beta}^{1} \partial^{\gamma} F_{a b}^{2} F_{b c}^{3} F_{c a}^{4}-\epsilon^{\alpha \beta \gamma} F_{\alpha \beta}^{1} \partial^{\gamma} F_{a b}^{3} F_{b c}^{4} F_{c a}^{2}\right)
\end{aligned}
$$

The generators $O_{\mathrm{M}}^{D=3,(i)}$ are antisymmetric under the swap $1 \leftrightarrow 2,1 \leftrightarrow 3$ and $1 \leftrightarrow 4$ respectively. The fact that they transform in $\mathbf{2}_{\mathbf{M}}$ means that $\sum_{i} O_{\mathbf{M}}^{D=3,(i)}=0$ (this last statement is a direct consequence of the fact that the generators (5.69) are a level one descendant of the bare generator $\epsilon_{a b c} p_{1}^{a} p_{2}^{b} p_{3}^{c}$ and so transform in the $\mathbf{2}_{\mathbf{M}}$ representation, simply because the triplet of functions $s, t, u$ transform in the $\mathbf{2}_{\mathbf{M}}$ representation).

As the parity bare module has a single generator (see appendix D) it follows immediately that the parity odd local module is not freely generated. The explicit expression for the generator of the bare module is

$$
o_{\mathbf{A}}^{D=3}=4 \varepsilon_{a b c} p_{1}^{a} p_{2}^{b} p_{3}^{c} \alpha_{1} \alpha_{2} \alpha_{3} \alpha_{4} .
$$

The generators $O_{\mathbf{M}}^{D=3,(i)}$ above are given simply in terms of $o_{\mathbf{A}}^{D=3}$ by

$$
\left(O_{\mathbf{M}}^{(1)}, O_{\mathbf{M}}^{(2)}, O_{\mathbf{M}}^{(3)}\right)=\left(s o_{\mathbf{A}}, t o_{\mathbf{A}}, u o_{\mathbf{A}}\right)
$$

We have dropped the superscript $D=3$ to avoid clutter. It follows immediately from (5.70) that

$$
(u-t) O_{\mathbf{M}}^{(1)}+(s-u) O_{\mathbf{M}}^{(2)}+(t-s) O_{\mathbf{M}}^{(3)}=0
$$


so that the 1.h.s. of (5.71) is a null state. Notice that our 7 derivative null state transforms in the $\mathbf{1}_{\mathbf{S}}$. It follows that the partition function over parity odd S-matrices is given by

$$
x^{5} Z_{\mathbf{2}_{\mathbf{M}}}(x)-x^{7} Z_{\mathbf{S}}(x)=\left(x^{7}+x^{9}-x^{7}\right) \mathrm{D}=x^{9} \mathrm{D}
$$

to the partition function over S-matrices, in agreement with the restriction of the results of the $D=3$ part of table 6 to odd powers of $x$.

The most general S-matrix coming from (5.68) is given by

$$
\begin{aligned}
S_{\mathrm{odd}}^{D=3}=i & \left(\mathcal { F } ^ { O _ { \mathrm { M } } ^ { D = 3 } } ( t , u ) \left(2 *\left(p_{1} \wedge \epsilon_{1} \wedge p_{2}\right)\left(p_{c}^{2} \epsilon_{d}^{2}-p_{d}^{2} \epsilon_{c}^{2}\right)\left(p_{d}^{3} \epsilon_{e}^{3}-p_{e}^{3} \epsilon_{d}^{3}\right)\left(p_{e}^{4} \epsilon_{c}^{4}-p_{c}^{4} \epsilon_{e}^{4}\right)\right.\right. \\
& +(1 \leftrightarrow 2,3 \leftrightarrow 4)+(1 \leftrightarrow 3,2 \leftrightarrow 4)+(1 \leftrightarrow 4,2 \leftrightarrow 3)) \\
& +\mathcal{F}^{O \mathrm{M}=3}(u, s)\left(2 *\left(p_{1} \wedge \epsilon_{1} \wedge p_{3}\right)\left(p_{c}^{3} \epsilon_{d}^{3}-p_{d}^{3} \epsilon_{c}^{3}\right)\left(p_{d}^{2} \epsilon_{e}^{2}-p_{e}^{2} \epsilon_{d}^{2}\right)\left(p_{e}^{4} \epsilon_{c}^{4}-p_{c}^{4} \epsilon_{e}^{4}\right)\right. \\
& +(1 \leftrightarrow 2,3 \leftrightarrow 4)+(1 \leftrightarrow 3,2 \leftrightarrow 4)+(1 \leftrightarrow 4,2 \leftrightarrow 3)) \\
& +\mathcal{F}^{O \mathrm{M}=3}(s, t)\left(2 *\left(p_{1} \wedge \epsilon_{1} \wedge p_{4}\right)\left(p_{c}^{4} \epsilon_{d}^{4}-p_{d}^{4} \epsilon_{c}^{4}\right)\left(p_{d}^{3} \epsilon_{e}^{3}-p_{e}^{3} \epsilon_{d}^{3}\right)\left(p_{e}^{2} \epsilon_{c}^{2}-p_{c}^{2} \epsilon_{e}^{2}\right)\right. \\
& +(1 \leftrightarrow 2,3 \leftrightarrow 4)+(1 \leftrightarrow 3,2 \leftrightarrow 4)+(1 \leftrightarrow 4,2 \leftrightarrow 3)))
\end{aligned}
$$

As their notation indicates, $\mathcal{F}^{O_{\mathrm{M}}^{D=3}}(t, u)$ is the most general antisymmetric polynomial of its arguments satisfying $\sum_{i} \mathcal{F}^{O_{\mathrm{M}}^{D=3,(i)}}(t, u)=0$. Furthermore, it is subjected to

$$
\mathcal{F}^{O=3}(t, u) \sim \mathcal{F}^{O \mathrm{M}=3}(t, u)+(-t+u) f(t, u),
$$

where $f(t, u)$ is any function antisymmetric in its arguments. The equivalence relation is a consequence of (5.71).

All parity odd S-matrices are descendants of the generator $o_{\mathbf{A}}^{D=3}$ that transforms in the $\mathbf{1}_{\mathbf{A}}$. It follows immediately from subsection 2.10 that there are no S-matrices in the module of $o_{\mathbf{A}}^{D=3}$ that yield S-matrices whose Regge growth is slower than $s^{2}$ (the first S-matrix in this module occurs at 9 derivative order and grows like $s^{4}$ in the Regge limit). The most general descendant Lagrangian that gives rise to S-matrix (5.73) is given by

$$
L_{\text {odd }}^{D=3}=\sum_{m, n}\left(\mathcal{F}^{O{ }_{\mathrm{M}}^{D=3}}\right)_{m, n} 2^{m+n}\left(\prod_{i=1}^{m} \prod_{j=1}^{n} \epsilon_{\alpha \beta \gamma} \partial_{\nu_{j}} \partial_{\mu_{i}} F^{\alpha \beta} \operatorname{Tr}\left(\partial^{\gamma} F \partial_{\mu_{i}} F \partial_{\nu_{j}} F\right)\right) .
$$

\subsection{Tree level 4-photon S-matrix in string theory}

In this section, we apply our technology to tree level 4-photon S-matrix, first in Type I string theory then in Bosonic string theory. We will simply express S-matrix in terms of our local structures $E_{\mathbf{3}, 1}, E_{\mathbf{3}, 2}$ and $E_{\mathbf{S}}$.

Type I superstring. The 4-photon scattering amplitude ([38]) is given by,

$$
\mathcal{S}_{\text {Type I }}=f_{\text {Type I }}(t, u) \sum_{i=1,2,3}\left(E_{\mathbf{3}, 2}^{(i)}-\frac{1}{4} E_{\mathbf{3}, 1}^{(i)}\right) .
$$

where $f_{\text {Type I }}(t, u)$ is specific $S_{3}$ symmetric function. In terms of Lagrangians, this S-matrix is obtained by considering specific derivative contractions on

$$
L_{\text {Type I }} \propto \operatorname{Tr}\left(F^{4}\right)-\frac{1}{4}\left(\operatorname{Tr}\left(F^{2}\right)\right)^{2} .
$$


This specific contraction of field-strength is sometimes called $t_{8} F^{4}$, see for example [39], where $t_{8}$ is a tensor such that

$$
\operatorname{Tr}\left(F^{4}\right)-\frac{1}{4}\left(\operatorname{Tr}\left(F^{2}\right)\right)^{2}=t^{\mu_{1} \ldots \mu_{8}} F_{\mu_{1} \mu_{2}} \ldots F_{\mu_{7}, \mu_{8}} .
$$

Bosonic string. The 4-photon scattering amplitude ([38]) in the case of bosonic strings is given by (5.20), with

$$
\begin{aligned}
& \mathcal{F}^{E_{3,1}}(t, u)=f_{\text {Bosonic }}(t, u)\left(\frac{1}{32}\left(\frac{-(t+u)}{2}+1\right)\left(\frac{t}{2}+1\right)\left(\frac{u}{2}+1\right)\right. \\
& \left.+\frac{1}{256}\left(4 t u+t^{2} u^{2}+2\left(t^{2}+u^{2}\right)\right)\right) \\
& \mathcal{F}^{E_{\mathbf{3}, 2}}(t, u)=f_{\text {Bosonic }}(t, u)\left(\frac{-1}{8}\left(\frac{-(t+u)}{2}+1\right)\left(\frac{t}{2}+1\right)\left(\frac{u}{2}+1\right)+\frac{1}{64}(t+2)(u+2)\right. \\
& \left.\times\left(t^{2}+2 t(u-1)+(u-2) u\right)\right) \\
& \mathcal{F}^{E \mathbf{S}}(t, u)=f_{\text {Bosonic }}(t, u)\left(\frac{1}{96}\left((t+u)^{2}(-(2 t+3))+2(t+u) t^{2}-3 t^{2}+16\right)\right)
\end{aligned}
$$

where $f_{\text {Bosonic }}(t, u)$ is specific $S_{3}$ symmetric function.

\section{Polynomial graviton S-matrices and corresponding Lagrangians}

We now turn to a study of Gravitational S-matrices and corresponding Lagrangians.

\section{1 $D \geq 8$}

As in the case of photon scattering, there are no parity odd gravitational S-matrices for $D \geq 8$. In the rest of this subsubsection we will provide a detailed description of the (automatically parity even) local S-matrix module in $D \geq 8$.

\subsubsection{Modules generated by Lagrangians with 8 or fewer derivatives}

No gravitational Lagrangian that is linear or quadratic in $R_{\mu \nu \alpha \beta}$ produces a polynomial 4 graviton S-matrix (see subsubsection 4.1.3). $G_{\mathbf{S}, 1} \equiv \chi_{6}$ is the unique 3 Riemann Lagrangian that produces a polynomial S-matrix (see (4.20) and (4.25)). All other parity even Lagrangians that generate polynomial S-matrices can be written as the sum of products of derivatives of four Riemann tensors.

The simplest four Riemann Lagrangians are those with eight derivatives. These are constructed from contractions of four Riemann tensors (no derivatives). All inequivalent contractions of four Riemann tensors have been enumerated in [40]. Excluding those structures that involve $R$ and $R_{\mu \nu}$ and so can be removed by field redefinitions (see subsubsection 4.1.3), the authors of [40] find 7 inequivalent contractions in $D \geq 8$.

Five of the seven transform as $\mathbf{3}$ and are labeled as $G_{\mathbf{3}, i}, i=1, \ldots, 5$. One generator transforms as $\boldsymbol{6}_{\text {left }}$, it is labeled as $G_{\mathbf{6}}$. It is convenient to decompose $\mathbf{6}_{\text {left }}$ into $\mathbf{3} \oplus \mathbf{3}_{\mathbf{A}}$. We label these pieces as $G_{\mathbf{3}, 6}$ and $G_{\mathbf{3}_{\mathbf{A}}}$. All these are listed in (6.16) and the associated 
Lagrangians are in (6.17). The remaining 8-derivative generator $G_{\mathbf{3}, 9}$ transforms in the $\mathbf{3}$. The Lagrangian associated to it is,

$$
G_{\mathbf{3}, 9}=R_{p q r s} R_{p t r u} R_{t v q w} R_{u v s w}
$$

Finally there is one additional subtlety that needs to be taken into account. Each of the seven generators $G_{\mathbf{3}, 1} \ldots G_{\mathbf{3}, 6}$ and $G_{\mathbf{3}, 9}$ have a single generator in the $\mathbf{1}_{\mathbf{S}} \cdot{ }^{86}$ One linear combination of these seven $\mathbf{1}_{\mathbf{S}}$ structures is simply the third Lovelock term [39]

$$
\begin{aligned}
\chi_{8} & =\epsilon^{a b c d e f g h} \epsilon^{\alpha \beta \gamma \delta \mu \nu \rho \sigma} R_{a b \alpha \beta} R_{c d \gamma \delta} R_{e f \mu \nu} R_{g h \rho \sigma} \\
& \left.\propto\left(G_{\mathbf{3}, 1}+2 G_{\mathbf{3}, 2}+16 G_{\mathbf{3}, 3}+32 G_{\mathbf{3}, 4}+8 G_{\mathbf{3}, 5}-16 G_{\mathbf{3}, 6}-64 G_{\mathbf{3}, 9}\right)\right|_{\mathbf{s}} .
\end{aligned}
$$

When expanded to fourth order in fluctuations, $\chi_{8}$ and all its 'descendants' simply vanish on-shell. It follows that $\chi_{8}$ corresponds to no module element and plays no role in the discussion that follows. When studying S-matrices, therefore, one of the seven $\mathbf{1}_{\mathbf{S}}$ structures above - lets say the $\mathbf{1}_{\mathbf{S}}$ in $G_{\mathbf{3}, 9}$ - can be re-expressed as a linear combination of the other six, and so is not an independent module generator. As we remove the completely symmetric part from $G_{\mathbf{3}, 9}$, let us relabel it as $G_{\mathbf{2}_{\mathrm{M}}}$ to reflect its correct transformation properties.

In the rest of this subsubsection we focus on the submodule - lets call it $M_{8}$ - of the local gravitational module that is generated by Lagrangians with at most 8 derivatives, i.e. the (independent terms in) descendants of $G_{\mathbf{S}, 1}$ plus $G_{\mathbf{3}, 1} \ldots G_{\mathbf{3}, 6}, G_{\mathbf{3}_{\mathbf{A}}}$ and $G_{\mathbf{2}_{\mathbf{M}}} \cdot{ }^{87}$ It turns out that the submodule of interest to this subsubsection is freely generated (the same holds true for the full local module). This statement - which is simply an unproved assertion at this stage - will effectively be demonstrated later in this subsection by comparison of the 'module' and plethystic partition functions.

Proceeding with the assumption that it is freely generated, we now provide two different but equivalent descriptions of the submodule $M_{8}$. The simpler of the two descriptions of our sub module goes as follows. $G_{\mathbf{S}, 1}$ is clearly a generator of our submodule. The module generated by this 6 derivative element has exactly two 8 derivative 'descendants' which together transform in a single copy of in the $\mathbf{2}_{\mathbf{M}}$. This 8 derivative $\mathbf{2}_{\mathbf{M}}$ is a linear combination of the 8 eight derivative $\mathbf{2}_{\mathrm{M}}$ 's present in the generators dual to the eight derivative generators $G_{\mathbf{3}, 1} \ldots G_{\mathbf{3}, 6}, G_{\mathbf{3}_{\mathrm{A}}}$ and $G_{\mathbf{2}_{\mathrm{M}}}$. The relation can be expressed as follows. Let us define $\left(r^{(1)}, r^{(2)}, r^{(3)}\right)=(s, t, u)$. Then, for example, ${ }^{88}$

${ }^{(i)} G_{\mathbf{S}, 1}=4\left(-G_{\mathbf{3}, 1}^{(i)}-2 G_{\mathbf{3}, 2}^{(i)}-16 G_{\mathbf{3}, 3}^{(i)}+16 G_{\mathbf{3}, 4}^{(i)}-2 G_{\mathbf{3}, 5}^{(i)}+10 G_{\mathbf{3}_{, 6}}^{(i)}+16 G_{\mathbf{2}_{\mathbf{M}}}^{(i)}+\left(4 G_{\mathbf{3}_{\mathbf{A}}}^{(i+1)}-4 G_{\mathbf{3}_{\mathbf{A}}}^{(i+2)}\right)\right)$.

where $(i+1)$ and $(i+2)$ are defined cyclically; - for instance when $i=2,(i+1)=(3)$ and $(i+2)=(1)$.

\footnotetext{
${ }^{86}$ In each case this generator is simply the S-matrix that follows from tree diagrams using the Lagrangians 'dual' the module elements above - i.e. the Lagrangians listed in [40].

${ }^{87}$ In the next subsubsection we will continue to describe the rest of the local module (the part of the module generated by terms with 10 or more derivatives).

${ }^{88}$ Each of $G_{\mathbf{3}, 1} \ldots G_{\mathbf{3}, 5}$ transforms as $\mathbf{1}_{\mathbf{S}}+\mathbf{2}_{\mathrm{M}}, G_{\mathbf{6}}$ transforms as $\mathbf{1}_{\mathbf{S}}+\mathbf{1}_{\mathbf{A}}+2 \cdot \mathbf{2}_{\mathrm{M}}$ and $G_{\mathbf{2}_{\mathrm{M}}}$ transforms as $\mathbf{2}_{\mathrm{M}}$. It follows that there are a total of $8 \mathbf{2}_{\mathrm{M}}$ 's.
} 
This means, the l.h.s. of (6.3) (i.e. the 8 derivative descendant of $G_{\mathbf{S}, 1}$ ) together with the generators $G_{\mathbf{3}, 1} \ldots G_{\mathbf{3}, 6}$ and $G_{\mathbf{3}_{\mathbf{A}}}$ span the space of 8 derivative module elements. Note that $G_{\mathbf{2}_{\mathbf{M}}}$ does not appear in this list of generators; we have (6.2) to eliminate the $\mathbf{1}_{\mathbf{S}}$ part of $G_{\mathbf{3}, 9}$ and have used (6.3) to eliminate the $\mathbf{2}_{\mathrm{M}}$ part of this generator.

As $G_{\mathbf{S}, 1}, G_{\mathbf{3}_{\mathbf{A}}}$ and $G_{\mathbf{3}, 1} \ldots G_{\mathbf{3}, 6}$ are the generators of our (assumed freely generated) submodule. It follows that the partition function over singlets of this submodule is given by

$$
Z(x)=5 x^{8} Z_{\mathbf{3}}(x)+x^{8} Z_{\mathbf{6}}(x)+x^{6} Z_{\mathbf{1}_{\mathbf{S}}}(x) .
$$

While the description of the submodule $M_{8}$ presented above is completely adequate for the purpose of listing and counting S-matrices, it is not sufficient to allow us to find a Lagrangian dual to each of these S-matrices. The reason for this is as follows. While $G_{\mathbf{S}, 1}=\chi_{6}$ itself is a perfectly good Lagrangian, the S-matrices in the module generated by the module element dual to $G_{\mathbf{S}, 1}$ do not have obvious non linearly gauge invariant Lagrangian descriptions. This is because $G_{\mathbf{S}, 1}$ is the product of only 3 Riemann tensors, while in order to build the most general descendant of a generator, we need to be able to act derivatives on four independent objects.

As all 8 derivative terms are given by products of four Riemann tensors, the descendants of all such terms have obvious Lagrangian descriptions. For this reason we will now present a second construction of $M_{8}$ in terms of descendants of only 8 derivative elements. This description allows us to associate a Lagrangian with every element of $M_{8}$.

The second, slightly more complicated description of the submodule $M_{8}$ goes as follows. Let us study a slightly modified submodule - lets call it $M_{8}^{\prime}$ - which is defined to equal the set of all elements in $M_{8}$ that have eight or more derivatives. Since the only element in $M_{8}$ that had fewer than 8 derivatives is only the generator $G_{\mathbf{S}, 1}, M_{8}^{\prime}$ equals $M_{8}$ minus (the module element dual to) $G_{\mathbf{S}, 1}$.

Clearly all 8 derivative Lagrangians, i.e. $G_{\mathbf{3}, 1} \ldots G_{\mathbf{3}_{3,6}}, G_{\mathbf{3}_{\mathrm{A}}}$ and $G_{\mathbf{2}_{\mathrm{M}}}$ are generators of $M_{8}^{\prime}$. Unlike $M_{8}$, however, $M_{8}^{\prime}$ is not freely generated. This follows from the fact that one of the 8 derivative $\mathbf{2}_{\mathbf{M}}$ generators of $M_{8}^{\prime}$ is a descendant of $\chi_{6}$, and so takes the form (see (6.3))

$$
(|s\rangle,|t\rangle,|u\rangle) \equiv\left(s\left|G_{\mathbf{S}, 1}\right\rangle, t\left|G_{\mathbf{S}, 1}\right\rangle, u\left|G_{\mathbf{S}, 1}\right\rangle\right)
$$

As $\left|G_{\mathbf{S}, 1}\right\rangle$ is not a state in our modified submodule, (6.5) is does not express a relationship between two states within the modified submodule. However we can use (6.5) to deduce relations within the submodule as follows. The most general state generated by the triplet (6.5) is

$$
|\chi\rangle=A_{s}|s\rangle+A_{t}|t\rangle+A_{u}|u\rangle
$$

where the otherwise arbitrary functions of $s, t, u, A_{s}, A_{t}$ and $A_{u}$ obey

$$
A_{s}+A_{t}+A_{u}=0
$$

Now the choice

$$
A_{s}=(u-t) \chi, \quad A_{t}=(s-u) \chi, \quad A_{u}=(t-s) \chi
$$

(where $\chi$ is an arbitrary function of $s, t, u$ ) clearly obeys (6.7) and so gives a legitimate descendant of the $\mathbf{2}_{\mathbf{M}}$ (6.6). At the level of (6.6) this descendant is non-trivial. But 
once we substitute (6.5) into (6.6) we immediately find that the state generated by (6.8) vanishes. In other words the vanishing (6.6) with the choice (6.8) is a non-trivial relation within $M_{8}^{\prime}$ (even though this relationship is automatic when viewed within $M_{8}$ ).

As $\chi$ is an arbitrary function, the relations (6.8) form a freely generated relation (or null state) module whose primary (or generator) is (6.6) with $\chi=1$, i.e. is

$$
(u-t)|s\rangle+(s-u)|t\rangle+(t-s)|u\rangle
$$

As the triplets $(|s\rangle,|t\rangle,|u\rangle)$ and $(s, t, u)$ both transform like (2.46) under $S_{3}$, it follows from (2.52) that the 10 derivative state (6.9) transforms in the $\mathbf{1}_{\mathbf{A}}$. It follows that the partition function over the module $M_{8}^{\prime}$ is

$$
Z(x)=5 x^{8} Z_{\mathbf{3}}(x)+x^{8} Z_{\mathbf{6}}(x)+\left(x^{8} Z_{\mathbf{2}_{\mathbf{M}}}(x)-x^{10} Z_{\mathbf{1}_{\mathbf{A}}}(x)\right)
$$

Adding in the contribution of the six derivative state, it then follows that the partition function over $M_{8}$ is

$$
Z(x)=5 x^{8} Z_{\mathbf{3}}(x)+x^{8} Z_{\mathbf{6}}(x)+\left(x^{6}+x^{8} Z_{\mathbf{2}_{\mathbf{M}}}(x)-x^{10} Z_{\mathbf{1}_{\mathbf{A}}}(x)\right)
$$

Using the explicit expressions (2.44) it is easy to verify that

$$
x^{6}+x^{8} Z_{\mathbf{2}_{\mathbf{M}}}(x)-x^{10} Z_{\mathbf{1}_{\mathbf{A}}}(x)=x^{6} Z_{\mathbf{1}_{\mathbf{S}}}(x)
$$

so that (6.10) and (6.4) are actually the same equation.

\subsubsection{The rest of the local submodule}

In the previous subsubsection we have constructed the submodule of the local gravitational module that is generated by 6 and 8 derivative terms. In this subsubsection we will construct the rest of the local module. We proceed by guided guesswork; our guesses will be validated by comparison with plethystic counting.

As we have already accounted for the contribution of $G_{\mathbf{S}, 1}$, all remaining polynomial S-matrices are produced by Lagrangians quartic in the Riemann tensor. In order to capture the contribution of such terms to four graviton S-matrices, it is sufficient to linearize each of the four Riemann tensors and also to work on-shell. On-shell and at linearized order

$$
R_{\mu \nu \rho \sigma} \propto\left(p_{\mu} \epsilon_{\nu}-p_{\nu} \epsilon_{\mu}\right)\left(p_{\rho} \epsilon_{\sigma}-p_{\sigma} \epsilon_{\rho}\right) \propto F_{\mu \nu} F_{\rho \sigma}
$$

Note that the r.h.s. of (6.13) is quadratic in $\epsilon$ as expected. At fixed momentum the Riemann tensor is - formally- the second symmetric power of field strengths

$$
R_{\mu \nu \rho \sigma}(p)=\frac{1}{2} F_{\mu \nu}(p) \otimes F_{\rho \sigma}(p) .
$$

One simple (but not necessarily exhaustive) way to construct polynomial graviton Smatrices is to take the second symmetric tensor power of photon S-matrices. The set of all polynomial gravitational S- matrices that can be constructed in this manner clearly form a submodule of the complete local gravitational module. The symmetric products of 
the three generators of the local photon module ${ }^{89}$ are a special set of states within this submodule. The products of generators may be decomposed into familiar representations of $S_{3}$ as follows

$$
\begin{aligned}
\frac{1}{16} S^{2} E_{\mathbf{3}, 1} & =G_{\mathbf{3}, 1} \oplus G_{\mathbf{3}, 2}, \quad \frac{1}{16} S^{2} E_{\mathbf{3}, 2}=G_{\mathbf{3}, 3} \oplus G_{\mathbf{3}, 4}, \\
\frac{1}{16} E_{(\mathbf{3}, 1} \otimes E_{\mathbf{3}, 2)} & =G_{\mathbf{3}, 5} \oplus G_{\mathbf{6}}=G_{\mathbf{3}, 5} \oplus G_{\mathbf{3}, 6} \oplus G_{\mathbf{3}_{\mathbf{A}}}, \\
\frac{1}{16} E_{(\mathbf{3}, 1} \otimes E_{\mathbf{S})} & =G_{\mathbf{3}, 7}, \quad \frac{1}{16} E_{(\mathbf{3}, 2} \otimes E_{\mathbf{S})}=G_{\mathbf{3}, 8}, \quad \frac{1}{16} S^{2} E_{\mathbf{S}}=G_{\mathbf{S}, 2} .
\end{aligned}
$$

where $S^{2}$ represents the symmetric square of an $S_{3}$ representation. The new (with 10 or higher number of derivatives) generators are $G_{\mathbf{3}, 7}, G_{\mathbf{3}, 8}$ and $G_{\mathbf{S}, 2}$. They are labeled by their $S_{3}$ transformation properties as per the convention.More explicitly the generators so obtained are given by

$$
\begin{aligned}
G_{\mathbf{3}, 1}^{(1)} & \equiv \frac{1}{16} E_{\mathbf{3}, 1}^{(1)} \otimes E_{\mathbf{3}, 1}^{(1)}=R_{a b p q}^{1} R_{a b p q}^{2} R_{c d r s}^{3} R_{c d r s}^{4} \\
G_{\mathbf{3}, 2}^{(1)} & \left.\equiv \frac{1}{16} E_{\mathbf{3}, 1}^{(1)} \otimes E_{\mathbf{3}, 1}^{(2)}\right|_{S}=R_{a b p q}^{1} R_{a b r s}^{3} R_{c d p q}^{4} R_{c d r s}^{2}+R_{a b p q}^{1} R_{a b r s}^{4} R_{c d p q}^{3} R_{c d r s}^{2} \\
G_{\mathbf{3}, 3}^{(1)} & \equiv \frac{1}{16} E_{\mathbf{3}, 2}^{(1)} \otimes E_{\mathbf{3}, 2}^{(1)}=R_{a b p q}^{1} R_{c d r s}^{2} R_{b c q r}^{3} R_{d a s p}^{4} \\
G_{\mathbf{3}, 4}^{(1)} & \left.\equiv \frac{1}{16} E_{\mathbf{3}, 2}^{(1)} \otimes E_{\mathbf{3}, 2}^{(2)}\right|_{S}=R_{a b p q}^{1} R_{c d q r}^{3} R_{b c r s}^{4} R_{d a s p}^{2}+R_{a b p q}^{1} R_{c d q r}^{4} R_{b c r s}^{3} R_{d a s p}^{2} \\
G_{\mathbf{3}, 5}^{(1)} & \equiv \frac{1}{16} E_{\mathbf{3}, 1}^{(1)} \otimes E_{\mathbf{3}, 2}^{(1)}=R_{a b p q}^{1} R_{a b r s}^{2} R_{c d q r}^{3} R_{c d s p}^{4} \\
G_{\mathbf{3}, 6}^{(1)} & \left.\equiv \frac{1}{16} E_{\mathbf{3}, 1}^{(1)} \otimes E_{\mathbf{3}, 2}^{(2)}\right|_{S}=R_{a b p q}^{1} R_{a b q r}^{2} R_{c d r s}^{3} R_{c d s p}^{4}+R_{a b p q}^{1} R_{a b q r}^{2} R_{c d r s}^{4} R_{c d s p}^{3} \\
G_{\mathbf{3}_{\mathbf{A}}}^{(1)} & \left.\equiv \frac{1}{16} E_{\mathbf{3}, 1}^{(1)} \otimes E_{\mathbf{3}, 2}^{(2)}\right|_{A}=R_{a b p q}^{1} R_{a b q r}^{2} R_{c d r s}^{3} R_{c d s p}^{4}-R_{a b p q}^{1} R_{a b q r}^{2} R_{c d r s}^{4} R_{c d s p}^{3} \\
G_{\mathbf{3}, 7}^{(1)} & \left.\equiv \frac{1}{16} E_{\mathbf{3}, 1}^{(1)} \otimes E_{\mathbf{S}}\right|_{\mathbb{Z}_{2} \otimes \mathbb{Z}_{2}}=R_{a b p q}^{1} \partial_{p} R_{a b r s}^{2} \partial_{q} R_{c d s t}^{3} R_{c d t r}^{4} \mid \mathbb{Z}_{2} \otimes \mathbb{Z}_{2} \\
G_{\mathbf{3}, 8}^{(1)} & \left.\equiv \frac{1}{16} E_{\mathbf{3}, 2}^{(1)} \otimes E_{\mathbf{S}}\right|_{\mathbb{Z}_{2} \otimes \mathbb{Z}_{2}}=R_{a b p q}^{1} \partial_{p} R_{c d r s}^{2} \partial_{q} R_{b c s t}^{3} R_{d a t r}^{4} \mid \mathbb{Z}_{2} \otimes \mathbb{Z}_{2} \\
G_{\mathbf{S}, 2} & \equiv \frac{1}{16} E_{\mathbf{S} \mid \mathbb{Z}_{2} \otimes \mathbb{Z}_{2}} \otimes E_{\mathbf{S} \mid \mathbb{Z}_{2} \otimes \mathbb{Z}_{2}}=\left.R_{a b p q}^{1} \partial_{a} \partial_{p} R_{c d r s}^{2} \partial_{b} \partial_{q} R_{d e s t}^{3} R_{e a t r}^{4}\right|_{\mathbb{Z}_{2} \otimes \mathbb{Z}_{2} .} .
\end{aligned}
$$

In (6.16) we have explicitly only listed the (1) components of the generators that transform in $\mathbf{3}$ and in one case in $\mathbf{3}_{\mathbf{A}}$ and their transformation properties are given in (2.49) and (2.50).

\footnotetext{
${ }^{89}$ Recall that the module of parity even polynomial photon S-matrices in $D \geq 5$ was generated by two four derivative generators $E_{1}$ and $E_{2}$ (both of which transform in the $\mathbf{3}$ )and one six derivative generator $E_{3}$ (which transforms in the $\mathbf{1}_{\mathbf{S}}$ ).
} 
The Lagrangians corresponding to all these generators are given by

$$
\begin{aligned}
& G_{\mathbf{3}, 1}=R_{a b p q} R_{b a q p} R_{c d r s} R_{d c s r} \\
& G_{3,2}=R_{\text {pqrs }} R_{\text {pqtu }} R_{\text {tuvw }} R_{\text {rsvw }} \\
& G_{\mathbf{3}, 3}=R_{\text {pqrs }} R_{\text {ptru }} R_{\text {tvuw }} R_{q v s w} \\
& G_{3,4}=-R_{p q r s} R_{p t u w} R_{t v w s} R_{q v r u} \\
& G_{3,5}=R_{p q r s} R_{p q t u} R_{r t v w} R_{\text {suvw }} \\
& G_{\mathbf{6}}=G_{\mathbf{3}, 6} \oplus G_{\mathbf{3}_{\mathrm{A}}}=R_{\text {pqrs }} R_{\text {pqrt }} R_{\text {uvwt }} R_{\text {uvws }} \\
& G_{\mathbf{3}, 7}=R_{p q a b} \partial_{a} R_{q p \mu \nu} \partial_{b} R_{r s \nu \alpha} R_{s r \alpha \mu} \\
& G_{\mathbf{3}, 8}=R_{p q a b} \partial_{a} R_{q r \mu \nu} \partial_{b} R_{r s \nu \alpha} R_{s p \alpha \mu} \\
& G_{\mathbf{S}, 2}=R_{a b p q} \partial_{p} \partial_{a} R_{\mu \nu \beta \gamma} \partial_{q} \partial_{b} R_{\nu \alpha \gamma \delta} R_{\alpha \mu \delta \beta} .
\end{aligned}
$$

In our discussion above we have already encountered the 6 eight derivative module elements, $G_{\mathbf{3}, 1} \ldots G_{\mathbf{3}, 6}$ and $G_{\mathbf{3}_{\mathbf{A}}}$ that arise in the symmetric product $S^{2}\left(E_{\mathbf{3}, 1}+E_{\mathbf{3}, 2}\right)$. Recall that these elements, together with $G_{\mathbf{S}, 1}=\chi_{6}$, were the generators of the submodule $M_{8}$ discussed in subsubsection 6.1.1. The set of seven 8-derivative Lagrangians constructed out of Riemann tensors has also appeared in [5]. Using Mathematica we have verified that this result has the following extension to the full local gravitational module for $D \geq 7$. The set of module elements $G_{\mathbf{S}, 1}, G_{\mathbf{S}, 2}$ and $G_{\mathbf{3}, 1} \ldots G_{\mathbf{3}, 8}$ and $G_{\mathbf{3}_{\mathbf{A}}}$ are all independent generators of the parity even part of the local gravitational module. In other words no one of these objects can be written as a linear sum over descendants of the others.

Note that we have only demonstrated that the module elements listed above are all generators; we have not algebraically demonstrated that there are no other generators of the module. Nonetheless a comparison of the generators we have already identified with the generators of the bare module suggests that this is indeed the case. Note that the generators of the local module identified in the previous paragraph consist of one $\mathbf{1}_{\mathbf{S}}$ at 6 derivative order, five $3 s$ and one 6 at 8 derivative order, two $3 \mathrm{~s}$ at 10 derivative order and one $\mathbf{1}_{\mathbf{S}}$ at 12 derivative order. The number (total of 29) and representation content of this collection of generators precisely matches the number and state content of the bare gravitational module (see table 3 and figure 2).

It will turn out - and we will proceed under the assumption that - the list of generators described above is exhaustive; i.e. that $G_{\mathbf{S}, 1}, G_{\mathbf{S}, 2}$ and $G_{\mathbf{3}, 1} \ldots G_{\mathbf{3}, 8}$ and $G_{\mathbf{3}_{\mathbf{A}}}$ generate the local gravitational module. This statement can be taken to be a guess at this stage, which will be verified below by comparison with explicit results of plethystic counting below. As the number of local generators matches the number of bare generators, it is of importance to know whether the stringent condition (2.32) is obeyed. It turns out it is not. It follows that $G_{\mathbf{S}, 1}, G_{\mathbf{S}, 2}$ and $G_{\mathbf{3}, 1} \ldots G_{\mathbf{3}, 8}$ and $G_{\mathbf{3}_{\mathbf{A}}}$ generate the local gravitational module freely.

\subsubsection{Explicit form of S-matrices and Lagrangians}

We use the notation $I_{\mathrm{t}}^{D}(x)$ to denote the partition function over gravitational S-matrices in $D$ dimensions. Our analysis of the module structure presented above amounts to the 
prediction that

$$
I_{\mathrm{t}}^{D \geq 8, \text { even }}(x)=x^{6} Z_{\mathbf{1}_{\mathbf{S}}}+5 x^{8} Z_{\mathbf{3}}+x^{8} Z_{\mathbf{6}}+2 x^{10} Z_{\mathbf{3}}+x^{12} Z_{\mathbf{1}_{\mathbf{S}}}
$$

The explicit form of the S-matrices (and corresponding local Lagrangians) summed over by (6.18) are, respectively, listed in section K.1 (see (K.38), (K.25), (K.18), (K.10), (K.14), (K.1), (K.6), (K.26) and (K.34)).

\subsubsection{Comparison with plethystic}

As there are no parity odd S-matrices when $D \geq 8$, it is particularly straightforward to compare the parity even module predictions, (6.18), with the results of plethystic counting, table 7 .

Let us first consider the case $D \geq 10$. In this case it is easy to check that the plethystic partition function listed in table 7 exceeds the module prediction (6.18) by $x^{8}-x^{6}$. This difference is easy to understand. The term $-x^{6}$ reflects the fact that the plethystic counting omits to count the Lagrangian $G_{\mathbf{S}, 1}=\chi_{6}$ (recall that the plethystic procedure only counts operators built out of the products of four Riemann letters). On the other hand the extra $x^{8}$ reflects the fact that the plethystic partition function incorrectly counts $\chi_{8}$, defined in (6.2), as a non-trivial Lagrangian term. $\chi_{8}$ does not actually generate a non-trivial Smatrix as its restriction to fourth order in amplitudes is a total derivative. The plethystic procedure does not recognize this fact, as the object that $\chi_{8}$ is a total derivative of is not gauge invariant and so is not itself a quartic polynomial of non-trivial letters.

Let us now turn to $D=9$. In this case the difference between the plethystic result and (6.18) is $-x^{6}+x^{8}-2 x^{9}$ The explanation for the $x^{8}-x^{6}$ is the same as for $D \geq$ 10. The explanation for the additional $-2 x^{9}$ is very similar to the explanation for the difference between plethystic and module predictions for the $D=9$ photon S-matrix (see above (5.24)). In the gravitational case we have two conserved currents (that play the role that $J=* F \wedge F \wedge F \wedge F$ played in the analysis for $D=9$ photons, see above (5.24)). These conserved currents are

$$
*\left(F_{1} \wedge F_{2} \wedge F_{3} \wedge F_{4}\right) \operatorname{Tr}\left(F_{1} F_{2}\right) \operatorname{Tr}\left(F_{3} F_{4}\right), \quad *\left(F_{1} \wedge F_{2} \wedge F_{3} \wedge F_{4}\right) \operatorname{Tr}\left(F_{1} F_{3} F_{2} F_{4}\right) .
$$

As in the photon case, each of these conserved currents contributes $-x^{9}$ to the plethystic counting of singlets. These terms do not correspond to genuine scalar Lagrangians. They represent an error of the plethystic procedure. Once they are removed, the plethystic result reported in table 7 matches perfectly with the correct S-matrix partition function (6.18).

In $D=8$ the plethystic result differs from (6.18) by $-x^{6}+x^{8}+2 x^{8}$. The explanation for $-x^{6}+x^{8}$ is the same as in $D \geq 10$. The explanation for the additional $2 x^{8}$ is, again, similar to the explanation of the excess of $x^{4}$ in the $D=8$ photon plethystic partition function: the plethystic procedure fails to recognize that some Lagrangian terms are total derivatives (and so incorrectly counts them) because they are not total derivatives of objects formed from the products of four gauge invariant letters. In this case the total derivatives that are incorrectly counted by the plethystic procedure are the Lagrangians corresponding to the generators

$$
*\left(F_{1} \wedge F_{2} \wedge F_{3} \wedge F_{4}\right) \operatorname{Tr}\left(F_{1} F_{2}\right) \operatorname{Tr}\left(F_{3} F_{4}\right), \quad *\left(F_{1} \wedge F_{2} \wedge F_{3} \wedge F_{4}\right) \operatorname{Tr}\left(F_{1} F_{3} F_{2} F_{4}\right) .
$$


Explicitly the corresponding Lagrangians are

$$
\begin{aligned}
& \epsilon^{a b c d e f g h} R_{a b \alpha \beta} R_{c d \beta \alpha} R_{e f \gamma \delta} R_{g h \delta \gamma} \sim *(\operatorname{Tr}(R \wedge R) \operatorname{Tr}(R \wedge R)), \\
& \epsilon^{a b c d e f g h} R_{a b \alpha \beta} R_{c d \beta \gamma} R_{e f \gamma \delta} R_{g h \delta \alpha} \sim *(\operatorname{Tr}(R \wedge R \wedge R \wedge R))
\end{aligned}
$$

The Hodge dual of these Lagrangians can be expressed as total derivatives of non-gauge invariant objects [41] (see appendix A)

$$
\begin{aligned}
\operatorname{Tr}(R \wedge R \wedge R \wedge R)=d[ & \operatorname{Tr}(\omega \wedge d \omega \wedge d \omega \wedge d \omega)+\frac{8}{5} \operatorname{Tr}(d \omega \wedge d \omega \wedge \omega \wedge \omega \wedge \omega) \\
& +\frac{4}{5} \operatorname{Tr}(d \omega \wedge \omega \wedge d \omega \wedge \omega \wedge \omega)+\frac{4}{7} \operatorname{Tr}(\omega \wedge \omega \wedge \omega \wedge \omega \wedge \omega \wedge \omega \wedge \omega) \\
& +2 \operatorname{Tr}(d \omega \wedge \omega \wedge \omega \wedge \omega \wedge \omega \wedge \omega)]
\end{aligned}
$$

Similarly,

$$
\operatorname{Tr}(R \wedge R) \operatorname{Tr}(R \wedge R)=d\left[\operatorname{Tr}\left(\omega \wedge d \omega+\frac{2}{3} \omega \wedge \omega \wedge \omega\right) \wedge \operatorname{Tr}(R \wedge R)\right] .
$$

where $\omega_{\mu a b}$ is the spin connection and in notations,

$$
\begin{aligned}
& \operatorname{Tr}(R \wedge R) \operatorname{Tr}(R \wedge R) \sim R_{a}^{b}{ }_{c d} R_{b}{ }^{a}{ }_{e f} R_{\alpha}{ }^{\beta}{ }_{g h} R_{\beta}{ }^{\alpha}{ }_{i j}{ }^{d} x^{c} \wedge d x^{d} \wedge d x^{e} \wedge d x^{f} \wedge d x^{g} \wedge d x^{h} \wedge d x^{i} \wedge d x^{j} \\
& \operatorname{Tr}(R \wedge R \wedge R \wedge R) \sim R_{a}^{b}{ }^{b} d_{b} R_{b f}{ }^{\alpha} R_{\alpha}{ }^{\beta}{ }_{g h} R_{\beta}{ }^{a}{ }_{i j} d x^{c} \wedge d x^{d} \wedge d x^{e} \wedge d x^{f} \wedge d x^{g} \wedge d x^{h} \wedge d x^{i} \wedge d x^{j}
\end{aligned}
$$

Once again the extra $2 x^{8}$ is simply an error of the plethystic procedure; once it is removed the plethystic partition function agrees perfectly with (6.18).

In summary, for all $D \geq 8$ the difference between the plethystic result and (6.18) is entirely a consequence of the fact that the plethystic procedure counts S-matrices only up to minor errors. Once we correct the plethystic result to account for these errors we do indeed recover (6.18), the correct partition function over local S-matrices. The agreement between the (corrected) plethystic result and (6.18) may be viewed as confirmation of the assumption made in 6.1.2, namely that Lagrangians (6.17) plus $\chi_{6}$ are the full set of generators of the local graviton module, and that this module is freely generated.

\section{2 $D=7$}

As far as the parity even terms are concerned the only difference between $D=7$ and $D \geq 8$ is that the expression $\chi_{8}$ defined in (6.2) vanishes identically in $D=7$. From the point of view of enumerating and listing S-matrices and their corresponding Lagrangians this makes absolutely no difference, as, being a total derivative at four graviton order, $\chi_{8}$ did not contribute to four graviton scattering anyway even when it did not identically vanish. It follows, in particular, that (6.18) and all the results of subsubsection (6.1.3) apply unchanged to the parity even part of the $D=7$ S-matrix.

The fact that $\chi_{8}$ vanishes identically in $D=7$ (and so is not counted by the plethystic procedure) does, however, impact the comparison of (6.18) with plethystic, as we see immediately below. 
Even though parity odd S-matrices exist and are counted by the plethystic procedure in $D=7$, it is easy to compare the parity even module prediction (6.18) with the results of plethystic counting reported in table 7 The reason for this is that the plethystic partition function of table 7 is easily decomposed into a part that receives contributions only from the even part of the S-matrix and a part that receives contributions only from the odd part of the S-matrix. The decomposition is as follows: even powers of $x$ in table 7 count parity even structures while odd powers of $x$ count parity odd structures.

The difference between the parity even part of the result reported in table 7 and (6.18) is $-x^{6}$. As above this term reflects the fact that $G_{\mathbf{S}, 1}=\chi_{6}$, which continues to be well defined in $D=7$, is not captured by plethystic counting. ${ }^{90}$

Once we correct the plethystic result (by adding $x^{6}$ to it to account for $\chi_{6}$ ), the even part of the result reported in table 7 agrees perfectly with the correct parity even partition function (6.18).

\subsubsection{Parity odd S-matrices}

We now turn to a study of the parity odd S-matrices in $D=7$. As in subsubsection 6.1.2 we proceed by guided guesswork and then use the results of plethystic counting to validate our guess. The key insight of subsubsection 6.1.2 is that the symmetric square of photon module generators play a special role in the gravitational module. In subsubsection 6.1.2 we only considered the symmetric square of the parity even generators. Extending our considerations to parity odd generators we obtain three parity odd elements of the gravitational module given by

$$
H_{\mathbf{3}, 1}^{D=7}=O_{\mathbf{S}}^{D=7} \otimes E_{\mathbf{3}, 1}, \quad H_{\mathbf{3}, 2}^{D=7}=O_{\mathbf{S}}^{D=7} \otimes E_{\mathbf{3}, 2}, \quad H_{\mathbf{S}}^{D=7}=O_{\mathbf{S}}^{D=7} \otimes E_{\mathbf{S}} .
$$

Recall that $O_{\mathbf{S}}^{D=7}$ is the unique parity odd photon local module generator in $D=7$. It is simply equal to the seven dimensional Chern-Simons term. The first two expressions in (6.24) are of 7th order in derivatives, and transform in the $\mathbf{3}$. The last expression is of 9 th order in derivatives and transforms in the $\mathbf{1}_{\mathbf{S}}$. We have checked that the third expression in (6.24) is not a linear combination of 'descendants' of the first two. Consequently all three expressions in (6.24) are generators of the odd part of the local gravitational module. Note that the number and representation content of the generators (6.24) matches the number and representation content of the odd part of the bare module (see table 3 and appendix D). As in subsubsection 6.1.2 it is thus natural to guess that (6.24) exhausts the space of odd generators in $D=7$. We proceed on this assumption - the correctness of this guess will be demonstrated by the agreement with plethystic. If there are indeed no more generators, it is easy to check that the stringent condition (2.32) is not obeyed so that the odd part of the local module is freely generated. We thus tentatively predict that

$$
I_{\mathrm{t}}^{D=7, \text { odd }}=2 x^{7} Z_{\mathbf{3}}(x)+x^{9} Z_{S}(x)
$$

The odd part of the plethystic partition function reported in table 7 almost agrees with (6.25); the difference between the plethystic result and (6.25) is $-2 x^{7}$ and is easy to

\footnotetext{
${ }^{90}$ The difference is simply $-x^{6}$ rather than $x^{8}-x^{6}$ (as was the case in $D \geq 10$ ) because $\chi_{8}$ is not counted — and so is not over-counted - by the plethystic procedure in these dimensions.
} 
understand. It is a consequence of the fact that the first two generators in (6.24) cannot be written as products of four Riemann tensor letters (even though all their 'descendants' can) and so these expressions are not counted by the plethystic procedure. Once we correct the plethystic partition function by adding in the contribution of these terms, it agrees perfectly with (6.25). This demonstrates that (6.25) is indeed the correct partition function over odd S-matrices, and validates our guess that the expressions in (6.24) are the only odd generators of the $D=7$ module.

The explicit form of the most general parity odd $D=7 \mathrm{~S}$-matrix is given in (K.48).

To end this subsection we explain how the non-linearly gauge invariant Lagrangians that generate (K.48) may be determined. It is not difficult to obtain the Lagrangians dual to the generators (6.24). In order to obtain these quantities we note that working on-shell and to linear order in fluctuations, in the vierbein formulation of general relativity

$$
\left(k_{\mu} \omega_{\nu}(k)-k_{\nu} \omega_{\mu}(k)\right)_{a b} \propto R_{\mu \nu a b}(k) \propto F_{\mu \nu}(k) F_{a b}(k)
$$

Eq. (6.26) can be used to find a linearization of the quantity $\left(\omega_{\mu}\right)_{a b}$. Up to gauge transformations

$$
\left(\omega_{\mu}\right)_{a b}(k)=A_{\mu}(k) F_{a b}(k)
$$

(it is easy to check that (6.27) implies (6.26); at least locally it follows that (6.26) in turn implies that (6.27) holds up to gauge transformations). Using (6.27) and (6.26) it is easy to check that the following linearizations hold

$$
\begin{aligned}
& \operatorname{Tr}(\omega \wedge d \omega+\left.\frac{2}{3} \omega \wedge \omega \wedge \omega\right) \wedge \operatorname{Tr}(R \wedge R) \\
& \propto *\left(A_{1} \wedge F_{2} \wedge F_{3} \wedge F_{4}\right) \operatorname{Tr}\left(F_{1} F_{2}\right) \operatorname{Tr}\left(F_{3} F_{4}\right) \propto H_{3,1}^{D=7} \\
& \operatorname{Tr}(\omega \wedge d \omega \wedge d \omega\wedge d \omega)+\frac{8}{5} \operatorname{Tr}(d \omega \wedge d \omega \wedge \omega \wedge \omega \wedge \omega) \\
&+\frac{4}{5} \operatorname{Tr}(d \omega \wedge \omega\wedge d \omega \wedge \omega \wedge \omega)+\frac{4}{7} \operatorname{Tr}(\omega \wedge \omega \wedge \omega \wedge \omega \wedge \omega \wedge \omega \wedge \omega) \\
&+2 \operatorname{Tr}(d \omega \wedge \omega\wedge \omega \wedge \omega \wedge \omega \wedge \omega) \\
& \propto *\left(A_{1} \wedge F_{2} \wedge F_{3} \wedge F_{4}\right) \operatorname{Tr}\left(F_{1} F_{3} F_{2} F_{4}\right) \propto H_{\mathbf{3}, 2}^{D=7} \\
& \epsilon^{a b c d e f g} R_{a b \alpha \beta} R_{c \alpha \mu \nu} \partial_{\beta} R_{d e \nu \alpha} R_{f g \alpha \mu} \\
& \propto *\left(A_{1} \wedge F_{2} \wedge F_{3} \wedge F_{4}\right) F_{1}^{\alpha \beta} \operatorname{Tr}\left(\partial_{\alpha} F_{3} \partial_{\beta} F_{2} F_{4}\right) \sim H_{\mathbf{S}}^{D=7}
\end{aligned}
$$

Note that the third expression in (6.2.1) is a product of (derivatives of) four Riemann tensors. The Lagrangians that generate the third generator listed in (6.24) are given by contracting indices of the derivatives that act on each of these four Riemann tensors (as in, for instance, the previous subsection).

On the other hand the situation with descendants of the first two (multiplets of) generators of (6.24) is different. As the gauge invariant expressions for the Lagrangians dual to these generators are not given as the products of four gauge invariant 'letters (e.g. four Riemann tensors)', the prescription for obtaining (non-linearly) gauge invariant Lagrangians dual to the descendants of these generators is less clear. In some ways this 'puzzle' is 
similar to that encountered in subsubsection 6.1.1, and the resolution to this puzzle is also similar to that of subsubsection 6.1.1. It is easy to find all the first level (i.e. two derivative) descendants of the first two generators in (6.24). In each case $-H_{\mathbf{3}, 1}^{D=7}$ and $H_{\mathbf{3}, 2}^{D=7}$ - there are 6 such descendants. It is not difficult to recast the explicit expressions for each of these descendants in terms entirely of products of field strengths (i.e. not involving explicit factors of the gauge non invariant $A_{\mu}$ ). It is thus possible to find a non-linearly gauge invariant Lagrangian dual to of each of these two groups of level 1 descendants; the corresponding Lagrangians are quartic polynomials in (derivatives of) $R_{\mu \nu \alpha \beta}$. Consequently, non-linearly gauge invariant Lagrangians that give rise to descendants of these level one descendants i.e. any descendants of level 2 or higher in the original module - are obtained, as usual, by contracting the indices of derivatives on these four Riemann terms in pairs.

That is not, however, the end of the story. In each case the original module transformed in a $\mathbf{3}$ and so had three generators. In each case the set of level one descendants transform in the $2 \cdot \mathbf{2}_{\mathbf{M}}+\mathbf{1}_{\mathbf{S}}+\mathbf{1}_{\mathbf{A}}$ and so are six in number. It follows that the modules generated by these new generators (which were level one descendants of the original generator) is not freely generated but has relations. The generators of these relation modules occur at level two above the generator of the original primary - or level one above the generators of the new module (the analogue of $M_{8}^{\prime}$ in subsection 6.1.1) — and are easily seen to transform in the $\mathbf{1}_{\mathrm{A}}+\mathbf{2}_{\mathrm{M}}$.

In summary, as in subsection 6.1.1 there are two different descriptions of the module generated by each of the first two terms in (6.24). The first simple description is that of a freely generated module generated by the expressions in (6.24). The second consist of the special seven derivative states listed in (6.24) plus the module generated by a 9 derivative primary in the $\boldsymbol{6}_{\text {left }}$ (the new module is the analogue of $M_{8}^{\prime}$ in subsection 6.1.1; its primaries are the level one descendants of the primaries of the original module) minus the states in the relation module which is freely generated by 11 derivative primaries in the $\mathbf{2}_{\mathrm{M}}$ plus the $\mathbf{1}_{\mathbf{A}}$. The fact that these two descriptions are the same is captured by the true partition function identity

$$
x^{7} Z_{\mathbf{3}}(x)=x^{7}+x^{9} Z_{\mathbf{6}_{\text {left }}}(x)-x^{11}\left(Z_{\mathbf{2}_{\mathbf{M}}}(x)+Z_{\mathbf{1}_{\mathbf{A}}}(x)\right)
$$

The second, more complicated, description for the module allows for a straightforward transcriptions to Lagrangians.

While the algorithm for obtaining the (non-linearly gauge invariant) Lagrangians dual to each of the generators of (6.24) is now clear, the final expressions are moderately lengthy, and we do not provide an explicit listing of the corresponding Lagrangians, in the hope that the interested reader would be able to re create these expressions without too much trouble.

\section{$6.3 D=6$}

\subsubsection{Parity even}

In $D=6$ there are two changes in the space of 6 and 8 derivative generators of the even part of the local graviton scattering module (see [40]). The obvious change is that $G_{\mathbf{S}, 1}$ which was nonzero for all $D \geq 7$ - vanishes identically for $D \leq 6$. 
The less obvious change in $D=6$ goes as follows. Recall that at the algebraic level $G_{\mathbf{3}, 1} \ldots G_{\mathbf{3}, 6}, G_{\mathbf{3}_{\mathbf{A}}}$ and $G_{\mathbf{3}, 9}$ were all completely independent for $D \geq 8$. As explained above in $D=7$ (6.2) together with the vanishing of $\chi_{8}$, implied a linear equation between the Lagrangians $G_{\mathbf{3}_{, 1}} \ldots G_{\mathbf{3}_{, 6}}, G_{\mathbf{3}_{\mathbf{A}}}$ and $G_{\mathbf{3}, 9}$, and so between the $\mathbf{1}_{\mathbf{S}}$ components of the generators 'dual' to these Lagrangians in the sense of subsubsection 4.4. In $D \leq 6$ this relationship between the $\mathbf{1}_{\mathbf{S}}$ components of the generators $G_{\mathbf{3}, 1} \ldots G_{\mathbf{3}, 6}, G_{\mathbf{3}_{\mathbf{A}}}$ and $G_{\mathbf{3}, 9}$ is enhanced to a similar relationship between all components of the corresponding generators. In other words in $D \leq 6$ the full 3 generator $G_{3,9}$ is no longer independent of the generators $G_{\mathbf{3}, 1} \ldots G_{\mathbf{3}, 6}, G_{\mathbf{3}_{\mathbf{A}}}$, but can now be written as a sum over the generators $G_{\mathbf{3}, 1} \ldots G_{\mathbf{3}, 6}, G_{\mathbf{3}_{\mathbf{A}}}$ - see (6.3) for a formula. In six dimensions the $\mathbf{2}_{\mathbf{M}}$ part of the generator of $G_{\mathbf{3}, 9}$ can be expressed as $\mathbf{2}_{\mathbf{M}}$ of $G_{\mathbf{3}, 1} \ldots G_{\mathbf{3}, 6}, G_{\mathbf{3}_{\mathbf{A}}}$ due to vanishing of the l.h.s. of (6.3) and the $\mathbf{1}_{\mathbf{S}}$ part was already related to the $\mathbf{1}_{\mathbf{S}}$ of the $G_{\mathbf{3}, 1} \ldots G_{\mathbf{3}, 6}, G_{\mathbf{3}_{\mathbf{A}}}$ due to vanishing of (6.2).

In subsubsection 6.1.1 we had two ways of generating the submodule of the local part of the gravitational module generated by terms with 8 or fewer derivatives. The module $M_{8}$ had $G_{\mathbf{S}, 1}$ as one of its generators and was freely generated. On the other hand the module $M_{8}^{\prime}$ did not include $G_{\mathbf{S}, 1}$ as a generator and consequently was not freely generated. For $D \leq$ $6 M_{8}$ and $M_{8}^{\prime}$ are simply identical as $G_{\mathbf{S}, 1}$ vanishes identically. In $D \geq 7$ the module $M_{8}^{\prime}$ was not freely generated because there was a 'secret' relationship between the 8 derivative $\mathbf{2}_{\mathrm{M}} s$ (see (6.5)). This relationship led to relationships between the descendants of these $\mathbf{2}_{\mathrm{M}} s$. For $D \leq 6$ the fact that $\left|G_{\mathbf{S}, 1}\right\rangle$ vanishes identically turns (6.5) into the simpler equation

$$
(|s\rangle,|t\rangle,|u\rangle)=0
$$

(the l.h.s. is the linear combination of $4 \mathrm{R}$ structures that would have been a descendant of $\chi_{6}$ for $D \geq 7$ ). We can use (6.29) to simply remove $G_{3,9}$ from the list of generators of $M_{8}^{\prime}$. The new module $M_{8}$ is now freely generated by $G_{\mathbf{3}, 1} \ldots G_{\mathbf{3}, 6}, G_{\mathbf{3}_{\mathbf{A}}}$.

The end result of this slightly complicated discussion is simply that we continue to expect the parity even part of the Graviton module to be freely generated in $D=6$, but now with one generator - namely $\chi_{6}$ - removed. This expectation is strengthened by the fact that we see precisely the same reduction in bare structures (table 3 and appendix D). Our (slightly tentative and to be conformed by comparison with plethystic) prediction for the partition function over the parity even part of the $D=6 \mathrm{~S}$-matrix

$$
I_{\mathrm{t}}^{D=6, \text { even }}(x)=5 x^{8} Z_{\mathbf{3}}+x^{8} Z_{\mathbf{6}}+2 x^{10} Z_{\mathbf{3}}+x^{12} Z_{\mathbf{1}}
$$

The most general S-matrix and Lagrangian continue to be given by (K.46) and (K.47) but with the function $\mathcal{F}^{G, 1}(t, u)$ set equal to zero

\subsubsection{Parity odd}

In $D=6$ (and every even dimension) parity odd and parity even S-matrices both contribute terms even in $x$ to the plethystic partition function. The contribution of parity odd and parity even terms to the results of table 7 cannot thus be easily disentangled. For this reason we need to first analyze odd S-matrices before comparing our results with plethystic.

As in subsubsection 6.1.2 we can generate a submodule of the parity odd part of the $D=6$ S-matrix by taking the direct product of the parity even and parity odd parts of the 
local photon S-matrix modules. As in subsubsection 6.1.2 the direct product of generators of these photon modules play a distinguished role in the corresponding product submodule. Recall that the parity odd photon module has a single generator (see (5.37))

$$
O_{\mathbf{A}}^{D=6}=F_{1}^{a b} *\left(\partial_{a} F_{2} \wedge \partial_{b} F_{3} \wedge F_{4}\right) .
$$

As the term in (6.31) is totally anti-symmetric, the tensor products of $E_{\mathbf{3}, 1}$ and $E_{\mathbf{3}, 2}$ with this term transform in the $\mathbf{3}_{\mathbf{A}} \equiv \mathbf{2}_{\mathbf{M}} \oplus \mathbf{1}_{\mathbf{A}}$. The explicit Lagrangians dual to these generators are given by

$$
\begin{aligned}
& H_{\mathbf{3}_{\mathbf{A}}, 1}^{D=6} \equiv O_{\mathbf{A}}^{D=6} \otimes E_{\mathbf{3}, 1}=\epsilon^{a b c d e f} R_{\mu \nu \alpha \beta}^{1} \partial_{\alpha} R_{\nu \mu a b}^{2} \partial_{\beta} R_{\gamma \delta c d}^{3} R_{\delta \gamma e f}^{4} \\
& H_{\mathbf{3}_{\mathbf{A}, 2}, 2}^{D=6} \equiv O_{\mathbf{A}}^{D=6} \otimes E_{\mathbf{3}, 2}=\epsilon^{a b c d e f} R_{\mu \nu \alpha \beta}^{1} \partial_{\alpha} R_{\nu \gamma a b}^{2} \partial_{\beta} R_{\gamma \delta c d}^{3} R_{\delta \mu e f}^{4}
\end{aligned}
$$

In addition to (6.32) there is a third set of 10 derivative generators that transform in the $\mathbf{3}_{\mathrm{A}}$; these generators are given by

$$
H_{\mathbf{3}_{\mathbf{A}, 3}=6}^{D=6}=\epsilon^{a b c d e f} F_{a b}^{1}\left(\partial_{c} F_{\mu \nu}^{2} \partial_{d} F_{\nu \alpha}^{3} F_{\alpha \mu}^{4}\right)\left(F_{\gamma \delta}^{1} F_{e \rho}^{2} F_{f \rho}^{3} F_{\delta \gamma}^{4}\right)
$$

and are generated by the Lagrangian

$$
\epsilon^{a b c d e f} R_{a b \gamma \delta} \partial_{c} R_{\mu \nu e \rho} \partial_{d} R_{\nu \alpha f \rho} R_{\alpha \mu \delta \gamma}
$$

We conjecture that the parity odd part of the local module is freely generated by the three generators $H_{\mathbf{3}_{\mathbf{A}}, 1}, H_{\mathbf{3}_{\mathbf{A}}, 2}$ and $H_{\mathbf{3}_{\mathbf{A}}, 3}$. Note that our guess matches the fact that the bare module in this dimension also consists of 3 copies of the $\mathbf{3}_{\mathbf{A}}$. Note that the Lagrangians in (6.32) and (6.33) themselves vanish as numbers because it they have no $\mathbf{1}_{\mathbf{S}}$ part. However they do not vanish as module generators in the sense of subsection (4.4).

Note that the tensor product of $E_{\mathbf{S}}$ with the generator $O_{\mathbf{A}}^{D=6}$ also yields a parity odd module element. This element transforms in the $\mathbf{1}_{\mathbf{A}}$; it is the completely antisymmetric part of the generator dual to the 12 derivative Lagrangian

$$
\epsilon^{a b c d e f} R_{\mu \nu \alpha \beta} \partial_{\mu} \partial_{\alpha} R_{\delta \rho a b} \partial_{\nu} \partial_{\beta} R_{\rho \gamma c d} R_{\gamma \delta e f} .
$$

Assuming our conjecture is correct (6.35) must be a descendant of (6.34) and (6.32). We have not explicitly checked this; it would be useful to do so.

The module prediction for the partition function over parity odd S-matrices is thus

$$
I_{\mathrm{t}}^{D=6} \text { odd }=3 x^{10} Z_{\mathbf{3}_{\mathrm{A}}}(x)
$$

Remarkably enough the sum of the partition functions (6.36) and (6.30) exactly match the $D=6$ part of the plethystic results reported in table 7 . This agreement is a consistency check for all the guesses in our analysis of both the parity even and parity odd parts of the $D=6$ local module.

The explicit forms of the most general $D=6$ parity odd S-matrices are given in (K.49), and the Lagrangians that generate these S-matrices are listed in (K.50). 


\section{$6.4 D=5$}

\subsubsection{Parity even}

We have noted above that for $D \leq 6$ generator $G_{\mathbf{3}, 9}$ are not independent of $G_{\mathbf{3}, 1} \ldots G_{\mathbf{3}, 6}$, $G_{\mathbf{3}_{\mathrm{A}}}$. In $D=5$ there are further reductions (see [40]). It turns out, in particular, that the five eight derivative Lagrangians $G_{\mathbf{3}, 1} \ldots G_{\mathbf{3}, 5}$ which lead to generators in the $\mathbf{3}$ are no longer independent of each other but obey two linear relations (see (K.53) and (K.54) for details). These relations may be used to remove two of the module elements - say $G_{\mathbf{3}, 3}$ and $G_{\mathbf{3}, 4}$ from the list of generators. Remarkably enough we see a similar reduction in the number and representation content of generators of the bare module in this dimension (see table 3 and appendix D). We thus expect that the module continues to be freely generated (once again this guess is verified by the results of plethystic counting below). In other words we tentatively predict that

$$
I_{\mathrm{t}}^{D=5, \text { even }}(x)=3 x^{8} Z_{\mathbf{3}}+x^{8} Z_{\mathbf{6}}+2 x^{10} Z_{\mathbf{3}}+x^{12} Z_{\mathbf{1}_{\mathbf{S}}}
$$

It is easy to check that (6.37) exactly matches the part of the $D=5$ result reported in table 7 that is even in $x$, confirming the guess that led to (6.37).

The most general parity even part of the S-matrix and Lagrangian continue to be given by (K.46) and (K.47) but with the functions $\mathcal{F}^{G_{3,3}}(t, u)=0$ and $\mathcal{F}^{G_{3,4}}(t, u)=0$ set equal to zero.

\subsubsection{Parity odd}

There were are no parity odd photonic S-matrices in $D=5$; as a consequence no parity odd graviton S-matrices are generated by the direct product of photonic structures. Nonetheless parity odd gravitational S-matrices do exist. We have verified that the lowest (derivative) dimension generator for the parity odd part of the local module is

$$
\begin{aligned}
H_{\mathbf{3}_{\mathbf{A}}}^{D=5} & =\epsilon^{a b c d e} R_{\alpha \beta c d} \partial_{\alpha} \partial_{e} R_{\mu \nu f g} \partial_{\beta} R_{\nu \mu g h} R_{a b h f} \\
& =\epsilon^{a b c d e}\left(F_{\alpha \beta}^{1} \partial_{\alpha} F_{\mu \nu}^{2} \partial_{\beta} F_{\nu \mu}^{3} F_{a b}^{4}\right)\left(F_{c d}^{1} \partial_{e} F_{f g}^{2} F_{g h}^{3} F_{h f}^{4}\right) .
\end{aligned}
$$

This 11 derivative term transforms in $\mathbf{3}_{\mathbf{A}}=\mathbf{2}_{\mathbf{M}} \oplus \mathbf{1}_{\mathbf{A}}$. If (6.38) were to exhaust the set of parity odd generators of the local module, then the number of generators and representation content of the local module would match that of the bare module (see table 3 and appendix D). This leads us to guess that the parity odd part of the local module has no other generators, and that this module is freely generated. This guess yields the following prediction

$$
I_{\mathrm{t}}^{D=5 \text { odd }}=x^{11} Z_{\mathbf{3}_{\mathbf{A}}}(x)
$$

It is easily verified that (6.39) agrees exactly with the part of the $D=5$ result reported in table 7 that is odd in $x$, verifying our guess for the structure of the odd part of the local module. The most general parity odd S-matrix is listed in (K.55) and the corresponding Lagrangian is listed in (K.56). 


\section{5 $D=4$}

\subsubsection{Parity even}

In $D=5$ the independent generators of the parity even part of the local module were $G_{\mathbf{3}, 1}$, $G_{\mathbf{3}, 2}, G_{\mathbf{3}, 5}, G_{\mathbf{3}, 6}, G_{\mathbf{3}, 7}, G_{\mathbf{3}, 8}, G_{\mathbf{3}_{\mathbf{A}}}$ and $G_{\mathbf{S}, 2}$. The list of parity even generators is further reduced in $D=4$ as follows. First, as noted in [40], $G_{\mathbf{6}}=G_{\mathbf{3}_{36}} \oplus G_{\mathbf{3}_{\mathbf{A}}}$ vanishes in $D=4$. Next, as again noted in [40], in $D=4$ there is a new linear relationship between $G_{\mathbf{3}, 1}, G_{\mathbf{3}, 2}$ and $G_{\mathbf{3}, 5}$ (see (K.59) for the exact relation). This relationship can be used to eliminate $G_{\mathbf{3}, 2}$ from our list of generators. Finally, and most subtly, in $D=4$ the 10 derivative generators $G_{\mathbf{3}, 7}$ and $G_{\mathbf{3}, 8}$ both turn out to be 'descendants' of $G_{\mathbf{3}, 1}$ and $G_{\mathbf{3}, 5}$ (i.e. $G_{\mathbf{3}, 7}$ and $G_{\mathbf{3}, 8}$ lie in the module generated by $G_{\mathbf{3}, 1}$ and $G_{\mathbf{3}, 5}$ ) (see (K.61) and (K.60)).

It follows that the parity even part of the S-matrix module is generated by $G_{\mathbf{3}, 1}, G_{\mathbf{3}, 5}$ and $G_{\mathbf{S}, 2}$ (a total of 7 generators). We have verified that there are no further relations between these generators themselves. However in this case the reduction in the number of generators of the bare module is even greater (the bare module has 5 independent structures; one in the $\mathbf{3}$ and two in the $\mathbf{1}_{\mathbf{S}}$ see table 3 and appendix D). As the local module has more generators than the bare module it follows that it is not freely generated. This is not surprising. Recall $G_{\mathbf{3}, 1}^{(1)}=E_{\mathbf{3}, 1}^{(1)} \otimes E_{\mathbf{3}, 1}^{(1)}$ and $G_{\mathbf{3}, 5}^{(1)}=E_{\mathbf{3}, 1}^{(1)} \otimes E_{\mathbf{3}, 2}^{(1)}$ and from the discussion near equation (5.41) we know that $E_{\mathbf{3}, 1}$ and $E_{\mathbf{3}, 2}$ are not independent but obey the relation (5.41). Indeed (5.41) can be used to show that the condition

$$
\sum_{i=1,2,3}\left(\mathcal{F}^{G_{3,1}^{(i)}}(t, u) G_{\mathbf{3}, 1}^{(i)}+\mathcal{F}^{G_{3,5}^{(i)}}(t, u) G_{\mathbf{3}, 5}^{(i)}\right)=0
$$

has two independent families of solution

$$
\begin{array}{ll}
\mathcal{F}_{1}^{G_{3,1}}(t, u)=\left(-s t u-\frac{2}{5}\left(t^{3}+u^{3}\right)\right) f(t, u), & \mathcal{F}_{1}^{G_{3,5}}(t, u)=-4 \mathcal{F}_{1}^{G_{3,1}}(t, u) \\
\mathcal{F}_{2}^{G_{3,1}}(t, u)=\left(2 u^{2} t^{2}\right) g(t, u), & \mathcal{F}_{2}^{G_{3,5}}(t, u)=-4 \mathcal{F}_{2}^{G_{3,1}}(t, u) .
\end{array}
$$

where $g(t, u)$ and $f(t, u)$ are arbitrary functions symmetric in the two arguments. It follows that the relation module has two generators, both in the $\mathbf{1}_{\mathbf{S}}$, the first at 14 derivative order and the second at 16 derivative order. The module prediction for the parity even part of the 4 Graviton S-matrix is thus

$$
I_{\mathrm{t}}^{D=4, \text { even }}(x)=2 x^{8} Z_{\mathbf{3}}+x^{12} Z_{\mathbf{1}_{\mathbf{S}}}-x^{14} Z_{\mathbf{1}_{\mathbf{S}}}-x^{16} Z_{\mathbf{1}_{\mathbf{S}}}
$$


The most general parity invariant 4 graviton S-matrix in 4 dimensions (and corresponding Lagrangian) can now be written down;

$$
\begin{aligned}
S_{T, 4}^{E}=\frac{1}{4} & \left(\mathcal { F } ^ { G _ { 3 , 1 } } ( t , u ) \left[\left(p_{p}^{1} \epsilon_{q}^{1}-p_{q}^{1} \epsilon_{p}^{1}\right)\left(p_{p}^{2} \epsilon_{q}^{2}-p_{q}^{2} \epsilon_{p}^{2}\right)\left(p_{r}^{3} \epsilon_{s}^{3}-p_{s}^{3} \epsilon_{r}^{3}\right)\left(p_{r}^{4} \epsilon_{s}^{4}-p_{s}^{4} \epsilon_{r}^{4}\right)\right.\right. \\
& \left.\times\left(p_{a}^{1} \epsilon_{b}^{1}-p_{b}^{1} \epsilon_{a}^{1}\right)\left(p_{a}^{2} \epsilon_{b}^{2}-p_{b}^{2} \epsilon_{a}^{2}\right)\left(p_{c}^{3} \epsilon_{d}^{3}-p_{d}^{3} \epsilon_{c}^{3}\right)\left(p_{c}^{4} \epsilon_{d}^{4}-p_{d}^{4} \epsilon_{c}^{4}\right)\right] \\
& \left.+\mathcal{F}^{G_{3,1}}(u, s)[3 \leftrightarrow 2]+\mathcal{F}^{G_{3,1}}(s, t)[2 \leftrightarrow 4]\right) \\
+ & \frac{1}{4}\left(\mathcal { F } ^ { G _ { 3 , 5 } } ( t , u ) \left[\left(p_{p}^{1} \epsilon_{q}^{1}-p_{q}^{1} \epsilon_{p}^{1}\right)\left(p_{p}^{2} \epsilon_{q}^{2}-p_{q}^{2} \epsilon_{p}^{2}\right)\left(p_{v}^{3} \epsilon_{w}^{3}-p_{w}^{3} \epsilon_{v}^{3}\right)\left(p_{v}^{4} \epsilon_{w}^{4}-p_{w}^{4} \epsilon_{v}^{4}\right)\right.\right. \\
& \left.\times\left(p_{r}^{1} \epsilon_{s}^{1}-p_{s}^{1} \epsilon_{r}^{1}\right)\left(p_{t}^{2} \epsilon_{u}^{2}-p_{u}^{2} \epsilon_{t}^{2}\right)\left(p_{r}^{3} \epsilon_{t}^{3}-p_{t}^{3} \epsilon_{r}^{3}\right)\left(p_{s}^{4} \epsilon_{u}^{4}-p_{u}^{4} \epsilon_{s}^{4}\right)\right] \\
& \left.+\mathcal{F}^{G_{3}, 5}(u, s)[3 \leftrightarrow 2]+\mathcal{F}^{G_{3}, 5}(s, t)[2 \leftrightarrow 4]\right) \\
+ & \frac{1}{16}\left(\mathcal{F}^{G}, \mathbf{S}_{\mathbf{S}, 2}(t, u)\right) \\
\times & \left(p_{a}^{1} \epsilon_{b}^{1}-p_{b}^{1} \epsilon_{a}^{1}\right) p_{a}^{2}\left(p_{\mu}^{2} \epsilon_{\nu}^{2}-p_{\nu}^{2} \epsilon_{\mu}^{2}\right) p_{b}^{3}\left(p_{\nu}^{3} \epsilon_{\alpha}^{3}-p_{\alpha}^{3} \epsilon_{\nu}^{3}\right)\left(p_{\alpha}^{4} \epsilon_{\mu}^{4}-p_{\mu}^{4} \epsilon_{\alpha}^{4}\right) \\
& \times\left(p_{p}^{1} \epsilon_{q}^{1}-p_{q}^{1} \epsilon_{p}^{1}\right) p_{p}^{2}\left(p_{\beta}^{2} \epsilon_{\gamma}^{2}-p_{\gamma}^{2} \epsilon_{\beta}^{2}\right) p_{q}^{3}\left(p_{\gamma}^{3} \epsilon_{\delta}^{3}-p_{\delta}^{3} \epsilon_{\gamma}^{3}\right)\left(p_{\delta}^{4} \epsilon_{\beta}^{4}-p_{\beta}^{4} \epsilon_{\delta}^{4}\right) \\
& +(1 \leftrightarrow 2)+(1 \leftrightarrow 3)+(1 \leftrightarrow 4)] .
\end{aligned}
$$

The most general descendant Lagrangian which gives rise to this is given by,

$$
\begin{aligned}
L^{4, E}= & \sum_{m, n}\left(\mathcal{F}^{G, 5}\right)_{m, n} 2^{m+n}\left(\prod_{i=1}^{m} \prod_{j=1}^{n}\left(\partial_{\nu_{j}} \partial_{\mu_{i}} R_{\text {pqrs }}\right) R_{p q t u}\left(\partial_{\mu_{i}} R_{r t v w}\right)\left(\partial_{\nu_{i}} R_{\text {suvw }}\right)\right) \\
& +\sum_{m, n}\left(\mathcal{F}^{G_{\mathbf{S}, 2}}\right)_{m, n} 2^{m+n}\left(\prod_{i=1}^{m} \prod_{j=1}^{n}\left(\partial_{\mu_{i}} \partial_{\nu_{j}} R_{a b p q}\right)\left(\partial_{p} \partial_{a} R_{\mu \nu \beta \gamma}\right)\left(\partial_{q} \partial_{b} R_{\nu \alpha \gamma \delta}\right) R_{\alpha \mu \delta \beta}\right) \\
& +\sum_{m, n}\left(\mathcal{F}^{G, 1}\right)_{m, n} 2^{m+n}\left(\prod_{i=1}^{m} \prod_{j=1}^{n}\left(\partial_{\mu_{i}} \partial_{\nu_{j}} R_{a b p q}\right) R_{b a q p}\left(\partial_{\mu_{i}} R_{c d r s}\right)\left(\partial_{\nu_{j}} R_{d c s r}\right)\right) .
\end{aligned}
$$

\subsubsection{Parity odd}

In $D=4$, there are also two parity odd generators for the local module of S-matrices. One of these be thought of as the tensor product $H_{\mathbf{3}}^{D=4,(i)}=O_{\mathbf{3}}^{D=4,(i)} \otimes E_{\mathbf{3}, 1}^{(i)}$. This term transforms in 3 of $S_{3}$. In addition, there is a 12 derivative primary

$$
\begin{aligned}
H_{\mathbf{S}}^{D=4} & =\epsilon^{a b m n} R_{m n g h} \partial_{a} \partial_{g} R_{c d i j} \partial_{b} \partial_{h} R_{d e j k} R_{e c k i} \\
& \left.=\left(* F_{a b}^{1}\right) \operatorname{Tr}\left(\partial_{a} F^{2} \partial_{b} F^{3} F^{4}\right)\right)\left(\left(F_{\alpha \beta}^{1}\right) \operatorname{Tr}\left(\partial_{\alpha} F^{2} \partial_{\beta} F^{3} F^{4}\right)\right)
\end{aligned}
$$

which transforms as $\mathbf{1}_{\mathbf{S}}$. We have 4 local generators while, as discussed in appendix D, the bare module is of rank 2. There must exist relations in this case as well. It turns out that the relations involve only the 3 components of $H_{\mathbf{3}}^{D=4,(i)}$ and are independent of $H_{\mathbf{S}}^{D=4}$. The relations take the form

$$
\left(\sum_{i=1,2,3} \mathcal{F}^{H_{3}^{D=4,(i)}}(t, u) H_{\mathbf{3}}^{D=4,(i)}\right)=0
$$


with two families of solutions

$$
\begin{aligned}
& \mathcal{F}_{1}^{H_{3}^{D=4}}(t, u)=\left(-2\left(t^{3}+u^{3}\right)-s t u\right) f(t, u) \\
& \mathcal{F}_{2}^{H_{3}^{D=4}}(t, u)=\left(-4\left(t^{3} u+u^{3} t\right)+2 t^{2} u^{2}\right) g(t, u) .
\end{aligned}
$$

where $g(t, u)$ and $f(t, u)$ are arbitrary functions symmetric in the two arguments.

The relation module parameterized by the function $f$ is headed by a 14 derivative primary and transforms in the $\mathbf{1}_{\mathbf{S}}$ representation. The relationship module parameterized by the function $g$ is headed by a 16 derivative primary and also transforms in the $\mathbf{1}_{\mathbf{S}}$. It follows that the partition function over the parity even part of S-matrices is given by

$$
I_{\mathrm{t}}^{D=4 \text { odd }}=x^{8} Z_{\mathbf{3}}(x)+x^{12} Z_{\mathbf{1}_{\mathbf{S}}}(x)-x^{14} Z_{\mathbf{1}_{\mathbf{S}}}(x)-x^{16} Z_{\mathbf{1}_{\mathbf{S}}}(x) .
$$

Remarkably enough the sum of the partition functions (6.48) and (6.42) exactly matches the $D=4$ plethystic partition function reported in table 7, verifying the local S-matrix module predicted above. The most general parity odd S-matrix is given by (K.64) and the corresponding Lagrangian is given by (K.63).

\subsection{Regge growth of gravity S-matrices}

In subsection 2.10 we have demonstrated that local S-matrices with 7 or more derivatives all grow faster than $s^{2}$ in the Regge limit. Of all the gravitational S-matrices that appear in the lists provided in this section (in every dimension, and ranging over both parity even and parity odd structures) there is only one that occurs at less than 7 derivative order. This is the S-matrix that follows from $\chi_{6}(\mathrm{~K} .38)$ for $D \geq 7$. This S-matrix is takes the schematic form $s t u \times|b\rangle$ where $|b\rangle$ is a linear combination of the symmetric part of the bare generators listed in lines 1) and 2) of figure 2. This S-matrix grows like $s^{2}$ in the Regge limit.

It follows, in summary, that there are no local gravitational S-matrices that scale like $s^{2}$ or slower for $D \leq 6$. For $D \geq 7$ there is a unique S-matrix of this form, namely the S-matrix (K.38).

\subsection{Tree level 4-graviton S-matrix in string theory}

Einstein gravity. In this section we reproduce the S-matrix coming from Einstein gravity in our basis. The scattering amplitude for Einstein gravity is given by [42],

$$
\begin{aligned}
A_{4 h}^{E G}=\frac{-4 \kappa^{2}}{s t u}( & \frac{1}{2} \epsilon_{2} \cdot \epsilon_{3}\left(s \epsilon_{1} \cdot k_{3} \epsilon_{4} \cdot k_{2}+t \epsilon_{1} \cdot k_{2} \epsilon_{4} \cdot k_{3}\right)+\frac{1}{2} \epsilon_{1} \cdot \epsilon_{4}\left(s \epsilon_{2} \cdot k_{4} \epsilon_{3} \cdot k_{1}+t \epsilon_{2} \cdot k_{1} \epsilon_{3} \cdot k_{4}\right) \\
& +\frac{1}{2} \epsilon_{2} \cdot \epsilon_{4}\left(s \epsilon_{1} \cdot k_{4} \epsilon_{3} \cdot k_{2}+u \epsilon_{1} \cdot k_{2} \epsilon_{3} \cdot k_{4}\right)+\frac{1}{2} \epsilon_{1} \cdot \epsilon_{3}\left(s \epsilon_{2} \cdot k_{3} \epsilon_{4} \cdot k_{1}+u \epsilon_{2} \cdot k_{1} \epsilon_{4} \cdot k_{3}\right) \\
& +\frac{1}{2} \epsilon_{3} \cdot \epsilon_{4}\left(t \epsilon_{1} \cdot k_{4} \epsilon_{2} \cdot k_{3}+u \epsilon_{1} \cdot k_{3} \epsilon_{2} \cdot k_{4}\right)+\frac{1}{2} \epsilon_{1} \cdot \epsilon_{2}\left(t \epsilon_{3} \cdot k_{2} \epsilon_{4} \cdot k_{1}+u \epsilon_{3} \cdot k_{1} \epsilon_{4} \cdot k_{2}\right) \\
& \left.-\frac{1}{4} s t \epsilon_{1} \cdot \epsilon_{4} \epsilon_{2} \cdot \epsilon_{3}-\frac{1}{4} s u \epsilon_{1} \cdot \epsilon_{3} \epsilon_{2} \cdot \epsilon_{4}-\frac{1}{4} t u \epsilon_{1} \cdot \epsilon_{2} \epsilon_{3} \cdot \epsilon_{4}\right)^{2}
\end{aligned}
$$

In terms of notation introduced above,

$$
\mathcal{S}=\left.\frac{-4 \kappa^{2}}{s t u}\left(\frac{1}{32} G_{\mathbf{3}, 1}-\frac{1}{2} G_{\mathbf{3}, 6}+\frac{1}{16} G_{\mathbf{3}, 2}-\frac{1}{4} G_{\mathbf{3}, 5}+G_{\mathbf{3}, 4}+\frac{1}{2} G_{\mathbf{3}, 3}\right)\right|_{\mathbf{1}_{\mathbf{S}}} .
$$


This implies that the following Lagrangian term reproduces the tensor structure of Einstein gravity S-matrix. More precisely $s t u \times A_{4 h}^{E G}$ is reproduced by the following Lagrangian.

$$
\begin{aligned}
\text { stu } L_{4 h}^{E G} \propto & \frac{1}{32}\left(R_{\text {pqrs }} R_{\text {pqrs }}\right)^{2}-\frac{1}{2} R_{\text {pqrs }} R_{\text {pqrt }} R_{\text {uvws }} R_{\text {uvwt }}+\frac{1}{16} R_{p q r s} R_{p q t u} R_{t u v w} R_{r s v w} \\
& -\frac{1}{4} R_{\text {pqrs }} R_{p q t u} R_{r t v w} R_{\text {suvw }}-R_{\text {pqrs }} R_{p t r u} R_{t v w s} R_{q v u w}+\frac{1}{2} R_{p q r s} R_{p t r u} R_{t v u w} R_{q v s w}
\end{aligned}
$$

Sometimes this Lagrangian is written as $t_{16} R^{4}$ [39], where $t_{16}$ is a sixteen index tensor which is the square of eight index tensor $t_{8}$ defined in (5.78).

$$
L_{4 h}^{E G} \propto t^{\mu_{1} \ldots \mu_{8}} t^{\nu_{1} \ldots \nu_{8}} R_{\mu_{1} \mu_{2} \nu_{1} \nu_{2}} \ldots R_{\mu_{7} \mu_{8} \nu_{7} \nu_{8}} .
$$

Type II. The 4-graviton amplitude in Type II superstring theory is given by [38]

$$
A_{4 h}^{s s}=h\left(s, t, u, \alpha^{\prime}\right) A_{4 h}^{E G}
$$

Hence this index structure is reproduced by the same Lagrangian term which reproduces Einstein gravity up to momentum factors.

\section{Exchange contributions}

The most general classical (i.e. tree level) four particle S-matrix that follows from a local Lagrangian is given as the sum of two kinds of terms. These are

- Local S-matrices (i.e. S-matrices that are polynomials in the variables $\epsilon_{i}, k_{i}$ ). These Smatrices, which have their origin in local contact type interactions in the Lagrangian, have been the focus of this paper so far.

- Pole terms that come from the exchange of an intermediate particle.

In the earlier sections of this paper we have performed an exhaustive classification of local S-matrices for four scalar, four photon and four graviton scattering. In this section we will present a preliminary discussion of the pole contributions.

Consider for instance, a four graviton (gggg) scattering amplitude. Consider the pole contribution to this amplitude from the exchange of a particle $P$ of mass $m$ that transforms in the representation $\mathcal{P}$ of the massive Lorentz little group $\mathrm{SO}(D-1)$. The most important thing about this amplitude is that the residue of its pole is completely fixed by the on-shell three particle S-matrix $g g P .{ }^{91}$ It follows that the most general S-matrix that comes from a local Lagrangian is characterized by the masses and spins of the exchange particles $P$

\footnotetext{
${ }^{91}$ Let $s=-\left(k_{1}+k_{2}\right)^{2}$ denote the exchange momentum. The full exchange diagram involves an intermediate off-shell $P$ particle of squared mass $s$ - and so is completely specified only once we are given a 'generalized' three point amplitude in which the gravitons are on-shell but the particle $P$ is off-shell. However all off-shell extensions of the same on-shell amplitude agree when $s=m^{2}$. Moreover these three point amplitudes are polynomial in momenta, so the difference between the numerators exchange diagram built out of any two distinct off-shell extensions of the same on-shell 3 point function contains at least one factor of $\left(s-m^{2}\right)$. This overall propagator cancels the pole originating from the exchange propagator, and we are left with a polynomial S-matrix.
} 
together with the three point $(g g P)$ couplings - in addition to the data that specifies polynomial S-matrices.

In order to complete the classification of polynomial (e.g. 4 graviton) S-matrices presented earlier in this paper into a complete classification of all S-matrices that could possibly originate in local Lagrangians, all we need to do is to work out all possible $g g P$ couplings, and stitch two of these couplings together through the propagator for the particle $P$. Every element in this program is straightforward to carry through. It is easy to list the representations $\mathcal{P}$ of $P$ that can have nonzero on-shell three point functions with our scattering particles. ${ }^{92}$ It is also not difficult to enumerate the most general kinematically allowed on-shell three point functions. The spin $P$ propagator is given simply by the projection - in index space - onto the representation space $\mathcal{P}$ (in the $D-1$ dimensional space orthogonal to $k_{1}+k_{2}$ ) divided by $s-m^{2}$. Sewing these elements together allows us to explicitly construct the most general pole contributions to S-matrices. ${ }^{93}$

In this paper we will not systematically carry through the program outlined in the previous paragraph; we leave this exercise for future work. In this section and appendix L we first present a few sample computations of tree level four point exchange S-matrices. In particular in appendix $\mathrm{L}$ we present

- The most general pole contribution to four scalar scattering.

- One family of photon-photon-spin S exchange contributions to four photon scattering, including, in particular the unique scalar exchange contribution to this process.

- One family of graviton-graviton-spin S exchange contributions to four graviton scattering, including, in particular the unique scalar exchange contribution to this process.

In the main text below we also compute and present results for

- The most general graviton exchange contribution to four graviton scattering.

- The most general massive scalar exchange contribution to four graviton scattering.

- The most general massive spin two exchange contribution to four graviton scattering.

The main focus of our discussion in this section is the Regge growth of exchange contributions to S-matrices. As in the case of contact interactions discussed earlier in this paper, we are particularly interested in classifying those exchange contributions to 4 particle scattering that grow no faster than $s^{2}$ in the Regge limit. ${ }^{94}$ It is very easy to see that the exchange of a massive particle of spin $J^{95}$ in the $t$ channel yields a contribution

\footnotetext{
${ }^{92}$ For instance for scalar $P$ scattering can be nonzero only if $P$ transforms in the traceless symmetric representation with an even number of indices.

${ }^{93}$ When $P$ is itself a graviton there are some minor additional complications, as we will see below.

${ }^{94}$ More generally we would like to classify those exchange contributions which grow no faster than $s^{2}$ in the Regge limit after being combined with suitable polynomial S-matrices of the sort we have enumerated earlier in this paper. Any such combination of an exchange S-matrix plus a 'local counterterm subtraction' reflects an addition to four particle scattering that is not ruled out by the CRG conjecture.

${ }^{95}$ Any massive exchanged particle transforms under some representation of the little group $\operatorname{SO}(D-1)$. There representations can be labelled by Young Tableaux. We say that a particle has spin $J$ if the length of the largest row in the Young Tableaux labelling that particle is $J$.
} 
to scattering that cannot grow faster than $s^{J}{ }^{96}$ Moreover we expect that this inequality is generically saturated - i.e. that spin $J$ exchange in the $t$ channel will grow like $s^{J}$ in the Regge limit (see appendix L for examples). We thus expect that the exchange of spin $J$ particles with $J>2$ will always violate the CRG conjecture; note that this violation is non polynomial in $t$ and so cannot be canceled by a local counterterm. This discussion applies equally well to the scattering of scalars, photons and gravitons.

Let us now turn to the exchange of particles with spin $\leq 2$. The $t$ channel contributions to such exchange processes are always consistent with the CRG conjecture. However the question of whether the $s$ and $u$ channel contributions to exchange contributions violates the CRG conjecture depends on the nature of the external particle. In appendix L we demonstrate that spin zero and spin 2 exchange contributions to four scalar and four photon scattering are both consistent with the CRG conjecture even in the $s$ and $u$ channels. Quite remarkably, however, the explicit computations presented in subsections 7.2 and 7.1 demonstrate that the same is not true for four graviton scattering. In four graviton scattering all possible (non Einstein) exchange of massless gravitons, massive scalars and massive spin 2 particles violate the CRG conjecture in a way that cannot be fixed by a local counterterm.

Why did the sample low spin exchange contributions that we have explicitly computed violate the CRG conjecture for the case of external gravitons? The key point here is that three point $g g P$ S-matrices appear always to be generated by Lagrangian couplings of (derivatives of) two factors of the Riemann tensor to the particle $P$; consequently the three point couplings are always at least 4 derivative order in derivatives. ${ }^{97}$ Assuming this to be the case, in subsection 7.3, we have given an argument that demonstrates that such exchange contributions always violate the CRG conjecture in a way that cannot be canceled by local counterterms, at least in $D \leq 6$. The argument of subsection 7.3 applies to every exchange contribution including those that we have not explicitly computed.

\subsection{4 graviton scattering from graviton exchange}

In this section we construct and study all possible graviton exchange contributions to four point scattering amplitudes of gravitons. As we have explained above, the pole contributions to these exchange diagrams is given by sewing on-shell three point functions through graviton propagators. The kinematically allowed on-shell 3 point functions for gravitons have been listed in (4.22), (4.23), (4.24) (in the exceptional case $d=4$ the amplitude (4.23) is replaced by a parity odd six derivative structure - we will not separately consider this

\footnotetext{
${ }^{96}$ The reason for this is as follows. In the $t$ channel, the scattering particles are grouped into those with momenta $k_{1}, k_{3}$ and those with momenta $k_{2}, k_{4}$. Contraction of momenta within a group - e.g. the dot products $k_{1} \cdot k_{3}$ - produces factor of $t$ but never of $s$. Moreover the unique contraction of momenta between two groups - which happens through the propagator of the exchanged particle - is $\left(k_{1}-k_{3}\right) .\left(k_{2}-k_{4}\right)$. If the exchanged particle has no more than $J$ symmetrized indices, there cannot be more than $J$ factors of $\left(k_{1}-k_{3}\right) .\left(k_{2}-k_{4}\right)$, simply because the original three point function between two scattering particles and the exchanged particle could not have had any vector - in this case $k_{1}-k_{2}$ - contract with more than $J$ indices of the exchanged particle. See appendix L for examples and more details.

${ }^{97}$ The assumption of this section - namely that ggp couplings are always 4 derivative or higher — can, easily be verified by algebraic means - i.e. by simply constructing all gauge invariant $g g P$ three point scattering amplitudes. We leave this straightforward (but potentially lengthy) exercise to future work.
} 
special case). For the convenience of the reader we reproduce the relevant expressions here. ${ }^{98,99}$

$$
\begin{aligned}
A^{R} & =\left(\epsilon_{1} \cdot \epsilon_{2} \epsilon_{3} \cdot p_{1}+\epsilon_{1} \cdot \epsilon_{3} \epsilon_{2} \cdot p_{3}+\epsilon_{2} \cdot \epsilon_{3} \epsilon_{1} \cdot p_{2}\right)^{2} \\
A^{R^{2}} & =\left(\epsilon^{1} \wedge \epsilon^{2} \wedge \epsilon^{3} \wedge k^{1} \wedge k^{2}\right)^{2} \\
A^{R^{3}} & =F_{a b}^{1} F_{a b}^{2} F_{c d}^{2} F_{c d}^{3} F_{e f}^{3} F_{e f}^{1}+\text { perm. }
\end{aligned}
$$

The graviton propagator is simple; it is

$$
G_{\mu \nu, \rho \sigma}=\frac{1}{k^{2}}\left(\frac{1}{2}\left(\eta_{\mu \rho} \eta_{\nu \sigma}+\eta_{\nu \rho} \eta_{\mu \sigma}\right)-\frac{1}{D-2} \eta_{\mu \nu} \eta_{\rho \sigma}\right)
$$

We will now use (7.1) and (7.4) to obtain the graviton exchange contribution for the four point functions in a theory whose (ggg) three point function is given by

$$
A=\alpha_{R} A^{R}+\alpha_{R^{2}} A^{R^{2}}+\alpha_{R^{3}} A^{R^{3}} .
$$

The general exchange S-matrix takes the form

$$
\mathcal{A}=\alpha_{R}^{2} \mathcal{A}_{R-R}+\alpha_{R} \alpha_{R^{2}} \mathcal{A}_{R-R^{2}}+\alpha_{R} \alpha_{R^{3}} \mathcal{A}_{R-R^{3}}+\alpha_{R^{2}}^{2} \mathcal{A}_{R^{2}-R^{2}}+\alpha_{R^{2}} \alpha_{R^{3}} \mathcal{A}_{R^{2}-R^{3}}+\alpha_{R^{3}}^{2} \mathcal{A}_{R^{3}-R^{3}}
$$

Note that the structures $A^{R^{2}}$ and $A^{R^{3}}$ are gauge invariant off-shell (i.e. without using $\left.k_{i}^{2}=0\right)$. It follows that exchange diagrams that sew two of these vertices together i.e. $\mathcal{A}_{R^{2}-R^{2}}, \mathcal{A}_{R^{2}-R^{3}}$ and $\mathcal{A}_{R^{3}-R^{3}}$ - are automatically gauge invariant separately in each channel. In other words these three amplitudes are can be evaluated using the same sewing process utilized when the exchanged particle is not a graviton but another particle. ${ }^{100,101}$

${ }^{98}$ The $R^{2}$ and $R^{3}$ three point functions are sometimes quoted as

$$
A^{R^{2}}=2\left(\epsilon_{1} \cdot \epsilon_{2} \epsilon_{3} \cdot p_{1}+\epsilon_{1} \cdot \epsilon_{3} \epsilon_{2} \cdot p_{3}+\epsilon_{2} . \epsilon_{3} \epsilon_{1} \cdot p_{2}\right)\left(\epsilon_{1} \cdot p_{2} \epsilon_{2} . p_{3} \epsilon_{3} \cdot p_{1}\right)
$$

and

$$
A^{R^{3}}=6\left(\epsilon_{1} \cdot p_{2} \epsilon_{2} \cdot p_{3} \epsilon_{3} \cdot p_{1}\right)^{2}
$$

On-shell these expressions agree with those listed in (7.1). However the form of the expressions in (7.1) has the added advantage that they are off-shell gauge invariant (i.e. gauge invariant without needing to use the conditions $k_{i}^{2}=0$.

${ }^{99}$ As remarked in the footnote of section 4.1 .3 , the Gauss-Bonnet term vanishes in $D=4$ but a new parity odd term appears that contributes to graviton three point function.

${ }^{100}$ In particular each of the amplitudes $\mathcal{A}_{R^{2}-R^{2}}, \mathcal{A}_{R^{2}-R^{3}}$ and $\mathcal{A}_{R^{3}-R^{3}}$ admits a gauge invariant decomposition into a piece that has an $s$ pole, a piece that has a $t$ pole and a piece that has a $u$ pole.

${ }^{101}$ On the other hand exchange pieces that involve one or two $R$ vertices (i.e. terms proportional to one or two powers of $\alpha_{R}$ ) cannot, in general, be decomposed as described above in a gauge invariant manner. This term is best written in the form $\frac{B}{s t u}$ where $B$ is a gauge invariant polynomial. 
The sewing process is easily performed in each channel: we find

$$
\begin{aligned}
& \mathcal{A}_{R^{2}-R^{2}}=\left\{\begin{array}{l}
S^{G_{\mathbf{6}}}+S^{G_{\mathbf{3}, 1}}, \\
\mathcal{F}^{G_{\mathbf{6}}}(t, u)=\frac{1}{8 s}, \mathcal{F}^{G_{\mathbf{3}, 1}}(t, u)=\frac{-D}{32(D-2) s}
\end{array}\right. \\
& \mathcal{A}_{R^{2}-R^{3}}=\left\{\begin{array}{l}
S^{G_{3,6}}+S^{G_{3,5}}+S^{G_{3,1}}+S^{G_{3,2}}+S^{G_{3,7}}+S^{G_{\mathbf{3}_{\mathbf{A}}}}, \\
\mathcal{F}^{G_{3,6}}(t, u)=\frac{3}{8}, \mathcal{F}^{G_{3,5}}(t, u)=\frac{-3}{16}, \mathcal{F}^{G_{\mathbf{3}, 1}}(t, u)=\frac{-3(D+2)}{64(D-2)} \\
\mathcal{F}^{G_{\mathbf{3}, 2}}(t, u)=\frac{3}{32}, \mathcal{F}^{G_{3,7}}(t, u)=\frac{3}{2 s}, \mathcal{F}^{G_{\mathbf{3}} \mathbf{A}}(t, u)=\frac{-12(t-u)}{64 s} .
\end{array}\right. \\
& \mathcal{A}_{R^{3}-R^{3}}=\left\{\begin{array}{l}
S^{G_{\mathbf{3}, 6}}+S^{G_{\mathbf{3}, 5}}+S^{G_{\mathbf{3}, 1}}+S^{G_{\mathbf{3}, 2}}+S^{G_{\mathbf{3}, 7}}+S^{G_{\mathbf{3}} \mathbf{A}}, \\
\mathcal{F}^{G_{\mathbf{3}, 6}}(t, u)=\frac{9 s}{32}, \mathcal{F}^{G_{3,5}}(t, u)=\frac{-9 s}{32}, \mathcal{F}^{G_{3,1}}(t, u)=\frac{-9\left(t^{2}+u^{2}+D u t\right)}{64(D-2) s}, \\
\mathcal{F}^{G_{\mathbf{3}, 2}}(t, u)=\frac{-9 s}{128}, \mathcal{F}^{G_{3,7}}(t, u)=\frac{-9}{4}, \mathcal{F}^{G_{\mathbf{3}} \mathbf{A}}(t, u)=\frac{-9(t-u)}{32} .
\end{array}\right.
\end{aligned}
$$

These S-matrices listed above are formally generated by the non local Lagrangians

$$
\begin{aligned}
& \left(\text { stu) } \mathcal { A } _ { R ^ { 2 } - R ^ { 2 } } \propto \left(-\frac{D}{4(D-2)} \nabla_{\mu} \nabla_{\nu} R_{p q r s} R_{p q r s} \nabla^{\mu} R_{a b c d} \nabla^{\nu} R_{a b c d}\right.\right. \\
& \left.+2 \nabla_{\mu} \nabla_{\nu} R_{p q r s} R_{p q r t} \nabla^{\mu} R_{u v w t} \nabla^{\nu} R_{u v w s}\right) \\
& (s t u) \mathcal{A}_{R^{2}-R^{3}} \propto\left(6 \nabla_{x} \nabla_{y} \nabla_{\mu} R_{p q r s} \nabla^{\mu} R_{p q r t} \nabla^{x} R_{u v w t} \nabla^{y} R_{u v w s}\right. \\
& \text { - } 12 \nabla_{x} \nabla_{y} \nabla_{\mu} R_{\text {pqrs }} R_{\text {pqrt }} \nabla^{x} \nabla^{\mu} R_{u v w t} \nabla^{y} R_{u v w s} \\
& +6 \nabla_{x} \nabla_{y} \nabla^{\mu} R_{\text {pqrs }} R_{\text {pqtu }} \nabla^{x} \nabla_{\mu} R_{\text {rtvw }} \nabla^{y} R_{\text {suvw }} \\
& +\frac{3(D+2)}{2(D-2)} \nabla_{x} \nabla_{y} \nabla^{\mu} R_{\text {pqrs }} R_{\text {pqrs }} \nabla^{x} \nabla_{\mu} R_{\text {pqrs }} \nabla^{y} R_{\text {pqrs }} \\
& +\frac{3}{2} \nabla_{x} \nabla_{y} \nabla^{\mu} R_{p q r s} \nabla_{\mu} R_{p q t u} \nabla^{x} R_{t u v w} \nabla^{y} R_{r s v w} \\
& \left.+12 \nabla_{x} \nabla_{y} R_{\mu \nu a b} \nabla_{a} R_{\nu \mu m n} \nabla_{b} \nabla^{x} R_{\alpha \beta n p} \nabla^{y} R_{\beta \alpha p m}\right) \\
& (s t u) \mathcal{A}_{R^{3}-R^{3}} \propto\left(-36 \nabla^{a} \nabla^{b} \nabla^{\mu} \nabla^{\nu} R_{p q r s} \nabla_{\mu} R_{p q r t} \nabla_{a} \nabla_{\nu} R_{u v w t} \nabla_{b} R_{u v w s}\right. \\
& -18 \nabla^{a} \nabla^{b} \nabla^{\mu} \nabla^{\nu} R_{\text {pqrs }} R_{\text {pqtu }} \nabla_{a} \nabla_{\mu} \nabla_{\nu} R_{\text {rtvw }} \nabla_{b} R_{\text {suvw }} \\
& -18 \nabla^{b} \nabla^{a} \nabla^{\mu} \nabla^{\nu} R_{\text {pqrs }} R_{\text {pqtu }} \nabla_{a} \nabla_{\mu} R_{\text {rtvw }} \nabla_{\nu} \nabla_{b} R_{\text {suvw }} \\
& -\frac{9}{D-2} \nabla^{a} \nabla^{b} \nabla^{\mu} \nabla^{\nu} R_{\alpha \beta c d} R_{\alpha \beta c d} \nabla_{a} R_{p q r s} \nabla_{b} \nabla_{\mu} \nabla_{\nu} R_{p q r s} \\
& -\frac{9 D}{2(D-2)} \nabla^{a} \nabla^{b} \nabla^{\mu} \nabla^{\nu} R_{\alpha \beta c d} R_{\alpha \beta c d} \nabla_{a} \nabla_{\mu} R_{p q r s} \nabla_{\nu} \nabla_{b} R_{p q r s} \\
& +\frac{9}{2} \nabla_{b} \nabla_{a} \nabla^{\mu} \nabla^{\nu} R_{\text {pqrs }} \nabla_{\mu} \nabla_{\nu} R_{\text {pqtu }} \nabla^{b} R_{t u v w} \nabla^{a} R_{\text {rsvw }} \\
& \left.-72 \nabla_{l} \nabla_{k} \nabla_{\gamma} R_{\mu \nu a b} \nabla_{a} R_{\nu \mu m n} \nabla^{\gamma} \nabla_{b} \nabla^{k} R_{\alpha \beta n p} \nabla^{l} R_{\beta \alpha p m}\right)
\end{aligned}
$$


We now turn to the evaluation of the remaining three amplitudes; $\mathcal{A}_{R-R}, \mathcal{A}_{R-R^{2}}$ and $\mathcal{A}_{R-R^{3}}$. These amplitudes are distinguished by the fact that they sew diagrams including at least one copy of the amplitude $A^{R}$, which is gauge invariant on-shell but not off-shell. As the exchange diagram includes an off-shell propagator, the corresponding diagrams are not gauge invariant. Note that this complication is a direct consequence of the fact that the exchanged particle is, itself, a graviton - rather than some completely different particle. This is why the three point functions are not automatically gauge invariant when the exchanged particle is off-shell. It follows that the three exchange diagrams discussed in this paragraph cannot be computed simply by sewing the corresponding three point functions with the graviton propagator. In order to recover gauge invariance we must also add in the contribution of contact 4 point terms from the Einstein action (in the case of $\mathcal{A}_{R-R}$ ), the contribution of the contact term of the Gauss-Bonnet action (in the case of $\mathcal{A}_{R-R^{2}}$ ) and the contribution of the contact term from Riemann cube action (in the case of $\mathcal{A}_{R-R^{3}}$ ). ${ }^{102}$ A direct computation of four graviton tree level scattering matrix starting with the Lagrangian

$$
S=\int \sqrt{g}\left(\alpha_{R} R+\alpha_{R^{2}}\left(R^{2}-4 R_{\mu \nu} R^{\mu \nu}+R_{\mu \nu \rho \sigma} R^{\mu \nu \rho \sigma}\right)+\alpha_{R^{3}} R_{\mu \nu \rho \sigma} R^{\mu \nu a b} R_{a b}{ }^{\rho \sigma}\right),
$$

is algebraically intensive. We use a different method.

We first note that the full gauge invariant result for each of $\mathcal{A}_{R-R}, \mathcal{A}_{R-R^{2}}$ and $\mathcal{A}_{R-R^{3}}$ is given by the sum of a term with an $s$ pole, a term with a $t$ pole, a term with a $u$ pole and a polynomial contact term. Every such S-matrix can be manipulated into the form

$$
\frac{\sum_{i} \beta_{i} S_{i}}{s t u}
$$

Here $S_{i}$ are the most general local gauge invariant S-matrices at 8 derivative order (in the case of $\mathcal{A}_{R-R}$ ), 10 derivative order (in the case of $\mathcal{A}_{R-R^{2}}$ ), and 12 derivative order (in the case of $\mathcal{A}_{R-R^{3}}$ ). Recall that we have already explicitly constructed a basis of all such local S-matrices in section 6 above. $\beta_{i}$ are the as yet unknown constant coefficients of these basis structures.

In order to determine the as yet unknown constants $\beta_{i}$ we now impose the following conditions. The expression (7.12) is a meromorphic function $\epsilon_{i}$ and $k_{i}$. Holding $\epsilon_{i}$ and all other components of $k_{i}$ constant, for a moment, we note that (7.12) has a pole in the variable $s$. We impose the condition that the residue of this pole is the residue of the s channel exchange diagram obtained by sewing the relevant 3 point functions through the graviton propagator (this residue is gauge invariant, because it only samples the 3 point functions when all participating particles are on-shell). This condition unambiguously determines all $\beta_{i}$ coefficients in the case of the amplitudes $\mathcal{A}_{R-R}$ and $\mathcal{A}_{R-R^{2}}$. The fact that these two amplitudes are unambiguously determined by their poles is easy to understand. These amplitudes are, respectively, of homogeneity 2 and 4 in derivatives. An ambiguity in these amplitudes would be a gauge invariant 2 or 4 derivative polynomial S-matrix, and we have demonstrated above that no such S-matrix exists.

\footnotetext{
${ }^{102}$ For example, consider the $R-R^{2}$ exchange which occurs at $\mathcal{O}\left(\alpha_{R} \alpha_{R^{2}}\right)$. There is a polynomial contribution to this exchange diagram from the Gauss bonnet term to fourth order in perturbation. Together the exchange diagram and the contact piece are gauge invariant.
} 
On the other hand the amplitude $\mathcal{A}_{R-R^{3}}$ is of homogeneity 6 in derivatives, and so is determined by its poles only up to the addition of the unique 6 derivative local gauge invariant 4 graviton S-matrix (K.38). Algebraically we do indeed find that $\beta_{i}$ are determined only up to this ambiguity. ${ }^{103}$ In reporting our answer below we make an arbitrary choice to fix this ambiguity. Our final results are

$$
\begin{aligned}
& \mathcal{A}_{R-R}=\left\{\begin{array}{l}
S^{G_{\mathbf{3}, 6}}+S^{G_{\mathbf{3}, 5}}+S^{G_{\mathbf{3}, 3}}+S^{G_{\mathbf{3}, 4}}+S^{G_{\mathbf{3}, 1}}+S^{G_{\mathbf{3}, 2}}, \\
\mathcal{F}^{G_{\mathbf{3}, 6}}(s, t)=\frac{-1}{64 s t u}, \mathcal{F}^{G_{\mathbf{3}, 5}}(t, u)=\frac{-1}{32 s t u}, \mathcal{F}^{G_{\mathbf{3}, 3}}(t, u)=\frac{-1}{32 s t u}, \\
\mathcal{F}^{G_{\mathbf{3}, 4}}(t, u)=\frac{-1}{8 s t u}, \mathcal{F}^{G_{\mathbf{3}, 1}}(t, u)=\frac{1}{256 s t u}, \mathcal{F}^{G_{\mathbf{3}, 2}}(t, u)=\frac{1}{128 s t u},
\end{array}\right. \\
& \mathcal{A}_{R-R^{2}}=\left\{\begin{array}{l}
S^{G_{\mathbf{3}, 6}}+S^{G_{\mathbf{3}, 5}}+S^{G_{\mathbf{3}, 3}}+S^{G_{\mathbf{3}, 4}}+S^{G_{\mathbf{3}, 8}}+S^{G_{\mathbf{3}, 7}}+S^{G_{\mathbf{3}_{\mathbf{A}}}}, \\
\mathcal{F}^{G_{\mathbf{3}, 6}}(s, t)=\frac{1}{32 t u}, \mathcal{F}^{G_{\mathbf{3}, 5}}(t, u)=\frac{-1}{16 t u}, \mathcal{F}^{G_{\mathbf{3}, 3}}(t, u)=\frac{1}{4 t u}, \\
\mathcal{F}^{G_{\mathbf{3}, 4}}(t, u)=\frac{1}{4 t u}, \mathcal{F}^{G_{\mathbf{3}, 8}}(t, u)=\frac{1}{s t u}, \mathcal{F}^{G_{\mathbf{3}, 7}}(t, u)=\frac{-1}{4 s t u}, \mathcal{F}^{G_{\mathbf{3}_{\mathbf{A}}}(t, u)=\frac{-(t-u)}{32 s t u}}
\end{array}\right. \\
& \mathcal{A}_{R-R^{3}}=\left\{\begin{array}{l}
S^{G_{\mathbf{3}, 6}}+S^{G_{\mathbf{3}, 5}}+S^{G_{\mathbf{3}, 3}}+S^{G_{\mathbf{3}, 4}}+S^{G_{\mathbf{3}, 1}}+S^{G_{\mathbf{3}, 2}}+S^{G_{\mathbf{3}, 8}}+S^{G_{\mathbf{S}, 2}}+S^{G_{\mathbf{3}_{\mathbf{A}}},} \\
\mathcal{F}_{\mathbf{3}, 6}(t, u)=\frac{-3 s^{2}+2 t^{2}+2 u^{2}}{12 t_{s t u}}, \mathcal{F}^{G_{\mathbf{3}, 5}}(t, u)=\frac{3}{64 s}, \mathcal{F}^{G_{\mathbf{3}, 3}}(t, u)=\frac{-2 t u-10 s^{2}}{64 s t u}, \\
\mathcal{F}^{G_{\mathbf{3}, 4}}(t, u)=\frac{5}{16 s}, \mathcal{F}^{G_{\mathbf{3}, 1}}(t, u)=\frac{3 t u+s^{2}}{512 s t u}, \mathcal{F}^{G_{\mathbf{3}, 2}}(t, u)=\frac{-t u-s^{2}}{256 s t u}, \mathcal{F}^{G_{\mathbf{3}, 8}}(t, u)=\frac{-1}{t u}, \\
\mathcal{F}^{G_{\mathbf{S}, 2}}(t, u)=\frac{-1}{3 s t u}, \mathcal{F}^{G_{\mathbf{3}} \mathbf{A}}(t, u)=\frac{-s(t-u)}{128 s t u} .
\end{array}\right.
\end{aligned}
$$

The non-local effective Lagrangians that generate these amplitudes are

$$
\begin{aligned}
& (s t u) \mathcal{A}_{R-R} \propto\left(\frac{1}{32}\left(R_{p q r s} R_{p q r s}\right)^{2}-\frac{1}{2} R_{\text {pqrs }} R_{\text {pqrt }} R_{\text {uvws }} R_{\text {uvwt }}+\frac{1}{16} R_{\text {pqrs }} R_{\text {pqtu }} R_{\text {tuvw }} R_{\text {rsvw }}\right. \\
& -\frac{1}{4} R_{\text {pqrs }} R_{p q t u} R_{\text {rtvw }} R_{\text {suvw }}-R_{\text {pqrs }} R_{p t r u} R_{\text {tvws }} R_{\text {qvuw }} \\
& \left.+\frac{1}{2} R_{p q r s} R_{p t r u} R_{t v u w} R_{q v s w}\right) \\
& (s t u) \mathcal{A}_{R-R^{2}} \propto\left(-2\left(\nabla_{\mu} R_{\text {pqrs }} R_{\text {pqrt }} \nabla^{\mu} R_{\text {uvwt }} R_{\text {uvws }}\right)+2\left(\nabla_{\mu} R_{\text {pqrs }} R_{\text {pqtu }} \nabla^{\mu} R_{r t v w} R_{\text {suvw }}\right)\right. \\
& -8\left(\nabla_{\mu} R_{p q r s} \nabla^{\mu} R_{p t r u} R_{t v u w} R_{q v s w}\right)-8\left(\nabla_{\mu} R_{p q r s} \nabla^{\mu} R_{p t u w} R_{t v w s} R_{q v r u}\right) \\
& \left.-8 R_{\alpha \beta a b} \nabla_{a} R_{\beta \gamma c d} \nabla_{b} R_{\gamma \delta d e} R_{\delta \alpha e c}+2 R_{\alpha \beta a b} \nabla_{a} R_{\beta \alpha c d} \nabla_{b} R_{\gamma \delta d e} R_{\delta \gamma e c}\right) \\
& (\text { stu }) \mathcal{A}_{R-R^{3}} \propto\left(\left(-\nabla^{\mu} \nabla^{\nu} R_{\text {pqrs }} \nabla_{\mu} \nabla_{\nu} R_{\text {pqrt }} R_{\text {uvwt }} R_{\text {uvws }}\right.\right. \\
& +2 \nabla^{\mu} \nabla^{\nu} R_{\text {pqrs }} R_{\text {pqrt }} \nabla_{\mu} \nabla_{\nu} R_{\text {uvwt }} R_{\text {uvws }} \\
& \left.+\nabla^{\mu} \nabla^{\nu} R_{\text {pqrs }} \nabla_{\nu} R_{\text {pqrt }} \nabla_{\mu} R_{\text {uvwt }} R_{\text {uvws }}\right)
\end{aligned}
$$

\footnotetext{
${ }^{103}$ The fact that $\beta_{i}$ are determined only up to this ambiguity is very natural from a Lagrangian viewpoint. While the Lagrangians that gave rise to the Einstein and Gauss-Bonnet 3 point functions were unique, the Lagrangians that give rise to the $R^{3} 3$ point function have a one parameter ambiguity, parameterized by the coefficient of the second Lovelock terms - which is an $R^{3}$ term whose contribution to the 3 graviton S-matrix vanishes. It is thus clear that the 4 point function that follows from the exchange of such a vertex has a contribution from the 2 nd Lovelock term with an undetermined coefficient.
} 


\begin{tabular}{|l|l|l|}
\hline Exchange & Regge behavior (large $s$, fixed $t$ ) & Regge behavior after subtraction \\
\hline $\mathcal{A}_{R-R}$ & $s^{2} / t$ & - \\
\hline $\mathcal{A}_{R-R^{2}}$ & $s^{2}$ & - \\
\hline $\mathcal{A}_{R-R^{3}}$ & $s^{2} t$ & - \\
\hline $\mathcal{A}_{R^{2}-R^{2}}$ & $s^{3}$ & - \\
\hline $\mathcal{A}_{R^{2}-R^{3}}$ & $s^{4}$ & $s^{3} t$ \\
\hline $\mathcal{A}_{R^{3}-R^{3}}$ & $s^{5}$ & $s^{4} t$ \\
\hline
\end{tabular}

Table 8. Regge behavior of exchange diagrams.

$$
\begin{aligned}
& +\frac{3}{2} \nabla^{\mu} \nabla^{\nu} R_{\text {pqrs }} R_{p q t u} \nabla^{\mu} R_{r t v w} \nabla^{\nu} R_{\text {suvw }} \\
& -10 \nabla_{\mu} \nabla_{\nu} R_{\text {pqrs }} \nabla^{\mu} \nabla_{\nu} R_{p t r u} R_{t v u w} R_{q v s w} \\
& -11 \nabla_{\mu} \nabla_{\nu} R_{\text {pqrs }} \nabla_{\nu} R_{p t r u} R_{t v u w} \nabla^{\mu} R_{q v s w} \\
& +10 \nabla_{\mu} \nabla_{\nu} R_{\text {pqrs }} \nabla_{\mu} R_{p t u w} \nabla_{\nu} R_{t v w s} R_{q v r u} \\
& +\frac{1}{8} \nabla^{\mu} \nabla^{\nu} R_{a b c d} R_{a b c d} R_{p q r s} \nabla_{\mu} \nabla_{\nu} R_{p q r s} \\
& +\frac{5}{16} \nabla^{\mu} \nabla^{\nu} R_{a b c d} R_{a b c d} \nabla_{\nu} R_{p q r s} \nabla_{\mu} R_{p q r s} \\
& -\frac{1}{4} \nabla_{\mu} \nabla_{\nu} R_{p q r s} \nabla^{\mu} \nabla^{\nu} R_{p q t u} R_{t u v w} R_{r s v w} \\
& -\frac{3}{8} \nabla_{\mu} \nabla_{\nu} R_{p q r s} \nabla^{\nu} R_{p q t u} R_{t u v w} \nabla^{\mu} R_{r s v w} \\
& -32 \nabla_{\mu} R_{\alpha \beta a b} \nabla^{\mu} \nabla_{a} R_{\beta \gamma c d} \nabla_{b} R_{\gamma \delta d e} R_{\delta \alpha e c} \\
& \left.-\frac{8}{3} R_{a b c d} \nabla_{a} \nabla_{c} R_{\alpha \beta \gamma \delta} \nabla_{b} \nabla_{d} R_{\beta \mu \delta \nu} R_{\mu \alpha \nu \gamma}\right)
\end{aligned}
$$

While it is not manifest from the expressions above, we have checked that all scattering amplitudes involving the Gauss-Bonnet 3 point function vanishes for $D=4$, as expected (recall the Gauss-Bonnet Lagrangian is topological in 4 dimensions; in particular its contribution to 3 graviton scattering vanishes).

Regge growth. The Regge behavior of the amplitudes constructed in this section is easily determined. ${ }^{104}$ In every case the ' $t$ channel contributions' (i.e. the terms in the Smatrix that are non polynomial in $t$ when expressed as functions of particle momenta and $\epsilon_{i}$ ) grow no faster than $s^{2}$, consistent with the fact that we are studying the exchange of a spin 2 particle. All other contributions to the $S$ matrix are analytic in $t$. It follows from dimensional analysis that these remaining contributions can grow no faster than $s$ (in the

\footnotetext{
${ }^{104}$ As usual one obtains the Regge behavior by explicitly decomposing the polarizations into transverse and parallel components using (2.11) and (2.13) and evaluating the resulting S-matrix at large $s$, keeping $\mathrm{t}$ fixed.
} 
case of $\mathcal{A}_{R-R}$ ), or $s^{2}$ (in the case of $\mathcal{A}_{R-R^{2}}$ ). Dimensional analysis would have allowed $\mathcal{A}_{R-R^{3}} s^{3}$ growth but we find that the amplitude actually grows more slowly like $s^{2} t$.

In the case of $\mathcal{A}_{R^{2}-R^{2}}$ the sum of $s$ and $u$ channel exchanges gives rise to an S-matrix that is 6th order in derivatives and grows like $s^{3}-$ and so faster than $s^{2}-$ in the Regge limit. It is easy to see that this faster than $s^{2}$ growth cannot be canceled by a local counterterm. This can be seen in two equivalent ways. First, in our exhaustive classification of local counter-terms earlier in this paper there is only one S-matrix that is of sixth order in derivatives, and this S-matrix grows like $s^{2} t$ rather than like $s^{3}$ in the Regge limit. Equivalently, we have explicitly constructed the Lagrangian that gives rise to the $\mathcal{A}_{R^{2}-R^{2}}$ S-matrix (see (7.8)) and it simply is not local, even in the Regge limit.

In the case of $\mathcal{A}_{R^{3}-R^{3}}$ the S-matrix is 10 th order in derivatives and grows like $s^{5}-$ and so considerably faster than $s^{2}$ - in the Regge limit. This growth can be slightly ameliorated by counter-term subtractions. The explicit S-matrix for this term is listed in (7.7). Notice that in (7.7) the functions $\mathcal{F}$ are all polynomials. It follows that all the contributions from these functions can be canceled by local counter-terms. The only piece in $\mathcal{A}_{R^{3}-R^{3}}$ that cannot be cancelled by a local counter-term is the part of the S-matrix parameterized by $\mathcal{F}^{G_{3,1}}(t, u)=\frac{-9\left(t^{2}+u^{2}+D u t\right)}{64(D-2) s}$. After a further local counter-term subtraction we are left with $\mathcal{F}^{G_{3,1}}(t, u) \propto \frac{t u}{t+u}$. In both the $u$ and the $s$ channels the subtracted $\mathcal{F}^{G_{3,1}}(t, u)$ is now proportional to $t$ in the Regge limit, resulting in a (maximally subtracted) scattering amplitude that scales like $s^{4} t$ in the Regge limit.

Finally, in case of $\mathcal{A}_{R^{2}-R^{3}}$ the explicit S-matrix (see (7.7)) is of 8 derivative order and grows like $s^{4}$. Once again counter-term subtractions can be used to reduce this growth down to $s^{3} t$. In particular, the contribution to this S-matrix from $\mathcal{F}^{G, 7}(t, u)=\frac{3}{2 s}-$ a term which clearly cannot be cancelled by a local counter-term - grows like $s^{3} t$. It follows that local counter-terms cannot be used to further reduce the Regge growth of this S-matrix.

Our final results are summarized in table 8. Plugging the results of table 8 into (7.6) we conclude that the only graviton exchange contributions (7.6) that grow no faster than $s^{2}$ in the Regge limit are those with $\alpha_{R^{2}}=\alpha_{R^{3}}=0 .{ }^{105,106}$

The uniqueness of Einstein gravity three point function has also been argued in [8] on account of violation of the "superconvergence sum rule" for non-zero $\alpha_{R^{2}}$ or non-zero $\alpha_{R^{3}}$.

\subsection{4 graviton scattering from exchanges of massive particles}

In this section we compute the pole contributions to four graviton scattering from

- The exchange of a massive scalar.

- The exchange of a massive spin 2 particle.

- The exchange of a massive spin 1 particle, assuming that the graviton - graviton spin $l$ three point S-matrix takes one of three kinematically allowed forms.

\footnotetext{
${ }^{105}$ While $\mathcal{A}_{R-R}, \mathcal{A}_{R-R^{2}}$ and $\mathcal{A}_{R-R^{3}}$ grow like $s^{2}$ the remaining 3 amplitudes grow faster than $s^{2}$. The fact that the coefficient of $\mathcal{A}_{R^{2}-R^{2}}$ must vanish forces $\alpha_{R^{2}}$ to vanish. The fact that the coefficient of $\mathcal{A}_{R^{3} R^{3}}$ must also vanish forces $\alpha_{R^{3}}$ to vanish.

${ }^{106}$ Also note that the six derivative contact term ambiguity that one encounters in $\mathcal{A}_{R-R^{3}}$, scales as stu and hence is Regge allowed.
} 
The chief qualitative observation we will make is that (in contrast with the scalar and photon case) all of the exchange contributions to four graviton scattering that we compute grow faster than $s^{2}$ in the Regge limit even after accounting for possible local counter-term subtractions.

\subsubsection{Massive scalar exchange}

A three point scattering amplitude between two gravitons and a scalar is a polynomial in $\epsilon_{1}, \epsilon_{2}, k_{1}$ and $k_{2}$ that is of second order in $\epsilon_{1}$ and $\epsilon_{2}$ and is also invariant under the Bose flip $1 \leftrightarrow 2$ of the external gravitons. We consider three point functions for which the external gravitons are on-shell but the intermediate particle (that is being exchanged) is allowed to be off-shell (or, equivalently, is of indeterminate mass). In other words the expressions we construct will not be functions of $p_{1}^{2}$ and $p_{2}^{2}$ (because these vanish) but will be allowed to depend on $p_{1} \cdot p_{2}$. The most general expression that meets these conditions is given by

$$
A\left(\epsilon_{1} \cdot p_{2}\right)^{2}\left(\epsilon_{2} \cdot p_{1}\right)^{2}+B\left(\epsilon_{1} \cdot \epsilon_{2}\right)^{2}+C\left(\epsilon_{1} \cdot \epsilon_{2}\right)\left(\epsilon_{1} \cdot p_{2}\right)\left(\epsilon_{2} \cdot p_{1}\right)
$$

Where $A, B$ and $C$ are arbitrary functions of $p_{1} \cdot p_{2}$. The requirement that the S-matrix also enjoys invariance under $\epsilon_{i} \rightarrow \epsilon_{i}+c_{i} k_{i}$ fixes the three point function to take the form

$$
A\left(\epsilon_{1} \cdot p_{2} \epsilon_{2} \cdot p_{1}-p_{1} \cdot p_{2} \epsilon_{1} \cdot \epsilon_{2}\right)^{2}=A R_{1}^{\mu \nu \alpha \beta} R_{2}^{\mu \nu \alpha \beta}
$$

where, once again, $A$ is an arbitrary function of $p_{1} . p_{2}$. Eq. (7.18) is off-shell gauge invariant for every choice of the function $A$. Different choices of $A$ yield four graviton exchange amplitudes that differ only in polynomial pieces. For simplicity we choose $A$ to be a constant. The Lagrangian that generates the scattering amplitude (7.18) is given by

$$
R_{a b c d} R^{a b c d} \phi
$$

where $\phi$ is the massive scalar field.

The S-matrix that follows by stitching together two copies of (7.18) through a scalar propagator is proportional to $S^{G_{3,1}}$ (see (K.1)) with

$$
\mathcal{F}^{G_{3,1}}(t, u)=\frac{1}{s-m^{2}} .
$$

The S-matrix described above is formally generated by the non local Lagrangian

$$
\mathcal{A}=\frac{1}{s-m^{2}}\left(R_{a b c d} R_{a b c d} R_{p q r s} R_{p q r s}\right) .
$$

Regge growth. In the Regge limit i.e. large $s$ fixed $t$, it is easily verified that the amplitude (7.20) grows like $s^{3}$. To be precise, we analyze the Regge behavior from each channel:

$$
\begin{aligned}
& \mathcal{S}_{s} \rightarrow s^{3}\left(\left(-1+\epsilon_{1}^{\perp} \cdot \epsilon_{2}^{\perp}\right)^{2}\left(-1+\epsilon_{3}^{\perp} \cdot \epsilon_{4}^{\perp}\right)^{2}\right) \\
& \mathcal{S}_{t} \rightarrow t^{4}\left(\left(-1+\epsilon_{1}^{\perp} \cdot \epsilon_{3}^{\perp}\right)^{2}\left(-1+\epsilon_{2}^{\perp} \cdot \epsilon_{4}^{\perp}\right)^{2}\right) \\
& \mathcal{S}_{u} \rightarrow-s^{3}\left(\left(-1+\epsilon_{1}^{\perp} \cdot \epsilon_{4}^{\perp}\right)^{2}\left(-1+\epsilon_{2}^{\perp} \cdot \epsilon_{3}^{\perp}\right)^{2}\right) .
\end{aligned}
$$


Notice that the $t$ channel contribution grows like $s^{0}$ (in agreement with general expectations for the $t$ channel growth in the exchange of a spin zero particle)., The leading Regge growth is from the $\mathrm{s}$ and $\mathrm{u}$ channel, but they arise with opposite signs, and naively we may think that they might cancel. However, their growth comes with different polarization vectors, and hence cannot cancel for generic choice of the polarization vectors. Hence, the Regge growth for the amplitude is $s^{3}$, which arises from $\mathrm{s}$ and $\mathrm{u}$ channels. In the Regge limit $\mathcal{F}^{G_{3,1}}(t, u)=\frac{1}{s}$. The only available 6 derivative local counter-term is $G_{\mathbf{S}, 1}$, and the Smatrix it gives rise to does not cancel that coming from (7.20). It follows that the $s^{3}$ Regge growth from scalar exchange is irreducible (i.e. cannot be further reduced by local counter-term subtraction).

\subsubsection{Massive spin 2 exchange}

We now consider the contribution of an exchange of a massive spin two particle to four graviton scattering. The massive spin 2 field $S_{\mu \nu}$ is symmetric and traceless. We take its polarization tensor to be $\epsilon_{3}^{\mu} \epsilon_{3}^{\nu}$ and its momentum to be $p_{3}$. Note that $\epsilon_{3} . \epsilon_{3}=0$ and $p_{3} . \epsilon_{3}=0$.

The independent data for the scattering amplitude is given by five vectors; $p_{1}, p_{2}, \epsilon_{1}$, $\epsilon_{2}$ and $\epsilon_{3}$. Recall that the final S-matrix is a scalar. Any scalar built out of a set of vectors is a polynomial in the seven independent dot products of these vectors We start by writing the Lorentz invariant building blocks involving the three particles:

$$
\begin{array}{lll}
A_{1}=\epsilon_{1} \cdot p_{2} & A_{2}=\epsilon_{2} \cdot p_{1} \quad A_{3}=\epsilon_{3} \cdot\left(p_{1}-p_{2}\right) \\
b_{12}=\epsilon_{1} \cdot \epsilon_{2} & b_{23}=\epsilon_{2} \cdot \epsilon_{3} & b_{13}=\epsilon_{1} \cdot \epsilon_{3}
\end{array}
$$

Note that $A_{3}$ is antisymmetric under $1 \leftrightarrow 2$. The most general polynomial built out of (7.25) that is of homogeneity two in each of $\epsilon_{1}, \epsilon_{2}$ and $\epsilon_{3}$ and also has the $1 \leftrightarrow 2$ Bose exchange symmetry is a linear combination of the structures

$$
\begin{aligned}
& A=b_{12} b_{23} b_{13} \\
& B=b_{13} b_{23} A_{1} A_{2} \\
& C=b_{13}^{2} A_{2}^{2}+b_{23}^{2} A_{1}^{2} \\
& D=b_{23} b_{12} A_{1} A_{3}-b_{13} b_{12} A_{2} A_{3} \\
& E=A_{3}^{2} b_{12}^{2} \\
& F=A_{3} b_{23} A_{2} A_{1}^{2}-A_{3} b_{13} A_{1} A_{2}^{2} \\
& G=A_{3}^{2} A_{1} A_{2} b_{12} \\
& H=A_{3}^{2} A_{1}^{2} A_{2}^{2}
\end{aligned}
$$

where the coefficients of the linear combination are arbitrary functions of $p_{1} \cdot p_{2}$. It is not difficult to verify that only two linear combinations of (7.26) are invariant under $\epsilon_{1} \rightarrow$ $\epsilon_{2}+c_{1} p_{1}$ and $\epsilon_{2} \rightarrow c_{2} p_{2}$. One of the two gauge invariant S-matrices occurs at four derivative order and is given by $\left(p_{1} \cdot p_{2}\right)^{2} A-\left(p_{1} \cdot p_{2}\right) B-\frac{1}{2}\left(p_{1} \cdot p_{2}\right) D-\frac{1}{4}\left(p_{1} \cdot p_{2}\right) E+\frac{1}{2} F+\frac{1}{4} G$, or more 
explicitly by

$$
\left(p_{1} . \epsilon_{2} p_{2} . \epsilon_{1}-p_{1} . p_{2} \epsilon_{1} . \epsilon_{2}\right)\left(\epsilon_{1} . \epsilon_{3}\left(p_{1} . \epsilon_{2} p_{2} . \epsilon_{3}-p_{1} \cdot p_{2} \epsilon_{2} . \epsilon_{3}\right)+p_{1} . \epsilon_{3}\left(p_{2} . \epsilon_{1} \epsilon_{2} . \epsilon_{3}-\epsilon_{1} \cdot \epsilon_{2} p_{2} . \epsilon_{3}\right)\right)
$$

The 3 particle S-matrix (7.27) may be rewritten in terms of field strengths as

$$
F_{1}^{a b} F_{2}^{a b} F_{1}^{c \mu} F_{2}^{c \nu} S^{\mu \nu}
$$

The Lagrangian that yields this 3 point function is:

$$
R_{a b c \mu} R_{\nu}^{a b c} S^{\mu \nu}
$$

It is not difficult to check that the 3 point function (7.27) may, in fact, be rewritten as

$$
\left(\epsilon_{1} \wedge \epsilon_{2} \wedge \epsilon_{3} \wedge p_{1} \wedge p_{2}\right)^{2}
$$

and so is identical in structure to the 3 graviton scattering amplitude from the GaussBonnet term (4.23).

There is another gauge invariant combination of the building blocks, at the level of six derivatives, which is $\frac{1}{2}\left(p_{1} \cdot p_{2}\right)^{2} E-\left(p_{1} \cdot p_{2}\right) G+\frac{1}{2} H$, or more explicitly by

$$
A_{6}=\left(p_{1} . \epsilon_{3} p_{2} . \epsilon_{3}\right)\left(p_{1} . \epsilon_{2} p_{2} . \epsilon_{1}-p_{1} \cdot p_{2} \epsilon_{1} . \epsilon_{2}\right)^{2}
$$

In terms of the Field strength, the above structure becomes:

$$
-p_{1}^{\mu} F_{1}^{a b} F_{2}^{a b} p_{2}^{\nu} F_{1}^{c d} F_{2}^{c d} S^{\mu \nu}
$$

The Lagrangian structure which yields this 3 point function is:

$$
B=\nabla_{\mu} R^{a b c d} \nabla_{\nu} R_{a b c d} S^{\mu \nu}
$$

Formally defining $F_{3}^{\mu \nu}$ it is not difficult to verify that $A_{6}$ may be rewritten as

$$
A_{6} \propto\left(\operatorname{Tr} F_{1} F_{2} F_{3}\right)^{2}
$$

and so is the analogue of the 3 graviton scattering amplitude (4.24).

Note that the three point functions from (7.29) and (7.33) are both gauge invariant even off-shell (i.e. without using $p_{1}^{2}=p_{2}^{2}=0$ ). ${ }^{107}$

In summary, there are exactly two graviton - graviton - massive spin-2 3-point functions. The first is the analogue of the Gauss-Bonnet 3 point coupling for three gravitons. The second is the analogue of the Riemann cube 3 point coupling for 3 gravitons. In the case that particle 3 is massive, there is no analogue of the Einstein 3 point coupling for 3 gravitons. At least at the algebraic level the lack of a structure analogous to the Einstein 3

\footnotetext{
${ }^{107}$ In fact the amplitudes are even formally off-shell gauge invariant under fictitious gauge transformations for the particle 3. This observation is not really physical as, on-shell, the transformation $\epsilon_{3} \rightarrow \epsilon_{3}+p_{3}$ does not preserve the condition $\epsilon_{3} . p_{3}=0$ as particle 3 is massive.
} 
graviton coupling is a consequence of the fact that (4.22) is gauge invariant only on-shell; the manipulations that ensure this make crucial use of the fact that $p_{3}^{2}=0$ and have no extension to $p_{3}^{2} \neq 0 .{ }^{108}$

In summary, the most general 3 point function is given by

$$
\alpha_{4} A_{4}+\alpha_{6} A_{6}
$$

The most general massive spin two exchange contribution to four graviton scattering is thus given by stitching these two three point functions with a massive spin-2 propagator

$$
P_{\mu_{1} \mu_{2}, \nu_{1} \nu_{2}}(p)=\frac{1}{p^{2}+m^{2}}\left(\frac{1}{2}\left(\theta_{\mu_{1} \nu_{1}} \theta_{\mu_{2} \nu_{2}}+\theta_{\mu_{1} \nu_{2}} \theta_{\mu_{2} \nu_{1}}\right)-\frac{1}{D-1} \theta_{\mu_{1} \mu_{2}} \theta_{\nu_{1} \nu_{2}}\right)
$$

and yields a 4 graviton scattering amplitude of the form

$$
\mathcal{A}=\alpha_{4}^{2} \mathcal{A}_{4,4}+\alpha_{4} \alpha_{6} \mathcal{A}_{4,6}+\alpha_{6}^{2} \mathcal{A}_{6,6}
$$

The explicit expressions for $\mathcal{A}_{4,4}, \mathcal{A}_{4,6}$ and $\mathcal{A}_{6,6}$ are easy to obtain and are given by

$$
\begin{aligned}
& \mathcal{A}_{A-A}=\left\{\begin{array}{l}
S^{G_{\mathbf{3}, 6}}+S^{G_{\mathbf{3}, 1}}, \\
\mathcal{F}^{G_{\mathbf{3}, 6}}(s, t)=\frac{1}{4\left(s-m^{2}\right)}, \mathcal{F}^{G_{\mathbf{3}, 1}}(t, u)=\frac{8+D}{16(1-D)\left(s-m^{2}\right)}
\end{array}\right. \\
& \mathcal{A}_{A-B}=\left\{\begin{array}{l}
S^{G_{3,5}}+S^{G_{3}, 1}+S^{G_{3}, 2}+S^{G_{3}, 7}+S^{G_{\mathbf{3}} \mathbf{A}}, \\
\mathcal{F}^{G_{3,5}}(t, u)=\frac{s}{2\left(s-m^{2}\right)}, \mathcal{F}^{G_{3,1}}(t, u)=\frac{3 s}{8(-1+D)\left(s-m^{2}\right)}, \\
\mathcal{F}^{G_{3,2}}(t, u)=\frac{s}{8\left(s-m^{2}\right)}, \mathcal{F}^{G_{3,7}}(t, u)=\frac{4}{s-m^{2}}, \mathcal{F}^{G_{\mathbf{3}^{\mathbf{A}}}}(t, u)=\frac{2(t-u)}{4\left(s-m^{2}\right)}
\end{array}\right. \\
& \mathcal{A}_{B-B}=\left\{\begin{array}{l}
S^{G_{3,1}}, \\
\mathcal{F}^{G_{3,1}}(t, u)=\frac{(-2+D)\left(u^{2}+t^{2}\right)-2 D u t}{16(-1+D)\left(s-m^{2}\right)} .
\end{array}\right.
\end{aligned}
$$

The non-local Lagrangians which generate these amplitudes are quite non-trivial functions

\footnotetext{
${ }^{108}$ Under the gauge transformation $\epsilon_{1} \rightarrow \epsilon_{1}+\lambda p_{1}$, this is proportional to

$$
\left(p_{1} \cdot p_{2} \epsilon_{2} \cdot \epsilon_{3}\right)
$$

which vanishes only on-shell. Hence there are no possible gauge-invariant massive deformations of the three point function coming from Einstein gravity.
} 
of the mass $m$.

$$
\begin{aligned}
\left(s-m^{2}\right)\left(t-m^{2}\right)\left(u-m^{2}\right) \mathcal{A}_{A-A}= & \left(\frac{1}{2} \nabla_{\mu} \nabla_{\nu} R_{p q r s} R_{p q r t} \nabla^{\mu} R_{u v w t} \nabla^{\nu} R_{u v w s}\right. \\
& -\frac{m^{2}}{2} \nabla_{\mu} R_{p q r s} R_{p q r t} \nabla^{\mu} R_{u v w t} R_{u v w s} \\
& -\frac{m^{2}}{2} \nabla_{\nu} R_{p q r s} R_{p q r t} R_{u v w t} \nabla^{\nu} R_{u v w s} \\
& +\frac{m^{4}}{2} R_{p q r s} R_{p q r t} R_{u v w t} R_{u v w s} \\
& +\frac{(D+8)}{32(1-D)} \nabla_{\mu} \nabla_{\nu} R_{a b c d} R_{a b c d} \nabla^{\mu} R_{p q r s} \nabla^{\nu} R_{p q r s} \\
& -\frac{m^{2}(D+8)}{32(1-D)} \nabla_{\mu} R_{a b c d} R_{a b c d} \nabla^{\mu} R_{p q r s} R_{p q r s} \\
& -\frac{m^{2}(D+8)}{32(1-D)} \nabla_{\nu} R_{a b c d} R_{a b c d} R_{p q r s} \nabla^{\nu} R_{p q r s} \\
& \left.+\frac{m^{4}(D+8)}{32(1-D)} R_{a b c d} R_{a b c d} R_{p q r s} R_{p q r s}\right)
\end{aligned}
$$

The reader can check the Lagrangian (7.39) indeed generates the S-matrix of $\mathcal{A}_{A-A}$ in the first line of (7.38). The product factor $\left(t-m^{2}\right)\left(u-m^{2}\right)$ cancels with the contribution of the $m^{2}$ and $m^{4}$ terms in (7.39) to give the required momenta functions in (7.38). To avoid clutter, we suppress the $O\left(m^{2}, m^{4}\right)$ terms in writing down the non-local Lagrangians for $\mathcal{A}_{A-B}$ and $\mathcal{A}_{B-B}$

$$
\begin{aligned}
\left(s-m^{2}\right)\left(t-m^{2}\right)\left(u-m^{2}\right) \mathcal{A}_{A-B}= & \left(\nabla_{\gamma} \nabla_{\delta} \nabla^{\mu} R_{p q r s} \nabla_{\mu} R_{p q r t} \nabla^{\gamma} R_{u v w t} \nabla^{\delta} R_{u v w s}\right. \\
& +2 \nabla_{\gamma} \nabla_{\delta} \nabla^{\mu} R_{p q r s} R_{p q r t} \nabla_{\mu} \nabla^{\gamma} R_{u v w t} \nabla^{\delta} R_{u v w s} \\
& -\nabla_{\gamma} \nabla_{\delta} \nabla^{\mu} R_{p q r s} R_{p q t u} \nabla_{\mu} \nabla^{\gamma} R_{r t v w} \nabla^{\delta} R_{\text {suvw }} \\
& +\frac{3}{4(D-1)} \nabla_{\gamma} \nabla_{\delta} \nabla^{\mu} R_{p q r s} R_{p q r s} \nabla_{\mu} \nabla^{\gamma} R_{a b c d} \nabla^{\delta} R_{a b c d} \\
& -\frac{1}{4} \nabla_{\gamma} \nabla_{\delta} \nabla_{\mu} R_{p q r s} \nabla^{\mu} R_{p q t u} \nabla^{\gamma} R_{t u v w} \nabla^{\delta} R_{r s v w} \\
& \left.-2 \nabla_{\gamma} \nabla_{\delta} R_{\mu \nu a b} \nabla_{a} R_{\nu \mu m n} \nabla_{b} \nabla^{\gamma} R_{\alpha \beta n p} \nabla^{\delta} R_{\beta a p m}+\mathcal{O}\left(m^{2}\right)\right) \\
\left(s-m^{2}\right)\left(t-m^{2}\right)\left(u-m^{2}\right) \mathcal{A}_{B-B}= & \left(\frac{D-2}{4(D-1)} \nabla^{\mu} \nabla^{\nu} \nabla^{\gamma} \nabla^{\delta} R_{a b c d} R_{a b c d} \nabla_{\gamma} R_{m n o p} \nabla_{\mu} \nabla_{\nu} \nabla_{\delta} R_{m n o p}\right. \\
& +\frac{D}{4(1-D)} \nabla^{\mu} \nabla^{\nu} \nabla^{\gamma} \nabla^{\delta} R_{a b c d} R_{a b c d} \nabla_{\mu} \nabla_{\nu} R_{m n o p} \nabla_{\gamma} \nabla_{\delta} R_{m n o p} \\
& \left.+\mathcal{O}\left(m^{2}\right)\right)
\end{aligned}
$$

Regge growth. The analysis (and final results) of Regge behaviors of the amplitudes $\mathcal{A}_{A-A}, \mathcal{A}_{A-B}$ and $\mathcal{A}_{B-B}$ is essentially identical in structure to the analysis of the Regge 


\begin{tabular}{|l|l|l|}
\hline Exchange & Regge behavior (large $s$, fixed $t$ ) & Regge behavior after subtraction \\
\hline $\mathcal{A}_{R R \phi}$ & $s^{3}$ & - \\
\hline $\mathcal{A}_{A-A}$ & $s^{3}$ & - \\
\hline $\mathcal{A}_{A-B}$ & $s^{4}$ & $s^{3} t$ \\
\hline $\mathcal{A}_{B-B}$ & $s^{5}$ & $s^{4} t$ \\
\hline
\end{tabular}

Table 9. Regge behavior of exchange diagrams for massive particles.

growth in $\mathcal{A}_{R^{2}-R^{2}}, \mathcal{A}_{R^{2}-R^{3}}$ and $\mathcal{A}_{R^{3}-R^{3}}$. The Regge behavior of the different amplitudes is listed in table 9 .

\subsection{Exchange contribution to gravitational scattering and Regge growth}

Above we have computed the contributions to graviton scattering from the exchange of massive scalars and massive spin two particles (we have also computed one series of exchange contributions from the exchange of massive spin $2 l$ particles). In each case we have seen that the exchange contributions grow faster than $s^{2}$ in the Regge limit, and also that this growth cannot be sufficiently tamed (i.e. brought down to growth like $s^{2}$ or slower) by the subtraction of local counter-term contributions. In this subsection we argue (under a plausible but not completely justified assumption) that this feature is general: it applies to the contribution to four graviton scattering from the exchange of any particle, atleast when $D \leq 6$.

\subsubsection{Regge growth of general exchange contributions}

The contribution to gravitational scattering of the exchange of a massive particle of any spin takes the form

$$
\mathcal{S}=\frac{\left|\alpha_{1}\right\rangle}{s-m^{2}}+\frac{\left|\alpha_{2}\right\rangle}{t-m^{2}}+\frac{\left|\alpha_{3}\right\rangle}{u-m^{2}} .
$$

Here $\left|\alpha_{i}\right\rangle$ are elements of the local Module (this follows from the fact that 3 point functions are local and gauge invariant).

Let us suppose that $\left|\alpha_{i}\right\rangle$ are of $2 n^{\text {th }}$ order in derivatives. It follows that

$$
\left(s-m^{2}\right)\left(t-m^{2}\right)\left(u-m^{2}\right) \mathcal{S}
$$

is local, and of degree $2 n+4$ in derivatives. We note for later use that the part of (7.42) that is of order $2 n+4$ in derivative is given by

$$
\text { stuS }
$$

It follows that the growth of (7.42) is no slower that $s^{\alpha(n+2)+\frac{a}{3}}$ where $\alpha(n)$ is listed in (2.66). It then follows that the growth of $\mathcal{S}$ in the Regge limit is at least as fast as $s^{\alpha(n-1)+\frac{a}{3}}$. In the special case that $a=0$ the exchange contributions always grow faster than $s^{2}$ whenever $n-1>3$, i.e. for $n>4$. 
Let us now focus on the borderline 'dangerous' case $n=4$. In this case we obtain an exchange S-matrix $\mathcal{S}$ that grows like $s^{2}$ in the Regge limit only when the quantity in (7.43) is of the form

$$
3(s t u)^{2}\left|g_{\mathbf{S}}\right\rangle
$$

where $\left|g_{\mathbf{S}}\right\rangle$ is a symmetric generator of the bare module (see subsection 2.10). ${ }^{109}$ When this is the case the S-matrix is given by

$$
\mathcal{S}=3(s t u)\left|g_{\mathbf{S}}\right\rangle
$$

Comparing (7.44) and (7.41) we conclude that an exchange S-matrix can have the 'dangerous' $s^{2}$ growth only if and only if the module elements $\left|\alpha_{i}\right\rangle$ take the form

$$
\left|\alpha_{1}\right\rangle=s(s t u)\left|g_{\mathbf{S}}\right\rangle, \quad\left|\alpha_{2}\right\rangle=t(s t u)\left|g_{\mathbf{S}}\right\rangle, \quad\left|\alpha_{3}\right\rangle=u(s t u)\left|g_{\mathbf{S}}\right\rangle .
$$

\subsubsection{Structure of $\left|\alpha_{i}\right\rangle$ for the case of gravitational scattering}

Let us now specialize to the special case of exchange contributions to four graviton scattering by a particle of general spin, $\chi_{m n \ldots}$. We expect - and assume - that the three point function between $\chi_{m n \ldots}$ and two gravitons to take the schematic form

$$
\chi_{m n \ldots} R_{a b c d} R_{e f g h}
$$

where all indices are appropriately contracted and the three point functions may also involve extra derivatives. ${ }^{110}$ The contribution of the exchange of $\chi_{m n \ldots}$ to four graviton scattering thus leads to an S-matrix of the form (7.41) with $\left|\alpha_{i}\right\rangle$ given by descendants of four Riemann structures, i.e. by elements of the module described in subsubsection 6.1.1.

We have argued in the previous subsubsection that such a contribution can grow like $s^{2}$ or slower only if $\left|\alpha_{i}\right\rangle$ are 8 derivative objects (i.e. are linear combinations of the four Riemann generators described in subsubsection 6.1.1) and additionally if (7.45) holds. However the only multiplet of four $\mathrm{R}$ structures that is of the form (7.45) are the descendants of $\left|G_{\mathbf{S}, 1}\right\rangle$ (see subsubsection 6.1.1, in particular see (6.3)). ${ }^{111}$ We conclude that the only possible exchange contribution that grows no faster than $s^{2}$ in the Regge limit is one proportional to the S-matrix from $G_{\mathbf{S}, 1}$. It follows, in particular, that all exchange contributions to graviton scattering in $D \leq 6$ grow faster than $s^{2}$ in the Regge limit.

Note that while we have not been able to rule out the possibility of an exchange contribution proportional to $G_{\mathbf{S}, 1}$ in $D \geq 7$, it is entirely possible that such a term is never actually generated. ${ }^{112}$ We leave the careful investigation of this point to the future.

\footnotetext{
${ }^{109}$ The factor of 3 is inserted for later algebraic convenience.

${ }^{110}$ Note that a coupling of the schematic form $\chi_{m n n . .} R_{a b c d}$ induces mixing between $\chi_{m n \ldots}$ and the graviton at quadratic order. Such couplings are eliminated by field redefinitions. The lowest order couplings that survive after field redefinitions render the Lagrangian diagonal at quadratic level are those of the form (7.46).

${ }^{111}$ The fact that no other multiplet of four Riemann structures are of the form (7.45) follows from the fact that the module $M_{8}$ described in subsubsection 6.1.1 is freely generated. Had another relation like (6.3) existed, there would have been null states in $M_{8}$ - of exactly the same form as the null states of $M_{8}^{\prime}$.

${ }^{112}$ Such a term can only be generated $\left|\alpha_{1}\right\rangle=s\left|G_{\mathbf{S}, 1}\right\rangle$ is a sum of 'perfect squares'; it is entirely possible that this is not the case. We hope to address this issue in the future.
} 


\subsubsection{Counter-term cancellation}

Say we have an exchange contribution that grows faster than $s^{2}$ in the Regge limit. In this section we investigate whether its growth can be cancelled by a local counter-term.

Let us once again focus on S-matrices of the form (7.41), and focus on the part of $\left|\alpha_{i}\right\rangle$ that is of 8 th order in derivatives. We have just argued that all such terms grow faster than $s^{2}$ in $D \leq 6$. The denominator in (7.41) turns the 8 derivative numerator into a six derivative $\mathrm{S}$ matrix. It is immediately clear in $D \leq 6$ that this six derivative term cannot be cancelled by local counter-terms, simply because we have carefully enumerated all available counter-terms earlier in this paper, and all these counter-terms are of 8 or higher order in derivatives when $D \leq 6$.

It follows that it is impossible to use local counter-terms to cancel the offending large $s$ behavior of exchange diagrams unless the 8 derivative part of $\left|\alpha_{i}\right\rangle$ vanish. It seems extremely unlikely that this can happen unless $\left|\alpha_{i}\right\rangle$ itself vanishes. ${ }^{113}$

\subsubsection{Cancellation between exchange diagrams}

The reader may wonder whether the offending Regge behavior in exchange contributions to gravitational scattering can cancel between themselves. Could, for instance, the contribution from the exchange of a particle at mass $m_{1}$ in some representation cancel offending part from the exchange of a particle of mass $m_{2}$ in the same representation? We believe this cannot happen for the reasons we now describe.

When the particle exchanged lies in a representation with four or more symmetrized Lorentz indices, it is kinematically obvious that cancellation cannot sufficiently improve Regge behavior. This is because the exchange of such particles lead to violation of $s^{2}$ growth even in the $t$ channel. The violating contribution in this channel scales like

$$
\frac{s^{l}}{t-m^{2}}
$$

As the functional form of this amplitude is a function of $t$ with complicated $m^{2}$ dependence, it is obvious that the Regge growths of particles of different mass cannot cancel each other.

When the particle exchanged lies in a representation with three or fewer symmetrized indices, the faster than $s^{2}$ Regge growth appears in the $s$ and $u$ channels. The dependence of these violations on $m^{2}$ are relatively simple. Even though this is the case, two different exchange contributions cannot cancel against each other, simply because each exchange contribution is a perfect square; contributions that are proportional to each other are all of the same sign, and so can only add and never cancel. The positivity demanded above follows from the requirement that all exchange particles have the right sign kinetic term, and that all three point couplings are real - these are both constraints that any sensible classical theory should clearly have.

\footnotetext{
${ }^{113}$ Exchange contributions are not homogeneous in derivatives. An $\left|\alpha_{i}\right\rangle$ that, for instance, starts out at 10th order in derivatives also has a piece at 8 th order in derivatives obtained by Taylor expanding the answer in $m^{2}$.
} 


\section{Discussion}

Even though this paper and related appendices runs over more than a hundred and fifty pages a great deal remains to be done.

The principal technical accomplishment of this paper is the detailed classification of all polynomial four photon and four graviton S-matrices. ${ }^{114}$ The exhaustive classification presented in this paper has been arrived at with the aid of some guided guesswork. While our final results have passed several non-trivial consistency checks and so are very likely correct, it would be useful to re-derive our classification in a mathematically rigorous manner. The methods of [10] and [11] may prove useful in this regard.

It would be useful - and should not be difficult - to complete the detailed classification of exchange contributions to four photon and four graviton scattering initiated in section 7. In particular it would be useful to convert the arguments presented in section 7 for the absence of exchange contributions to four graviton S-matrices consistent with the CRG bound into a tight mathematical proof for the same result.

Assuming the validity of the CRG conjecture, the analysis of this paper comes very near to establishing Conjectures 1 and 2 (see the introduction) for the case of four graviton scattering at least for $D \leq 6$. Note that the CRG conjecture is also violated by exchange contributions using the three point scattering amplitudes from the Gauss-Bonnet and three Riemann terms (see (4.20)). In other words the CRG conjecture, in addition to constraining four graviton scattering, also gives an alternate derivation of the results for three graviton scattering obtained in [2].

Apart from tightening up the arguments presented in this paper there are two major directions in which it would be interesting to move forward.

First it is unsatisfactory that progress towards establishing Conjectures 1 and 2 accomplished in this paper itself relies on a conjecture, namely the CRG conjecture. It would be very satisfying to prove this conjecture - and more generally to understand how once can systematically analyze the constraints of causality, positivity of energy, stability etc imposed on classical scattering matrices. We believe that such an analysis may not be too difficult to undertake. We hope to report on the results of such an analysis in future work.

Next, in this paper we have focused on the study of four point functions. In order to complete a classification of classical theories of gravity it is important that we are able to generalize our analysis to the study of 5 and higher point scattering amplitudes as well. Such a study might require a generalization of the CRG conjecture to higher point scattering, a result that would be easiest to obtain once (and if) we are able to prove the CRG conjecture for four particle scattering. The generalization of the analysis of this paper to the study of five and higher point gravitational scattering may also backreact on the study of four graviton scattering. Note, in this connection that even assuming the validity of the CRG conjecture we have not been able to demonstrate Conjectures 2 and 3 for four point scattering in $D \geq 7$. In these dimensions the Lagrangian (1.10) - when

\footnotetext{
${ }^{114}$ Somewhat unrelated to the main theme of the paper, our classification of polynomial S-matrices can be thought of the classification of counter-terms that contribute to four photon and four photon scattering. We thank R. Loganayagam for this observation.
} 
added to the Einstein Lagrangian - generates a four graviton S-matrix consistent with the CRG conjecture. It is conceivable, however, that the Lagrangian (1.10) is ruled out because it generates unacceptable contributions to 5 or higher graviton scattering, in much the same way that the Gauss-Bonnet coupling is ruled because of its effect of four graviton scattering. A generalization of the CRG conjecture to 5 (and higher) points would be needed to constrain 5 (and higher) point graviton scattering. An inelastic bound on chaos might be relevant for these considerations [43].

Through this paper we have focused our attention on S matrices in flat space. What implications do our results have for consistent classical asymptotically AdS solutions of gravity? It seems very likely to us that conjectures 2 and 3 have clear AdS analogues. The AdS analogue of conjecture 3 is the following claim. Consider any large $N$ CFT in which the stress tensor and its multitraces are closed under the OPE at leading nontrivial order in $1 / N$. The AdS analogue of conjecture 3 asserts that the leading order large $N$ stress tensor correlators in any such theory must be those generated by classical Einstein gravity in the bulk. It seems quite likely that this result is correct. It has already been demonstrated $[6,7]$ that the single trace stress tensor exchange contributions to four point functions in such theories necessarily have the structure that would follow from a dual bulk Einstein action (this is the AdS version of the causality results of [2]). As in the case of external scalars [23], the inversion formula [13] for stress tensor correlators at large $N$ should allow us to determine double trace exchange contributions ${ }^{115}$ to the same process almost uniquely in terms of single trace exchange. If it turns out that the words 'almost uniquely' in the previous sentence can be replaced by 'uniquely'116 then the AdS analogue of conjecture 3 would have been established at the level of 4 point functions. This will be the case provided that the four point function computed from any local bulk interaction violates the chaos bound; we expect this to be the case at least in $D \leq 6$ because of the close relationship between Regge growth and the chaos bound.

The AdS analogue of conjecture 2 would be the claim that large $N$ stress tensor correlators with a finite number of single trace exchange contributions are all also generated by the classical Einstein action in the bulk. ${ }^{117}$ At the level of 4 point functions, it may be possible to establish this conjecture by generalizing the arguments of $[6,7]$ to demonstrate that any non stress tensor single trace exchange contribution to the four stress tensor correlator (plus arbitrary double trace contributions to the same correlator) always violates causality (equivalently the chaos bound). It seems entirely possible to us that this result is true; this is already been studied to some extent in [24, 25].

We note that there is no simple AdS analogue of conjecture 1. Stress tensor correlators in $\mathcal{N}=4$ Yang Mills theory are nontrivial functions of the single continuous parameter $\lambda$. This lack of universality does not necessarily indicate a problem with conjecture 1 , but may instead have its roots in the limited nature of the universality on $R^{p} \times M_{10-p}$ postulated in

\footnotetext{
${ }^{115}$ And hence local bulk four graviton - roughly speaking Weyl ${ }^{4}$ interaction terms.

${ }^{116}$ I.e. if the 3 parameter ambiguity of the large $N$ scalar inversion formula listed in [23] is absent in the case of stress tensor four point functions.

${ }^{117}$ In other words there is no consistent theory in which the four point function of the stress tensor receives contributions from a finite number of single trace exchanges in addition to stress tensor exchange.
} 
conjecture 1 (universality applies only within a consistent truncation and not in solutions - like presumably AdS compactifications - that lie outside this truncation).

Returning to flat space, once several issues discussed in this section have been cleared up, and the status of Conjectures 2 and 3 is completely clarified, it may be possible to begin a meaningful study of the utterly fascinating possibility that string theory is the unique consistent classical extension of Einstein gravity, as effectively asserted in Conjecture 1. We leave these for future endeavors.

\section{Acknowledgments}

We would like to thank O. Aharony, N. Arkani Hamed, T. Dumitrescu, R. Gopakumar, T. Hartman, V. Hubeny, S. Caron-Huot, J. David, R. D. Koch, A. Maharana, J. Maldacena, G. Mandal, D. Meltzer, M. Mirbabayi, P. Nayak, K. Papadodimas, J. Penedones, R. Poojary, M. Rangamani, H. S. Reall, A. Rudra, A. Sen, A. Sever, A. Sinha, R. Sinha, R. Soni, D. Simmons-Duffin, D. Stanford, A. Strominger, S. Trivedi and A. Zhiboedov for very useful discussions. We would also like to thank J. David, T. Hartman, A. Maharana, P. Nayak, J. Penedones, R. Poojary, A. Rudra, A. Sen, D. Simmons-Duffin, A. Sinha, R. Soni, D. Stanford, S. Trivedi and A. Zhiboedov for comments on the manuscript. The work of all authors was supported by the Infosys Endowment for the study of the Quantum Structure of Spacetime. S.M. would like to thank ICTP Trieste for hospitality while this work was being carried out. The work of A.G. is also supported by SERB Ramanujan fellowship. A.G. would like to acknowledge that part of this work was performed at the Aspen Center for Physics, which is supported by National Science Foundation grant PHY-1607611. The work of T.G. is supported by DST Inspire fellowship. We would all also like to acknowledge our debt to the people of India for their steady support to the study of the basic sciences.

\section{A Discussion of conjecture 1}

\section{A.1 Universality of the consistent truncation}

The type II genus $g n$ graviton scattering amplitude on $R^{p} \times M_{10-p}$ is schematically given by the formula

$$
\mathcal{A}=\int d z d \tau\left\langle V_{1}\left(z_{1}\right) V_{2}\left(z_{2}\right) \ldots V_{n}\left(z_{n}\right)\right\rangle_{S_{\tau}}^{R^{p} \times M_{10-p}+\text { ghosts }}
$$

where $V_{i}$ is the vertex operator for the $i^{\text {th }}$ graviton, $z_{i}$ is the insertion point of this operator and the superscript of \langle\rangle denotes the CFT in which the expectation value is taken, and the subscript indicates the manifold on which this expectation is computed. $S_{\tau}$ is a genus $g$ Riemann surface with modulus $\tau$. The integral is over $\tau$ as well as (some of - i.e. the non fixed subset of) the $z_{i}$.

As graviton vertex operators lie entirely in the $R^{p}+$ ghosts part of the CFT (A.1) can be rewritten as

$$
\mathcal{A}=\int d \tau Z_{M_{10-p}}\left(S_{\tau}\right)\left(\int d z\left\langle V_{1}\left(z_{1}\right) V_{2}\left(z_{2}\right) \ldots V_{n}\left(z_{n}\right)\right\rangle_{S_{\tau}}^{R^{p}+\text { ghosts }}\right)
$$


where $Z_{M_{10-p}}\left(S_{\tau}\right)$ is the partition function on the Riemann surface $S_{\tau}$ of the sigma model on $M_{10-p}$. For $g \geq 1$ (A.1) is a detailed probe of the manifold $M_{10-p}$. In the special case $g=0$, however, the Riemann surface - a sphere - has no moduli so (A.2) further simplifies to

$$
\mathcal{A}=Z_{M_{10-p}}\left(S^{2}\right) \int d z\left\langle V_{1}\left(z_{1}\right) V_{2}\left(z_{2}\right) \ldots V_{n}\left(z_{n}\right)\right\rangle_{S^{2}}^{R^{p}+\text { ghosts }}
$$

Note that the only dependence of (A.3) on $M_{10-p}$ is through a single multiplicative constant $Z_{M_{10-p}}\left(S^{2}\right)$ which sets the effective value of the $p$ dimensional Newton constant.

The correlator that appears on the r.h.s. of (A.3) can, in principle, be computed as follows. We take the two $V_{i}\left(z_{i}\right)$ operators that are nearest to each other and use the OPE to expand the product of these two operators in a series of single operators. For each of the operators that appears in this series we can then once again replace the product of the two nearest $V_{i}$ with a sequence of other operators and so on. We can continue this process until the only operator insertion left is the identity. At this stage the correlator on the r.h.s. of (A.3) is a number equal to the $S^{2}$ partition function of the $R^{p}+$ ghost sigma model

As the $V_{i}$ are separately invariant under $(-1)^{F_{L}},(-1)^{F_{R}}$ and $\Omega$, all operators that appear on the r.h.s. of the OPEs described above also have this property. It follows from the discussion of the previous paragraph that the graviton scattering amplitude is the same in type IIA, type IIB and also in type I theory. Moreover it also follows that the exchange poles that appear in the relevant amplitudes all correspond to particles whose vertex operators are simultaneously invariant under $(-1)^{F_{L}},(-1)^{F_{R}}$ and $\Omega$.

\section{A.2 Warped compactifications}

The universality of graviton scattering amplitudes described in subsection A.1 was a consequence of the fact that the sigma model on $R_{p} \times M_{10-p}$ is the sum of two completely non interacting sigma models; namely the sigma model on $R_{p}$ and the sigma model on $M_{10-p}$. The argument of subsections A.1 does not go through for warped compactifications, i.e. compactifications on a spacetime of the schematic form

$$
d s^{2}=e^{\chi(y)} d x_{\mu}^{2}+g_{i j}(y) d y_{i} d y_{j}
$$

(here $x_{\mu}$ are coordinates on $R_{p}$ while $y_{i}$ are coordinates on $M_{10-p}$ ).

If it is possible to find consistent backgrounds of string theory of the form (A.4) in which $g_{s}$ can parametrically be taken to zero while the volume (and nature) of the compact manifold $M_{10-p}$ is held fixed then graviton scattering on such compactifications would likely deviate from type II or Heterotic versions of the Virasoro Shapiro amplitude. Such examples would thus violate conjecture 1 - and would be of great interest as they would generate new examples of consistent classical gravitational scattering amplitudes.

It is not, however, clear to us if examples of the form (A.4) do exist. In the rest of this subsection we provide a brief discussion of this point. ${ }^{118}$

One class of examples of warped compactifications is obtained by turning on RR fluxes (see e.g. [44]) on cycles of $M_{10-p}$. However the dilaton is often a fixed scalar in such

\footnotetext{
${ }^{118}$ We thank A. Maharana and S. Trivedi for very useful discussions on these issues.
} 
compactifications, and so cannot be taken to be parametrically small. When this is the case the corresponding flux compactifications do not contradict Conjecture 1 .

Models with a flat dilaton potential may be obtained by turning off fluxes but placing $R_{p}$ space filling branes and orientifold planes at points in $M_{10-p}$. In Type IIB theory with $p=4$, for instance, these branes would be $D 3$ branes. The total number of branes in such situations is fixed to be an order one number - by the requirement that the dilaton tadpole vanish (equivalently from the $D_{3}$ brane charge Gauss law). The dilaton potential is now completely flat and the dilaton can be made parametrically small. As the number of branes is held fixed while taking $g_{s} \rightarrow 0$, however, the cylinder, Klein bottle and Mobius strip contributions to $4 d$ gravity scattering amplitudes are a factor of $g_{s}$ smaller than the contribution of closed string tree level exchange ${ }^{119}$ and can be ignored in the $g_{s} \rightarrow 0$ limit. Thus graviton scattering amplitudes in this case once again reduce to the type II Virasoro Shapiro formula, and Conjecture 1 is not violated.

If $M_{10-p}$ is non-compact - e.g. is $R_{6}$ when $p=4$ - then there is no constraint on the number $N$ of $D 3$ branes. In this the limit $g_{s} \rightarrow 0, N \rightarrow \infty$ with $g_{s} N$ fixed, the contribution of open string loops to closed string scattering is of the same order as the contribution of closed string tree exchanges. As the AdS/CFT correspondence has emphasized, the dual description of these open string loop diagrams is summarized by closed string propagation a geometry that is non-trivially warped in the neighborhood of the $N$ branes. As $M_{10-p}$ is non-compact, however, in this example the function $\chi(y)$ (see (A.4)) is non-constant only on an infinitesimal fraction of the internal space. For this reason the $p$ dimensional gravitational $\mathrm{S}$ matrices in such a manifold are again given by the IIB Virasoro Shapiro amplitude. ${ }^{120}$ Once again conjecture 1 is not violated.

In summary, while it remains possible that the $g \rightarrow 0$ limit of compactifications of the form (A.4) exist and supply conterexaxmples to Conjecture 1, we have not been able to come up with a clear instance of such a counterexample.

\section{A.3 Constructing counter examples}

One could also try to come up with counter examples for conjecture 1 by simply guessing a gravity scattering amplitude that meets all consistency requirements. Such an attempt was made (for scalars), for instance, in [45]. Without the clear identification of a genuine string compactification that gives rise to the relevant amplitude, however, it would be difficult to be sure that a gravitational version of something like [45] is a genuine counter example to Conjecture 1. In order to establish this we would have to have an ansatz for all point scattering amplitudes of all the fields that lie within the minimal consistent truncation that contains gravity. This is, of course, a tall order, one that would be difficult to come up with. ${ }^{121,122}$

\footnotetext{
${ }^{119}$ Reflecting the fact that open strings, for instance, only run in loops in closed string scattering.

${ }^{120}$ Moreover with a value of Newton's constant that is strictly zero.

${ }^{121}$ In order to be at all convincing, an ansatz construction would at least need to specify the four particle scattering amplitudes external particles taken to be any of the fields in the minimum consistent truncation. This also sounds like a tall order.

${ }^{122}$ We thank J. Penedones, S. Trivedi and S. Zhiboedov for discussions on this point.
} 


\section{B Action of $S_{3}$ on functions of $(s, t, u)$}

In this section we study polynomials of $s, t$ and $u$ graded by their degree. We will be particularly interested in decomposing the space of such functions into distinct representations of the permutation group $S_{3}$ which permutes the three variables. We will perform our study both for unconstrained functions of $s, t$ and $u$, as well as for 'constrained' functions, i.e. functions that are regarded as identical if they agree when $s+t+u=0$. We start with a brief discussion of the permutation group $S_{3}$ and its representations.

\section{B.1 $S_{3}$ and its irreducible representations}

Let us first recall that the group $S_{3}$ has six elements. An element of the permutation group is said to be odd or even depending on whether it is built out of an odd or even number of permutations. We label an element of $S_{3}$ by the result of the action of that element on $(1,2,3)$, Thus $(1,2,3)$, the identity element $I$ is even. The other two even elements are the cyclical permutations $C=(2,3,1)$ and $C^{-1}=(3,1,2)$. The set of even elements of $S_{3}$ form the abelian subgroup $Z_{3}$. The odd elements of this group are the three permutations $P_{12}=(2,1,3), P_{13}=(3,2,1)$ and $P_{23}=(1,3,2)$.

If we think of 1,2 and 3 as basis elements of a three dimensional vector space then the action above yields a representation of $S_{3}$ in terms of $3 \times 3$ matrices. The representation is clearly reducible: all permutation elements act as identity on the basis vector $(1+2+3)$. This one dimensional representation is the completely symmetrical representation of $S_{3}$; this is the representation labelled by three boxes in the first row of the Young Tableaux.

On the other hand the two dimensional set of vectors with $1+2+3=0$ mix only among themselves under the permutation group, and so transform under a 2 dimensional representation of this group. A convenient basis for this space is found by diagonalizing $C$. Let

$$
B_{1}=e^{-\frac{2 \pi i}{3}}|1\rangle+|2\rangle+e^{\frac{2 \pi i}{3}}|3\rangle, \quad B_{2}=e^{\frac{2 \pi i}{3}}|1\rangle+|2\rangle+e^{-\frac{2 \pi i}{3}}|3\rangle
$$

Then

$$
C\left(\begin{array}{c}
B_{1} \\
B_{2}
\end{array}\right)=\left(\begin{array}{cc}
e^{-\frac{2 \pi i}{3}} & 0 \\
0 & e^{\frac{2 \pi i}{3}}
\end{array}\right)\left(\begin{array}{c}
B_{1} \\
B_{2}
\end{array}\right)
$$

The action of the permutations on the same basis is given by

$$
\begin{aligned}
P_{12}\left(\begin{array}{l}
B_{1} \\
B_{2}
\end{array}\right) & =\left(\begin{array}{cc}
0 & e^{-\frac{2 \pi i}{3}} \\
e^{\frac{2 \pi i}{3}} & 0
\end{array}\right)\left(\begin{array}{l}
B_{1} \\
B_{2}
\end{array}\right) \\
P_{23}\left(\begin{array}{l}
B_{1} \\
B_{2}
\end{array}\right) & =\left(\begin{array}{cc}
0 & e^{\frac{2 \pi i}{3}} \\
e^{\frac{-2 \pi i}{3}} & 0
\end{array}\right)\left(\begin{array}{l}
B_{1} \\
B_{2}
\end{array}\right) \\
P_{13}\left(\begin{array}{l}
B_{1} \\
B_{2}
\end{array}\right) & =\left(\begin{array}{ll}
0 & 1 \\
1 & 0
\end{array}\right)\left(\begin{array}{l}
B_{1} \\
B_{2}
\end{array}\right)
\end{aligned}
$$

Note that the phases that appear in the top right corner of the three matrices (B.3) are, respectively, $e^{-\frac{2 \pi i}{3}}, 1$ and $e^{\frac{2 \pi i}{3}}$. Of course the redefinition $B^{1} \rightarrow \alpha B^{1}$ changes each of these 
phases by $\alpha$. It follows that while the actual value of each phase is convention dependent, the ratios of the phases are physical (convention independent). We will encounter this fact below.

The equations (B.2) and (B.3) give a complete characterization of this two dimensional irreducible representation of $S_{3}$ (this is the representation labelled by the Young Tableaux with two boxes in its first row and one in its second row, or equivalently two boxes in the first column and one in the second column). From the fact that $P_{i j}^{2}=1$ it follows that the eigenvalues of the operator $P_{i j}= \pm 1$ in every representation of $S_{3}$. In this particular 2 dimensional representation it is easily verified that the two eigenvalues of $P_{i j}$ are plus one and minus one for every choice of $i$ and $j$.

Though it does not show up in the decomposition described above, there is a third irreducible representation of $S_{3}$. This is the completely antisymmetric representation labelled by a Young Tableaux with three boxes in the first column. In this one dimensional representation, every even element of the permutation element acts as unity (identity) while every odd element as -1 (negative identity).

\section{B.2 Left action of $S_{3}$ on itself}

Now consider the left action of $S_{3}$ on itself. This clearly generates a 6 dimensional reducible representation of the permutation group. Clearly the basis vector

$$
\begin{aligned}
& (123)+(231)+(312)+(213)+(321)+(132) \\
& I+S+S^{-1}+P_{12}+P_{13}+P_{23}
\end{aligned}
$$

transforms in the one dimensional symmetric representation. Similarly the basis vector

$$
\begin{aligned}
& (123)+(231)+(312)-(213)-(321)-(132) \\
& I+S+S^{-1}-P_{12}-P_{13}-P_{23}
\end{aligned}
$$

transforms in the one dimensional antisymmetric representation. What remains is the four dimensional vector space of elements

$$
A(123)+B(231)+C(312)+p(213)+q(132)+r(321)
$$

with $A+B+C=0$ and $p+q+r=0$. It is not difficult to decompose this four dimensional vector space into a direct sum of two copies of the two dimensional irreducible representation defined in the previous subsection. Define

$$
\begin{aligned}
& b_{1}^{ \pm}=e^{-\frac{2 \pi i}{3}}[(123) \pm(132)]+[(231) \pm(213)]+e^{\frac{2 \pi i}{3}}[(312) \pm(321)] \\
& b_{2}^{ \pm}=e^{\frac{2 \pi i}{3}}[(123) \pm(132)]+[(231) \pm(213)]+e^{-\frac{2 \pi i}{3}}[(312) \pm(321)] .
\end{aligned}
$$

With this definition it is easy to see that $b_{1,2}^{+}$and $b_{1,2}^{-}$transform in representation $\mathbf{2}_{\mathbf{M}}$.

\section{B.3 Clebsch-Gordon rules}

Let us use the symbols $\mathbf{1}_{\mathbf{S}}, \mathbf{1}_{\mathbf{A}}$ and $\mathbf{2}_{\mathbf{M}}$ to denote the symmetric, antisymmetric and 2 dimensional irreducible representations of $S_{3}$. Clearly

$$
\mathbf{1}_{\mathbf{S}} \times \mathbf{R}=\mathbf{R}
$$


where $\mathbf{R}$ denotes any of the irreps $\mathbf{1}_{\mathbf{S}}, \mathbf{1}_{\mathbf{A}}$ or $\mathbf{2}_{\mathbf{M}}$. On the other hand we have. ${ }^{123}$

$$
\mathbf{1}_{\mathrm{A}} \times \mathbf{1}_{\mathrm{S}}=\mathbf{1}_{\mathrm{A}}, \quad \mathbf{1}_{\mathrm{A}} \times \mathbf{1}_{\mathrm{A}}=\mathbf{1}_{\mathrm{S}}, \quad \mathbf{1}_{\mathrm{A}} \times \mathbf{2}_{\mathrm{M}}=\mathbf{2}_{\mathrm{M}}
$$

Finally we have

$$
2 \times 2_{M}=1_{S}+1_{A}+2_{M}
$$

To see how (B.9) works, let us use the tensor product vectors $B_{1} B_{1}, B_{1} B_{2}, B_{2} B_{1}$ and $B_{2} B_{2}$ as a basis for the four dimensional space $\mathbf{2} \times b f 2$. The vector

$$
B_{1} B_{2}+B_{2} B_{1}
$$

transforms in the singlet $\mathbf{1}_{\mathbf{S}}$ representation. The vector

$$
B_{1} B_{2}-B_{2} B_{1}
$$

transforms in the $\mathbf{1}_{\mathbf{A}}$ representation. Finally the action of $S_{3}$ on the doublet of vectors

$$
\left(\begin{array}{c}
B_{2} B_{2} \\
B_{1} B_{1}
\end{array}\right)
$$

is given precisely by the matrices listed in (B.2) and (B.3). It follows that this doublet of vectors transforms in the $\mathbf{2}_{\mathrm{M}}$ representation of $S_{3}$.

\section{B.4 Functions of 3 variables and the permutation group}

Consider a function of three variables $s, t$ and $u$. Let the permutation group $S_{3}$ act on these three variables. Given any particular function $f(s, t, u)$, the action of the permutation group generates up to 5 new functions.

If the original function was invariant under a subgroup of the permutation group then we would obtain fewer than 5 new functions. Let us first suppose that this is not the case. In this case the six dimensional linear vector space of the obtained functions transforms in a six dimensional representation of $S_{3}$. In fact the representation we find is identical to that of the previous subsection (representation of $S_{3}$ by left action on itself). So we obtain one copy of the symmetric representation, one copy of the antisymmetric representation and two copies of the two dimensional representation.

Consider a general function $f(s, t, u)$. Given any such function it is easy to break it up into a part that is completely symmetric, a part that is completely antisymmetric and

\footnotetext{
${ }^{123}$ The first two relations in (B.8) are obvious. In order to see the last relation note that $\mathbf{1}_{\mathbf{A}} \times \mathbf{2}_{\mathbf{M}}$ is a two dimensional vector space. Let $a$ be the vector that transforms in the $\mathbf{1}_{\mathbf{A}}$ and let $\left(B_{1}, B_{2}\right)$ be the doublet of vectors that transform under $S_{3}$ according to (B.2) and (B.3). It is easily verified that the doublet of tensor product vectors

$$
\left(\begin{array}{c}
-a B_{1} \\
a B_{2}
\end{array}\right)
$$

also transforms under the permutation group precisely as listed in (B.2) and (B.3). It follows that the two dimensional vector space $\mathbf{1}_{\mathrm{A}} \times \mathbf{2}_{\mathrm{M}}$ transforms in the $\mathbf{2}_{\mathrm{M}}$ representation of $S_{3}$.
} 
a part that lies somewhere in the (generically 4 dimensional) representation vector space of the two dimensional representations. We have

$$
\begin{aligned}
f(s, t, u) & =f^{\mathrm{sym}}(s, t, u)+f^{\text {as }}(s, t, u)+f^{\text {mixed }}(s, t, u) \\
f^{\mathrm{sym}}(s, t, u) & =P^{\mathrm{sym}} f=\frac{1}{6}(f(s, t, u)+f(t, u, s)+f(u, s, t)+f(t, s, u)+f(u, t, s)+f(s, u, t)) \\
f^{\text {as }}(s, t, u) & =P^{\text {as }} f=\frac{1}{6}(f(s, t, u)+f(t, u, s)+f(u, s, t)-f(t, s, u)-f(u, t, s)-f(s, u, t)) \\
f^{\text {mixed }}(s, t, u) & =P^{\text {mixed }} f=\frac{1}{3}(2 f(s, t, u)-f(t, u, s)-f(u, s, t))
\end{aligned}
$$

It is easy to verify that $P^{\mathrm{sym}}, P^{\text {as }}$ and $P^{\text {mixed }}$ all square to themselves and so are projectors. Moreover they project onto orthogonal subspaces, so that the product of two non equal projectors vanishes. Finally, these projectors commute with the action of the permutation group. The last equation in (B.13) asserts that the polynomials that transform in the mixed representations vanish under $\mathbb{Z}_{3}$ symmetrization as well as under complete symmetrization (these two facts imply these functions also vanish under complete anti-symmetrization).

\section{C $\quad \mathbb{Z}_{2} \times \mathbb{Z}_{2}$ invariance}

In this appendix we present a simple physics derivation of the formula (3.2).

Consider any 'single particle Hilbert space' with a basis single particle eigenstates $|i\rangle$ with definite values of the commuting charges $J_{m}$ and a single particle partition function

$$
\operatorname{Tr}_{\rho}\left(\prod_{m} y_{m}^{J_{m}}\right)=\sum_{i}\left\langle i\left|\prod_{m} y_{m}^{J_{m}}\right| i\right\rangle=z\left(y_{m}\right)
$$

Next consider the Hilbert space of two identical bosons/fermions, each of whose single particle Hilbert space is $\rho$. Let the corresponding Hilbert spaces be denoted by $S^{2} \rho$ and $\wedge^{2} \rho$ respectively. $S^{2} \rho$ and $\wedge^{2} \rho$ may, respectively, be thought of as the projection of the two (distinguishable) particle Hilbert spaces onto the subspaces on which the permutation operator $P_{(12)}$ (exchange of the two particles) has eigenvalue \pm 1 . It follows that

$$
\begin{aligned}
& \operatorname{Tr}_{S^{2} \rho}\left(\prod_{m} y_{m}^{J_{m}}\right)=\sum_{i_{1}, i_{2}}\left\langle i_{i} i_{2}\left|\left(\prod_{m} y_{m}^{J_{m}}\right)\left(\frac{1+P_{(12)}}{2}\right)\right| i_{1} i_{2}\right\rangle=\frac{z^{2}\left(y_{m}\right)+z\left(y_{m}^{2}\right)}{2} \\
& \operatorname{Tr}_{\Lambda^{2} \rho}\left(\prod_{m} y_{m}^{J_{m}}\right)=\sum_{i_{1}, i_{2}}\left\langle i_{i} i_{2}\left|\left(\prod_{m} y_{m}^{J_{m}}\right)\left(\frac{1-P_{(12)}}{2}\right)\right| i_{1} i_{2}\right\rangle=\frac{z^{2}\left(y_{m}\right)-z\left(y_{m}^{2}\right)}{2}
\end{aligned}
$$

where we have used the fact that

$$
\left\langle i_{1} i_{2}\left|\left(\prod_{m} y_{m}^{J_{m}}\right) P_{(12)}\right| i_{1} i_{2}\right\rangle=\left\langle i_{1} i_{2}\left|\left(\prod_{m} y_{m}^{J_{m}}\right)\right| i_{2} i_{1}\right\rangle=\delta_{i_{1}, i_{2}}\left\langle i_{1}\left|\left(\prod_{m}\left(y_{m}^{2}\right)^{J_{m}}\right)\right| i_{1}\right\rangle
$$

Next consider the Hilbert space $\rho$ of four distinguishable particles, each of whose single particle state space is spanned by $|i\rangle$. The partition function over this Hilbert space is, of 
course, given by

$$
\operatorname{Tr}_{\rho} \otimes 4\left(\prod_{m} y_{m}^{J_{m}}\right)=\sum_{i_{1}, i_{2}, i_{3}, i_{4}}\left\langle i_{i} i_{2} i_{3} i_{4}\left|\prod_{m} y_{m}^{J_{m}}\right| i_{1} i_{2} i_{3} i_{4}\right\rangle=z^{4}\left(y_{m}\right)
$$

Finally consider the same partition function but now over the four distinguishable particle Hilbert Space projected onto the subspace of $\mathbb{Z}_{2} \times \mathbb{Z}_{2}$ invariants, i.e. the space $\left.\rho^{\otimes 4}\right|_{\mathbb{Z}_{2} \times \mathbb{Z}_{2}}$. We find

$$
\begin{aligned}
& \operatorname{Tr}_{\rho}^{\otimes 4} \mid \mathbb{Z}_{2} \times \mathbb{Z}_{2} \\
&\left(\prod_{m} y_{m}^{J_{m}}\right)= \sum_{i_{1}, i_{2}, i_{3}, i_{4}}\left\langle i_{i} i_{2} i_{3} i_{4}\left|\left(\prod_{m} y_{m}^{J_{m}}\right)\left(\frac{1+P_{(2143)}+P_{(3412)}+P_{(4321)}}{4}\right)\right| i_{1} i_{2} i_{3} i_{4}\right\rangle \\
&= \frac{1}{4} \sum_{i_{1}, i_{2}, i_{3}, i_{4}}\left(\left\langle i_{1} i_{2} i_{3} i_{4}\left|\prod_{m} y_{m}^{J_{m}}\right| i_{1} i_{2} i_{3} i_{4}\right\rangle+\left\langle i_{1} i_{2} i_{3} i_{4}\left|\prod_{m} y_{m}^{J_{m}}\right| i_{2} i_{1} i_{4} i_{3}\right\rangle\right. \\
&\left.\quad+\left\langle i_{1} i_{2} i_{3} i_{4}\left|\prod_{m} y_{m}^{J_{m}}\right| i_{3} i_{4} i_{1} i_{2}\right\rangle+\left\langle i_{1} i_{2} i_{3} i_{4}\left|\prod_{m} y_{m}^{J_{m}}\right| i_{4} i_{3} i_{2} i_{1}\right\rangle\right) \\
&= \frac{z^{4}\left(y_{m}\right)+3 z^{2}\left(y_{m}^{2}\right)}{4} \\
&= z^{4}\left(y_{m}\right)-3\left(\frac{z^{2}\left(y_{m}\right)+z\left(y_{m}^{2}\right)}{2}\right) \times\left(\frac{z^{2}\left(y_{m}\right)-z\left(y_{m}^{2}\right)}{2}\right)
\end{aligned}
$$

(In going from the second to the third line of (C.5) we have used manipulations similar to (C.3).)

Comparing (C.5), (C.4) and (C.2) we conclude that

$$
\left.\operatorname{Tr}_{\rho \otimes 4}\right|_{\mathbb{Z}_{2} \times \mathbb{Z}_{2}}\left(\prod_{m} y_{m}^{J_{m}}\right)=\operatorname{Tr}_{\rho \otimes 4}\left(\prod_{m} y_{m}^{J_{m}}\right)-3 \operatorname{Tr}_{S^{2} \rho}\left(\prod_{m} y_{m}^{J_{m}}\right) \operatorname{Tr}_{\Lambda^{2} \rho}\left(\prod_{m} y_{m}^{J_{m}}\right)
$$

an equation that can schematically be written in the form (3.2) i.e.

$$
\left.\rho^{\otimes 4}\right|_{\mathbb{Z}_{2} \times \mathbb{Z}_{2}}=\rho^{\otimes 4}-3 S^{2} \rho \otimes \Lambda^{2} \rho
$$

In the context of the discussion around (3.2) the single particle Hilbert Space $\rho$ is the space of photon or graviton polarizations. $J_{m}$ are the $\mathrm{SO}(D-3)$ Cartan charges. The projection onto $\mathrm{SO}(D-3)$ singlets could be achieved by integrating (C.6) over the $\mathrm{SO}(D-3)$ Haar measure.

\section{Bare index structures in low dimensions}

In this appendix we will complete the discussion of subsection 3.2 by constructing parity even and parity odd generators of the bare module for photons (called $e$ 's and o's respectively) and for gravitons for $D<7$. 


\section{D.1 Photons}

$\boldsymbol{D}=\mathbf{6}$. In this case, the parity even bare structures are the same as those in i.e $D \geq 7$ (i.e. continue to be given by the construction depicted in figure 1 and so continue to transform in the representations listed in (3.7)). The parity odd structure which transforms in the $\mathbf{1}_{\mathbf{A}}$ representation. This structure is simply

$$
o_{\mathbf{A}}^{D=6}=\left.N\left(\widetilde{\varepsilon}^{3}\right)_{\mu \nu \rho} \epsilon_{1}^{\perp \mu} \epsilon_{2}^{\perp \nu} \epsilon_{3}^{\perp \rho}\right|_{\mathbb{Z}_{2} \times \mathbb{Z}_{2}}=\left.\frac{\varepsilon_{\alpha \beta \gamma \mu \nu \rho} p_{1}^{\alpha} p_{2}^{\beta} p_{3}^{\gamma}}{\sqrt{s t u}} \epsilon_{1}^{\perp \mu} \epsilon_{2}^{\perp \nu} \epsilon_{3}^{\perp \rho}\right|_{\mathbb{Z}_{2} \times \mathbb{Z}_{2}} .
$$

Writing out the sum over $\mathbb{Z}_{2} \times \mathbb{Z}_{2}$ orbits explicitly (and using $p_{4}=-p_{1}-p_{2}-p_{3}$ to simplify the expressions), this structure is proportional to

$$
\begin{aligned}
o_{\mathbf{A}}^{D=6}= & \frac{\varepsilon_{\alpha \beta \gamma \mu \nu \rho} p_{1}^{\alpha} p_{2}^{\beta} p_{3}^{\gamma}}{\sqrt{s t u}} \\
& \times\left(\epsilon_{1}^{\perp \mu} \epsilon_{2}^{\perp}{ }^{\perp} \epsilon_{3}^{\perp} \alpha_{4}+\epsilon_{2}^{\perp}{ }^{\mu} \epsilon_{1}^{\perp \nu} \epsilon_{4}^{\perp} \rho \alpha_{3}+\epsilon_{3}^{\perp} \epsilon_{4}^{\perp \nu} \epsilon_{1}^{\perp} \rho \alpha_{2}+\epsilon_{4}^{\perp} \epsilon_{3}^{\perp \nu} \epsilon_{2}^{\perp} \rho \alpha_{1}\right)
\end{aligned}
$$

According to the rules spelt out in subsubsection 2.2.2, (D.2) transforms in the completely antisymmetric representation of $S_{4}$ (and so, in particular, in the $\mathbf{1}_{\mathbf{A}}$ representation $S_{3}=$ $S_{4} /\left(\mathbb{Z}_{2} \times \mathbb{Z}_{2}\right)$.

$\boldsymbol{D}=\mathbf{5}$. As above, the parity even bare structures are the same as those in $D \geq 7$ (i.e. continue to be given by the construction depicted in figure 1 and so continue to transform in the representations listed in (3.7)).

We now turn to parity odd structures. A priori, one could have tried to make parity odd structures using $\widetilde{\varepsilon}_{\mu \nu}^{2}$. We need to be either in subsector $\mathrm{v}^{\otimes 4}$ or $\mathrm{v}^{\otimes 2} \mathrm{~s}^{\otimes 2}$. We consider these two cases in turn.

In the first case, when we contract one pair of vectors using $\widetilde{\varepsilon}$, the other needs to be contracted with Kronecker $\delta$. An example of such a term is the $Z_{2} \times \mathbb{Z}_{2}$ symmetrized version of

$$
\left(\widetilde{\varepsilon}_{\mu \nu}^{2} \epsilon_{1}^{\perp} \mu \epsilon_{2}^{\perp \nu}\right)\left(\epsilon_{3}^{\perp} . \epsilon_{4}^{\perp}\right)
$$

Explicitly performing the $\mathbb{Z}_{2} \times \mathbb{Z}_{2}$ of this term we obtain

$$
\widetilde{\varepsilon}_{\mu \nu}^{2}\left(\epsilon_{1}^{\perp} \epsilon_{2}^{\perp \nu}\left(\epsilon_{3}^{\perp} \cdot \epsilon_{4}^{\perp}\right)+\epsilon_{2}^{\perp \mu} \epsilon_{1}^{\perp \nu}\left(\epsilon_{4}^{\perp} \cdot \epsilon_{3}^{\perp}\right)+\epsilon_{3}^{\perp} \mu \epsilon_{4}^{\perp \nu}\left(\epsilon_{1}^{\perp} \cdot \epsilon_{2}^{\perp}\right)+\epsilon_{4}^{\perp \mu} \epsilon_{3}^{\perp \nu}\left(\epsilon_{2}^{\perp} \cdot \epsilon_{1}^{\perp}\right)\right)=0
$$

Consequently there are no $\mathbb{Z}_{2} \times \mathbb{Z}_{2}$ symmetric expressions in $\mathrm{v}^{\otimes 4}$.

The analysis for the second case is very similar. One can write down singlets in the sector $\mathrm{v}^{\otimes 2} \mathbf{s}^{\otimes 2}$ : for example

$$
\left(\widetilde{\varepsilon}_{\mu \nu}^{2} \epsilon_{1}^{\perp \mu} \epsilon_{2}^{\perp \nu} \alpha_{3} \alpha_{4}\right)
$$

$\mathbb{Z}_{2} \times \mathbb{Z}_{2}$ symmetrization kills these structures - the algebra that demonstrates this is very similar to (D.3).

It follows that there are no parity odd photon scattering structures in $D=5$. 
$\boldsymbol{D}=\mathbf{4}$. In this case, there is a reduction in the number of parity even structures compared to the higher dimensions and also there are two parity odd structures. Let us first discuss the parity even case. In $D=4, \epsilon_{i}^{\perp}$ are vectors of $\mathrm{SO}(1)$, they can simply be thought of as numbers. Given this, the three states of $e_{\mathbf{3}, 1}$,

$$
\left(\epsilon_{1}^{\perp} \cdot \epsilon_{2}^{\perp}\right)\left(\epsilon_{3}^{\perp} \cdot \epsilon_{4}^{\perp}\right), \quad\left(\epsilon_{2}^{\perp} \cdot \epsilon_{3}^{\perp}\right)\left(\epsilon_{1}^{\perp} \cdot \epsilon_{4}^{\perp}\right) \quad\left(\epsilon_{3}^{\perp} \cdot \epsilon_{1}^{\perp}\right)\left(\epsilon_{2}^{\perp} \cdot \epsilon_{4}^{\perp}\right)
$$

are now indistinguishable from each other and so transform in the $\mathbf{1}_{\mathbf{S}}$ representation. We denote this structure as $e_{\mathbf{3} \rightarrow \mathbf{S}}$.

On the other hand the structure $e_{\mathbf{3}, 2}$, which transformed in the $\mathbf{3}$ for $D \geq 5$, continue to transform in $\mathbf{3}$ even in $D=4$. Similarly the symmetric structure $e_{\mathbf{S}}$ continues to transform in the $\mathbf{1}_{\mathbf{S}}$ in $D=4$ as it did for $D \geq 5$. In summary, the parity even bare photon structures in $D=4$ transform in the

$$
\mathbf{3}+2 \cdot \mathbf{1}_{\mathbf{S}}
$$

We now turn to parity odd structures. All such structures lie either in the vs ${ }^{\otimes 3}$ or in the $\mathrm{v}^{\otimes 3} \mathrm{~s}$ and are given by the $\mathbb{Z}_{2} \times \mathbb{Z}_{2}$ symmetrization

$$
o_{\mathbf{S}, 1}^{D=4}=\left.\left(N(\widetilde{\varepsilon})_{\mu} \epsilon_{4 \mu}^{\perp}\right) \alpha_{1} \alpha_{2} \alpha_{3}\right|_{\mathbb{Z} 2 \times \mathbb{Z} 2} \quad \text { and } \quad o_{\mathbf{S}, 2}^{D=4}=\left(N(\widetilde{\varepsilon})_{\mu} \epsilon_{4 \mu}^{\perp}\right) \epsilon_{1 \nu}^{\perp} \epsilon_{2 \nu}^{\perp} \alpha_{3} \|_{\mathbb{Z} 2 \times \mathbb{Z} 2 .} .
$$

Explicitly performing the $\mathbb{Z}_{2} \times \mathbb{Z}_{2}$ symmetrization we obtain

$$
\begin{aligned}
& o_{\mathbf{S}, 1}^{D=4}=N(\widetilde{\varepsilon})_{\mu}\left(\epsilon_{1}^{\perp \mu} \alpha_{2} \alpha_{3} \alpha_{4}+\epsilon_{2}^{\perp} \alpha_{1} \alpha_{3} \alpha_{4}+\epsilon_{3}^{\perp \mu} \alpha_{1} \alpha_{2} \alpha_{4}+\epsilon_{4}^{\perp \mu} \alpha_{1} \alpha_{2} \alpha_{3}\right) \\
& o_{\mathbf{S}, 2}^{D=4}=N(\widetilde{\varepsilon})_{\mu}\left(\epsilon_{3}^{\perp} \epsilon_{1}^{\perp \nu} \epsilon_{2}^{\perp \nu} \alpha_{4}+\epsilon_{1}^{\perp} \epsilon_{4}^{\perp} \nu \epsilon_{3}^{\perp \nu} \alpha_{2}+\epsilon_{2}^{\perp} \epsilon_{3}^{\perp}{ }^{\perp} \epsilon_{4}^{\perp \nu} \alpha_{1}+\epsilon_{4}^{\perp} \epsilon_{1}^{\perp \nu} \epsilon_{2}^{\perp}{ }^{\perp} \alpha_{3}\right)
\end{aligned}
$$

The term in the first line of (D.7) is manifestly an $S_{4}$ (and so an $S_{3}$ ) singlet. Though it is less manifest, the same is also true of the term in the second line of (D.7). This result follows upon using the identity

$$
\epsilon_{i}^{\perp \nu} \epsilon_{j}^{\perp \nu} \epsilon_{k}^{\perp} \mu=\epsilon_{j}^{\perp \mu} \epsilon_{i}^{\perp \nu} \epsilon_{k}^{\perp \nu}
$$

((D.8) holds because $\epsilon_{i}^{\perp}$ all point along the same direction in $\left.D=4\right)$. Using the rules of subsubsection 2.2.2 the $D=4$ parity odd structures transform in the

$$
2 \cdot \mathbf{1}_{\mathrm{S}}
$$

$\boldsymbol{D}=3$. In this case, $\epsilon_{i}^{\perp}$ does not exist. The S-matrix for photons becomes the same as the S-matrix for scalars. For scalars there is a single parity even structure as well as a single parity odd structure. The parity even and parity odd structures are,

$$
e_{\mathbf{S}}=1, \quad o_{\mathbf{A}}^{D=3}=\varepsilon_{\alpha \beta \gamma} p_{1}^{\alpha} p_{2}^{\beta} p_{3}^{\gamma} .
$$

respectively. Remember that they have implicit factors of $\alpha_{1} \alpha_{2} \alpha_{3} \alpha_{4}$. 


\section{D.2 Gravitons}

$\boldsymbol{D}=\mathbf{6}$. The parity even structures for $D \geq 7$ were all constructed and diagrammatically depicted in figure 2. The structures depicted in figure 2 transformed in the representations listed in (3.8) which we reproduce for convenience

$$
29=10 \cdot \mathbf{1}_{\mathbf{S}}+9 \cdot \mathbf{2}_{\mathbf{M}}+1 \cdot \mathbf{1}_{\mathbf{A}} .
$$

The parity even structures in $D=6$ differ from those in $D \geq 7$ in only one respect. The equation

$$
\left(\epsilon_{1}^{\perp} \wedge \epsilon_{2}^{\perp} \wedge \epsilon_{3}^{\perp} \wedge \epsilon_{4}^{\perp}\right)^{2}=0 .
$$

holds in $D=6$ (though not for $D \geq 7$ ) because the four transverse polarizations lie in only three dimensions hence their anti-symmetrized product vanishes. The equation (D.12) is parity even and clearly transforms in the $\mathbf{1}_{\mathbf{S}}$ representation. Since the equation involved terms with eight factors of $\epsilon^{\perp}$, it follows that (D.12) implies that a particular linear combination of the $\mathbf{1}_{\mathrm{S}}$ part of subfigures 1 ) and 2) in figure 7 vanish. It follows that the parity even structures in $D=6$ have one less $\mathbf{1}_{\mathbf{S}}$ structure than their counterpart in $D \geq 7$. In summary, the parity even bare structures in 6 dimensions transform in the

$$
28=9 \cdot \mathbf{1}_{\mathbf{S}}+9 \cdot \mathbf{2}_{\mathbf{M}}+1 \cdot \mathbf{1}_{\mathbf{A}} .
$$

As the $\widetilde{\varepsilon}$ tensor has 3 free indices, parity odd structures potentially appear in sectors with 3,5 or 7 free indices (we need at least 3 free indices to saturate those in $\widetilde{\varepsilon}$ and the rest can contract in pairs), i.e the subsectors $\mathrm{v}^{\otimes 3} \mathrm{~s}, \mathrm{tv} \mathrm{v}^{\otimes 3}, \mathrm{t}^{\otimes 2} \mathrm{vs}$ and $\mathrm{t}^{\otimes 3} \mathrm{v}$. Note also that all structures that appear in each of these sectors are necessarily parity odd (the odd number of indices can only all contract if there is a free $\widetilde{\varepsilon}$ ).

We take up these subsectors in turn. We will first count the number of $\mathrm{SO}(3) \times\left(\mathbb{Z}_{2} \times \mathbb{Z}_{2}\right)$ invariants in each sector separately and then explicitly construct these structures in order to deduce their $S_{3}$ representation counting.

In order to count the structures we proceed along the lines of appendix C. To count the singlets in any given sector we evaluate a trace of the sort listed in the first of (C.5). In this trace we allow the indices $i_{1}, i_{2}, i_{3}, i_{4}$ to range over all values constrained by the net representation content. For instance in the channel $t^{\otimes 2} \mathrm{vs}$ two of the four indices $i_{1}, i_{2}, i_{3}, i_{4}$ should range over the polarizations of the tensor, one of them should range over the polarizations of the vector while the last one should be the (unique) polarization of the scalar. One possibility is that $i_{1}$ is the scalar, $i_{2}$ is the vector and $i_{3}$ and $i_{4}$ range over values for the tensor. Of course the $i s$ can be distributed between representations in different ways. In this sector the total number of ways in which this assignment can be made is $4 \times 3=12$. In each of the other three sectors namely $\mathrm{v}^{\otimes 3} \mathrm{~s}, \mathrm{tv}^{\otimes 3}$ and $\mathrm{t}^{\otimes 3} \mathrm{v}$, it is easy to check that the $i$ 's can be distributed between representations in 4 ways. Each of the different ways of assignment runs in the trace in the first line of (C.5).

Now the first line of (C.5) involves inner products of states sandwiched with a $\mathbb{Z}_{2} \times \mathbb{Z}_{2}$ permutation operator. It is easy to verify that no distribution of $i$ 's among available representations is left invariant by any $\mathbb{Z}_{2} \times \mathbb{Z}_{2}$ permutation; this is true for each choice of sectors. 


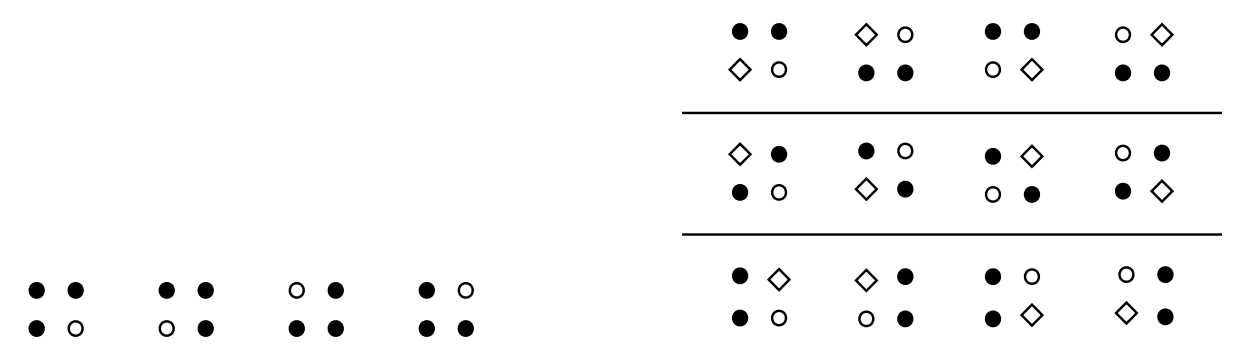

Figure 4. The left figure shows that there is a single $\mathbb{Z}_{2} \times \mathbb{Z}_{2}$ orbit in subsectors $\mathrm{t}^{\otimes 3} \mathrm{v}, \mathrm{tv} \mathrm{v}^{\otimes 3}, \mathrm{v}^{\otimes 3} \mathrm{~s}$. The right figure shows the three distinct $\mathbb{Z}_{2} \times \mathbb{Z}_{2}$ orbits in the subsector $\mathrm{t}^{\otimes 2} \mathrm{vs}$.

It follows that every matrix element involving a non-trivial permutation element in the first line of (C.5) vanishes. The only matrix elements that do not vanish are those involving the identity operator. It follows that the r.h.s. of the first line of (C.5) can be replaced by

$$
A \sum_{i_{1}, i_{2}, i_{3}, i_{4}}\left\langle i_{i} i_{2} i_{3} i_{4}\left|\left(\prod_{m} y_{m}^{J_{m}}\right)\right| i_{1} i_{2} i_{3} i_{4}\right\rangle
$$

where $i_{1}, i_{2}, i_{3}, i_{4}$ are assigned to the representations in any given particular way and the number $A$ is the total number of ways of making this assignment divided by 4 . In other words $A=3$ in the $\mathrm{t}^{\otimes 2} \mathrm{vs}$ sector but is equal to unity in the $\mathrm{v}^{\otimes 3} \mathrm{~s}, \mathrm{tv}^{\otimes 3}$ and $\mathrm{t}^{\otimes 3} \mathrm{v}$ sectors.

We thus conclude that the total number of simultaneous $\mathrm{SO}(3)$ and $\mathbb{Z}_{2} \times \mathbb{Z}_{2}$ singlets in any given sector is simply $A$ times the number of $\mathrm{SO}(3)$ singlets in the Clebsch-Gordon decomposition of the corresponding representations (the CG decomposition is done without imposing any symmetry constraints). The procedure described above has a generalization to every $D .{ }^{124}$ The case $D=6$ is particularly simple as in this case $\mathrm{t}, \mathrm{v}, \mathrm{s}$ are simply spin 2 , spin 1 and spin 0 representations of $\mathrm{SO}(3)$ and their fusion rules are very familiar.

There is another more combinatorial way of understanding the conclusion of the last paragraph. For each of the subsectors $\mathrm{t}^{\otimes 3} \mathrm{v}, \mathrm{tv}^{\otimes 3}, \mathrm{v}^{\otimes 3} \mathrm{~s}$ all assignments of $i s$ to representations are related by $\mathbb{Z}_{2} \times \mathbb{Z}_{2}$ transforms. In the subsector $t^{\otimes 2} \mathrm{vs}$, distinct assignments of $i s$ to representations lie in three distinct $\mathbb{Z}_{2} \times \mathbb{Z}_{2}$ orbits. This is explained graphically in figure 4 . This is why number of simultaneous $\mathrm{SO}(3)$ and $\mathbb{Z}_{2} \times \mathbb{Z}_{2}$ singlets in $\mathrm{t}^{\otimes 2}$ vs is thrice the number of $\mathrm{CG}$ singlets.

We now consider the sectors one at a time. Consider $\mathrm{v}^{\otimes 3} \mathrm{~s}$. The number of $\mathrm{CG}$ singlets in the product of 3 vectors is unity. As $A=1$ we have a single structure. It is easy to construct the corresponding structure; it is given by

$$
\begin{aligned}
N\left(\widetilde{\varepsilon}^{3}\right)_{\mu \nu \rho} & \left(\epsilon_{1}^{\perp \mu} \epsilon_{2}^{\perp \nu} \epsilon_{3}^{\perp} \rho \alpha_{1} \alpha_{2} \alpha_{3} \alpha_{4}^{2}+\epsilon_{2}^{\perp \mu} \epsilon_{1}^{\perp \nu} \epsilon_{4}^{\perp} \rho_{\alpha_{2}} \alpha_{1} \alpha_{4} \alpha_{3}^{2}+\epsilon_{3}^{\perp \mu} \epsilon_{4}^{\perp \nu} \epsilon_{1}^{\perp} \rho_{\alpha_{3}} \alpha_{4} \alpha_{1} \alpha_{2}^{2}\right. \\
& \left.+\epsilon_{4}^{\perp \mu} \epsilon_{3}^{\perp}{ }^{\nu} \epsilon_{2}^{\perp}{ }^{\rho} \alpha_{4} \alpha_{3} \alpha_{2} \alpha_{1}^{2}\right) .
\end{aligned}
$$

\footnotetext{
${ }^{124}$ When $D$ is odd, however, the separation into parity even and parity odd structures is more difficult for the bare module as $\widetilde{\varepsilon}$ then has an even number of free indices.
} 
According to the rules of subsubsection 2.2.2 this structure transforms in the $\mathbf{1}_{\mathbf{A}}$ representation.

Next let us turn to the $\mathrm{t}^{\otimes 3} \mathrm{v}$. Once again in this case $A=1$ and the number of CG singlets is 3 so we have 3 structures. These structures are easily constructed; they are given by

$$
\begin{aligned}
& N\left(\widetilde{\varepsilon}^{3}\right)_{\mu \nu \rho}\left(\epsilon_{1}^{\perp \mu} \epsilon_{2}^{\perp \nu} \epsilon_{3}^{\perp \rho} \alpha_{4}+\epsilon_{2}^{\perp \mu} \epsilon_{1}^{\perp \nu} \epsilon_{4}^{\perp \rho} \alpha_{3}+\epsilon_{3}^{\perp \mu} \epsilon_{4}^{\perp \nu} \epsilon_{1}^{\perp \rho} \alpha_{2}+\epsilon_{4}^{\perp \mu} \epsilon_{3}^{\perp \nu} \epsilon_{2}^{\perp} \rho \alpha_{1}\right)\left(\epsilon_{1}^{\perp} . \epsilon_{2}^{\perp} \epsilon_{3}^{\perp} . \epsilon_{4}^{\perp}\right), \\
& N\left(\widetilde{\varepsilon}^{3}\right)_{\mu \nu \rho}\left(\epsilon_{1}^{\perp \mu} \epsilon_{3}^{\perp \nu} \epsilon_{2}^{\perp \rho} \alpha_{4}+\epsilon_{3}^{\perp \mu} \epsilon_{1}^{\perp \nu} \epsilon_{4}^{\perp \rho} \alpha_{2}+\epsilon_{2}^{\perp \mu} \epsilon_{4}^{\perp \nu} \epsilon_{1}^{\perp \rho} \alpha_{3}+\epsilon_{4}^{\perp \mu} \epsilon_{2}^{\perp \nu} \epsilon_{3}^{\perp \rho} \alpha_{1}\right)\left(\epsilon_{1}^{\perp} \cdot \epsilon_{3}^{\perp} \epsilon_{2}^{\perp} . \epsilon_{4}^{\perp}\right), \\
& N\left(\widetilde{\varepsilon}^{3}\right)_{\mu \nu \rho}\left(\epsilon_{1}^{\perp \mu} \epsilon_{4}^{\perp \nu} \epsilon_{3}^{\perp \rho} \alpha_{2}+\epsilon_{4}^{\perp \mu} \epsilon_{1}^{\perp \nu} \epsilon_{2}^{\perp \rho} \alpha_{3}+\epsilon_{3}^{\perp \mu} \epsilon_{2}^{\perp \nu} \epsilon_{1}^{\perp \rho} \alpha_{4}+\epsilon_{2}^{\perp \mu} \epsilon_{3}^{\perp \nu} \epsilon_{4}^{\perp \rho} \alpha_{1}\right)\left(\epsilon_{1}^{\perp} . \epsilon_{4}^{\perp} \epsilon_{3}^{\perp} . \epsilon_{2}^{\perp}\right)
\end{aligned}
$$

Using the rules of subsubsection 2.2.2 the three structures in (D.2) clearly transform in $\mathbf{3}_{\mathbf{A}}$.

In $\mathrm{t}^{\otimes 2}$ vs we have $1 \mathrm{CG}$ singlet but $A=3$ and so we have three structures. These structures transform in the $\mathbf{3}_{\mathbf{A}}$ representation and are given by

$$
\begin{aligned}
& N\left(\widetilde{\varepsilon}^{3}\right)_{\mu \nu \rho}\left(\left(\epsilon_{1}^{\perp} \epsilon_{2}^{\perp \nu} \epsilon_{3}^{\perp \rho} \alpha_{4}^{2} \alpha_{3}+\epsilon_{2}^{\perp} \mu \epsilon_{1}^{\perp \nu} \epsilon_{4}^{\perp} \rho \alpha_{3}^{2} \alpha_{4}\right) \epsilon_{1}^{\perp} . \epsilon_{2}^{\perp}\right. \\
& \left.+\left(\epsilon_{3}^{\perp}{ }^{\mu} \epsilon_{4}^{\perp \nu} \epsilon_{1}^{\perp} \rho \alpha_{2}^{2} \alpha_{1}+\epsilon_{4}^{\perp} \mu \epsilon_{3}^{\perp \nu} \epsilon_{2}^{\perp} \rho \alpha_{1}^{2} \alpha_{2}\right) \epsilon_{3}^{\perp} . \epsilon_{4}^{\perp}\right), \\
& N\left(\widetilde{\varepsilon}^{3}\right)_{\mu \nu \rho}\left(\left(\epsilon_{1}^{\perp} \epsilon_{3}^{\perp \nu} \epsilon_{2}^{\perp \rho} \alpha_{4}^{2} \alpha_{2}+\epsilon_{3}^{\perp \mu} \epsilon_{1}^{\perp \nu} \epsilon_{4}^{\perp} \rho \alpha_{2}^{2} \alpha_{4}\right) \epsilon_{1}^{\perp} . \epsilon_{3}^{\perp}\right. \\
& \left.+\left(\epsilon_{2}^{\perp} \mu \epsilon_{4}^{\perp \nu} \epsilon_{1}^{\perp} \rho \alpha_{3}^{2} \alpha_{1}+\epsilon_{4}^{\perp} \mu \epsilon_{2}^{\perp \nu} \epsilon_{3}^{\perp} \rho \alpha_{1}^{2} \alpha_{3}\right) \epsilon_{2}^{\perp} . \epsilon_{4}^{\perp}\right), \\
& N\left(\widetilde{\varepsilon}^{3}\right)_{\mu \nu \rho}\left(\left(\epsilon_{1}^{\perp} \epsilon_{4}^{\perp \nu} \epsilon_{3}^{\perp} \rho \alpha_{2}^{2} \alpha_{3}+\epsilon_{4}^{\perp \mu} \epsilon_{1}^{\perp \nu} \epsilon_{2}^{\perp} \rho \alpha_{3}^{2} \alpha_{2}\right) \epsilon_{1}^{\perp} . \epsilon_{4}^{\perp}\right. \\
& \left.+\left(\epsilon_{3}^{\perp} \epsilon_{2}^{\perp \nu} \epsilon_{1}^{\perp \rho} \rho \alpha_{4}^{2} \alpha_{1}+\epsilon_{2}^{\perp} \mu \epsilon_{3}^{\perp \nu} \epsilon_{4}^{\perp} \rho \alpha_{1}^{2} \alpha_{4}\right) \epsilon_{3}^{\perp} . \epsilon_{2}^{\perp}\right),
\end{aligned}
$$

Turning to the $\mathrm{tv}^{\otimes 3}$ sector, $A=1$ and there are $2 \mathrm{CG}$ singlets so we have 2 structures. As it is clear that there is no $t$ in the totally symmetric or anti-symmetric cube of $\mathrm{v}$, these 2 structures necessarily transform in $\mathbf{2}_{\mathbf{M}}$ representation. Explicitly they take the form,

$$
\begin{aligned}
& N\left(\widetilde{\varepsilon}^{3}\right)_{\mu \nu \rho}\left(\left(\epsilon_{1}^{\perp \mu} \epsilon_{4}^{\perp \nu} \epsilon_{3}^{\perp} \rho \alpha_{4} \alpha_{3} \alpha_{2}+\epsilon_{2}^{\perp} \mu \epsilon_{3}^{\perp} \epsilon_{4}^{\perp} \rho \alpha_{3} \alpha_{4} \alpha_{1}\right) \epsilon_{1}^{\perp} . \epsilon_{2}^{\perp}\right. \\
& \left.+\left(\epsilon_{4}^{\perp \mu} \epsilon_{1}^{\perp \nu} \epsilon_{2}^{\perp \rho} \rho \alpha_{3} \alpha_{2} \alpha_{1}+\epsilon_{3}^{\perp \mu} \epsilon_{2}^{\perp \nu} \epsilon_{1}^{\perp \rho} \alpha_{2} \alpha_{1} \alpha_{4}\right) \epsilon_{3}^{\perp} . \epsilon_{4}^{\perp}\right), \\
& N\left(\widetilde{\varepsilon}^{3}\right)_{\mu \nu \rho}\left(\left(\epsilon_{1}^{\perp \mu} \epsilon_{4}^{\perp \nu} \epsilon_{2}^{\perp} \rho \alpha_{4} \alpha_{2} \alpha_{3}+\epsilon_{3}^{\perp} \mu \epsilon_{2}^{\perp \nu} \epsilon_{4}^{\perp \rho} \alpha_{2} \alpha_{4} \alpha_{1}\right) \epsilon_{1}^{\perp} . \epsilon_{3}^{\perp}\right. \\
& \left.+\left(\epsilon_{4}^{\perp} \mu \epsilon_{1}^{\perp \nu} \epsilon_{3}^{\perp} \rho \alpha_{2} \alpha_{3} \alpha_{1}+\epsilon_{2}^{\perp} \mu \epsilon_{3}^{\perp \nu} \epsilon_{1}^{\perp \rho} \alpha_{3} \alpha_{1} \alpha_{4}\right) \epsilon_{4}^{\perp} . \epsilon_{2}^{\perp}\right), \\
& N\left(\widetilde{\varepsilon}^{3}\right)_{\mu \nu \rho}\left(\left(\epsilon_{1}^{\perp \mu} \epsilon_{2}^{\perp \nu} \epsilon_{3}^{\perp} \rho \alpha_{2} \alpha_{3} \alpha_{4}+\epsilon_{4}^{\perp} \mu \epsilon_{3}^{\perp \nu} \epsilon_{2}^{\perp} \rho \alpha_{3} \alpha_{2} \alpha_{1}\right) \epsilon_{1}^{\perp} . \epsilon_{4}^{\perp}\right. \\
& \left.+\left(\epsilon_{2}^{\perp} \mu \epsilon_{1}^{\perp \nu} \epsilon_{4}^{\perp \rho} \rho \alpha_{3} \alpha_{4} \alpha_{1}+\epsilon_{3}^{\perp} \mu \epsilon_{4}^{\perp \nu} \epsilon_{1}^{\perp \rho} \alpha_{4} \alpha_{1} \alpha_{2}\right) \epsilon_{2}^{\perp} . \epsilon_{3}^{\perp}\right),
\end{aligned}
$$

In summary, the parity odd part of the $D=6$ bare module has 9 structures which transform under $S_{3}$ as

$$
9=3 \cdot \mathbf{1}_{\mathbf{A}} \oplus 3 \cdot \mathbf{2}_{\mathbf{M}} .
$$


$\boldsymbol{D}=\mathbf{5}$. Let us discuss parity even case first. As the transverse symmetry is $\mathrm{SO}(2)$ it is best to consider $\mathrm{t}, \mathrm{v}$ and $\mathrm{s}$ as states with charge $\pm 2, \pm 1$ and 0 respectively. In the asymptotic dimension, in the $t^{\otimes 4}$ subsector, there are six parity even $\mathbb{Z}_{2} \times \mathbb{Z}_{2}$ symmetric singlets as denoted in the first two lines of figure 2 ; these transformed in the $2 \cdot \mathbf{3}=$ $2 \cdot \mathbf{2}_{\mathbf{M}}+2 \cdot \mathbf{1}_{\mathbf{S}}$. In the previous subsection the relationship (D.12) removed one of these $\mathbf{1}_{\mathrm{S}}$ representations leaving us with $2 \cdot \mathbf{2}_{\mathrm{M}}+\mathbf{1}_{\mathbf{S}}$. The number of states in the $\mathrm{t}^{\otimes 4}$ sector is further reduced as we now see. In $D=5$ all states in this sector have charges \pm 2 states. The only neutral combination has charge assignments $2,2,-2,-2$. This set of charges has a 3 dimensional orbit which transform in $\mathbf{3}$ of $S_{3} \cdot{ }^{125}$

In the same way the neutral charge assignments the sector $\mathrm{t}^{\otimes 2} \mathrm{v}^{\otimes 2}$ is $2,-2,1,-1$. We have 6 neutral combinations of these charges that transform in the $\boldsymbol{6}_{\text {left }}$ under $S_{3}$. The number 6 is three less than the 9 parity invariant structures in the $\mathrm{t}^{\otimes 2} \mathrm{v}^{\otimes 2}$ sector in $D \geq 6$ (see subfigs 3) and 4) ) in figure 2). ${ }^{126}$

Next, the subsector $\mathrm{t}^{\otimes 3} s$ clearly doesn't contain any singlets in $D=5$ (recall that this sector had one singlet for $D \geq 6$, see sub fig 5 ) of figure 2 .

It is easy to do a similar counting in the sectors $\mathrm{t}^{\otimes 2} s^{\otimes 2}, \mathrm{v}^{\otimes 2} s^{\otimes 2}, \mathrm{v}^{\otimes 4}$ and $s^{\otimes 4}$. In these sectors we find $3,3,3$ and 1 states respectively. All these results are the same as for $D \geq 6$; the corresponding structures are all parity even and all transform in the same representations of $S_{3}$ (and in fact are given by the same expressions) as for $D \geq 6$.

The chief new result is in the sector $\mathrm{tv}^{\otimes 2} s$. In this sector we have two possible charge assignments; the $(2,-1,-1,0)$ and the $(-2,1,1,0)$. One of these charge assignments generate a $\mathbf{3}_{\mathbf{S}}$ and the other $\mathbf{3}_{\mathbf{A}}$. giving us a total of six states, twice the number in $D \geq 6$. 3 of these states are parity even, transform in the $\mathbf{3}$ and are depicted in subfig 7 ) of figure 2. The other $\mathbf{3}_{\mathbf{A}}$ is parity odd and is given by the explicit expression

$$
\begin{aligned}
& \widetilde{\varepsilon}_{\mu \nu}^{2}\left(\left(\epsilon_{4}^{\perp} \mu \epsilon_{2}^{\perp \nu} \alpha_{2} \alpha_{1} \alpha_{3}^{2}+\epsilon_{1}^{\perp} \mu \epsilon_{3}^{\perp \nu} \alpha_{3} \alpha_{4} \alpha_{2}^{2}\right) \epsilon_{4}^{\perp} . \epsilon_{1}^{\perp}\right. \\
& \left.+\left(\epsilon_{2}^{\perp}{ }^{\mu} \epsilon_{4}^{\perp \nu} \alpha_{4} \alpha_{3} \alpha_{1}^{2}+\epsilon_{3}^{\perp} \epsilon_{1}^{\perp \nu} \alpha_{1} \alpha_{2} \alpha_{4}^{2}\right) \epsilon_{2}^{\perp} . \epsilon_{3}^{\perp}\right), \\
& -\left(\widetilde { \varepsilon } _ { \mu \nu } ^ { 2 } \left(\left(\epsilon_{4}^{\perp} \mu \epsilon_{3}^{\perp}{ }^{\nu} \alpha_{3} \alpha_{1} \alpha_{2}^{2}+\epsilon_{1}^{\perp} \epsilon_{2}^{\perp}{ }^{\nu} \alpha_{2} \alpha_{4} \alpha_{3}^{2}\right) \epsilon_{4}^{\perp} . \epsilon_{1}^{\perp}\right.\right. \\
& \left.\left.+\left(\epsilon_{3}^{\perp \mu} \epsilon_{4}^{\perp \nu} \alpha_{4} \alpha_{2} \alpha_{1}^{2}+\epsilon_{2}^{\perp \mu} \epsilon_{1}^{\perp \nu} \alpha_{1} \alpha_{3} \alpha_{4}^{2}\right) \epsilon_{3}^{\perp} . \epsilon_{2}^{\perp}\right)\right), \\
& -\left(\widetilde { \varepsilon } _ { \mu \nu } ^ { 2 } \left(\left(\epsilon_{2}^{\perp} \mu \epsilon_{4}^{\perp \nu} \alpha_{4} \alpha_{1} \alpha_{3}^{2}+\epsilon_{1}^{\perp} \mu \epsilon_{3}^{\perp} \nu \alpha_{3} \alpha_{2} \alpha_{4}^{2}\right) \epsilon_{2}^{\perp} . \epsilon_{1}^{\perp}\right.\right. \\
& \left.\left.+\left(\epsilon_{4}^{\perp \mu} \epsilon_{2}^{\perp \nu} \alpha_{2} \alpha_{3} \alpha_{1}^{2}+\epsilon_{3}^{\perp} \mu \epsilon_{1}^{\perp \nu} \alpha_{1} \alpha_{4} \alpha_{2}^{2}\right) \epsilon_{4}^{\perp} . \epsilon_{3}^{\perp}\right)\right) .
\end{aligned}
$$

In summary, in $D=5$ we have 22 parity even structures which transform in

$$
22=7 \cdot \mathbf{1}_{\mathbf{S}}+7 \cdot \mathbf{2}_{\mathbf{M}}+1 \cdot \mathbf{1}_{\mathbf{A}}
$$

\footnotetext{
${ }^{125}$ The tensor structures in question can be taken to be either that represented in subfig 1) or subfig 2) of figure 2 (these two tensor structures, which are distinct in $D \geq 7$ are proportional to each other in $D=5$; this is what leads to the reduction of 6 to 3 structures).

${ }^{126}$ In this case the independent tensor structures can be taken to be that depicted in subfig 4 ) of figure 2 (the tensor structure of subfig 3 ) is linearly related to that of subfig 4) in $D=5$ - this accounts for the reduction of structures from 9 to 6 .
} 
On the other hand we have 3 parity odd structures which transform in the

$$
3=\mathbf{1}_{\mathrm{A}}+\mathbf{2}_{\mathrm{M}}
$$

$\boldsymbol{D}=4$. As in the case of photons, the graviton polarizations are essentially numbers. Hence the result for the counting of structures is the same as the one in the case of photons. Physically this is to be expected since the massless particles in $D=4$ have two helicities regardless of their spin. More concretely, due the fact that $\epsilon_{i}^{\perp} \mathrm{s}$ are basically numbers, any appearance of the quantity $\epsilon_{i}^{\perp 2}$ in the bare module can be replaced by $\alpha_{i}^{2}$ due to the tracelessness of the graviton fluctuations (see eqn (2.15)). Recall that in order to enumerate the possible $\mathrm{SO}(D-3)$ singlets in subsection 3.1, we had decomposed the effective polarization as $\rho=(\mathrm{s} \oplus \mathrm{v} \oplus \mathrm{t})$ (see (3.1)). In $D=4$, the $\mathrm{t}$ part can be replaced by $\mathrm{s}$ using (2.15). Hence at the level of group theoretic enumeration undertaken in subsection 3.1, the counting for gravitons is the same as that of photons. In terms of explicit bare module structures, consider the following two possible graviton bare module structures in $D=4$

$$
\left.\left(\epsilon_{1}^{\perp} \cdot \epsilon_{2}^{\perp}\right) \alpha_{1} \alpha_{2} \alpha_{3}^{2} \alpha_{4}^{2}\right|_{\mathbb{Z}_{2} \otimes \mathbb{Z}_{2}},\left.\quad\left(\epsilon_{1}^{\perp} \cdot \epsilon_{2}^{\perp}\right)\left(\epsilon_{2}^{\perp} \cdot \epsilon_{3}^{\perp}\right) \alpha_{1} \alpha_{3} \alpha_{4}^{2}\right|_{\mathbb{Z}_{2} \otimes \mathbb{Z}_{2}}
$$

Using (2.15), we can replace $\epsilon_{2}^{\perp} 2$ appearing in the second structure by $\alpha_{2}^{2}$ and hence is equal to one of the structures present in the $S_{3}$ orbit of the first structure. The bare module for the gravitons in $D=4$ therefore is given by that of the photons after the replacement $\epsilon_{i}^{\perp} \rightarrow \alpha_{i} \epsilon_{i}^{\perp}$ and $\alpha_{i} \rightarrow \alpha_{i}^{2}$. In equations the parity even module is given by,

$$
\begin{aligned}
g_{\mathbf{3} \rightarrow \mathbf{S}}^{D=4} & =\left(\epsilon_{1}^{\perp} \epsilon_{2}^{\perp} \mu\right)\left(\epsilon_{3}^{\perp} \nu \epsilon_{4}^{\perp} \nu\right) \alpha_{1} \alpha_{2} \alpha_{3} \alpha_{4}, \\
g_{\mathbf{3}, 2}^{(1)} & =\left(\epsilon_{1}^{\perp} \epsilon_{2}^{\perp} \nu \alpha_{1} \alpha_{2} \alpha_{3}^{2} \alpha_{4}^{2}+\epsilon_{3}^{\perp} \epsilon_{4}^{\perp}{ }^{\mu} \alpha_{3} \alpha_{4} \alpha_{1}^{2} \alpha_{2}^{2}\right), \\
g_{\mathbf{3}, 2}^{(2)} & =\left(\epsilon_{1}^{\perp} \epsilon_{3}^{\perp \nu} \alpha_{1} \alpha_{3} \alpha_{2}^{2} \alpha_{4}^{2}+\epsilon_{2}^{\perp} \epsilon_{4}^{\perp}{ }^{\mu} \alpha_{2} \alpha_{4} \alpha_{1}^{2} \alpha_{3}^{2}\right) \\
g_{\mathbf{3}, 2}^{(3)} & =\left(\epsilon_{1}^{\perp} \epsilon_{4}^{\perp}{ }^{\perp} \alpha_{1} \alpha_{4} \alpha_{3}^{2} \alpha_{2}^{2}+\epsilon_{3}^{\perp} \epsilon_{2}^{\perp}{ }^{\mu} \alpha_{3} \alpha_{2} \alpha_{1}^{2} \alpha_{4}^{2}\right) \\
g_{\mathbf{S}, 1} & =\left(\alpha_{1}^{2} \alpha_{2}^{2} \alpha_{3}^{2} \alpha_{4}^{2}\right)
\end{aligned}
$$

Similarly the parity odd module is generated by,

$$
h_{\mathbf{S}, 1}^{D=4}=\left.\left(N(\widetilde{\varepsilon})_{\mu} \epsilon_{4 \mu}^{\perp}\right) \alpha_{1}^{2} \alpha_{2}^{2} \alpha_{3}^{2} \alpha_{4}\right|_{\mathbb{Z} 2 \times \mathbb{Z} 2} \quad \text { and } \quad h_{\mathbf{S}, 2}^{D=4}=\left(N(\widetilde{\varepsilon})_{\mu} \epsilon_{4 \mu}^{\perp}\right) \epsilon_{1 \nu}^{\perp} \epsilon_{2 \nu}^{\perp} \alpha_{1} \alpha_{2} \alpha_{4} \alpha_{3}^{2} \|_{\mathbb{Z} 2 \times \mathbb{Z} 2} .
$$

\section{E Details concerning photon Lagrangians}

\section{E.1 Triviality of 3-F structures}

In this appendix we prove that any $3-\mathrm{F}$ structure and its descendants identically vanish when we impose equations of motion, and Bianchi identity. we write them here for convenience

$$
\partial_{\mu} F_{\mu \nu}=0, \text { and } \partial_{a} F_{b c}+\partial_{b} F_{c a}+\partial_{c} F_{a b}=0
$$

Both together imply the following, as shown

$$
\begin{aligned}
& \partial_{\mu} \partial_{\mu} F_{a b}=0 \\
\text { Using Bianchi Identity: L.H.S. } & =\partial_{\mu}\left(-\partial_{a} F_{b \mu}-\partial_{b} F_{\mu a}\right) \\
\text { Using E.o.M.: } & =0
\end{aligned}
$$


Now, let's consider a general descendant term, where some derivatives are contracted among themselves. If they act on the same $F$, (E.2) shows that any such term is just 0 . In case they act on different $F \mathrm{~s}$, we use momentum conservation to write it as

$$
k_{\mu}^{i} k_{\mu}^{j}=\frac{1}{2}\left(\left(k^{s}\right)^{2}-\left(k^{i}\right)^{2}-\left(k^{j}\right)^{2}\right)
$$

where $s$ in the superscript is for the particle label apart from $i, j$. Each of the three terms on r.h.s. are 0 by (E.2). Therefore, all the descendants of any 3-F structure are 0 . Next we consider the contraction of derivatives with $F$ s.

\section{E.1.1 6 derivatives}

There is only one term that one can write down. Firstly, both the indices of an $F$ can not contract with the same particle momenta, because of anti-symmetry of $F$. Therefore, one index of $F^{1}$ contracts with $k^{2}$ and other with $k^{3} \cdot{ }^{127}$ Similarly for $F^{2}$ and $F^{3}$. The term is

$$
k_{c}^{1} k_{e}^{1} F_{a b}^{1} k_{a}^{2} k_{f}^{2} F_{c d}^{2} k_{b}^{3} k_{d}^{3} F_{e f}^{3}
$$

Under $1 \leftrightarrow 2$ this is antisymmetric, therefore 0 .

\section{E.1.2 4 derivatives}

Next consider a basic structure with 4 derivatives. As there are a total of 4 derivatives, both free indices of at least one field strength must contract with derivatives. Actually both free indices of exactly one field strength must contract with derivatives. ${ }^{128}$ Let this special field strength be $F^{3}$. By use of momentum conservation we can choose the momenta that contract the indices of $F^{3}$ to be $k^{1}$ and $k^{2}$, again no contraction with the same $k^{i}$. One momentum index must also contract with one of the indices of $F^{1}$ - we can use momentum conservation to set this momentum to either $k_{1}$ or $k_{3}$. Similarly we can choose to ensure that the momentum that contracts $F^{2}$ is either $k_{2}$ or $k_{3}$. This gives us a total of 4 basic structures

$$
\begin{aligned}
& F_{\mu \nu}^{3}\left(k_{\mu}^{1} k_{\nu}^{2} k_{a}^{1} k_{b}^{2} F_{a \beta}^{1} F_{b \beta}^{2}\right) \\
& F_{\mu \nu}^{3}\left(k_{\mu}^{1} k_{\nu}^{2} k_{a}^{3} k_{b}^{3} F_{a \beta}^{1} F_{b \beta}^{2}\right) \\
& F_{\mu \nu}^{3}\left(k_{\mu}^{1} k_{\nu}^{2}\left(k_{a}^{1} k_{b}^{3}+k_{a}^{3} k_{b}^{2}\right) F_{a \beta}^{1} F_{b \beta}^{2}\right) \\
& F_{\mu \nu}^{3}\left(k_{\mu}^{1} k_{\nu}^{2}\left(k_{a}^{1} k_{b}^{3}-k_{a}^{3} k_{b}^{2}\right) F_{a \beta}^{1} F_{b \beta}^{2}\right)
\end{aligned}
$$

The expressions in the first three lines of (E.5) involve the contraction of $F_{\mu \nu}^{3}$ with structures that are symmetric under $1 \leftrightarrow 2$. These structures all vanish after integrating over $k_{1}$ and $k_{2}$. On the other hand the structure in the last line of (E.5) vanishes because of equations of motion $\left(k_{a}^{1} F_{a \beta}^{1}=0\right.$ and $\left.k_{b}^{2} F_{b \beta}^{2}=0\right)$.

\footnotetext{
${ }^{127}$ The possibility that both indices of $F^{1}$ contract with $F^{2}$ or $F^{3}$ is equivalent to this up to momentum conservation.

${ }^{128}$ If the four were to contract with the 4 indices of 2 field strengths then the indices of the remaining field strength would have to contract with each other, and that is not allowed.
} 


\section{E.1.3 2 derivatives}

First, is the case when both the derivatives are contracted with the same $F$, say $F^{3}$. There is one possible term, up to momentum conservation

$$
k_{\mu}^{1} k_{\nu}^{2} F_{a b}^{1} F_{b a}^{2} F_{\mu \nu}^{3}
$$

Again, indices of $F$ can not contract with the same particle momenta. This term is antisymmetric under $1 \leftrightarrow 2$, and therefore 0 .

Next, consider the case when both the derivatives are contracted with different $F$ s, say $F^{1}$ and $F^{2}$. Similar to the argument for 4 derivative case, there are 4 terms

$$
\begin{aligned}
& F_{\mu \nu}^{3}\left(k_{a}^{1} k_{b}^{2} F_{a \mu}^{1} F_{b \nu}^{2}\right) \\
& F_{\mu \nu}^{3}\left(k_{a}^{3} k_{b}^{3} F_{a \mu}^{1} F_{b \nu}^{2}\right) \\
& F_{\mu \nu}^{3}\left(\left(k_{a}^{1} k_{b}^{3}+k_{a}^{3} k_{b}^{2}\right) F_{a \mu}^{1} F_{b \nu}^{2}\right) \\
& F_{\mu \nu}^{3}\left(\left(k_{a}^{1} k_{b}^{3}-k_{a}^{3} k_{b}^{2}\right) F_{a \mu}^{1} F_{b \nu}^{2}\right)
\end{aligned}
$$

As for the case of 4 derivatives, the first three of these terms are 0 because of anti-symmetry under $1 \leftrightarrow 2$ and the last one is 0 because of equations of motion.

\section{E.2 Local photon Lagrangians and polynomial S-matrices}

In order to make contact with the S-matrices described earlier in this paper we now specialize to Lorentz gauge $\partial . A=0$. In this case the Maxwell equation reduces to ${ }^{129}$

$$
\partial^{2} A_{\mu}=0, \quad \partial . A=0 .
$$

In this gauge it follows that different $L_{4}$ belong to the same equivalence class if they differ only by total derivatives once one imposes (E.8) In momentum space

$$
\begin{aligned}
A_{\mu}(x) & =\int \frac{d^{d} k}{(2 \pi)^{d}} e^{i k . x} \epsilon_{\mu}(k) \\
L_{4} & =\int \prod_{i} \frac{d^{d} k}{(2 \pi)^{d}} e^{i\left(\sum_{j} k_{j} x_{j}\right)} \widetilde{L}_{4}^{\mu_{1} \mu_{2} \mu_{3} \mu_{4}}\left(k_{1}, k_{2}, k_{3}, k_{4}\right) \epsilon_{\mu_{1}}\left(k_{1}\right) \epsilon_{\mu_{2}}\left(k_{2}\right) \epsilon_{\mu_{3}}\left(k_{3}\right) \epsilon_{\mu_{4}}\left(k_{4}\right) \\
k_{i} . \epsilon_{i} & =0, \quad\left(k_{1}\right)_{\mu} \widetilde{L}_{4}^{\mu_{1} \mu_{2} \mu_{3} \mu_{4}}\left(k_{1}, k_{2}, k_{3}, k_{4}\right)=0 \quad \text { similar eq for } 1 \leftrightarrow \mathrm{i}
\end{aligned}
$$

The equations of motion (E.8) and momentum conservation merely impose (4.7). As $\widetilde{L}_{4}^{\mu_{1} \mu_{2} \mu_{3} \mu_{4}}\left(k_{1}, k_{2}, k_{3}, k_{4}\right)$, subject to (4.7), is the gauge invariant tree level S-matrix obtained from our theory, it follows immediately that equivalence classes of $L_{4}$ are labelled by their S-matrices as in the scalar case studied in the previous subsubsection. We believe ${ }^{130}$ that

\footnotetext{
${ }^{129}$ While $A_{\mu}$ is harmonic only in Lorentz gauge, it follows from the Bianchi identity and the Maxwell equations that $\partial^{2} F_{\mu \nu}=0$ in any gauge. Consequently if we choose to work in a gauge invariant manner, Lagrangians that differ from each other by terms that include a factor of $\partial^{2} F_{\mu \nu}=0$ are also in the same equivalence class.

${ }^{130}$ Eq. (E.9) can be re expressed entirely in terms of Field strengths using (2.10), (2.11), (2.16) and (2.17). It should be possible to demonstrate that resulting expression is a local functional of Field strengths (at least in parity invariant situations). We have not undertaken this exercise; we leave its completion to the interested reader.
} 
every local gauge invariant S-matrix defines an equivalence class of local and manifestly gauge invariant quartic Lagrangians $L_{4}$. It follows that local 4-photon S-matrices are in one to one correspondence with equivalence classes $L_{4}$ in (4.10). In subsection 4.2 later in this section we turn to the problem of enumerating inequivalent quartic Lagrangians $L_{4}$, and so, effectively, inequivalent 4 photon S-matrices.

\section{F S-matrices from Lagrangians no more than cubic in $R_{\alpha \beta \gamma \delta}$}

\section{F.1 Identities of the Riemann tensor}

In this section we list all the identities and symmetry properties of the Riemann tensor that we will be using in the subsequent analysis to determine the diffeomorphism invariant Lagrangian structures.

1. The symmetry properties of the Riemann tensor,

$$
R_{a b c d}=-R_{b a c d}=-R_{a b d c}, \quad R_{a b c d}=R_{c d a b}
$$

2. The algebraic Bianchi identity

$$
R_{a b c d}+R_{a c d b}+R_{a d b c}=0
$$

3. The differential Bianchi identity

$$
\nabla_{a} R_{b c d e}+\nabla_{b} R_{c a d e}+\nabla_{c} R_{a b d e}=0
$$

4. The contracted Bianchi identity, which we get from the differential Bianchi identity by contracting the Riemann tensor appropriately.

$$
\nabla_{a} R_{c e}+\nabla^{b} R_{c a b e}-\nabla_{c} R_{a e}=0
$$

5. Commutator of derivatives

$$
\left[\nabla_{f}, \nabla_{e}\right] R_{a b c d}=R_{p b c d} R_{a f e}^{p}+R_{a p c d} R_{b f e}^{p}+R_{a b p d} R_{c f e}^{p}+R_{a b c p} R_{d f e}^{p}
$$

We will be using these identities and symmetry properties of the Riemann tensor along with integration by parts judiciously to fix the independent Lagrangian terms.

Through this appendix we focus only on results that are true in every dimension; in particular we ignore dimension dependent structures like $\epsilon$ and never make use of special identities for the Riemann tensor that work only in special dimensions. 


\section{F.2 Terms quadratic in $R_{\alpha \beta \gamma \delta}$}

The unique Lagrangian that is linear in $R_{\alpha \beta \gamma \delta}$ is clearly the familiar two derivative Einstein Lagrangian itself.

Let us now turn to terms built out of two copies of the Riemann tensor. One Lagrangian of this form is the Gauss-Bonnet Lagrangian (4.16) which we reproduce here for convenience:

$$
\begin{aligned}
S_{G B} & =\int \sqrt{-g} \delta_{[a}^{g} \delta_{b}^{h} \delta_{c}^{i} \delta_{d]}^{j} R_{a b}{ }^{g h} R_{c d}{ }^{i j} \\
& \propto \int \sqrt{-g}\left(R^{2}-4 R^{\mu \nu} R_{\mu \nu}+R^{\mu \nu \rho \sigma} R_{\mu \nu \rho \sigma}\right) .
\end{aligned}
$$

Since we are classifying terms up to 'equations of motion' this Lagrangian is equivalent to

$$
S=\int \sqrt{-g} R_{a b c d} R^{a b c d}
$$

We will now argue that every other Lagrangian term quadratic in two Riemann tensors is equivalent to (F.7), plus terms that are cubic or higher order in $R_{a b c d}$.

To see how this works, we first note that the symmetries of the Riemann tensor and the algebraic Bianchi identity ensure that the second potentially independent index contraction of 4 derivative two Riemann terms

$$
S=\int \sqrt{-g} R_{a b c d} R^{a c b d}
$$

is in fact proportional to (F.7). Using (F.1), we can systematically relate (F.8) to (F.7) as follows

$$
\begin{aligned}
R_{a b c d} R^{a c b d} & =R_{a b c d}\left(-R^{a b d c}-R^{a d c b}\right) \\
& =R_{a b c d} R^{a b c d}-R_{a b c d} R^{a c b d} \\
\therefore R_{a b c d} R^{a c b d} & =\frac{1}{2} R_{a b c d} R^{a b c d}
\end{aligned}
$$

where in the first line we have used the algebraic Bianchi identity and in the second line we have used the symmetry properties of the Riemann tensor.

We next turn two Riemann terms involving derivatives. In all such terms the derivative indices are either contracted with indices in one of the Riemann tensor, or with some other derivative. In the first case an integration by parts can always be used to have the derivative whose index contracts with (say) the $a$ index of $R_{a b c d}$, act on (derivatives of) $R_{a b c d}$ itself. We can then move $\nabla_{a}$ through all the other derivatives acting on $R_{a b c d}$ - along the way generating three Riemann terms (the extra factor of Riemann comes from commuting derivatives) which we are allowed at this stage to ignore - until we obtain an expression that is some derivative of $\nabla^{a} R_{a b c d}$. The contracted Bianchi identity (F.3) then sets this term equal to expressions involving $R_{\mu \nu}$ which are field redefinition trivial. We present two examples of such manipulations 


$$
\begin{aligned}
S & =\int \nabla_{a} R_{b c f e} \nabla^{b} R^{a c f e} \\
& =-\int\left(\nabla^{b} \nabla_{a} R_{b c f e}\right) R^{a c f e}+C_{\partial \mathcal{M}} \\
& =\widehat{C}_{R_{\mu \nu}}+C_{\partial \mathcal{M}}+\widetilde{C}_{R^{3}}
\end{aligned}
$$

$$
\begin{aligned}
S & =\int \sqrt{g} \nabla_{a} \nabla_{b} R_{e c f d} \nabla^{e} \nabla^{f} R^{a c b d} \\
& =\int \sqrt{g} \nabla^{e} \nabla^{f} \nabla_{a} \nabla_{b} R_{e c f d} R^{a c b d}+C_{\partial \mathcal{M}} \\
& =\widehat{C}_{R_{\mu \nu}}+C_{\partial \mathcal{M}}+\widetilde{C}_{\nabla R \nabla R R}
\end{aligned}
$$

(here $\widehat{C}_{R_{\mu \nu}}$ denotes a Lagrangian term which has at least one factor of $R_{\mu \nu}$ or its derivatives. $C_{\partial \mathcal{M}}$ denotes a total derivative and $\widetilde{C}_{R^{3}}$ denotes a term that is cubic or higher order in Riemann tensors).

Next, terms in which derivative indices are contracted with one another can be converted into the first kind of terms (those in which derivative indices are contracted with an a free index in the Riemann tensor) by use of the differential Bianchi identity. For example

$$
\begin{aligned}
S & =\int \sqrt{g} \nabla_{\mu} R_{a b c d} \nabla^{\mu} R^{a c b d} \\
& =\int \sqrt{g}\left(-\nabla_{a} R_{b \mu c d}-\nabla_{b} R_{\mu a c d}\right) \nabla^{\mu} R^{a c b d} \\
& =\int \sqrt{g} \nabla^{\mu}\left(-\nabla_{a} R_{b \mu c d}-\nabla_{b} R_{\mu a c d}\right) R^{a c b d}+C_{\partial \mathcal{M}} \\
& =\int \sqrt{g}\left(-\nabla_{a} \nabla^{\mu} R_{b \mu c d}-\nabla_{b} \nabla^{\mu} R_{\mu a c d}\right) R^{a c b d}+C_{\partial \mathcal{M}}+\widetilde{C}_{R^{3}} \\
& =\widehat{C}_{R_{\mu \nu}}+C_{\partial \mathcal{M}}+\widetilde{C}_{R^{3}}
\end{aligned}
$$

where in the second line we have used the differential Bianchi identity. In the third line we have used integration by parts to get the total derivative term $C_{\partial \mathcal{M}}$. In the fourth line we have used commutator of derivatives to get the $\widetilde{C}_{R^{3}}$ term which is of higher order in Riemann tensor and hence will be dealt with when we classify terms with three Riemann tensors. Finally we use the contracted Bianchi identity to relate this to the Ricci tensor term $\widehat{C}_{R_{\mu \nu}}$.

For another example of such terms consider

$$
\begin{aligned}
S & =\int \sqrt{g} \nabla_{\mu} \nabla_{\nu} R_{a b c d} \nabla^{\mu} \nabla^{\nu} R^{a c b d} \\
& =\int \sqrt{g} \nabla_{\nu} \nabla^{\mu} \nabla_{\mu} R_{a b c d} R^{a c b d}+C_{\partial \mathcal{M}} \\
& =\int \sqrt{g} \nabla_{\nu} \nabla^{\mu} \nabla_{\mu}\left(-\nabla_{a} R_{b \nu c d}-\nabla_{b} R_{\nu a c d}\right) R^{a c b d}+C_{\partial \mathcal{M}} \\
& =\widehat{C}_{R_{\mu \nu}}+C_{\partial \mathcal{M}}+\widetilde{C}_{\nabla R \nabla R R}
\end{aligned}
$$


Similar manipulations can be used to show that all expressions involving derivatives of two Riemann tensors can be turned into terms involving three or more Riemann tensor, up to terms that can be removed by field redefinitions.

\section{F.3 Terms cubic in the Riemann tensor}

Finally let us consider terms built out of three copies of the Riemann tensor. Let us first study terms with no derivatives. It is easy to convince oneself that there are two independent terms of this form ([40]).

$$
R^{p q r s} R_{p q}^{t u} R_{r s t u}, \quad R^{p q r s} R_{p r}^{t}{ }^{u} R_{q t s u}
$$

All possible non-zero index contractions of three Riemann tensors to yield scalars can be obtained from this two by repeated application of the symmetry properties and the algebraic Bianchi identities. Let us consider an explicit example: $R_{p q}{ }^{r s} R_{r t s u} R^{t u}{ }_{p q}$

$$
\begin{aligned}
R_{p q}{ }^{r s} R_{r t s u} R_{p q}^{t u} & =R_{p q}{ }^{r s}\left(-R_{r s u t}-R_{r u t s}\right) R_{p q}^{t u} \\
& =R_{p q}{ }^{r s} R_{r s t u} R_{p q}^{t u}-R_{p q}{ }^{r s} R_{r t s u} R_{p q}^{t u} \\
\therefore R_{p q}{ }^{r s} R_{r t s u} R_{p q}^{t u} & =\frac{1}{2} R_{p q}^{r s} R_{r s t u} R_{p q}^{t u}
\end{aligned}
$$

In the second line we have used the algebraic Bianchi identity and subsequently used the symmetry properties of the Riemann tensor. Similar manipulations can be performed for any non-zero contraction of three Riemann tensors (and no additional derivatives) to reduce it to one of the two forms or a linear combination of both.

We note that the combination

$$
R^{p q r s} R_{p q}^{t u} R_{r s t u}-2 R^{p q r s} R_{p r}^{t}{ }^{u} R_{q t s u},
$$

which up to terms proportional to $R_{\mu \nu}$ is proportional to the second Lovelock term

$$
\begin{aligned}
\chi_{6}= & \frac{1}{8} \epsilon_{a b c d e f} \epsilon^{g h i j k l} R_{a b}{ }^{g h} R_{c d}{ }^{i j} R_{e f}{ }^{k l} \\
= & 4 R_{a b}{ }^{c d} R_{c d}{ }^{e f} R_{e f}{ }^{a b}-8 R_{a b}{ }^{c}{ }^{d} R_{c d}{ }^{e f} R_{e f}{ }^{a b}-24 R_{a b c d} R^{a b c} e^{d e}+3 R_{a b c d} R^{a b c d} R \\
& +24 R_{a b c d} R^{a c} R^{b d}+16 R_{a}^{b} R_{b}{ }^{c} R_{c}{ }^{a}-12 R_{a}{ }^{b} R_{b}{ }^{a} R+R^{3}
\end{aligned}
$$

does not contribute to the graviton 3 point function. This may be verified using the fact that with our standard choice of polarization, the on-shell Riemann tensor evaluates to

$$
\begin{aligned}
R_{a b c d} & =\frac{1}{2} F_{a b} F_{c d} \\
F_{a b} & =\left(k_{a} e_{b}-k_{b} e_{a}\right), \quad h_{a b}=e_{a} e_{b}
\end{aligned}
$$

an expression that is both gauge invariant and satisfies the necessary symmetry properties of the Riemann tensor (also - on-shell — both the algebraic Bianchi identity and the differential Bianchi identity are satisfied). 
We now turn to a discussion of terms with derivatives on three copies of the Riemann tensor. The basis for structures with two derivatives on three Riemann tensors have been discussed in [40].

$$
\begin{array}{ll}
R^{\text {pqrs }} R_{p}^{\text {tuv }} \nabla_{v} \nabla_{s} R_{\text {qtru }}, & R^{\text {pqrs }} \nabla_{q} R^{\text {tuv }}{ }_{p} \nabla_{s} R_{\text {tuvr }} \\
R^{\text {pqrs }} \nabla_{r} R^{\text {tuv }}{ }_{p} \nabla_{s} R_{\text {tuvq }}, & R^{\text {pqrs }} \nabla^{v} R_{p r}^{\text {tu }} \nabla_{v} R_{\text {tqus }}
\end{array}
$$

We will now demonstrate that all such terms are field redefinition equivalent to terms involving four or more Riemann tensors.

1

$$
\begin{aligned}
\int \sqrt{g} R^{p q r s} R_{p}^{t u v} \nabla_{v} \nabla_{s} R_{q t r u} & =\int \sqrt{g} R^{p q r s} R_{p}^{t u v} \nabla_{v}\left(-\nabla_{r} R_{q t u s}-\nabla_{u} R_{q t s r}\right) \\
& =-\int \sqrt{g} R^{p q s r} R_{p}^{t u v} \nabla_{v} \nabla_{r} R_{q t s u}+C_{R^{4}}^{\prime} \\
\therefore \int \sqrt{g} R^{p q r s} R_{p}^{t u v} \nabla_{v} \nabla_{s} R_{q t r u} & \sim \frac{1}{2} C_{R^{4}}^{\prime}
\end{aligned}
$$

2

$$
\begin{aligned}
\int \sqrt{g} R^{p q r s} \nabla_{r} R_{p}^{t u v} \nabla_{s} R_{t u v q} & =-\int \sqrt{g} R^{p q r s} \nabla_{s} \nabla_{r} R_{p}^{\text {tuv }} R_{t u v q}+C_{\partial \mathcal{M}} \\
& \sim C_{R^{4}}^{\prime}+C_{\partial \mathcal{M}}
\end{aligned}
$$

3

$$
\begin{aligned}
& \int \sqrt{g} R^{p q r s} \nabla^{v} R_{p r}^{t u} \nabla_{v} R_{t q u s}=-\int \sqrt{g}\left(\nabla^{v} R^{p q r s} R_{p r}^{t u} \nabla_{v} R_{t q u s}\right. \\
& \left.+R^{p q r s} R_{p r}^{t}{ }_{p r} \nabla^{v} \nabla_{v} R_{t q u s}\right)+C_{\partial \mathcal{M}} \\
& \int \sqrt{g} R^{p q r s} \nabla^{v} R_{p r}^{t u} \nabla_{v} R_{t q u s}=\frac{-1}{2} \int \sqrt{g} R^{p q r s} R_{p r}^{t u} \nabla^{v} \nabla_{v} R_{t q u s}+C_{\partial \mathcal{M}} \\
& \sim \widehat{C}_{R_{\mu \nu}}+C_{R^{4}}^{\prime}+C_{\partial \mathcal{M}}
\end{aligned}
$$

4

$$
\begin{aligned}
& \int \sqrt{g} R^{p q r s} \nabla_{q} R_{t u v p} \nabla_{s} R_{r}^{t u v}=-\int \sqrt{g} R^{p q r s}\left(\nabla_{v} R_{t u p q}+\nabla_{p} R_{t u q v}\right) \nabla_{s} R_{r}^{t u v} \\
& \int \sqrt{g} R^{p q r s} \nabla_{q} R_{t u v p} \nabla_{s} R_{r}^{\text {tuv }}=\frac{-1}{2} \int \sqrt{g} R^{p q r s} \nabla_{v} R_{t u p q} \nabla_{s} R_{r}^{t u v} \\
& =\frac{-1}{2} \int \sqrt{g} R^{p q r s} \nabla_{v} R_{t u p q}\left(-\nabla^{v} R_{r s}^{t u}-\nabla_{r} R_{s}^{t u}\right) \\
& \int \sqrt{g} R^{p q r s} \nabla_{q} R_{t u v p} \nabla_{s} R_{r}^{t u v}=\frac{1}{4} \int \sqrt{g} R^{p q r s} \nabla_{v} R_{t u p q} \nabla^{v} R_{r s}^{t u} \\
& \sim \widehat{C}_{R_{\mu \nu}}+C_{R^{4}}^{\prime}
\end{aligned}
$$


Although we have only explicitly demonstrated the triviality of derivative terms involving two derivatives above, the method used to demonstrate this triviality is general. In order to demonstrate this, we have used the differential Bianchi identity and total derivatives judiciously to bring these structures to the schematic form (say)

$$
R^{p q r s} R_{p}{ }^{t u v} \nabla_{v} \nabla_{u} R_{q t s r}
$$

As the reader can see this is a higher point function due to the antisymmetry of the $\nabla_{v}$ and $\nabla_{u}$. This extends to higher derivative terms as well.

\section{G Single letter index for photons and gravitons}

In section 4.2 and 4.3, we have argued that the single letter indices for scalars, photons and gravitons are,

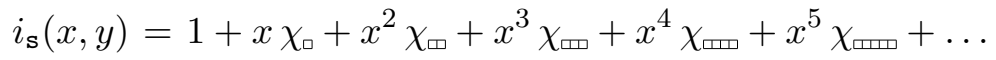

$$
\begin{aligned}
& i_{\mathrm{v}}(x, y)=x \chi_{\boxminus}+x^{2} \chi_{\boxplus}+x^{3} \chi_{\boxplus}+x^{4} \chi_{\boxminus}+x^{5} \chi_{\boxplus}+\ldots \\
& i_{\mathrm{t}}(x, y)=x^{2} \chi_{\boxplus}+x^{3} \chi_{\boxplus}+x^{4} \chi_{\boxplus}+x^{5} \chi_{\boxplus}+\ldots
\end{aligned}
$$

respectively. For scalars, it is easy to see that this series is summed to

$$
i_{\mathbf{s}}(x, y)=\left(1-x^{2}\right) \mathrm{D}(x, y)
$$

where $\mathrm{D}(x, y)$ is a function given in (4.28). In this section, we will sum the series corresponding to photons and gravitons.

$$
\begin{aligned}
& i_{\mathbf{S}}(x, y)\left(x \chi_{\square}\right)=\left(x \chi_{\square}\right)\left(1+x \chi_{\square}+x^{2} \chi_{\varpi}+x^{3} \chi_{\text {四 }}+x^{4} \chi_{\text {四 }}+\ldots\right) \\
& =x \chi_{\square}+x^{2} \chi_{\varpi}+x^{3} \chi_{\text {๓ }}+x^{4} \chi_{\text {๓ }}+\ldots \\
& x^{2}+x^{3} \chi_{\square}+x^{4} \chi_{\varpi}+x^{5} \chi_{\text {๓ }}+x^{6} \chi_{\text {巴 }}+\ldots \\
& x^{2} \chi_{\boxminus}+x^{3} \chi_{\boxplus}+x^{4} \chi_{\boxminus}+x^{5} \chi_{\boxminus 巴}+x^{6} \chi_{\boxminus 巴}+\ldots
\end{aligned}
$$

The second equality is organized such that first line is the traceless symmetrized product, second line is the trace and the third line is the antisymmetric product. Comparing to (G.1),

$$
\begin{aligned}
i_{\mathbf{s}}(x, y)\left(x \chi_{\square}\right) & =\left(i_{\mathbf{s}}(x, y)-1\right)+x^{2}\left(i_{\mathbf{s}}(x, y)\right)+x i_{\mathrm{v}}(x, y), \\
\Rightarrow x i_{\mathrm{v}}(x, y) & =\left(\left(x-x^{3}\right) \chi_{\square}-\left(1-x^{4}\right)\right) \mathrm{D}(x, y)+1 .
\end{aligned}
$$

We proceed in the same way to sum the graviton single letter index.

$$
\begin{aligned}
& i_{\mathbf{s}}(x, y)\left(x^{2} \chi_{\varpi}\right)=\left(x^{2} \chi_{\varpi}\right)\left(1+x \chi_{\square}+x^{2} \chi_{\varpi}+x^{3} \chi_{\text {四 }}+x^{4} \chi_{\text {四 }}+\ldots\right) \\
& =x^{2} \chi_{\varpi}+x^{3} \chi_{\mathrm{m}}+x^{4} \chi_{\mathrm{m}}+\ldots \\
& x^{3} \chi_{\square}+x^{4} \chi_{\text {๓ }}+x^{5} \chi_{\text {回 }}+x^{6} \chi_{\text {向 }}+\ldots \\
& x^{3} \chi_{\boxplus}+x^{4} \chi_{\boxplus}+x^{5} \chi_{\boxminus \boxplus}+x^{6} \chi_{\boxminus \text { 巴⿴囗十 }}+\ldots \\
& x^{4} \chi_{\boxminus}+x^{5} \chi_{\boxplus}+x^{6} \chi_{\boxplus}+x^{7} \chi_{\boxplus}+\ldots \\
& x^{4} \chi_{\boxplus}+x^{5} \chi_{\boxplus}+x^{6} \chi_{\boxplus}+x^{7} \chi_{\boxplus \boxplus}+\ldots \\
& x^{4}+x^{5} \chi_{\square}+x^{6} \chi_{\varpi}+x^{7} \chi_{\text {四 }}+\ldots
\end{aligned}
$$


In the second equality, the first line is the product that is symmetrized in both indices. The second line is where one index is contracted and other is symmetrized. Third is where one index is symmetrized and other anti-symmetrized. In the fourth line one index is contracted and other is anti-symmetrized. In the fifth line both the indices are anti-symmetrized and finally in the sixth line both indices are contracted. Using (G.1), this equality becomes,

$$
\begin{aligned}
i_{\mathbf{S}}(x, y)\left(x^{2} \chi_{\varpi}\right)= & \left(i_{\mathbf{s}}(x, y)-1-x \chi_{\square}\right)+x^{2}\left(i_{\mathbf{S}}(x, y)-1\right)+x\left(i_{\mathrm{v}}(x, y)-x \chi_{\boxminus}\right) \\
& +x^{3} i_{\mathrm{v}}(x, y)+x^{2} i_{\mathrm{t}}(x, y)+x^{4} i_{\mathbf{S}}(x, y), \\
\Rightarrow x^{2} i_{\mathrm{t}}(x, y)= & \left(\left(x^{2}-x^{4}\right)\left(1+\chi_{\varpi}\right)-\left(x-x^{5}\right) \chi_{\triangleright}\right) \mathrm{D}(x, y)+x^{2} \chi_{\boxminus}+x \chi_{\square} .
\end{aligned}
$$

\section{H Evaluating plethystic integrals}

In this section, we present the details of the Haar integral (see (4.32)) for scalars, photons and gravitons. The Haar integral that we need to evaluate is the following

$$
I_{j}^{D}(x):=\oint \prod_{i=1}^{\lfloor D / 2\rfloor} d y_{i} \Delta\left(y_{i}\right) i_{j}^{(4)}(x, y) / \mathrm{D}(x, y) .
$$

where $i_{j}^{(4)}(x, y)$ denotes the four particle partition function and $j$ denotes whether we are considering the partition function for scalars, photons or gravitons. The integral over $y_{i}$ in (4.32) is a closed anti-clockwise circular contour about $y_{i}=0$. From $(4.30), i_{j}^{(4)}(x, y)$ can be expressed in terms of the single letter partition function,

$i_{j}^{(4)}(x, y)=\frac{1}{24}\left(i_{j}^{4}(x, y)+6 i_{j}^{2}(x, y) i_{j}\left(x^{2}, y^{2}\right)+3 i_{j}^{2}\left(x^{2}, y^{2}\right)+8 i_{j}(x, y) i_{j}\left(x^{3}, y^{3}\right)+6 i_{j}\left(x^{4}, y^{4}\right)\right)$.

The quantity $\mathrm{D}(x, y)$ is given by

$$
\begin{aligned}
\mathrm{D}(x, y) & =\left(\prod_{i=1}^{D / 2}\left(1-x y_{i}\right)\left(1-x y_{i}^{-1}\right)\right)^{-1} & & \text { for D even } \\
& =\left((1-x) \prod_{i=1}^{\lfloor D / 2\rfloor}\left(1-x y_{i}\right)\left(1-x y_{i}^{-1}\right)\right)^{-1} & & \text { for D odd. }
\end{aligned}
$$

where $y_{i}$ are the charges under the cartan subgroup of $\mathrm{SO}(D) . \Delta\left(y_{i}\right)$ is the Van der Monde determinant for $\mathrm{SO}(D)$. For even dimensions $(D=2 N)$, the Haar measure is given by,

$$
\Delta_{e}\left(y_{i}\right)=\frac{2\left(\prod_{j=1}^{N}\left(\prod_{i=1}^{j-1}\left(y_{i}+\frac{1}{y_{i}}-y_{j}-\frac{1}{y_{j}}\right)\right)\right)^{2}}{(2 \pi i)^{N} 2^{N} N ! \prod_{i=1}^{N} y_{i}}
$$

For odd dimensions $(D=2 N+1)$, the Haar measure is given by,

$$
\Delta_{o}\left(y_{i}\right)=\frac{\left(\prod_{k=1}^{N}\left(1-y_{k}-\frac{1}{y_{k}}\right)\right)\left(\prod_{j=1}^{N}\left(\prod_{i=1}^{j-1}\left(y_{i}+\frac{1}{y_{i}}-y_{j}-\frac{1}{y_{j}}\right)\right)\right)^{2}}{(2 \pi i)^{N} N ! \prod_{i=1}^{N} y_{i}}
$$

and the integral over $y_{i}$ in (4.32) is a closed circular contour about $y_{i}=0$. 


\section{H.1 $\quad D \geq 10$}

We expect the result of the plethystic integral to stabilize for $D \geq D^{*}$ where $D^{*}$ is a critical integer. From numerical experiments (i.e. evaluating the plethystic integrals on Mathematica in a power series in $x$ to high orders in $x$ - see the next subsection) we have found strong evidence that $D^{*}=10$. Assuming this is the case, the plethystic integral for $D \geq 10$ can be evaluated in the large $D$ limit. In this subsection we will proceed to perform this evaluation. To be specific we work with the case $D=2 N$ (the case $D=2 N+1$ turns out to give the same answer).

Setting $y_{i}=e^{i \theta_{i}}$ and ignoring overall constants, we note that the Haar measure exponentiates as follows.

$$
\begin{aligned}
& \prod_{1 \leq i<j \leq N}\left(\cos _{\theta_{j}}-\cos _{\theta_{i}}\right)^{2} \\
& \propto \prod_{1 \leq i<j \leq N} \sin ^{2} \frac{\theta_{i}+\theta_{j}}{2} \sin ^{2} \frac{\theta_{i}-\theta_{j}}{2} \\
& \propto \prod_{1 \leq i<j \leq N}\left|e^{\frac{i\left(\theta_{i}+\theta_{j}\right)}{2}}-e^{\frac{-i\left(\theta_{i}+\theta_{j}\right)}{2}}\right|^{2}\left|e^{\frac{i\left(\theta_{i}-\theta_{j}\right)}{2}}-e^{\frac{-i\left(\theta_{i}-\theta_{j}\right)}{2}}\right|^{2} \\
& \propto\left(\prod_{1 \leq i<j \leq N}\left|1-e^{-i\left(\theta_{i}+\theta_{j}\right)}\right|\left|1-e^{i\left(\theta_{i}+\theta_{j}\right)}\right|\right)\left(\prod_{1 \leq i<j \leq N}\left|1-e^{i\left(\theta_{j}-\theta_{i}\right)}\right|\left|1-e^{-i\left(\theta_{j}-\theta_{i}\right)}\right|\right) \\
& \propto \frac{\left(\prod_{1 \leq i \leq j \leq N}\left|1-e^{-i\left(\theta_{i}+\theta_{j}\right)}\right|\left|1-e^{i\left(\theta_{i}+\theta_{j}\right)}\right|\right)\left(\prod_{1 \leq i<j \leq N}\left|1-e^{i\left(\theta_{j}-\theta_{i}\right)}\right|\left|1-e^{-i\left(\theta_{j}-\theta_{i}\right)}\right|\right)}{\prod_{i=1}^{N}\left|1-e^{2 i \theta_{i}}\right|\left|1-e^{-2 i \theta_{i}}\right|} \\
& \propto e^{-\frac{1}{n} \sum_{n}\left(\sum_{1 \leq i \leq j \leq N}\left(e^{i\left(\theta_{i}+\theta_{j}\right)}+e^{-i\left(\theta_{i}+\theta_{j}\right)}\right)+\sum_{1 \leq i<j \leq N}\left(e^{i\left(\theta_{j}-\theta_{i}\right)}+e^{-i\left(\theta_{j}-\theta_{i}\right)}\right)-\sum_{i=1}^{N}\left(e^{2 i \theta_{i}}+e^{-2 i \theta_{i}}\right)\right)} \\
& \propto e^{-\frac{1}{2 n} \sum_{n}\left(\left(\operatorname{Tr} \mathcal{O}^{n}\right)^{2}-\operatorname{Tr}^{2 n}\right)} \quad(\mathrm{H} .6)
\end{aligned}
$$

where $O$ is the orthogonal matrix in diagonal form

$$
\mathcal{O} \sim\left(\begin{array}{ccccc}
y_{1} & 0 & 0 & \cdots & 0 \\
0 & \frac{1}{y_{1}} & 0 & \cdots & 0 \\
0 & 0 & y_{2} & \cdots & 0 \\
0 & 0 & 0 & \frac{1}{y_{2}} \cdots & 0 \\
\cdots & \cdots & \cdots & \cdots & \cdots \\
0 & 0 & \cdots & y_{N} & 0 \\
0 & 0 & 0 & \cdots & \frac{1}{y_{N}}
\end{array}\right)
$$

In going from the fourth line to the fifth line of (H.6) we have converted the range of the product in the first term in the numerator from $i<j$ to $i \leq j$ (and correspondingly divided out the extra terms); this manipulation was needed in order to rewrite the numerator as a trace after exponentiation. 
As an aside it is interesting to compare the measure (H.6) with the measure for a $\mathrm{U}(2 \mathrm{~N})$ matrix integral, evaluated for a matrix whose eigenvalues happen to fall into complex conjugate pairs as is necessarily the case for an orthogonal matrix. In other words consider a $\mathrm{U}(2 N)$ matrix with eigenvalues $e^{i \alpha_{m}}$ where $\alpha_{1}=\theta_{1}, \alpha_{2}=-\theta_{1}, \alpha_{3}=\theta_{2}, \ldots \alpha_{2 N}=-\theta_{N}$. It is well known that the Haar measure for a Unitary matrix integral is proportional to

$$
\begin{aligned}
\prod_{m \neq n} \mid & 1-e^{-i\left(\alpha_{m}-\alpha_{n}\right)} \mid \\
= & e^{-\frac{1}{n} \sum_{n} \operatorname{Tr} U^{n} \operatorname{Tr} U^{-n}}
\end{aligned}
$$

We note that at leading order in the large $N$ limit, (i.e. at order $N^{2}$ ) the $O(2 N)$ measure (H.6) is the square root of the $\mathrm{U}(2 N)$ measure. At first sub-leading order (i.e. order $N)$, however, the $O(2 N)$ measure has an extra contribution - the terms proportional to $\mathrm{Tr} O^{2 m}$ in the last line of (H.6) that have no analogue in the case of $\mathrm{U}(2 N)$ matrix integrals. The additional terms in the $O(2 N)$ measure come from exponentiating denominator in the 5th line of (H.6). ${ }^{131}$ The physical origin of this term lies in the fact that for every eigenvalue $\theta_{i}$, the $O(2 N)$ matrix automatically has another eigenvalue $-\theta_{i}$; there is no volume factor proportional to $\left|1-e^{2 i \theta_{i}}\right|$ associated with this happenstance. From a more formal mathematical point of view, in (H.10) the trace over the adjoint representation (antisymmetric matrices) of $O(2 N)$

$$
\frac{1}{2}\left((\operatorname{Tr} \mathcal{O})^{2}-\operatorname{Tr} \mathcal{O}^{2}\right)
$$

replaces the trace over the $\mathrm{U}(N)$ adjoint representation

$$
(\operatorname{Tr} U)(\operatorname{Tr} U)^{\dagger}
$$

in (H.7). The order $N$ contribution to the $O(2 N)$ measure will play an important role in our evaluation below.

In the large $N$ limit, $\left(\operatorname{Tr} \mathcal{O}^{n}\right)^{2}$ scales as $N^{2}$ while $\operatorname{Tr} \mathcal{O}^{2 n}$ scales as $N$. For this reason we find it convenient to work below with the order unity variables $\rho_{n}$ by the relations

$$
\left(\operatorname{Tr} \mathcal{O}^{n}\right)^{2}=N^{2} \rho_{n}^{2}, \quad \operatorname{Tr} \mathcal{O}^{2 n}=N \rho_{2 n}
$$

We will now explain how the $O(2 N)$ matrix integrals that appear in the plethystic integrals can be explicitly evaluated in the large $N$ limit. For simplicity we present all details for how this works only for the plethystic integral over scalar Lagrangian; the photon and graviton integrals work in a similar way.

The four-particle scalar partition function is given by,

$$
\begin{aligned}
i_{s}^{(4)}(x, y) & =\frac{1}{24}\left(i_{s}^{4}(x, y)+6 i_{s}^{2}(x, y) i_{s}\left(x^{2}, y^{2}\right)+3 i_{s}^{2}\left(x^{2}, y^{2}\right)+8 i_{s}(x, y) i_{s}\left(x^{3}, y^{3}\right)+6 i_{s}\left(x^{4}, y^{4}\right)\right) \\
i_{s}(x, y) & =\left(1-x^{2}\right) \mathrm{D}(x, y)
\end{aligned}
$$

${ }^{131}$ To see this completely explicitly we note that once we insert the values of $\alpha_{m}$ described above into (H.7) it turns into

$$
\left(\prod_{1 \leq i \leq j \leq N}\left|1-e^{-i\left(\theta_{i}+\theta_{j}\right)}\right|\left|1-e^{i\left(\theta_{i}+\theta_{j}\right)}\right|\right)^{2}\left(\prod_{1 \leq i<j \leq N}\left|1-e^{i\left(\theta_{j}-\theta_{i}\right)}\right|\left|1-e^{-i\left(\theta_{j}-\theta_{i}\right)}\right|\right)^{2} .
$$


The Haar integrand for the scalar case becomes,

$$
\begin{aligned}
i_{s}^{(4)}(x, y)= & \frac{1}{24}\left(\frac{6 \mathrm{D}\left(x^{4}, y^{4}\right)}{\mathrm{D}(x, y)}-6\left(x^{2}-1\right)^{3}\left(x^{2}+1\right) \mathrm{D}(x, y) \mathrm{D}\left(x^{2}, y^{2}\right)+\left(x^{2}-1\right)^{4} \mathrm{D}(x, y)^{3}(\text { H.10 })\right. \\
& \left.-\frac{6 x^{8} \mathrm{D}\left(x^{4}, y^{4}\right)}{\mathrm{D}(x, y)}+\frac{3\left(x^{4}-1\right)^{2} \mathrm{D}\left(x^{2}, y^{2}\right)^{2}}{\mathrm{D}(x, y)}+8\left(x^{2}-1\right)^{2}\left(x^{4}+x^{2}+1\right) \mathrm{D}\left(x^{3}, y^{3}\right)\right)
\end{aligned}
$$

The quantity $\mathrm{D}\left(x^{n}, y^{n}\right)^{m}$ can be exponentiated as follows.

$$
\mathrm{D}\left(x^{n}, y^{n}\right)=e^{\sum_{m} \frac{N \rho_{m n} x^{m n}}{m}}=e^{\sum_{m} \frac{n N \alpha_{m}^{n} \rho_{m} x^{m}}{m}}
$$

where $\alpha_{m}^{n}$ is non-zero only if $n=0 \bmod m$. After putting together (H.6)and (H.10), (4.32) becomes,

$$
I_{s}^{D}(x)=\frac{\int \mathcal{D} \rho_{n} e^{-\frac{1}{2 n}\left(N^{2} \rho_{n}^{2}-N \rho_{2 n}\right)} i_{s}^{(4)}(x, y) / \mathrm{D}(x, y)}{\int \mathcal{D} \rho_{n} e^{-\frac{1}{2 n}\left(N^{2} \rho_{n}^{2}-N \rho_{2 n}\right)}}
$$

where we have defined the measure $\mathcal{D} \rho_{n} \propto \prod_{n} d \rho_{n}$ and we have been careful enough to factor out by the group volume. Each of the 6 terms in (H.10) can now be converted into a Gaussian integral over the variables $\rho_{n}$. For example the first term in $(H .10)$ can be evaluated as

$$
\begin{aligned}
\frac{\int \mathcal{D} \rho_{n} e^{-\frac{1}{2 n}\left(N^{2} \rho_{n}^{2}-N \rho_{2 n}\right)} \mathrm{D}\left(x^{4}, y^{4}\right) / \mathrm{D}(x, y)}{\int \mathcal{D} \rho_{n} e^{-\frac{1}{2 n}\left(N^{2} \rho_{n}^{2}-N \rho_{2 n}\right)}} & =\frac{\int \mathcal{D} \rho_{n} e^{-\frac{1}{2 n}\left(N^{2} \rho_{n}^{2}-2 N \beta_{n}^{2} \rho_{n}-8 N \rho_{n} x^{n} \alpha_{n}^{4}+2 N \rho_{n} x^{n}\right)}}{\int \mathcal{D} \rho_{n} e^{-\frac{1}{2 n}\left(N^{2} \rho_{n}^{2}-2 N \beta_{n}^{2} \rho_{n}\right)}} \\
& =e^{\left(\sum_{n=1}^{\infty} \frac{x^{2 n}\left(4 \alpha_{n}^{4}-1\right)^{2}}{2 n}+\sum_{n=1}^{\infty} \frac{x^{n}\left(4 \alpha_{n}^{4}-1\right) \beta_{n}^{2}}{n}\right)} \\
& =\frac{1}{\left(x^{4}-1\right)^{2}\left(x^{4}+1\right)}
\end{aligned}
$$

where in the second line we have introduced the fictitious counting parameter $\beta_{n}^{2}$ which is non-zero only if $n=0 \bmod 2$, and in going from the second to the third lines in (H.13) we have performed the Gaussian integrals over $\rho_{n}$.

Each of the six terms in (H.10) may be integrated in a similar manner. Summing all the results we find that the integral (H.12) evaluates to

$$
I_{s}^{D}(x)=\frac{1}{\left(1-x^{4}\right)\left(1-x^{6}\right)}=\mathrm{D}
$$

In the case of scalars we believe (and numerical experiments indicate) that (H.14) is correct not just for $D \geq 10$ but in fact for $D \geq 4$.

In order to count photon Lagrangians we need to perform a similar integral.

$$
I_{v}^{D}(x)=\frac{\int \mathcal{D} \rho_{n} e^{-\frac{1}{2 n}\left(N^{2} \rho_{n}^{2}-N \rho_{2 n}\right)} i_{v}^{(4)}(x, y) / \mathrm{D}(x, y)}{\int \mathcal{D} \rho_{n} e^{-\frac{1}{2 n}\left(N^{2} \rho_{n}^{2}-N \rho_{2 n}\right)}}
$$

where $i_{v}^{(4)}(x, y)$ is given by (H.9) and the single letter partition function is given by (4.37),

$$
i_{\mathrm{v}}(x, y)=\left(\left(x-x^{3}\right) \chi_{\square}-\left(1-x^{4}\right)\right) \mathrm{D}(x, y)+1 .
$$


where $\chi_{\square}=N \rho_{1}$. The only difference in this case is the appearance of factors of $\chi_{\square}\left(y^{m}\right)$ or equivalently $N \rho_{m}$ in the Haar integrand due to the multi-particle partition function. This is taken care of in the following manner: we introduce the following fictitious term in the integrand

$$
e^{\sum_{n} \frac{N \gamma_{n}^{1} \rho_{n}}{n}}
$$

where we will set $\gamma_{n}^{1}$ to zero at the end of our computation. The factors of $\chi_{\triangleright}\left(y^{m}\right)$ appearing in the Haar integrand can then be rewritten as derivatives with respect to $\gamma_{n}^{1}$. Operationally, we do the saddle point analysis first and then take the derivatives with respect to $\gamma_{n}^{1}$ to account for the $\chi_{\square}\left(y^{m}\right)$ terms in the Haar integrand. ${ }^{132}$ Following this algorithm and performing a fair amount of algebra we finally obtain

$$
I_{v}^{D}(x)=\frac{x^{4}\left(2 x^{4}+3 x^{2}+2\right)}{x^{10}-x^{6}-x^{4}+1}=x^{4}\left(2 x^{4}+3 x^{2}+2\right) \mathrm{D}
$$

The generalization of this method to the case of the graviton plethystic integral is a straightforward one and we find

$$
\begin{aligned}
I_{T}^{D}(x) & =\frac{\int \mathcal{D} \rho_{n} e^{-\frac{1}{2 n}\left(N^{2} \rho_{n}^{2}-N \rho_{2 n}\right)} i_{T}^{(4)}(x, y) / \mathrm{D}(x, y)}{\int \mathcal{D} \rho_{n} e^{-\frac{1}{2 n}\left(N^{2} \rho_{n}^{2}-N \rho_{2 n}\right)}} \\
& =\frac{\left(x^{8}\left(7+10 x^{2}+10 x^{4}+2 x^{6}-x^{8}+x^{10}\right)\right)}{\left(1-x^{4}-x^{6}+x^{10}\right)} \\
& =x^{8}\left(7+10 x^{2}+10 x^{4}+2 x^{6}-x^{8}+x^{10}\right) \mathrm{D}
\end{aligned}
$$

\section{H.2 $D<10$}

For $D<10$ the results (H.18) and (H.19) are no longer correct. In these dimensions we have not been able to perform the Haar integrals in for the photon or graviton plethystic formulae analytically. We nonetheless have strongly motivated conjectures for the exact results for these integrals using numerical integration as we now explain.

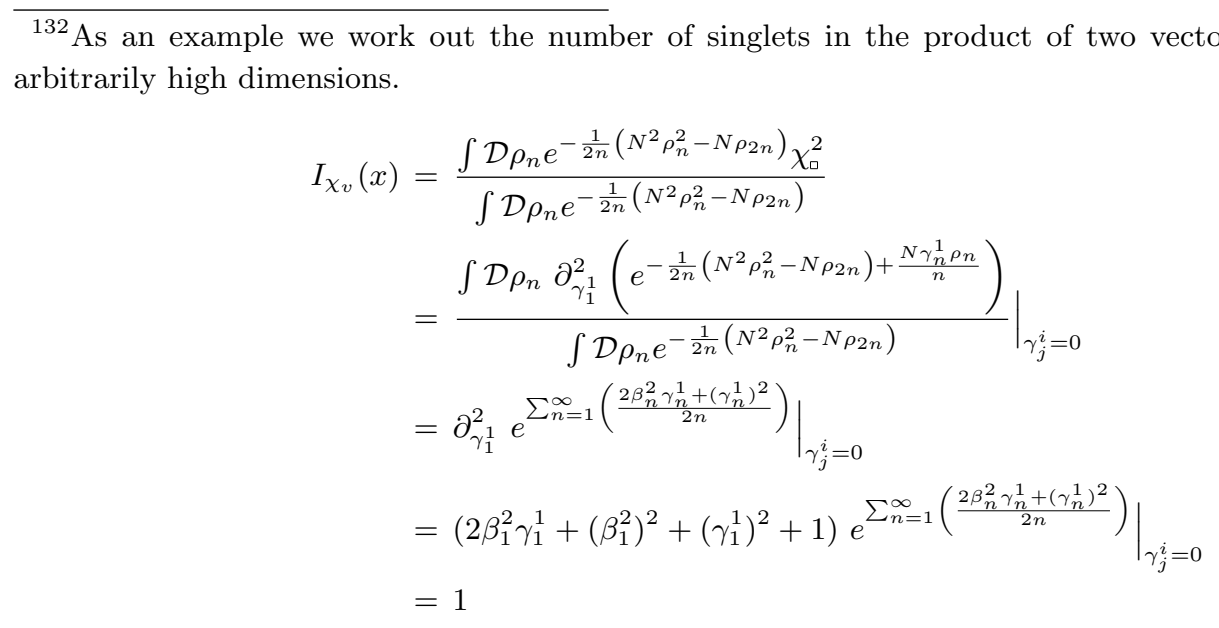

This is consistent with our expectation that only one singlet is there in the tensor product of two vector representation of $\mathrm{SO}(D)$. 
As above we make the change of variables

$$
y_{i}=e^{i \theta_{i}}
$$

As above, the contour integral over $y_{i}$ becomes and angular integral over $\theta_{i} \sim(0,2 \pi)$. The Haar measure becomes,

$$
\begin{aligned}
& \Delta_{e}\left(\theta_{i}\right)=\frac{2^{1-N}\left(\prod_{j=1}^{N}\left(\prod_{i=1}^{j-1} 2\left(\cos \left(\theta_{i}\right)-\cos \left(\theta_{j}\right)\right)\right)\right)^{2}}{(2 \pi)^{N} N !} \\
& \Delta_{o}\left(\theta_{i}\right)=\frac{(-1)^{N}\left(\prod_{k=1}^{N}\left(\cos \left(\theta_{k}\right)-1\right)\right)\left(\prod_{j=1}^{N}\left(\prod_{i=1}^{j-1} 2\left(\cos \left(\theta_{i}\right)-\cos \left(\theta_{j}\right)\right)\right)\right)^{2}}{(2 \pi)^{N} N !}
\end{aligned}
$$

The quantity D changes to

$$
\begin{aligned}
\mathrm{D}_{e}\left(x, \theta_{i}\right) & =\frac{1}{\prod_{i=1}^{N}\left(1-2 x \cos \left(\theta_{i}\right)+x^{2}\right)} \\
\mathrm{D}_{o}\left(x, \theta_{i}\right) & =\frac{1}{(1-x) \prod_{i=1}^{N}\left(1-2 x \cos \left(\theta_{i}\right)+x^{2}\right)}
\end{aligned}
$$

The Haar integral now becomes

$$
I_{j}^{D}(x)=\int_{0}^{2 \pi} \cdots \int_{0}^{2 \pi} \Delta_{e, o}\left(\theta_{i}\right) i_{j}^{(4)}\left(x, \theta_{i}\right) / \mathrm{D}_{e, o}\left(x, \theta_{i}\right)
$$

In order to perform this integral, we first note that we expect the result of the plethystic integral to be a finite linear sum over the partition functions $Z_{\mathbf{1}_{\mathbf{S}}}(x), Z_{\mathbf{2}_{\mathrm{M}}}$ and $Z_{\mathbf{1}_{\mathbf{A}}}(x)$ (see (2.44)) plus (maybe) a finite polynomial accounting for the 'errors' in the plethystic procedure (i.e. the difference between the plethystic partition function and the partition function over S-matrices). Given that each of $Z_{\mathbf{1}_{\mathrm{S}}}(x), Z_{\mathbf{2}_{\mathrm{M}}}$ and $Z_{\mathbf{1}_{\mathrm{A}}}(x)$ equals a polynomial times $\mathrm{D}$ and given that $1 / \mathrm{D}$ is itself a polynomial, it follows, in other words, that we expect the plethystic partition function times $1 / D$ to be a finite polynomial in $x$. Motivated by these observations we multiply the plethystic integrand by $1 / \mathrm{D}$, Taylor series expand the result in $x$ around 0 . The coefficient of every power of $x$ in the result is an integral over $\theta_{i}$. We then evaluate these numerically using the Gauss-Kronrod method. As the numerical integration procedure is very accurate and can be performed very rapidly, we are able to perform this integral up to $x^{26}$. We thus able to verify that the polynomials in $x$ are indeed finite (they terminate) and to evaluate all nonzero coefficients. Our final results are summarized in 5,6 and 7 for $D \leq 10$.

\section{Most general quartic photon Lagrangian}

In this section we will demonstrate that the Lagrangians (5.16) generate all parity even photon S-matrices in every dimension.

All parity even gauge invariant contributions to the Lagrangian that contribute to 4 photon scattering consist of products of derivatives (of arbitrary number) multiplying 4 $F_{\mu \nu}$ fields in such a way that all indices contract so that the Lagrangian is a scalar. 


\section{I.1 Terms with 6 derivatives on $4 F_{\mu \nu}$ are all descendants}

It is very easy to see that every term involving 6 or more derivatives (distributed and contracted in any manner among the $4 F_{\mu \nu}$ operators) is a 'descendant' Lagrangian (i.e. the module elements 'dual' to these Lagrangians are always descendants of more elementary generators). In order to see why this is the case, suppose it were not true. Then there must be a scalar expression built out of $4 F_{\mu \nu} s$ and 6 derivatives in which none of the derivatives contract with each other. It follows that both indices of at least two $F_{a b}$ operators must contract with derivatives. A candidate term of this term might be

$$
\partial_{a} F_{\mu \nu} \partial_{\mu} F_{a b} \partial_{b} \partial_{\nu} \partial^{p} F_{m n} \partial^{m} F_{p n}
$$

in which the indices of the first two field strength operators are both contracted with derivatives. In order to see that the term above is trivial we use the Bianchi identity

$$
\partial_{a} F_{\mu \nu}=-\partial_{\mu} F_{\nu a}-\partial_{\nu} F_{a \mu}
$$

to re-express the first field strength in (I.1) as a sum of two other terms. This gives us a sum of terms, each of which is a product of four field strengths. Note, however, that both of these terms have a pair of derivatives with contracted indices, and so both terms are descendants as we wanted to show.

The reader can easily convince herself that exactly the same argument can be made whenever two separate field strength operators have both their indices contracted with derivatives. Let the two field strengths of this form be the 'first' and the second $F_{\mu \nu}$ operators in the expression. The two derivatives that contract with the second $F_{\mu \nu}$ must act on distinct $F_{\alpha \beta}$ fields (else the expression would vanish by the antisymmetry of $F_{a b}$ ). Moreover neither of these derivatives can act on the second field itself (else the expression would vanish by the equations of motion). An integration by parts can be used to ensure that none of the derivatives act on the 'fourth' $F_{\mu \nu}$. With this convention it follows that one of the two derivatives that contracts with the second $F_{\mu \nu}$ must act on the 'third' $F_{\mu \nu}$ while the second derivative must act on the first $F_{\mu \nu}$. We can now replace the expression involving the derivative acting on the first $F_{\mu \nu}$ by two different terms via the Bianchi identity. A moment's consideration will convince the reader that both these terms are descendants.

\section{I.2 Terms with 4 derivatives on $4 F_{\mu \nu}$ are also all descendants}

Let us now turn to terms involving four derivatives acting on the four $F_{\mu \nu}$ operators. The reader can quickly convince herself that there are five terms of this sort that are not obviously trivial. These terms are

$$
\begin{aligned}
& T_{1}^{4}=\partial_{\delta} \partial_{\nu} F_{\alpha a} F_{\beta a} \partial_{\alpha} \partial_{\beta} F_{\nu \beta} F_{\delta b} \\
& T_{2}^{4}=\partial_{\nu} F_{\alpha a} \partial_{\delta} F_{\beta a} \partial_{\alpha} F_{\nu b} \partial_{\beta} F_{\delta b} \\
& T_{3}^{4}=\partial_{\delta} \partial_{\gamma} F_{a \alpha} F_{a \beta} \partial_{\alpha} F_{b \gamma} \partial_{\beta} F_{b \delta} \\
& T_{4}^{4}=\partial_{\beta} \partial_{d} F_{a b} F_{b c} \partial_{\alpha} \partial_{a} F_{c d} F_{\alpha \beta} \\
& T_{5}^{4}=\partial_{\gamma} \partial_{\alpha} F_{a b} \partial_{\delta} \partial_{\beta} F_{b a} F_{\alpha \beta} F_{\gamma \delta}
\end{aligned}
$$


(Any other expression that the reader may care to write down can be manipulated into one of the five forms above up to total derivatives - without, at this stage, the use of Bianchi identities).

It is now possible to employ Bianchi identities to find relations between the structures $T_{i}^{4}(i=1 \rightarrow 5)$. The relations we obtain turn out to be strong enough to allow us to deduce that each of the terms listed in (I.2) actually are actually trivial. The algebra involved in these demonstrations is lengthy — so we only report one sample computation

$$
\begin{aligned}
T_{1}^{4} & =k_{\alpha}^{3} F_{\alpha a}^{1} k_{\beta}^{3} F_{\beta a}^{2} k_{\gamma}^{1} F_{\gamma b}^{3} k_{\delta}^{1} F_{\delta b}^{4} \\
& =-k_{\alpha}^{3} F_{\gamma \alpha}^{1} k_{\beta}^{3} F_{\beta a}^{2} k_{a}^{1} F_{\gamma b}^{3} k_{\delta}^{1} F_{\delta b}^{4}-k_{\alpha}^{3} F_{a \gamma}^{1} k_{\beta}^{3} F_{\beta a}^{2} k_{\alpha}^{1} F_{\gamma b}^{3} k_{\delta}^{1} F_{\delta b}^{4} \\
& =-k_{\alpha}^{3} F_{\gamma \alpha}^{1} k_{\beta}^{3} F_{\beta a}^{2} k_{a}^{1} F_{\gamma b}^{3} k_{\delta}^{1} F_{\delta b}^{4}+I_{\mathrm{desc}} \\
& =k_{\gamma}^{3} F_{\gamma \alpha}^{1} k_{\beta}^{3} F_{\beta a}^{2} k_{a}^{1} F_{b \alpha}^{3} k_{\delta}^{1} F_{\delta b}^{4}+k_{b}^{3} F_{\gamma \alpha}^{1} k_{\beta}^{3} F_{\beta a}^{2} k_{a}^{1} F_{\alpha \gamma}^{3} k_{\delta}^{1} F_{\delta b}^{4}+I_{\mathrm{desc}} \\
& =k_{\alpha}^{3} F_{\gamma \alpha}^{1} k_{\beta}^{3} F_{\beta a}^{2} k_{a}^{1} F_{\gamma b}^{3} k_{\delta}^{1} F_{\delta b}^{4}+k_{b}^{3} F_{\gamma \alpha}^{1} k_{\beta}^{3} F_{\beta a}^{2} k_{a}^{1} F_{\alpha \gamma}^{3} k_{\delta}^{1} F_{\delta b}^{4}+I_{\mathrm{desc}} \\
& =k_{\alpha}^{3} F_{\gamma \alpha}^{1} k_{\beta}^{3} F_{\beta a}^{2} k_{a}^{1} F_{\gamma b}^{3} k_{\delta}^{1} F_{\delta b}^{4}-k_{b}^{2} F_{\gamma \alpha}^{1} k_{\beta}^{3} F_{\beta a}^{2} k_{a}^{1} F_{\alpha \gamma}^{3} k_{\delta}^{1} F_{\delta b}^{4}+I_{\mathrm{desc}} \\
\therefore T_{1}^{4} & \sim-k_{b}^{2} F_{\gamma \alpha}^{1} k_{\beta}^{3} F_{\beta a}^{2} k_{a}^{1} F_{\alpha \gamma}^{3} k_{\delta}^{1} F_{\delta b}^{4}+I_{\mathrm{desc}} \sim \widetilde{I}_{\mathrm{desc}}
\end{aligned}
$$

In deriving this we have used Bianchi identity between the first $k^{1}$ and $F^{1}$ in the second line. In the fourth line we use Bianchi identity between first $k^{3}$ and $F^{3}$. The structure in the sixth line is due to momentum conservation and antisymmetry of $F_{a b}$. In the final step, to go from $I_{\text {desc }}$ to $\widetilde{I}_{\text {desc }}$, we have used Bianchi identity between $k^{2}$ and $F^{2}$.

Similar manipulations can be used to prove that all of the $T_{i}^{4} \mathrm{~s}$ are descendants of structures with no more than two derivatives on $F_{\mu \nu}$. It follows that there is no Lagrangian structure built out of four field strengths and four derivatives that generates a 'primary' S-matrix.

\section{I.3 Primary structures with two derivatives on four field strengths}

The situation is a bit more complicated with terms involving two derivatives of the four field strengths. By using the equivalence of terms that differ by total derivatives, the reader can convince herself that there are fourteen naively inequivalent structures at this order. 
They are

$$
\begin{aligned}
& T_{1}^{2}=\partial_{b} F_{\beta a} \partial_{a} F_{\alpha b} F_{\theta \alpha} F_{\theta \beta} \\
& T_{2}^{2}=\partial_{b} F_{\beta a} \partial_{a} F_{\beta b} F_{\mu \nu} F_{\mu \nu} \\
& T_{3}^{2}=\partial_{b} F_{\beta a} \partial_{a} F_{\alpha b} F_{\theta \beta} F_{\theta \alpha} \\
& T_{4}^{2}=F_{\beta a} \partial_{a} F_{\alpha b} \partial_{b} F_{\theta \alpha} F_{\theta \beta} \\
& T_{5}^{2}=F_{\beta a} \partial_{a} F_{\beta b} \partial_{b} F_{\mu \nu} F_{\mu \nu} \\
& T_{6}^{2}=F_{\beta a} \partial_{a} F_{\alpha b} \partial_{b} F_{\theta \beta} F_{\theta \alpha} \\
& T_{7}^{2}=\partial_{\beta} F_{b a} F_{\theta \beta} \partial_{a} F_{b \alpha} F_{\theta \alpha} \\
& T_{8}^{2}=F_{\alpha a} F_{\beta b} \partial_{a} F_{\alpha \theta} \partial_{b} F_{\beta \theta} \\
& T_{9}^{2}=F_{\alpha a} F_{\beta b} \partial_{b} \partial_{a} F_{\alpha \theta} F_{\beta \theta} \\
& T_{10}^{2}=F_{b \alpha} F_{a \alpha} \partial_{b} F_{\mu \nu} \partial_{a} F_{\mu \nu} \\
& T_{11}^{2}=F_{b \alpha} F_{a \alpha} \partial_{a} \partial_{b} F_{\mu \nu} F_{\mu \nu} \\
& T_{12}^{2}=F_{\alpha a} F_{\beta b} \partial_{a} F_{\beta \theta} \partial_{b} F_{\alpha \theta} \\
& T_{13}^{2}=F_{\alpha a} F_{\beta b} \partial_{a} \partial_{b} F_{\beta \theta} F_{\alpha \theta} \\
& T_{14}^{2}=F_{a b} \partial_{a} F_{\mu \nu} \partial_{b} F_{\nu \rho} F_{\rho \mu}
\end{aligned}
$$

$T_{1}^{2}$ and $T_{3}^{2}$ are equivalent up to re-labelling.

Once again these naively independent structures are not really all distinct; once again Bianchi identities may be used to relate these 14 structures. It turns out that Bianchi identities generates 13 non-trivial identities between the structures listed in (I.4). These identities can use used to relate each of these structures to a single independent term which we choose to be

$$
T_{I}^{2}=F_{a b} \partial_{a} F_{\mu \nu} \partial_{b} F_{\nu \rho} F_{\rho \mu}
$$

Once again the algebra to establish these results is to lengthy to record in entirety; once again we only present some (in this case 2) sample manipulations.

$$
\begin{aligned}
T_{1}^{2} & =k_{a}^{2} F_{\beta a}^{1} k_{b}^{1} F_{\alpha b}^{2} F_{\theta \alpha}^{3} F_{\theta \beta}^{4} \\
& \equiv k_{a}^{1} F_{\beta a}^{2} k_{b}^{2} F_{\alpha b}^{1} F_{\theta \alpha}^{3} F_{\theta \beta}^{4} \\
& =k_{b}^{2} F_{\alpha b}^{1} k_{a}^{1} F_{\beta a}^{2} F_{\theta \alpha}^{3} F_{\theta \beta}^{4} \\
& =T_{3}^{2} \\
& =-k_{a}^{2} F_{a b}^{1} k_{\beta}^{1} F_{\alpha b}^{2} F_{\theta \beta}^{3} F_{\theta \alpha}^{4}-k_{a}^{2} F_{b \beta}^{1} k_{a}^{1} F_{\alpha b}^{2} F_{\theta \beta}^{3} F_{\theta \alpha}^{4} \\
& =-k_{a}^{2} F_{b a}^{1} k_{\beta}^{1} F_{\theta \beta}^{3} F_{b \alpha}^{2} F_{\theta \alpha}^{4}+\mathcal{O}\left(\operatorname{desc}\left(\operatorname{Tr} F^{4}\right)\right) \\
& =-k_{a}^{2} F_{b a}^{1} k_{\beta}^{1} F_{\theta \beta}^{3} F_{b \alpha}^{2} F_{\theta \alpha}^{4}+\mathcal{O}\left(\operatorname{desc}\left(\operatorname{Tr} F^{4}\right)\right) \\
& =k_{b}^{2} F_{b a}^{1} k_{\beta}^{1} F_{\theta \beta}^{3} F_{\alpha a}^{2} F_{\theta \alpha}^{4}+k_{\alpha}^{2} F_{b a}^{1} k_{\beta}^{1} F_{\theta \beta}^{3} F_{a b}^{2} F_{\theta \alpha}^{4}+\mathcal{O}\left(\operatorname{desc}\left(\operatorname{Tr} F^{4}\right)\right) \\
& =k_{a}^{2} F_{a b}^{1} k_{\beta}^{1} F_{\theta \beta}^{3} F_{\alpha b}^{2} F_{\theta \alpha}^{4}+k_{\alpha}^{2} F_{\theta \alpha}^{4} k_{\beta}^{1} F_{\theta \beta}^{3} F_{b a}^{1} F_{a b}^{2}+\mathcal{O}\left(\operatorname{desc}\left(\operatorname{Tr} F^{4}\right)\right) \\
& =k_{a}^{2} F_{a b}^{1} k_{\beta}^{1} F_{\theta \beta}^{3} F_{\alpha b}^{2} F_{\theta \alpha}^{4}+T_{10}^{2}+\mathcal{O}\left(\operatorname{desc}\left(\operatorname{Tr} F^{4}\right)\right) \\
\therefore T_{1}^{2} & =T_{3}^{2} \sim T_{10}^{2}+\mathcal{O}\left(\operatorname{desc}\left(\operatorname{Tr} F^{4}\right)\right)
\end{aligned}
$$


The steps in the manipulation are as follows. In the second line we relabel $(1 \leftrightarrow 2)$ to establish the fact that $T_{1}^{2} \sim T_{3}^{2}$ in momentum space. In the fifth line, we use Bianchi identity corresponding to particle 1 . In the eighth line we use Bianchi identity corresponding to particle 2. Equating the seventh and tenth line we obtain the final identity. Hence we have the Lagrangian term $T_{1}^{2}$ is identical to $T_{10}^{2}$ up to descendants of four photon Lagrangians of derivative order 4 .

Let us now look at a second example

$$
\begin{aligned}
T_{6}^{2} & =k_{a}^{2} F_{\beta a}^{1} k_{b}^{3} F_{\alpha b}^{2} F_{\theta \beta}^{3} F_{\theta \alpha}^{4} \\
& =-k_{\alpha}^{2} F_{\beta a}^{1} k_{b}^{3} F_{b a}^{2} F_{\theta \beta}^{3} F_{\theta \alpha}^{4}+\mathcal{O}\left(\operatorname{desc}\left(\operatorname{Tr} F^{4}\right)\right) \\
& =-k_{\alpha}^{2} F_{\theta \alpha}^{1} k_{b}^{3} F_{b a}^{2} F_{\beta a}^{4} F_{\theta \beta}^{3}+\mathcal{O}\left(\operatorname{desc}\left(\operatorname{Tr} F^{4}\right)\right) \\
& =-k_{\alpha}^{2} F_{\theta \alpha}^{1} k_{b}^{3} F_{a b}^{2} F_{\beta \theta}^{3} F_{\beta a}^{4}+\mathcal{O}\left(\operatorname{desc}\left(\operatorname{Tr} F^{4}\right)\right) \\
\therefore T_{6}^{2} & \sim \mathcal{O}\left(\operatorname{desc}\left(\operatorname{Tr} F^{4}\right)\right)
\end{aligned}
$$

where we have used Bianchi identity corresponding to the second particle and re-labelling of $(1 \leftrightarrow 4)$. In this way all the Lagrangian structures can be represented in terms of $T_{10}^{2}$ and descendants of $\operatorname{Tr} F^{4}$ and $\left(\operatorname{Tr} F^{2}\right)^{2}$.

\section{J Most general parity even photon S-matrix that grows no faster than $s^{2}$ in the Regge limit}

In this subsection we will classify all local 4 photon S-matrices that grow no faster than $s^{2}$ in the Regge limit for every choice of polarization vectors. Consider the most general S-matrix from (5.20).

$$
\begin{aligned}
& S^{E_{\mathbf{3}, 1}^{(1)}}=\mathcal{F}^{\mathbf{3}, 1}(t, u)\left(-8 s^{2} e_{\mathbf{3}, 1}^{(1)}+8 s^{2} e_{\mathbf{3}, 2}^{(1)}-8 s^{2} e_{\mathbf{S}}\right), \\
& S^{E_{\mathbf{3}, 1}^{(2)}}=\mathcal{F}^{\mathbf{3}, 1}(u, s)\left(-8 t^{2} e_{\mathbf{3}, 1}^{(2)}+8 t^{2} e_{\mathbf{3}, 2}^{(2)}-8 t^{2} e_{\mathbf{S}}\right), \\
& S^{E_{\mathbf{3}, 1}^{(3)}}=\mathcal{F}^{\mathbf{3}, 1}(s, t)\left(-8 u^{2} e_{\mathbf{3}, 1}^{(3)}+8 u^{2} e_{\mathbf{3}, 2}^{(3)}-8 u^{2} e_{\mathbf{S}}\right), \\
& S^{E_{\mathbf{3}, 2}^{(1)}}=\mathcal{F}^{\mathbf{3}, 2}(t, u)\left(-2\left(u^{2} e_{\mathbf{3}, 1}^{(2)}+t^{2} e_{\mathbf{3}, 1}^{(3)}\right)+2\left(u(s-t) e_{\mathbf{3}, 2}^{(2)}+t(s-u) e_{\mathbf{3}, 2}^{(3)}\right)-2\left(t^{2}+u^{2}\right) e_{\mathbf{S}}\right), \\
& S^{E_{\mathbf{3}, 2}^{(2)}}=\mathcal{F}^{\mathbf{3}, 2}(u, s)\left(-2\left(s^{2} e_{\mathbf{3}, 1}^{(3)}+u^{2} e_{\mathbf{3}, 1}^{(1)}\right)+2\left(s(t-u) e_{\mathbf{3}, 2}^{(3)}+u(t-s) e_{\mathbf{3}, 2}^{(1)}\right)-2\left(u^{2}+s^{2}\right) e_{\mathbf{S}}\right), \\
& S^{E_{\mathbf{3}, 2}^{(3)}}=\mathcal{F}^{\mathbf{3}, 2}(s, t)\left(-2\left(t^{2} e_{\mathbf{3}, 1}^{(1)}+s^{2} e_{\mathbf{3}, 1}^{(2)}\right)+2\left(t(u-s) e_{\mathbf{3}, 2}^{(1)}+s(u-t) e_{\mathbf{3}, 2}^{(2)}\right)-2\left(s^{2}+t^{2}\right) e_{\mathbf{S}}\right), \\
& S^{E_{\mathbf{S}}}=\left(\mathcal{F}^{E_{\mathbf{S}}}(t, u)\right)\left(3 s t u\left(e_{\mathbf{3}, 2}^{(1)}+e_{\mathbf{3}, 2}^{(2)}+e_{\mathbf{3}, 2}^{(3)}-2 e_{\mathbf{S}}\right)\right) .
\end{aligned}
$$

For the purpose of analyzing the Regge growth, let us rearrange the terms in the Smatrix (5.20) in the following way. We consider terms in the S-matrix which are pro- 
portional to the bare generator $e$ 's from each of the local generators $E$ 's.

$$
\begin{aligned}
& \left.S^{E_{\mathbf{3}, 1}}\right|_{e_{\mathbf{3}, 1}}=-8 \mathcal{F}^{\mathbf{3}, 1}(t, u) s^{2} e_{\mathbf{3}, 1}^{(1)}-8 \mathcal{F}^{\mathbf{3}, 1}(u, s) t^{2} e_{\mathbf{3}, 1}^{(2)}-8 \mathcal{F}^{\mathbf{3}, 1}(s, t) u^{2} e_{\mathbf{3}, 1}^{(3)}, \\
& \left.S^{E_{\mathbf{3}, 2}}\right|_{e_{\mathbf{3}, 1}}=-2\left(\left(\mathcal{F}^{\mathbf{3}, 2}(u, s) u^{2}+\mathcal{F}^{\mathbf{3}, 2}(s, t) t^{2}\right) e_{\mathbf{3}, 1}^{(1)}+\left(\mathcal{F}^{\mathbf{3}, 2}(t, u) u^{2}+\mathcal{F}^{\mathbf{3}, 2}(s, t) s^{2}\right) e_{\mathbf{3}, 1}^{(2)}\right. \\
& \left.+\left(\mathcal{F}^{\mathbf{3}, 2}(t, u) t^{2}+\mathcal{F}^{\mathbf{3}, 2}(u, s) s^{2}\right) e_{\mathbf{3}, 1}^{(3)}\right), \\
& \left.S^{E_{\mathbf{S}}}\right|_{e_{3,1}}=0 . \\
& \left.S\right|_{e_{\mathbf{3}, 1}}=S^{E_{\mathbf{3}, 1}}+S^{E_{\mathbf{3}, 2}}+\left.S^{E_{\mathbf{S}}}\right|_{e_{\mathbf{3}, 1}} .
\end{aligned}
$$

For the terms proportional to $e_{\mathbf{3}, 2}^{(1)}$ and its permutations, we obtain,

$$
\begin{aligned}
\left.S^{E_{\mathbf{3}, 1}}\right|_{\mathbf{e}_{\mathbf{3}, 2}}=8 & \mathcal{F}^{E_{\mathbf{3}, 1}}(t, u) s^{2} e_{\mathbf{3}, 2}^{(1)}+8 \mathcal{F}^{E_{\mathbf{3}, 1}}(s, u) t^{2} e_{\mathbf{3}, 2}^{(2)}+8 \mathcal{F}^{E_{\mathbf{3}, 1}}(t, s) u^{2} e_{\mathbf{3}, 2}^{(3)} \\
\left.S^{E_{\mathbf{3}, 2}}\right|_{e_{\mathbf{3}, 2}}=2( & \left(\mathcal{F}^{E_{\mathbf{3}, 2}}(s, u) u(t-s)+\mathcal{F}^{E_{\mathbf{3}, 2}}(t, s) t(u-s)\right) e_{\mathbf{3}, 2}^{(1)} \\
& +\left(\mathcal{F}^{E_{\mathbf{3}, 2}}(t, u) u(s-t)+\mathcal{F}^{E_{\mathbf{3}, 2}}(t, s) s(u-t)\right) e_{\mathbf{3}, 2}^{(2)} \\
& \left.+\left(\mathcal{F}^{E_{\mathbf{3}, 2}}(t, u) t(s-u)+\mathcal{F}^{E_{\mathbf{3}, 2}}(s, u) s(t-u)\right) e_{\mathbf{3}, 2}^{(3)}\right) \\
\left.S^{E_{\mathbf{S}}}\right|_{e_{\mathbf{3}, 2}}= & \mathcal{F}^{E_{\mathbf{S}}}(t, u)\left(3 s t u\left(e_{\mathbf{3}, 2}^{(1)}+e_{\mathbf{3}, 2}^{(2)}+e_{\mathbf{3}, 2}^{(3)}\right)\right) \\
\left.S\right|_{e_{\mathbf{3}, 2}}= & S^{E_{\mathbf{3}, 1}}+S^{E_{\mathbf{3}, 2}}+\left.S^{E_{\mathbf{S}}}\right|_{e_{\mathbf{3}, 2}}
\end{aligned}
$$

And finally, for terms proportional to $e_{\mathbf{S}}$,

$$
\begin{aligned}
\left.S^{E_{\mathbf{3}, 1}}\right|_{e_{\mathbf{S}}} & =\left(-8 \mathcal{F}^{E_{\mathbf{3}, 1}}(t, u) s^{2}-8 \mathcal{F}^{E_{\mathbf{3}, 1}}(s, u) t^{2}-8 \mathcal{F}^{E_{\mathbf{3}, 1}}(t, s) u^{2}\right) e_{\mathbf{S}} \\
\left.S^{E_{\mathbf{3}, 2}}\right|_{e_{\mathbf{S}}} & =-2\left(\mathcal{F}^{E_{\mathbf{3}, 2}}(s, u)\left(u^{2}+s^{2}\right)+\mathcal{F}^{E_{\mathbf{3}, 2}}(t, s)\left(s^{2}+t^{2}\right)+\mathcal{F}^{E_{\mathbf{3}, 2}}(t, u)\left(t^{2}+u^{2}\right)\right) e_{\mathbf{S}} \\
\left.S^{E_{\mathbf{S}}}\right|_{e_{\mathbf{S}}} & =-6 s t u \mathcal{F}^{E_{\mathbf{S}}}(t, u) e_{\mathbf{S}} \\
\left.S\right|_{e_{\mathbf{S}}} & =S^{E_{\mathbf{3}, 1}}+S^{E_{\mathbf{3}, 2}}+\left.S^{E_{\mathbf{S}}}\right|_{e_{\mathbf{S}}} .
\end{aligned}
$$

Let us first study terms proportional to $e_{\mathbf{3}, 1}^{(3)}$ of the S-matrix (These are given by the last line in (J.2)). Provided that $D \geq 4$ the condition that the S-matrix grow no faster than $s^{2}$ is only met provided the same condition holds independently for the coefficients of $e_{\mathbf{3}, 1}^{(1)}$ and $e_{\mathbf{3}, 1}^{(2)}$. Our condition is thus simply that

$$
-\left(8 u^{2} \mathcal{F}^{E_{\mathbf{3}, 1}}(t, s)+2 s^{2} \mathcal{F}^{E_{\mathbf{3}, 2}}(s, u)+2 t^{2} \mathcal{F}^{E_{\mathbf{3}, 2}}(t, u)\right)
$$

(together with the two crossing related expressions) grow no faster than $s^{2}$ in the Regge limit.

If we assume that $\mathcal{F}^{E_{3,1}}$ and $\mathcal{F}^{E_{3,2}}$ are polynomials then the expression in (J.5) is a polynomial of degree 2 or greater. Moreover it is symmetric under interchange of $s$ and $t$. The only polynomials that meet these conditions and still do not grow faster than $s^{2}$ are $s^{2}+t^{2}, s t, s^{2} t+t^{2} s$ and $s^{2} t^{2}$. Now if the polynomial in (J.5) were to evaluate to $s^{2} t^{2}$ then the (permutation related) polynomial that occurs in the bracket of the first line of (J.2) 
(proportional to $e_{1}^{(1)}$ ) would evaluate to $s^{2} u^{2}$. As this expression grows faster than $s^{2}$ and so is disallowed. We conclude that the expression in (J.5) must be a linear combination of $s^{2}+t^{2}, s t$ and $s^{2} t+t^{2} s$.

Let us now turn to the result of (J.4) above. Notice that the part of this answer that depends on $\mathcal{F}^{E_{\mathbf{3}, 1}}$ and $\mathcal{F}^{E_{\mathbf{3}, 2}}$ is proportional to the term in (J.5) completely symmetrized (i.e. is proportional to the sum of the three brackets in the last three lines of (J.2)). Given the conditions of the last paragraph, this term automatically grows no faster than $s^{2}$ in the Regge limit. It follows that (J.4) grows no faster than $s^{2}$ in the Regge limit provided the same is true of $-s t u \mathcal{F}^{E_{\mathbf{S}}}(t, u)$. This condition immediately forces $\mathcal{F}^{E_{\mathbf{S}}}(t, u)$ to be a constant.

Finally, let us turn to the expression in (J.3) above. The coefficient of $e_{\mathbf{3}, 2}^{(3)}$ in that expression is given by

$$
8 u^{2} \mathcal{F}^{E_{\mathbf{3}, 1}}(t, s)+2 s(t-u) \mathcal{F}^{E_{\mathbf{3}, 2}}(s, u)+2 t(s-u) \mathcal{F}^{E_{\mathbf{3}, 1}}(t, u)+3 s t u \mathcal{F}^{E_{\mathbf{S}}}(t, u)
$$

As we now know that $\mathcal{F}^{E_{\mathbf{S}}}(t, u)$ is a constant, the term proportional to $\mathcal{F}^{E_{\mathbf{S}}}(t, u)$ in this expression is proportional to $s t u$ and so automatically grows no faster than $s^{2}$ in the Regge limit. In order that our S-matrix grow no faster than $s^{2}$ at fixed $t$, it must be that the same is true of the expression

$$
8 u^{2} \mathcal{F}^{E_{\mathbf{3}, 1}}(t, s)+2 s(t-u) \mathcal{F}^{E_{\mathbf{3}, 2}}(s, u)+2 t(s-u) \mathcal{F}^{E_{\mathbf{3}, 2}}(t, u)
$$

By repeating the reasoning in the paragraph under (J.5) it must be that (J.7), like (J.5), is a linear combination of the polynomials $s^{2}+t^{2}$, st, and $s t u$.

In summary we require that the expressions in (J.5) and (J.7) must simultaneously be (possibly different) linear combinations of the three polynomials listed above. If $\mathcal{F}^{E_{\mathbf{3}, 1}}$ and $\mathcal{F}^{E_{3,2}}$ are constants, then both (J.5) and (J.7) are automatically linear combinations of $s^{2}+t^{2}$ and st. The only other possibility for $\mathcal{F}^{E_{\mathbf{3}, 1}}$ and $\mathcal{F}^{E_{\mathbf{3}, 2}}$ is that they are proportional to the (unique symmetric degree one) polynomial $s+t$. If we suppose that $\mathcal{F}^{E_{3,1}}(s, t)=a(s+t)$ and that $\mathcal{F}^{E_{\mathbf{3}, 2}}(s, t)=b(s+t)$ then (J.5) evaluates to

$$
-4 a u^{3}-b\left(s^{2} t+t^{2} s\right)
$$

The condition that this expression grow no faster than $s^{2}$ in the Regge limit sets $a=0$. With this condition (J.7) evaluates to

$$
-s t(s-u)-s t(t-u)=-3 s t u
$$

So $b$ is allowed to be non-zero. In conclusion, the most general photon S-matrix that grows no faster than $s^{2}$ is given by (5.20) with the momenta polynomials specified by

$$
\mathcal{F}^{E_{\mathbf{3}, 1}}(s, t)=a_{1}, \quad \mathcal{F}^{E_{\mathbf{3}, 2}}(s, t)=b_{1}+b_{2}(s+t), \quad \mathcal{F}^{E_{\mathbf{S}}}(s, t)=c_{1}
$$

where $a_{1}, b_{1}, b_{2}$ and $c_{1}$ are all arbitrary constants.

\section{K Explicit S-matrices for four graviton scattering}

In this appendix we provide a completely explicit listing of gravitational S-matrices in every dimension. 


\section{K.1 $\quad D \geq 8$}

When $D \geq 84$ gravity scattering is necessarily parity even. In these dimensions the generators of the local S-matrix module are $G_{\mathbf{S}, 1}=\chi_{6}$ (see (4.20) and (4.25)) along with $G_{\mathbf{3}, 1}, \ldots G_{\mathbf{3}, 8}, G_{\mathbf{3}_{\mathbf{A}}}$ and $G_{\mathbf{S}, 2}($ see $(6.16))$.

It is relatively straightforward to list the $S$ matrices generated by $G_{\mathbf{3}, 1}, \ldots G_{\mathbf{3}, 8}, G_{\mathbf{3}_{\mathbf{A}}}$, $G_{\mathbf{S}, 2}$ as well as the Lagrangians that generate these S-matrices. It is also straightforward to list the S-matrices generated by $G_{\mathbf{S}, 1}$. As explained in subsection 6.1.1, however, the fact that the Lagrangian $G_{\mathbf{S}, 1}$ has only three factors of the Riemann tensor complicates the listing of Lagrangians dual to these S-matrices. As explained in subsection 6.1.1, one can find a solution to this problem by adopting an alternate view of the S-matrix module; a view in which we include generator $G_{\mathbf{3}, 9}$ instead of $G_{\mathbf{S}, 1}$ but the module is now not freely generated but is instead subject to the relations (6.9).

In this subsection we first perform the simple part of our listing. We list the S-matrices generated by $G_{\mathbf{3}, 1}, \ldots G_{\mathbf{3}, 8}, G_{\mathbf{3}_{\mathbf{A}}}, G_{\mathbf{S}, 2}$. After this is done we return to question of listing the Lagrangians that generate the 'descendant' S-matrices of $G_{\mathbf{S}, 1}$.

- The S-matrix corresponding to $G_{\mathbf{3}, 1}$ in (6.17) is specified by the polynomial $\mathcal{F}^{G_{3,1}}(t, u)$ which exhibits a $\mathbb{Z}_{2}$ symmetry $(t \leftrightarrow u)$. In equations,

$$
\begin{aligned}
S^{G_{3,1}}= & \frac{1}{4}\left(\mathcal { F } ^ { G _ { 3 , 1 } } ( t , u ) \left[\left(p_{p}^{1} \epsilon_{q}^{1}-p_{q}^{1} \epsilon_{p}^{1}\right)\left(p_{p}^{2} \epsilon_{q}^{2}-p_{q}^{2} \epsilon_{p}^{2}\right)\left(p_{r}^{3} \epsilon_{s}^{3}-p_{s}^{3} \epsilon_{r}^{3}\right)\left(p_{r}^{4} \epsilon_{s}^{4}-p_{s}^{4} \epsilon_{r}^{4}\right)\right.\right. \\
& \left.\times\left(p_{a}^{1} \epsilon_{b}^{1}-p_{b}^{1} \epsilon_{a}^{1}\right)\left(p_{a}^{2} \epsilon_{b}^{2}-p_{b}^{2} \epsilon_{a}^{2}\right)\left(p_{c}^{3} \epsilon_{d}^{3}-p_{d}^{3} \epsilon_{c}^{3}\right)\left(p_{c}^{4} \epsilon_{d}^{4}-p_{d}^{4} \epsilon_{c}^{4}\right)\right] \\
& \left.+\mathcal{F}^{G_{3,1}}(s, u)[3 \leftrightarrow 2]+\mathcal{F}^{G_{3,1}}(s, t)[2 \leftrightarrow 4]\right) .
\end{aligned}
$$

The most general descendant which gives rise to S-matrix in (K.1) is given by,

$$
L^{G_{3,1}}=\sum_{m, n}\left(\mathcal{F}^{G_{3,1}}\right)_{m, n} 2^{m+n}\left(\prod_{i=1}^{m} \prod_{j=1}^{n}\left(\partial_{\mu_{i}} \partial_{\nu_{j}} R_{a b p q}\right) R_{b a q p}\left(\partial^{\mu_{i}} R_{c d r s}\right)\left(\partial^{\nu_{j}} R_{d c s r}\right)\right) .
$$

We have defined the momenta polynomials as,

$$
\mathcal{F}^{G_{3,1}}(t, u)=\sum_{m, n}\left(\mathcal{F}^{G_{3,1}}\right)_{m, n} t^{m} u^{n} .
$$

In order to see the fact that Lagrangian (K.2) results in the S-matrix (K.1), we note that

$$
R_{a b p q} R_{b a q p} R_{c d r s} R_{d c s r}
$$

linearizes to give $\operatorname{Tr}\left(F^{1} F^{2}\right) \operatorname{Tr}\left(F^{3} F^{4}\right) \operatorname{Tr}\left(F^{1} F^{2}\right) \operatorname{Tr}\left(F^{3} F^{4}\right)$ plus permutations. Once linearized, it is clear that the structure has extra $\mathbb{Z}_{2}$ symmetry of 1 to 2 exchange. The descendant Lagrangian (K.2) therefore linearizes to,

$$
\begin{aligned}
L^{G_{3,1}}= & \frac{1}{16} \sum_{m, n}\left(\mathcal{F}^{G_{3,1}}\right)_{m, n} 2^{m+n} \\
& \times\left(\prod_{i=1}^{m} \prod_{j=1}^{n} \partial_{\mu_{i}} \partial_{\nu_{j}}\left(F_{a b}^{1} F_{p q}^{1}\right) F_{b a}^{2} F_{q p}^{2} \partial^{\mu_{i}}\left(F_{c d}^{3} F_{r s}^{3}\right) \partial^{\nu_{j}}\left(F_{d c}^{4} F_{s r}^{4}\right)\right) .
\end{aligned}
$$

plus permutations. 
- The S-matrix corresponding to $G_{\mathbf{3}, 2}$ in (6.17) is specified by the momenta polynomial $\mathcal{F}^{G_{3,2}}(s, u)$ which has the $\mathbb{Z}_{2}$ symmetry $(s \leftrightarrow u)$. Explicitly it is given by

$$
\begin{aligned}
& S^{G_{3,2}}=\frac{1}{4}\left(\mathcal{F}^{G_{3,2}}(s, u)\right. {\left[\left(p_{p}^{1} \epsilon_{q}^{1}-p_{q}^{1} \epsilon_{p}^{1}\right)\left(p_{p}^{2} \epsilon_{q}^{2}-p_{q}^{2} \epsilon_{p}^{2}\right)\left(p_{v}^{3} \epsilon_{w}^{3}-p_{w}^{3} \epsilon_{v}^{3}\right)\left(p_{v}^{4} \epsilon_{w}^{4}-p_{w}^{4} \epsilon_{v}^{4}\right)\right.} \\
&\left.\times\left(p_{r}^{1} \epsilon_{s}^{1}-p_{s}^{1} \epsilon_{r}^{1}\right)\left(p_{r}^{4} \epsilon_{s}^{4}-p_{s}^{4} \epsilon_{r}^{4}\right)\left(p_{t}^{2} \epsilon_{u}^{2}-p_{u}^{2} \epsilon_{t}^{2}\right)\left(p_{t}^{3} \epsilon_{u}^{3}-p_{u}^{3} \epsilon_{t}^{3}\right)\right] \\
&\left.+\mathcal{F}^{G_{3}, 2}(t, u)[3 \leftrightarrow 2]+\mathcal{F}^{G_{3,2}}(s, t)[3 \leftrightarrow 4]\right) .
\end{aligned}
$$

The most general descendant is

$$
L^{G_{3,2}}=\sum_{m, n}\left(\mathcal{F}^{G_{3,2}}\right)_{m, n} 2^{m+n}\left(\prod_{i=1}^{m} \prod_{j=1}^{n}\left(\partial_{\mu_{i}} \partial_{\nu_{j}} R_{p q r s}\right)\left(\partial^{\mu_{i}} R_{p q t u}\right) R_{t u v w}\left(\partial^{\nu_{j}} R_{r s v w}\right)\right) .
$$

That the descendant Lagrangian (K.7) generates the S-matrix (K.6) is easy to see; the Lagrangian

$$
R_{\text {pqrs }} R_{\text {pqtu }} R_{\text {tuvw }} R_{\text {rsvw }}
$$

linearizes to give $\operatorname{Tr}\left(F^{1} F^{2}\right) \operatorname{Tr}\left(F^{3} F^{4}\right) \operatorname{Tr}\left(F^{1} F^{4}\right) \operatorname{Tr}\left(F^{2} F^{3}\right)$ plus permutations. This structure has an obvious extra $\mathbb{Z}_{2}$ symmetry of 1 to 3 exchange. The descendant Lagrangian (K.7) then linearizes to give,

$$
\frac{1}{16} \sum_{m, n}\left(\mathcal{F}^{G_{3,2}}\right)_{m, n} 2^{m+n}\left(\prod_{i=1}^{m} \prod_{j=1}^{n} \partial_{\mu_{i}} \partial_{\nu_{j}}\left(F_{p q}^{1} F_{r s}^{1}\right) \partial^{\mu_{i}}\left(F_{p q}^{2} F_{t u}^{2}\right) F_{t u}^{3} F_{v w}^{3} \partial^{\nu_{j}}\left(F_{r s}^{4} F_{v w}^{4}\right)\right) \text {. }
$$

plus permutations.

- The most general S-matrix corresponding $G_{\mathbf{3}, 3}$ in (6.17) is specified by the momenta polynomials $\mathcal{F}^{G_{3,3}}(s, u)$ which is symmetric under $(s \leftrightarrow u)$.

$$
\begin{aligned}
S^{G_{3,3}}=\frac{1}{4}\left(\mathcal { F } ^ { G _ { 3 , 3 } } ( s , u ) \left[\left(p_{p}^{1} \epsilon_{q}^{1}-p_{q}^{1} \epsilon_{p}^{1}\right)\left(p_{p}^{2} \epsilon_{t}^{2}-p_{t}^{2} \epsilon_{p}^{2}\right)\left(p_{t}^{3} \epsilon_{v}^{3}-p_{v}^{3} \epsilon_{t}^{3}\right)\left(p_{q}^{4} \epsilon_{v}^{4}-p_{v}^{4} \epsilon_{q}^{4}\right)\right.\right. \\
\left.\quad \times\left(p_{r}^{1} \epsilon_{s}^{1}-p_{s}^{1} \epsilon_{r}^{1}\right)\left(p_{r}^{2} \epsilon_{u}^{2}-p_{u}^{2} \epsilon_{r}^{2}\right)\left(p_{u}^{3} \epsilon_{w}^{3}-p_{w}^{3} \epsilon_{u}^{3}\right)\left(p_{s}^{4} \epsilon_{w}^{4}-p_{w}^{4} \epsilon_{s}^{4}\right)\right] \\
\left.+\mathcal{F}^{G_{3,3}}(t, u)[3 \leftrightarrow 2]+\mathcal{F}^{G_{3,3}}(s, t)[3 \leftrightarrow 4]\right) .
\end{aligned}
$$

The most general descendant Lagrangian that gives rise to the S-matrix (K.10) is as follows

$$
L^{G_{3,3}}=\sum_{m, n}\left(\mathcal{F}^{G_{3,3}}\right)_{m, n} 2^{m+n}\left(\prod_{i=1}^{m} \prod_{j=1}^{n}\left(\partial_{\nu_{j}} \partial_{\mu_{i}} R_{p q r s}\right)\left(\partial^{\mu_{i}} R_{p t r u}\right) R_{t v u w}\left(\partial^{\nu_{j}} R_{q v s w}\right)\right) .
$$

In order to see the fact that Lagrangian (K.11) results in the S-matrix (K.10), we note that

$$
R_{p q r s} R_{p t r u} R_{t v u w} R_{q v s w}
$$


linearizes to $\operatorname{Tr}\left(F^{1} F^{2} F^{3} F^{4}\right) \operatorname{Tr}\left(F^{1} F^{2} F^{3} F^{4}\right)$ plus permutations. This structure, like $\operatorname{Tr}\left(F^{4}\right)$ again has $\mathbb{Z}_{2}$ symmetry of $1 \leftrightarrow 3$, which manifests in the $u \leftrightarrow s$ symmetry of the momenta functions $\mathcal{F}^{G_{3,3}}(s, u)$. It follows therefore the Lagrangian (K.11) linearizes to

$$
\frac{1}{16} \sum_{m, n}\left(\mathcal{F}^{G_{3}, 3}\right)_{m . n} 2^{m+n}\left(\prod_{i=1}^{m} \prod_{j=1}^{n} \partial_{\nu_{j}} \partial_{\mu_{i}}\left(F_{p q}^{1} F_{r s}^{1}\right) \partial^{\mu_{i}}\left(F_{p t}^{2} F_{r u}^{2}\right) F_{t v}^{3} F_{u w}^{3} \partial^{\nu_{j}}\left(F_{q v}^{4} F_{s w}^{4}\right)\right)
$$

plus permutations.

- The S-matrix corresponding to $G_{\mathbf{3}, 4}$ in (6.17) is specified by the momenta polynomials $\mathcal{F}^{G_{3,4}}(s, t)$ with the $\mathbb{Z}_{2}$ symmetry $(s \leftrightarrow t)$. The explicit S-matrix is as follows

$$
\begin{aligned}
S^{G_{\mathbf{3}, 4}}=\frac{1}{4}\left(\mathcal { F } ^ { G _ { \mathbf { 3 } , 4 } } ( s , t ) \left[\left(p_{p}^{1} \epsilon_{q}^{1}-p_{q}^{1} \epsilon_{p}^{1}\right)\left(p_{p}^{2} \epsilon_{t}^{2}-p_{t}^{2} \epsilon_{p}^{2}\right)\left(p_{t}^{3} \epsilon_{v}^{3}-p_{v}^{3} \epsilon_{t}^{3}\right)\left(p_{q}^{4} \epsilon_{v}^{4}-p_{v}^{4} \epsilon_{q}^{4}\right)\right.\right. \\
\left.\quad \times\left(p_{r}^{1} \epsilon_{s}^{1}-p_{s}^{1} \epsilon_{r}^{1}\right)\left(p_{u}^{2} \epsilon_{w}^{2}-p_{w}^{2} \epsilon_{u}^{2}\right)\left(p_{w}^{3} \epsilon_{s}^{3}-p_{s}^{3} \epsilon_{w}^{3}\right)\left(p_{r}^{4} \epsilon_{u}^{4}-p_{u}^{4} \epsilon_{r}^{4}\right)\right] \\
\left.+\mathcal{F}^{G_{\mathbf{3}, 4}}(s, u)[3 \leftrightarrow 4]+\mathcal{F}^{G_{3,4}}(u, t)[2 \leftrightarrow 4]\right) .
\end{aligned}
$$

The most general descendant which gives rise to this S-matrix is given by

$$
L^{G_{\mathbf{3}, 4}}=\sum_{m, n}\left(\mathcal{F}^{G_{\mathbf{3}, 4}}\right)_{m, n} 2^{m+n}\left(\prod_{i=1}^{m} \prod_{j=1}^{n}\left(\partial_{\nu_{j}} \partial_{\mu_{i}} R_{p q r s}\right)\left(\partial^{\mu_{i}} R_{p t u w}\right)\left(\partial^{\nu_{j}} R_{t v w s}\right) R_{q v r u}\right) .
$$

In order to see the fact that Lagrangian (K.15) results in the S-matrix (K.14), we note that

$$
R_{\text {pqrs }} R_{\text {ptuw }} R_{\text {tvws }} R_{\text {qvru }}
$$

linearizes to $\operatorname{Tr}\left(F^{1} F^{2} F^{3} F^{4}\right) \operatorname{Tr}\left(F^{1} F^{3} F^{2} F^{4}\right)$ plus permutations. This structure has $\mathbb{Z}_{2}$ symmetry of $2 \leftrightarrow 3$ (and hence the $\mathbb{Z}_{2}$ symmetry of the momenta polynomials $\left.\mathcal{F}^{G_{3,4}}(s, t)\right)$. When linearized, the most general descendant Lagrangian (K.15) becomes,

$$
\frac{1}{16} \sum_{m, n}\left(\mathcal{F}^{G_{3}, 4}\right)_{m, n} 2^{m+n}\left(\prod_{i=1}^{m} \prod_{j=1}^{n} \partial_{\nu_{j}} \partial_{\mu_{i}}\left(F_{p q}^{1} F_{r s}^{1}\right) \partial^{\mu_{i}}\left(F_{p t}^{2} F_{u w}^{2}\right) \partial^{\nu_{j}}\left(F_{t v}^{3} F_{w s}^{3}\right) F_{q v}^{4} F_{r u}^{4}\right) .
$$

plus permutations.

- The S-matrix corresponding to $G_{\mathbf{3}, 5}$ in (6.17) is specified by momenta polynomials $\mathcal{F}^{G_{3,5}}(t, u)$ which has a $\mathbb{Z}_{2}$ symmetry in $(t \leftrightarrow u)$.

$$
\begin{aligned}
& S^{G_{3}, 5}=\frac{1}{4}\left(\mathcal{F}^{G_{3,5}}(t, u)\right. {\left[\left(p_{p}^{1} \epsilon_{q}^{1}-p_{q}^{1} \epsilon_{p}^{1}\right)\left(p_{p}^{2} \epsilon_{q}^{2}-p_{q}^{2} \epsilon_{p}^{2}\right)\left(p_{v}^{3} \epsilon_{w}^{3}-p_{w}^{3} \epsilon_{v}^{3}\right)\left(p_{v}^{4} \epsilon_{w}^{4}-p_{w}^{4} \epsilon_{v}^{4}\right)\right.} \\
&\left.\times\left(p_{r}^{1} \epsilon_{s}^{1}-p_{s}^{1} \epsilon_{r}^{1}\right)\left(p_{t}^{2} \epsilon_{u}^{2}-p_{u}^{2} \epsilon_{t}^{2}\right)\left(p_{r}^{3} \epsilon_{t}^{3}-p_{t}^{3} \epsilon_{r}^{3}\right)\left(p_{s}^{4} \epsilon_{u}^{4}-p_{u}^{4} \epsilon_{s}^{4}\right)\right] \\
&\left.+\mathcal{F}^{G_{3,5}}(u, s)[2 \leftrightarrow 3]+\mathcal{F}^{G_{3}, 5}(s, t)[2 \leftrightarrow 4]\right)
\end{aligned}
$$


The S-matrix (K.18) is produced by the general descendant Lagrangian

$$
L^{G_{3,5}}=\sum_{m, n}\left(\mathcal{F}^{G_{3,5}}\right)_{m, n} 2^{m+n}\left(\prod_{i=1}^{m} \prod_{j=1}^{n}\left(\partial_{\nu_{j}} \partial_{\mu_{i}} R_{p q r s}\right) R_{p q t u}\left(\partial^{\mu_{i}} R_{r t v w}\right)\left(\partial^{\nu_{i}} R_{\text {suvw }}\right)\right) .
$$

In order to see the fact that Lagrangian (K.19) results in the S-matrix (K.18), we note that

$$
R_{\text {pqrs }} R_{\text {pqtu }} R_{\text {rtvw }} R_{\text {suvw }}
$$

linearizes to $\operatorname{Tr}\left(F^{1} F^{2}\right) \operatorname{Tr}\left(F^{3} F^{4}\right) \operatorname{Tr}\left(F^{1} F^{3} F^{2} F^{4}\right)$ plus permutation. The general descendant Lagrangian (K.19) therefore linearizes to

$$
\frac{1}{16} \sum_{m, n}\left(\mathcal{F}^{G_{3,5}}\right)_{m, n} 2^{m+n}\left(\prod_{i=1}^{m} \prod_{j=1}^{n} \partial_{\nu_{j}} \partial_{\mu_{i}}\left(F_{p q}^{1} F_{r s}^{1}\right) F_{p q}^{2} F_{t u}^{2} \partial^{\mu_{i}}\left(F_{r t}^{3} F_{v w}^{3}\right) \partial^{\nu_{i}}\left(F_{s u}^{4} F_{v w}^{4}\right)\right)
$$

plus permutations.

- The most general S-matrix generated by $G_{\mathbf{6}}=G_{\mathbf{3}, 6} \oplus G_{\mathbf{3}_{\mathrm{A}}}$ is specified by an arbitrary polynomial $\mathcal{F}^{G_{6}}(t, u)$ with no symmetry restrictions. The corresponding S-matrix is

$$
\begin{aligned}
S^{G_{6}}= & \frac{1}{4} \mathcal{F}^{G_{6}}(s, t)\left[\left(p_{p}^{1} \epsilon_{q}^{1}-p_{q}^{1} \epsilon_{p}^{1}\right)\left(p_{p}^{2} \epsilon_{q}^{2}-p_{q}^{2} \epsilon_{p}^{2}\right)\left(p_{u}^{3} \epsilon_{v}^{3}-p_{v}^{3} \epsilon_{u}^{3}\right)\left(p_{u}^{4} \epsilon_{v}^{4}-p_{v}^{4} \epsilon_{u}^{4}\right)\right. \\
& \left.\times\left(p_{r}^{1} \epsilon_{s}^{1}-p_{s}^{1} \epsilon_{r}^{1}\right)\left(p_{r}^{2} \epsilon_{t}^{2}-p_{t}^{2} \epsilon_{r}^{2}\right)\left(p_{w}^{3} \epsilon_{t}^{3}-p_{t}^{3} \epsilon_{w}^{3}\right)\left(p_{w}^{4} \epsilon_{s}^{4}-p_{s}^{4} \epsilon_{w}^{4}\right)\right] \\
& \left.+S_{3} \text { permutations (also act on } s, t, u\right)
\end{aligned}
$$

This S-matrix (K.22) is produced (up to proportionality) by the Lagrangian

$$
L^{G_{\mathbf{6}}}=\sum_{m, n}\left(\mathcal{F}^{G_{\mathbf{6}}}\right)_{m . n} 2^{m+n}\left(\prod_{i=1}^{m} \prod_{j=1}^{n}\left(\partial_{\mu_{i}} \partial_{\nu_{j}} R_{p q r s}\right)\left(\partial^{\mu_{i}} R_{p q r t}\right)\left(\partial^{\nu_{i}} R_{u v w t}\right) R_{u v w s}\right)
$$

The fact that (K.23) yields the S-matrix (K.22) follows from the fact that

$$
R_{\text {pqrs }} R_{\text {pqrt }} R_{\text {uvwt }} R_{\text {uvws }}
$$

linearizes to $\operatorname{Tr}\left(F^{1} F^{2}\right) \operatorname{Tr}\left(F^{3} F^{4}\right) \operatorname{Tr}\left(F^{1} F^{2} F^{3} F^{4}\right)$ plus permutations (where the superscript, as usual, labels particles). It follows that the Lagrangian (K.23) linearizes to

$$
\frac{1}{16} \sum_{m, n}\left(\mathcal{F}^{G_{\mathbf{6}}}\right)_{m . n} 2^{m+n}\left(\prod_{i=1}^{m} \prod_{j=1}^{n} \partial_{\mu_{i}} \partial_{\nu_{j}}\left(F_{p q}^{1} F_{r s}^{1}\right) \partial^{\mu_{i}}\left(F_{p q}^{2} F_{r t}^{2}\right) \partial^{\nu_{i}}\left(F_{u v}^{3} F_{w t}^{3}\right) F_{u v}^{4} F_{w s}^{4}\right)
$$

plus permutations. The replacement rule $\partial_{\mu} \rightarrow i k_{\mu}$ then turns (K.25) into (K.22). 
- The S-matrix corresponding to $G_{\mathbf{3}, 7}$ in (6.17) is specified by $\mathcal{F}^{G_{3,7}}(t, u)$ which has $\mathbb{Z}_{2}$ symmetry of $(t \leftrightarrow u)$. Explicitly,

$$
\begin{aligned}
S^{G_{3,7}}= & \frac{1}{16}\left(\mathcal{F}^{G_{3,7}}(t, u)\left(p_{p}^{1} \epsilon_{q}^{1}-p_{q}^{1} \epsilon_{p}^{1}\right)\left(p_{p}^{2} \epsilon_{q}^{2}-p_{q}^{2} \epsilon_{p}^{2}\right)\left(p_{r}^{3} \epsilon_{s}^{3}-p_{s}^{3} \epsilon_{r}^{3}\right)\left(p_{r}^{4} \epsilon_{s}^{4}-p_{s}^{4} \epsilon_{r}^{4}\right)\right. \\
& +\mathcal{F}^{G_{3,7}}(s, u)\left(p_{p}^{1} \epsilon_{q}^{1}-p_{q}^{1} \epsilon_{p}^{1}\right)\left(p_{p}^{3} \epsilon_{q}^{3}-p_{q}^{3} \epsilon_{p}^{3}\right)\left(p_{r}^{2} \epsilon_{s}^{2}-p_{s}^{2} \epsilon_{r}^{2}\right)\left(p_{r}^{4} \epsilon_{s}^{4}-p_{s}^{4} \epsilon_{r}^{4}\right) \\
& \left.+\mathcal{F}^{G_{3}, 7}(t, s)\left(p_{p}^{1} \epsilon_{q}^{1}-p_{q}^{1} \epsilon_{p}^{1}\right)\left(p_{p}^{4} \epsilon_{q}^{4}-p_{q}^{4} \epsilon_{p}^{4}\right)\left(p_{r}^{3} \epsilon_{s}^{3}-p_{s}^{3} \epsilon_{r}^{3}\right)\left(p_{r}^{2} \epsilon_{s}^{2}-p_{s}^{2} \epsilon_{r}^{2}\right)\right) \\
& \times\left(\left(p_{a}^{1} \epsilon_{b}^{1}-p_{b}^{1} \epsilon_{a}^{1}\right) p_{a}^{2}\left(p_{\mu}^{2} \epsilon_{\nu}^{2}-p_{\nu}^{2} \epsilon_{\mu}^{2}\right) p_{b}^{3}\left(p_{\nu}^{3} \epsilon_{\alpha}^{3}-p_{\alpha}^{3} \epsilon_{\nu}^{3}\right)\left(p_{\alpha}^{4} \epsilon_{\mu}^{4}-p_{\mu}^{4} \epsilon_{\alpha}^{4}\right)\right. \\
& +\left(p_{a}^{2} \epsilon_{b}^{2}-p_{b}^{2} \epsilon_{a}^{2}\right) p_{a}^{1}\left(p_{\mu}^{1} \epsilon_{\nu}^{1}-p_{\nu}^{1} \epsilon_{\mu}^{1}\right) p_{b}^{4}\left(p_{\nu}^{4} \epsilon_{\alpha}^{4}-p_{\alpha}^{4} \epsilon_{\nu}^{4}\right)\left(p_{\alpha}^{3} \epsilon_{\mu}^{3}-p_{\mu}^{3} \epsilon_{\alpha}^{3}\right) \\
& +\left(p_{a}^{3} \epsilon_{b}^{3}-p_{b}^{3} \epsilon_{a}^{3}\right) p_{a}^{4}\left(p_{\mu}^{4} \epsilon_{\nu}^{4}-p_{\nu}^{4} \epsilon_{\mu}^{4}\right) p_{b}^{1}\left(p_{\nu}^{1} \epsilon_{\alpha}^{1}-p_{\alpha}^{1} \epsilon_{\nu}^{1}\right)\left(p_{\alpha}^{2} \epsilon_{\mu}^{2}-p_{\mu}^{2} \epsilon_{\alpha}^{2}\right) \\
& \left.+\left(p_{a}^{4} \epsilon_{b}^{4}-p_{b}^{4} \epsilon_{a}^{4}\right) p_{a}^{3}\left(p_{\mu}^{3} \epsilon_{\nu}^{3}-p_{\nu}^{3} \epsilon_{\mu}^{3}\right) p_{b}^{2}\left(p_{\nu}^{2} \epsilon_{\alpha}^{2}-p_{\alpha}^{2} \epsilon_{\nu}^{2}\right)\left(p_{\alpha}^{1} \epsilon_{\mu}^{1}-p_{\mu}^{1} \epsilon_{\alpha}^{1}\right)\right)
\end{aligned}
$$

The S-matrix (K.26) is generated by the descendant Lagrangian

$$
\begin{aligned}
L^{G_{3,7}}= & -\sum_{m, n}\left(\mathcal{F}^{G_{3,7}}\right)_{m, n} 2^{m+n} \\
& \times\left(\prod_{i=1}^{m} \prod_{j=1}^{n}\left(\partial_{\mu_{i}} \partial_{\nu_{j}} R_{p q a b}\right)\left(\partial_{a} R_{q p \mu \nu}\right)\left(\partial_{b} \partial^{\mu_{i}} R_{r s \nu \alpha}\right)\left(\partial^{\nu_{j}} R_{s r \alpha \mu}\right)\right)
\end{aligned}
$$

In order to see that S-matrix (K.26) is generated by (K.27), we note that

$$
R_{p q a b} \partial_{a} R_{q p \mu \nu} \partial_{b} R_{r s \nu \alpha} R_{s r \alpha \mu}
$$

linearizes to give $\operatorname{Tr}\left(F^{1} F^{2}\right) \operatorname{Tr}\left(F^{3} F^{4}\right) F_{a b}^{1} \operatorname{Tr}\left(p_{a}^{2} F^{2} p_{b}^{3} F^{3} F^{4}\right)$ plus permutations. This structure again has only $\mathbb{Z}_{2}$ symmetry of $3 \leftrightarrow 4$. The descendant Lagrangian (K.27) then linearizes to give

$$
\begin{aligned}
& -\frac{1}{16} \sum_{m, n}\left(\mathcal{F}^{G_{3,7}}\right)_{m, n} 2^{m+n} \\
& \quad \times\left(\prod_{i=1}^{m} \prod_{j=1}^{n} \partial_{\mu_{i}} \partial_{\nu_{j}}\left(F_{p q}^{1} F_{a b}^{1}\right) \partial_{a} F_{q p}^{2} F_{\mu \nu}^{2} \partial_{b} \partial^{\mu_{i}}\left(F_{r s}^{3} F_{\nu \alpha}^{3}\right) \partial^{\nu_{j}}\left(F_{s r}^{4} F_{\alpha \mu}^{4}\right)\right)
\end{aligned}
$$

plus permutations.

- The S-matrix corresponding to $G_{\mathbf{3}, 8}$ in (6.17) is specified by the momenta functions $\mathcal{F}^{G_{3,8}}(s, u)$ which has the $\mathbb{Z}_{2}$ symmetry $(s \leftrightarrow u)$. In equations,

$$
\begin{aligned}
S^{G_{3,8}}= & \frac{1}{16}\left(\mathcal{F}^{G_{3,8}}(s, u)\left(p_{p}^{1} \epsilon_{q}^{1}-p_{q}^{1} \epsilon_{p}^{1}\right)\left(p_{q}^{2} \epsilon_{r}^{2}-p_{r}^{2} \epsilon_{q}^{2}\right)\left(p_{r}^{3} \epsilon_{s}^{3}-p_{s}^{3} \epsilon_{r}^{3}\right)\left(p_{s}^{4} \epsilon_{p}^{4}-p_{p}^{4} \epsilon_{s}^{4}\right)\right. \\
& \times \mathcal{F}^{G_{3,8}}(t, u)\left(p_{p}^{1} \epsilon_{q}^{1}-p_{q}^{1} \epsilon_{p}^{1}\right)\left(p_{q}^{3} \epsilon_{r}^{3}-p_{r}^{3} \epsilon_{q}^{3}\right)\left(p_{r}^{2} \epsilon_{s}^{2}-p_{s}^{2} \epsilon_{r}^{2}\right)\left(p_{s}^{4} \epsilon_{p}^{4}-p_{p}^{4} \epsilon_{s}^{4}\right) \\
& \left.+\mathcal{F}^{G_{3,8}}(t, s)\left(p_{p}^{1} \epsilon_{q}^{1}-p_{q}^{1} \epsilon_{p}^{1}\right)\left(p_{q}^{3} \epsilon_{r}^{3}-p_{r}^{3} \epsilon_{q}^{3}\right)\left(p_{r}^{4} \epsilon_{s}^{4}-p_{s}^{4} \epsilon_{r}^{4}\right)\left(p_{s}^{2} \epsilon_{p}^{2}-p_{p}^{2} \epsilon_{s}^{2}\right)\right) \\
& \times\left(\left(p_{a}^{1} \epsilon_{b}^{1}-p_{b}^{1} \epsilon_{a}^{1}\right) p_{a}^{2}\left(p_{\mu}^{2} \epsilon_{\nu}^{2}-p_{\nu}^{2} \epsilon_{\mu}^{2}\right) p_{b}^{3}\left(p_{\nu}^{3} \epsilon_{\alpha}^{3}-p_{\alpha}^{3} \epsilon_{\nu}^{3}\right)\left(p_{\alpha}^{4} \epsilon_{\mu}^{4}-p_{\mu}^{4} \epsilon_{\alpha}^{4}\right)\right. \\
& +\left(p_{a}^{2} \epsilon_{b}^{2}-p_{b}^{2} \epsilon_{a}^{2}\right) p_{a}^{1}\left(p_{\mu}^{1} \epsilon_{\nu}^{1}-p_{\nu}^{1} \epsilon_{\mu}^{1}\right) p_{b}^{4}\left(p_{\nu}^{4} \epsilon_{\alpha}^{4}-p_{\alpha}^{4} \epsilon_{\nu}^{4}\right)\left(p_{\alpha}^{3} \epsilon_{\mu}^{3}-p_{\mu}^{3} \epsilon_{\alpha}^{3}\right) \\
& +\left(p_{a}^{3} \epsilon_{b}^{3}-p_{b}^{3} \epsilon_{a}^{3}\right) p_{a}^{4}\left(p_{\mu}^{4} \epsilon_{\nu}^{4}-p_{\nu}^{4} \epsilon_{\mu}^{4}\right) p_{b}^{1}\left(p_{\nu}^{1} \epsilon_{\alpha}^{1}-p_{\alpha}^{1} \epsilon_{\nu}^{1}\right)\left(p_{\alpha}^{2} \epsilon_{\mu}^{2}-p_{\mu}^{2} \epsilon_{\alpha}^{2}\right) \\
& \left.+\left(p_{a}^{4} \epsilon_{b}^{4}-p_{b}^{4} \epsilon_{a}^{4}\right) p_{a}^{3}\left(p_{\mu}^{3} \epsilon_{\nu}^{3}-p_{\nu}^{3} \epsilon_{\mu}^{3}\right) p_{b}^{2}\left(p_{\nu}^{2} \epsilon_{\alpha}^{2}-p_{\alpha}^{2} \epsilon_{\nu}^{2}\right)\left(p_{\alpha}^{1} \epsilon_{\mu}^{1}-p_{\mu}^{1} \epsilon_{\alpha}^{1}\right)\right)
\end{aligned}
$$


The descendant is of the general form

$$
\begin{aligned}
L^{G_{3,8}}= & -\sum_{m, n}\left(\mathcal{F}^{G_{3,8}}\right)_{m, n} 2^{m+n} \\
& \times\left(\prod_{i=1}^{m} \prod_{j=1}^{n}\left(\partial_{\mu_{i}} \partial_{\nu_{j}} R_{p q a b}\right)\left(\partial_{a} \partial^{\mu_{i}} R_{q r \mu \nu}\right)\left(\partial_{b} R_{r s \nu \alpha}\right)\left(\partial^{\nu_{j}} R_{s p \alpha \mu}\right)\right)
\end{aligned}
$$

Reader can convince himself/herself that the descendant Lagrangian (K.31) gives rise to the S-matrix (K.30) by noting that

$$
R_{p q a b} \partial_{a} R_{q r \mu \nu} \partial_{b} R_{r s \nu \alpha} R_{s p \alpha \mu}
$$

linearizes to give $\operatorname{Tr}\left(F^{1} F^{2} F^{3} F^{4}\right) F_{a b}^{1} \operatorname{Tr}\left(p_{a}^{2} F^{2} p_{b}^{3} F^{3} F^{4}\right)$ plus permutations. This structure has neither $\mathbb{Z}_{2} \times \mathbb{Z}_{2}$, which although is preserved by the first trace but broken by the $F \operatorname{Tr}(\ldots)$ part, nor it has $S^{3}$ which is preserved by the $F \operatorname{Tr}(\ldots)$ part but broken by the $\operatorname{Tr}\left(F^{4}\right)$ part. Only $\mathbb{Z}_{2}$ is preserved, that is just $2 \leftrightarrow 4$ flip symmetry. Consequently the $\mathbb{Z}_{2} \times \mathbb{Z}_{2}$ symmetrization had to be done explicitly in (K.30). The descendant Lagrangian (K.31) then linearizes to

$$
\begin{aligned}
& -\frac{1}{16} \sum_{m, n}\left(\mathcal{F}^{G_{3,8}}\right)_{m, n} 2^{m+n} \\
& \times\left.\left(\prod_{i=1}^{m} \prod_{j=1}^{n} \partial_{\mu_{i}} \partial_{\nu_{j}}\left(F_{p q}^{1} F_{a b}^{1}\right) \partial_{a} \partial^{\mu_{i}}\left(F_{q r}^{2} F_{\mu \nu}^{2}\right) \partial_{b}\left(F_{r s}^{3} F_{\nu \alpha}^{3}\right) \partial^{\nu_{j}}\left(F_{s p}^{4} F_{\alpha \mu}^{4}\right)\right)\right|_{\mathbb{Z}_{2} \times \mathbb{Z}_{2}}
\end{aligned}
$$

- The S-matrix corresponding to $G_{\mathbf{S}, 2}$ in (6.17) is given by the momenta polynomial $\mathcal{F}^{G_{\mathbf{S}, 2}}(s, t)$ which is fully symmetric in $s, t$ and $u$. The explicit expression for the $\mathrm{S}$-matrix is given by,

$$
\begin{aligned}
S^{G_{\mathbf{S}, 2}=} & \frac{1}{16}\left(\mathcal{F}^{G_{\mathbf{S}, 2}}(s, t)\right) \\
& \times\left[\left(p_{a}^{1} \epsilon_{b}^{1}-p_{b}^{1} \epsilon_{a}^{1}\right) p_{a}^{2}\left(p_{\mu}^{2} \epsilon_{\nu}^{2}-p_{\nu}^{2} \epsilon_{\mu}^{2}\right) p_{b}^{3}\left(p_{\nu}^{3} \epsilon_{\alpha}^{3}-p_{\alpha}^{3} \epsilon_{\nu}^{3}\right)\left(p_{\alpha}^{4} \epsilon_{\mu}^{4}-p_{\mu}^{4} \epsilon_{\alpha}^{4}\right)\right. \\
& \times\left(p_{p}^{1} \epsilon_{q}^{1}-p_{q}^{1} \epsilon_{p}^{1}\right) p_{p}^{2}\left(p_{\beta}^{2} \epsilon_{\gamma}^{2}-p_{\gamma}^{2} \epsilon_{\beta}^{2}\right) p_{q}^{3}\left(p_{\gamma}^{3} \epsilon_{\delta}^{3}-p_{\delta}^{3} \epsilon_{\gamma}^{3}\right)\left(p_{\delta}^{4} \epsilon_{\beta}^{4}-p_{\beta}^{4} \epsilon_{\delta}^{4}\right) \\
& +(1 \leftrightarrow 2)+(1 \leftrightarrow 3)+(1 \leftrightarrow 4)]
\end{aligned}
$$

The most general descendant Lagrangian giving rise to (K.34) is

$$
\begin{aligned}
L^{G_{\mathbf{S}, 2}}= & \sum_{m, n}\left(\mathcal{F}^{G_{\mathbf{S}, 2}}\right)_{m, n} 2^{m+n} \\
& \times\left(\prod_{i=1}^{m} \prod_{j=1}^{n}\left(\partial_{\mu_{i}} \partial_{\nu_{j}} R_{a b p q}\right)\left(\partial^{\mu_{i}} \partial_{p} \partial_{a} R_{\mu \nu \beta \gamma}\right)\left(\partial^{\nu_{j}} \partial_{q} \partial_{b} R_{\nu \alpha \gamma \delta}\right) R_{\alpha \mu \delta \beta}\right)
\end{aligned}
$$

It is easy to see that the descendant Lagrangian (K.35) generates the S-matrix (K.34). Consider the Lagrangian

$$
R_{a b p q} \partial_{p} \partial_{a} R_{\mu \nu \beta \gamma} \partial_{q} \partial_{b} R_{\nu \alpha \gamma \delta} R_{\alpha \mu \delta \beta}
$$


which linearizes to give $F_{p q}^{1} \operatorname{Tr}\left(p_{p}^{2} F^{2} p_{q}^{3} F^{3} F^{4}\right) F_{a b}^{1} \operatorname{Tr}\left(p_{a}^{2} F^{2} p_{b}^{3} F^{3} F^{4}\right)$ plus permutations. This structure has $S^{3}$ symmetry, because $2,3,4$ can be permuted and the structure remains invariant. The descendant Lagrangian (K.35) linearizes to give,

$$
\begin{aligned}
& \frac{1}{16} \sum_{m, n}\left(\mathcal{F}^{G_{\mathbf{S}, 2}}\right)_{m, n} 2^{m+n} \\
& \quad \times\left(\prod_{i=1}^{m} \prod_{j=1}^{n} \partial_{\mu_{i}} \partial_{\nu_{j}}\left(F_{a b}^{1} F_{p q}^{1}\right) \partial^{\mu_{i}} \partial_{p} \partial_{a}\left(F_{\mu \nu}^{2} F_{\beta \gamma}^{2}\right) \partial^{\nu_{j}} \partial_{q} \partial_{b}\left(F_{\nu \alpha}^{3} F_{\gamma \delta}^{3}\right) F_{\alpha \mu}^{4} F_{\delta \beta}^{4}\right)
\end{aligned}
$$

plus permutations.

- Finally we turn to the specification of the S-matrices descended from $G_{\mathbf{S}, 1}$. If we are interested in specifying only the S-matrix - and not the Lagrangian that gives rise to this S-matrix - this job is easily done. In addition to the S-matrices already listed in this appendix we have one additional contribution specified by $\mathcal{F}^{G, 1}$, a fully symmetric polynomial of $s, t, u$ that is otherwise unconstrained. The S-matrix is given by

$$
S_{D \geq 7}^{G_{\mathbf{S}, 1}}=24\left(3 \mathcal{F}^{G \mathbf{S}, 1}(t, u) \epsilon^{i j k l m n p} \epsilon^{a s d f g h j} \epsilon_{i}^{1} \epsilon_{j}^{2} \epsilon_{k}^{3} \epsilon_{l}^{4} p_{m}^{1} p_{n}^{2} p_{p}^{3} \epsilon_{a}^{1} \epsilon_{s}^{2} \epsilon_{d}^{3} \epsilon_{f}^{4} p_{g}^{1} p_{h}^{2} p_{j}^{3}\right)
$$

At the level of S-matrices we have now completed our listings. The most general sum of (K.38), (K.22), (K.18), (K.10), (K.14), (K.1), (K.6), (K.30), (K.26), (K.34) gives the most general local S-matrix for gravitational scattering in $D \geq 8$.

- If, on the other hand, we are also interested in listing the Lagrangians that give rise to the S-matrices that descend from $G_{\mathbf{S}, 1}$ we are forced to proceed differently. In this case we do not include (K.38) in our listing of S-matrices but instead replace (K.38) by the most general S-matrix corresponding to $G_{\mathbf{3}, 9}$ (6.1). Such an S-matrix is given by the momenta polynomial $\mathcal{F}^{G, 9}(t, u)$ which is symmetric in $s \leftrightarrow u$. As the $\mathbf{1}_{S}$ part of $G_{\mathbf{3}, 9}$ can be eliminated using (6.2), only the $\mathbf{2}_{M}$ part suffices for us. This means we further impose,

$$
\mathcal{F}^{G_{3,9}}(t, u)+\mathcal{F}^{G_{3,9}}(u, s)+\mathcal{F}^{G_{3,9}}(s, t)=0 .
$$

The explicit expression for the S-matrix is given by,

$$
\begin{aligned}
& S^{G_{3,9}}=\frac{1}{4}\left(\mathcal { F } ^ { G _ { 3 , 9 } } ( t , u ) \left[\left(p_{p}^{1} \epsilon_{q}^{1}-p_{q}^{1} \epsilon_{p}^{1}\right)\left(p_{p}^{2} \epsilon_{t}^{2}-p_{t}^{2} \epsilon_{p}^{2}\right)\left(p_{t}^{3} \epsilon_{v}^{3}-p_{v}^{3} \epsilon_{t}^{3}\right)\left(p_{u}^{4} \epsilon_{v}^{4}-p_{v}^{4} \epsilon_{u}^{4}\right)\right.\right. \\
& \left.\times\left(p_{r}^{1} \epsilon_{s}^{1}-p_{s}^{1} \epsilon_{r}^{1}\right)\left(p_{s}^{4} \epsilon_{w}^{4}-p_{w}^{4} \epsilon_{s}^{4}\right)\left(p_{r}^{2} \epsilon_{u}^{2}-p_{u}^{2} \epsilon_{r}^{2}\right)\left(p_{q}^{3} \epsilon_{w}^{3}-p_{w}^{3} \epsilon_{q}^{3}\right)\right] \\
& \left.+\mathcal{F}^{G_{3,9}}(u, s)[2 \leftrightarrow 3]+\mathcal{F}^{G_{3,9}}(s, t)[2 \leftrightarrow 4]\right) .
\end{aligned}
$$

The most general descendant Lagrangian giving rise to (K.40) is

$$
\begin{aligned}
L^{G_{3}, 9}= & \sum_{m, n}\left(\mathcal{F}^{G_{3}, 9}\right)_{m, n} 2^{m+n} \\
& \times\left(\prod_{i=1}^{m} \prod_{j=1}^{n}\left(\partial_{\mu_{i}} \partial_{\nu_{j}} R_{p q r s}\right) R_{p t r u}\left(\partial^{\mu_{i}} R_{t v q w}\right)\left(\partial^{\nu_{j}} R_{u v s w}\right)\right)
\end{aligned}
$$


It is easy to see that the descendant Lagrangian (K.41) generates the S-matrix (K.40). Consider the Lagrangian

$$
R_{\text {pqrs }} R_{p t r u} R_{t v q w} R_{u v s w}
$$

which linearizes to give $F_{p q}^{1} F_{p t}^{2} F_{t v}^{3} F_{u v}^{4} F_{r u}^{2} F_{r s}^{1} F_{s w}^{4}$ plus permutations. The descendant Lagrangian (K.35) linearizes to give,

$$
\begin{aligned}
& \frac{1}{16} \sum_{m, n}\left(\mathcal{F}^{G_{3,9}}\right)_{m . n} 2^{m+n} \\
& \quad \times\left(\prod_{i=1}^{m} \prod_{j=1}^{n}\left(\partial_{\mu_{i}} \partial_{\nu_{j}} F_{p q}^{1} F_{r s}^{1}\right) F_{p t}^{2} F_{r u}^{2}\left(\partial^{\mu_{i}} F_{t v}^{3} F_{q w}^{3}\right)\left(\partial^{\nu_{j}} F_{u v}^{4} F_{s w}^{4}\right)\right)
\end{aligned}
$$

plus permutations.

With this new point of view, the most general gravitational S-matrix is now given by a sum of (K.22), (K.18), (K.10), (K.14), (K.1), (K.6), (K.30), (K.26), (K.34) and (K.40). However as we have explained in subsection 6.1.1, this way of listing the most general S-matrix - and most general Lagrangian - is 'over complete'. The fact that

$$
\begin{aligned}
r^{(i)} G_{\mathbf{S}, 1}=4 & \left(-G_{\mathbf{3}, 1}^{(i)}-2 G_{\mathbf{3}, 2}^{(i)}-16 G_{\mathbf{3}, 3}^{(i)}+16 G_{\mathbf{3}, 4}^{(i)}-2 G_{\mathbf{3}, 5}^{(i)}\right. \\
& \left.+10 G_{\mathbf{3}_{3,6}}^{(i)}+16 G_{\mathbf{3}_{3},}^{(i)}+\left(4 G_{\mathbf{3}_{\mathbf{A}}}^{(i+1)}-4 G_{\mathbf{3}_{\mathbf{A}}}^{(i+2)}\right)\right) .
\end{aligned}
$$

tells us (see (6.8)) that $\mathcal{F}$ 's obeys the following equivalence relation.

$$
\begin{aligned}
& \mathcal{F}^{G_{\mathbf{3}, 1}}(t, u) \sim \mathcal{F}^{G_{\mathbf{3}, 1}}(t, u)-(u-t) h(t, u), \\
& \mathcal{F}^{G_{\mathbf{3}, 2}}(t, u) \sim \mathcal{F}^{G_{\mathbf{3}, 2}}(t, u)-2(u-t) h(t, u), \\
& \mathcal{F}^{G_{\mathbf{3}, 3}}(t, u) \sim \mathcal{F}^{G_{\mathbf{3} 3}}(t, u)-16(u-t) h(t, u), \\
& \mathcal{F}^{G_{\mathbf{3}, 4}}(t, u) \sim \mathcal{F}^{G_{\mathbf{3}, 4}}(t, u)+16(u-t) h(t, u), \\
& \mathcal{F}^{G_{\mathbf{3}, 5}}(t, u) \sim \mathcal{F}^{G_{3,5}}(t, u)-2(u-t) h(t, u), \\
& \mathcal{F}^{G_{\mathbf{3}, 6}}(t, u) \sim \mathcal{F}^{G_{\mathbf{3}, 6}}(t, u)+10(u-t) h(t, u), \\
& \mathcal{F}^{G_{\mathbf{3}, 9}}(t, u) \sim \mathcal{F}^{G_{\mathbf{3}, 9}}(t, u)+16(u-t) h(t, u), \\
& \mathcal{F}^{G_{\mathbf{3}} \mathbf{A}}(t, u) \sim \mathcal{F}^{G_{\mathbf{3}_{\mathbf{A}}}(t, u)-4 s h(t, u) .}
\end{aligned}
$$

where $h(u, t)$ is a totally antisymmetric function under $S_{3}$. And also $\mathcal{F}^{G_{3,9}}(t, u)$ satisfies (K.39).

We can summarize the above discussion by stating that the general most general parity invariant s-matrix in $D \geq 7$ is given by

$$
S=\left(\sum_{i=1}^{8} S^{G_{3, i}}\right)+S^{G_{\mathbf{3}_{\mathbf{A}}}}+S^{G_{\mathbf{S}, 1}}+S^{G_{\mathbf{S}, 2}} .
$$

which is obtained from the general Lagrangian

$$
L=\left(\sum_{i=1}^{9} L^{G_{\mathbf{3}, i}}\right)+L^{G_{\mathbf{3}_{\mathbf{A}}}}+L^{G_{\mathbf{S}, 2}} .
$$




\section{K.2 $\quad D=7$}

Even. In $D=7$ the parity even S-matrices continue to be given by (K.46).

Odd. The most general S-matrix is specified by the momenta functions $\mathcal{F}^{H_{3,1}^{D}=7}(t, u)$ and $\mathcal{F}^{H_{\mathbf{3}, 2}^{D=7}}(t, u)$ that has $\mathbb{Z}_{2}$ symmetry under the two arguments and $\mathcal{F}^{H_{\mathbf{S}}^{D=7}}(t, u)$ that is completely symmetric under $S_{3}$.

$$
\begin{aligned}
S^{H_{3,1}^{D=7}}= & -4 i\left(\mathcal{F}^{H_{3,1}^{D=7}}(t, u)\left(p_{a}^{1} \epsilon_{b}^{1}-p_{b}^{1} \epsilon_{a}^{1}\right)\left(p_{b}^{2} \epsilon_{a}^{2}-p_{a}^{2} \epsilon_{b}^{2}\right)\left(p_{c}^{3} \epsilon_{d}^{3}-p_{d}^{3} \epsilon_{c}^{3}\right)\left(p_{d}^{4} \epsilon_{c}^{4}-p_{c}^{4} \epsilon_{d}^{4}\right)\right. \\
& +\mathcal{F}^{H_{3,1}^{D=7}}(s, u)\left(p_{a}^{1} \epsilon_{b}^{1}-p_{b}^{1} \epsilon_{a}^{1}\right)\left(p_{b}^{3} \epsilon_{a}^{3}-p_{a}^{3} \epsilon_{b}^{3}\right)\left(p_{c}^{2} \epsilon_{d}^{2}-p_{d}^{2} \epsilon_{c}^{2}\right)\left(p_{d}^{4} \epsilon_{c}^{4}-p_{c}^{4} \epsilon_{d}^{4}\right) \\
& \left.+\mathcal{F}^{H_{3,1}^{D=7}}(t, s)\left(p_{a}^{1} \epsilon_{b}^{1}-p_{b}^{1} \epsilon_{a}^{1}\right)\left(p_{b}^{4} \epsilon_{a}^{4}-p_{a}^{4} \epsilon_{b}^{4}\right)\left(p_{c}^{3} \epsilon_{d}^{3}-p_{d}^{3} \epsilon_{c}^{3}\right)\left(p_{d}^{2} \epsilon_{c}^{2}-p_{c}^{2} \epsilon_{d}^{2}\right)\right) \\
& \times\left(*\left(8 \epsilon^{1} \wedge p^{2} \wedge \epsilon^{2} \wedge p^{3} \wedge \epsilon^{3} \wedge p^{4} \wedge \epsilon^{4}\right)\right) \\
S^{H_{3,2}^{D=7}}= & -4 i\left(\mathcal{F}^{H_{3,2}^{D}=}(s, u)\left(p_{a}^{1} \epsilon_{b}^{1}-p_{b}^{1} \epsilon_{a}^{1}\right)\left(p_{b}^{2} \epsilon_{c}^{2}-p_{c}^{2} \epsilon_{b}^{2}\right)\left(p_{c}^{3} \epsilon_{d}^{3}-p_{d}^{3} \epsilon_{c}^{3}\right)\left(p_{d}^{4} \epsilon_{a}^{4}-p_{a}^{4} \epsilon_{d}^{4}\right)\right. \\
& +\mathcal{F}^{H_{3,2}^{D=7}}(t, s)\left(p_{a}^{1} \epsilon_{b}^{1}-p_{b}^{1} \epsilon_{a}^{1}\right)\left(p_{b}^{3} \epsilon_{c}^{3}-p_{c}^{3} \epsilon_{b}^{3}\right)\left(p_{c}^{4} \epsilon_{d}^{4}-p_{d}^{4} \epsilon_{c}^{4}\right)\left(p_{d}^{2} \epsilon_{a}^{2}-p_{a}^{2} \epsilon_{d}^{2}\right) \\
& \left.+\mathcal{F}^{H_{3,2}^{D=7}}(u, t)\left(p_{a}^{1} \epsilon_{b}^{1}-p_{b}^{1} \epsilon_{a}^{1}\right)\left(p_{b}^{4} \epsilon_{c}^{4}-p_{c}^{4} \epsilon_{b}^{4}\right)\left(p_{c}^{2} \epsilon_{d}^{2}-p_{d}^{2} \epsilon_{c}^{2}\right)\left(p_{d}^{3} \epsilon_{a}^{3}-p_{a}^{3} \epsilon_{d}^{3}\right)\right) \\
& \times\left(8 *\left(\epsilon^{1} \wedge p^{2} \wedge \epsilon^{2} \wedge p^{3} \wedge \epsilon^{3} \wedge p^{4} \wedge \epsilon^{4}\right)\right) \\
S^{H_{\mathbf{S}}^{D=7}}= & 4 i\left(\mathcal{F}^{H_{\mathbf{S}}^{D=7}}(t, u)\right) \\
& \times\left(\left(p_{\alpha}^{1} \epsilon_{\beta}^{1}-p_{\beta}^{1} \epsilon_{\alpha}^{1}\right) p_{\alpha}^{2}\left(p_{\gamma}^{2} \epsilon_{\delta}^{2}-p_{\delta}^{2} \epsilon_{\gamma}^{2}\right) p_{\beta}^{3}\left(p_{\delta}^{3} \epsilon_{\mu}^{3}-p_{\mu}^{3} \epsilon_{\delta}^{3}\right)\left(p_{\delta}^{4} \epsilon_{\gamma}^{4}-p_{\gamma}^{4} \epsilon_{\delta}^{4}\right)\right. \\
& +\left(p_{\alpha}^{2} \epsilon_{\beta}^{2}-p_{\beta}^{2} \epsilon_{\alpha}^{2}\right) p_{\alpha}^{1}\left(p_{\gamma}^{1} \epsilon_{\delta}^{1}-p_{\delta}^{1} \epsilon_{\gamma}^{1}\right) p_{\beta}^{3}\left(p_{\delta}^{3} \epsilon_{\mu}^{3}-p_{\mu}^{3} \epsilon_{\delta}^{3}\right)\left(p_{\delta}^{4} \epsilon_{\gamma}^{4}-p_{\gamma}^{4} \epsilon_{\delta}^{4}\right) \\
& +\left(p_{\alpha}^{3} \epsilon_{\beta}^{3}-p_{\beta}^{3} \epsilon_{\alpha}^{3}\right) p_{\alpha}^{2}\left(p_{\gamma}^{2} \epsilon_{\delta}^{2}-p_{\delta}^{2} \epsilon_{\gamma}^{2}\right) p_{\beta}^{1}\left(p_{\delta}^{1} \epsilon_{\mu}^{1}-p_{\mu}^{1} \epsilon_{\delta}^{1}\right)\left(p_{\delta}^{4} \epsilon_{\gamma}^{4}-p_{\gamma}^{4} \epsilon_{\delta}^{4}\right) \\
& \left.+\left(p_{\alpha}^{4} \epsilon_{\beta}^{4}-p_{\beta}^{4} \epsilon_{\alpha}^{4}\right) p_{\alpha}^{2}\left(p_{\gamma}^{2} \epsilon_{\delta}^{2}-p_{\delta}^{2} \epsilon_{\gamma}^{2}\right) p_{\beta}^{3}\left(p_{\delta}^{3} \epsilon_{\mu}^{3}-p_{\mu}^{3} \epsilon_{\delta}^{3}\right)\left(p_{\delta}^{1} \epsilon_{\gamma}^{1}-p_{\gamma}^{1} \epsilon_{\delta}^{1}\right)\right) \\
& \times\left(8 *\left(\epsilon^{1} \wedge p^{2} \wedge \epsilon^{2} \wedge p^{3} \wedge \epsilon^{3} \wedge p^{4} \wedge \epsilon^{4}\right)\right)
\end{aligned}
$$

The Lagrangians generating S-matrix (K.48) are listed in (6.2.1)

\section{K.3 $D=6$}

Even. As mentioned in the main text, the most general S-matrix and Lagrangian continue to be given by (K.46) and (K.47) but with the function $\mathcal{F}^{G_{\mathbf{S}, 1}}(t, u)$ set equal to zero.

Odd. The most general parity odd S-matrix in $D=6$ is specified by the momenta functions $\mathcal{F}^{H_{\mathbf{A}}^{D}, 1}(t, u), \mathcal{F}^{H_{\mathbf{3}_{\mathbf{A}}, 2}^{D=6}}(t, u)$ and $\mathcal{F}^{H_{\mathbf{3}_{\mathbf{A}}, 3}^{D=6}}(t, u)$ all of which are antisymmetric in first two arguments. All three structures transform in $\mathbf{3}_{\mathbf{A}}=\mathbf{2}_{\mathbf{M}} \oplus \mathbf{1}_{\mathbf{A}}$. 
The Explicit S-matrix is as follows, dropping the $D=6$ superscript.

$$
\begin{aligned}
& S^{H_{\mathbf{3}_{\mathbf{A}}, 1}}=\frac{1}{16}\left(\mathcal{F}^{H_{\mathbf{3}_{\mathbf{A}}, 1}}(t, u)\left(p_{a}^{1} \epsilon_{b}^{1}-p_{b}^{1} \epsilon_{a}^{1}\right)\left(p_{b}^{2} \epsilon_{a}^{2}-p_{a}^{2} \epsilon_{b}^{2}\right)\left(p_{c}^{3} \epsilon_{d}^{3}-p_{d}^{3} \epsilon_{c}^{3}\right)\left(p_{d}^{4} \epsilon_{c}^{4}-p_{c}^{4} \epsilon_{d}^{4}\right)\right. \\
& +\mathcal{F}^{H_{\mathbf{3}_{\mathbf{A}}, 1}}(s, u)\left(p_{a}^{1} \epsilon_{b}^{1}-p_{b}^{1} \epsilon_{a}^{1}\right)\left(p_{b}^{3} \epsilon_{a}^{3}-p_{a}^{3} \epsilon_{b}^{3}\right)\left(p_{c}^{2} \epsilon_{d}^{2}-p_{d}^{2} \epsilon_{c}^{2}\right)\left(p_{d}^{4} \epsilon_{c}^{4}-p_{c}^{4} \epsilon_{d}^{4}\right) \\
& \left.\times \mathcal{F}^{H_{\mathbf{3}_{\mathbf{A}}, 1}}(t, s)\left(p_{a}^{1} \epsilon_{b}^{1}-p_{b}^{1} \epsilon_{a}^{1}\right)\left(p_{b}^{4} \epsilon_{a}^{4}-p_{a}^{4} \epsilon_{b}^{4}\right)\left(p_{c}^{3} \epsilon_{d}^{3}-p_{d}^{3} \epsilon_{c}^{3}\right)\left(p_{d}^{2} \epsilon_{c}^{2}-p_{c}^{2} \epsilon_{d}^{2}\right)\right) \\
& \times\left(8\left(p_{\alpha}^{1} \epsilon_{\beta}^{1}-p_{\beta}^{1} \epsilon_{\alpha}^{1}\right) p_{\alpha}^{2} p_{\beta}^{3} *\left(\epsilon^{2} \wedge k^{2} \wedge \epsilon^{3} \wedge k^{3} \wedge \epsilon^{4} \wedge k^{4}\right)\right. \\
& +8\left(p_{\alpha}^{2} \epsilon_{\beta}^{2}-p_{\beta}^{2} \epsilon_{\alpha}^{2}\right) p_{\alpha}^{1} p_{\beta}^{3} *\left(\epsilon^{1} \wedge k^{1} \wedge \epsilon^{3} \wedge k^{3} \wedge \epsilon^{4} \wedge k^{4}\right) \\
& +8\left(p_{\alpha}^{3} \epsilon_{\beta}^{3}-p_{\beta}^{3} \epsilon_{\alpha}^{3}\right) p_{\alpha}^{2} p_{\beta}^{1} *\left(\epsilon^{2} \wedge k^{2} \wedge \epsilon^{1} \wedge k^{1} \wedge \epsilon^{4} \wedge k^{4}\right) \\
& \left.\times 8\left(p_{\alpha}^{4} \epsilon_{\beta}^{4}-p_{\beta}^{4} \epsilon_{\alpha}^{4}\right) p_{\alpha}^{2} p_{\beta}^{3} *\left(\epsilon^{2} \wedge k^{2} \wedge \epsilon^{3} \wedge k^{3} \wedge \epsilon^{1} \wedge k^{1}\right)\right) \\
& S^{H_{\mathbf{3}_{\mathbf{A}}, 2}}=\frac{1}{16}\left(\mathcal{F}^{H_{\mathbf{3}_{\mathbf{A}}, 2}}(s, u)\left(p_{a}^{1} \epsilon_{b}^{1}-p_{b}^{1} \epsilon_{a}^{1}\right)\left(p_{b}^{2} \epsilon_{c}^{2}-p_{c}^{2} \epsilon_{b}^{2}\right)\left(p_{c}^{3} \epsilon_{d}^{3}-p_{d}^{3} \epsilon_{c}^{3}\right)\left(p_{d}^{4} \epsilon_{a}^{4}-p_{a}^{4} \epsilon_{d}^{4}\right)\right. \\
& +\mathcal{F}^{H_{\mathbf{3}_{\mathbf{A}}, 2}}(t, s)\left(p_{a}^{1} \epsilon_{b}^{1}-p_{b}^{1} \epsilon_{a}^{1}\right)\left(p_{b}^{3} \epsilon_{c}^{3}-p_{c}^{3} \epsilon_{b}^{3}\right)\left(p_{c}^{4} \epsilon_{d}^{4}-p_{d}^{4} \epsilon_{c}^{4}\right)\left(p_{d}^{2} \epsilon_{a}^{2}-p_{a}^{2} \epsilon_{d}^{2}\right) \\
& \left.\times \mathcal{F}^{H_{3_{\mathbf{A}}, 2}}(u, t)\left(p_{a}^{1} \epsilon_{b}^{1}-p_{b}^{1} \epsilon_{a}^{1}\right)\left(p_{b}^{4} \epsilon_{c}^{4}-p_{c}^{4} \epsilon_{b}^{4}\right)\left(p_{c}^{2} \epsilon_{d}^{2}-p_{d}^{2} \epsilon_{c}^{2}\right)\left(p_{d}^{3} \epsilon_{a}^{3}-p_{a}^{3} \epsilon_{d}^{3}\right)\right) \\
& \times\left(8\left(p_{\alpha}^{1} \epsilon_{\beta}^{1}-p_{\beta}^{1} \epsilon_{\alpha}^{1}\right) p_{\alpha}^{2} p_{\beta}^{3} *\left(\epsilon^{2} \wedge k^{2} \wedge \epsilon^{3} \wedge k^{3} \wedge \epsilon^{4} \wedge k^{4}\right)\right. \\
& +8\left(p_{\alpha}^{2} \epsilon_{\beta}^{2}-p_{\beta}^{2} \epsilon_{\alpha}^{2}\right) p_{\alpha}^{1} p_{\beta}^{3} *\left(\epsilon^{1} \wedge k^{1} \wedge \epsilon^{3} \wedge k^{3} \wedge \epsilon^{4} \wedge k^{4}\right) \\
& +8\left(p_{\alpha}^{3} \epsilon_{\beta}^{3}-p_{\beta}^{3} \epsilon_{\alpha}^{3}\right) p_{\alpha}^{2} p_{\beta}^{1} *\left(\epsilon^{2} \wedge k^{2} \wedge \epsilon^{1} \wedge k^{1} \wedge \epsilon^{4} \wedge k^{4}\right) \\
& \left.\times 8\left(p_{\alpha}^{4} \epsilon_{\beta}^{4}-p_{\beta}^{4} \epsilon_{\alpha}^{4}\right) p_{\alpha}^{2} p_{\beta}^{3} *\left(\epsilon^{2} \wedge k^{2} \wedge \epsilon^{3} \wedge k^{3} \wedge \epsilon^{1} \wedge k^{1}\right)\right) \\
& S^{H_{\mathbf{B}_{\mathbf{A}}, 3}}=\frac{1}{16}\left(\mathcal { F } ^ { H _ { \mathbf { 3 } _ { \mathbf { A } } , 3 } } ( s , t ) \left(\epsilon^{a b c d e f}\left(p_{\gamma}^{1} \epsilon_{\delta}^{1}-p_{\delta}^{1} \epsilon_{\gamma}^{1}\right)\left(p_{e}^{2} \epsilon_{\rho}^{2}-p_{\rho}^{2} \epsilon_{e}^{2}\right)\left(p_{f}^{3} \epsilon_{\rho}^{3}-p_{\rho}^{3} \epsilon_{f}^{3}\right)\left(p_{\delta}^{4} \epsilon_{\gamma}^{4}-p_{\gamma}^{4} \epsilon_{\delta}^{4}\right)\right.\right. \\
& \times\left(\left(p_{a}^{1} \epsilon_{b}^{1}-p_{b}^{1} \epsilon_{a}^{1}\right) k_{c}^{2}\left(p_{\mu}^{2} \epsilon_{\nu}^{2}-p_{\nu}^{2} \epsilon_{\mu}^{2}\right) k_{d}^{3}\left(p_{\nu}^{3} \epsilon_{\alpha}^{3}-p_{\alpha}^{3} \epsilon_{\nu}^{3}\right)\left(p_{\alpha}^{4} \epsilon_{\mu}^{4}-p_{\mu}^{4} \epsilon_{\alpha}^{4}\right)-\left(p_{a}^{4} \epsilon_{b}^{4}-p_{b}^{4} \epsilon_{a}^{4}\right)\right. \\
& \left.\times k_{c}^{3}\left(p_{\mu}^{3} \epsilon_{\nu}^{3}-p_{\nu}^{3} \epsilon_{\mu}^{3}\right) k_{d}^{2}\left(p_{\nu}^{2} \epsilon_{\alpha}^{2}-p_{\alpha}^{2} \epsilon_{\nu}^{2}\right)\left(p_{\alpha}^{1} \epsilon_{\mu}^{1}-p_{\mu}^{1} \epsilon_{\alpha}^{1}\right)\right) \\
& +\left(p_{\gamma}^{2} \epsilon_{\delta}^{2}-p_{\delta}^{2} \epsilon_{\gamma}^{2}\right)\left(p_{e}^{1} \epsilon_{\rho}^{1}-p_{\rho}^{1} \epsilon_{e}^{1}\right)\left(p_{f}^{4} \epsilon_{\rho}^{4}-p_{\rho}^{4} \epsilon_{f}^{4}\right)\left(p_{\delta}^{3} \epsilon_{\gamma}^{3}-p_{\gamma}^{3} \epsilon_{\delta}^{3}\right) \\
& \times\left(\left(p_{a}^{2} \epsilon_{b}^{2}-p_{b}^{2} \epsilon_{a}^{2}\right) k_{c}^{1}\left(p_{\mu}^{1} \epsilon_{\nu}^{1}-p_{\nu}^{1} \epsilon_{\mu}^{1}\right) k_{d}^{4}\left(p_{\nu}^{4} \epsilon_{\alpha}^{4}-p_{\alpha}^{4} \epsilon_{\nu}^{4}\right)\left(p_{\alpha}^{3} \epsilon_{\mu}^{3}-p_{\mu}^{3} \epsilon_{\alpha}^{3}\right)-\left(p_{a}^{3} \epsilon_{b}^{3}-p_{b}^{3} \epsilon_{a}^{3}\right)\right. \\
& \left.\left.\times k_{c}^{4}\left(p_{\mu}^{4} \epsilon_{\nu}^{4}-p_{\nu}^{4} \epsilon_{\mu}^{4}\right) k_{d}^{1}\left(p_{\nu}^{1} \epsilon_{\alpha}^{1}-p_{\alpha}^{1} \epsilon_{\nu}^{1}\right)\left(p_{\alpha}^{2} \epsilon_{\mu}^{2}-p_{\mu}^{2} \epsilon_{\alpha}^{2}\right)\right)\right) \\
& \left.+\mathcal{F}^{H_{\mathbf{3}_{\mathbf{A}}, 3}}(u, t)(2 \leftrightarrow 4)+\mathcal{F}^{H_{\mathbf{3}_{\mathbf{A}}, 3}}(s, u)(3 \leftrightarrow 4)\right)
\end{aligned}
$$

The most general descendant Lagrangian which gives rise to (K.49) is given by

$$
\begin{aligned}
L^{H_{\mathbf{B}_{\mathbf{A}}, 1}}= & -\sum_{m, n}\left(\mathcal{F}^{H_{\mathbf{3}_{\mathbf{A}}, 1}}\right)_{m, n} 2^{m+n} \\
& \times\left(\prod_{i=1}^{m} \prod_{j=1}^{n} \epsilon^{a b c d e f}\left(\partial_{\mu_{i}} \partial_{\nu_{j}} R_{\mu \nu \alpha \beta}\right) \partial_{\alpha} R_{\nu \mu a b} \partial^{\mu_{i}} \partial_{\beta} R_{\gamma \delta c d} \partial^{\nu_{j}} R_{\delta \gamma e f}\right), \\
L^{H_{\mathbf{3}_{\mathbf{A}}, 2}}= & -\sum_{m, n}\left(\mathcal{F}^{H_{\mathbf{3}_{\mathbf{A}}, 2}}\right)_{m . n} 2^{m+n} \\
& \times\left(\prod_{i=1}^{m} \prod_{j=1}^{n} \epsilon^{a b c d e f}\left(\partial_{\mu_{i}} \partial_{\nu_{j}} R_{\mu \nu \alpha \beta}\right) \partial^{\mu_{i}} \partial_{\alpha} R_{\nu \gamma a b} \partial_{\beta} R_{\gamma \delta c d} \partial^{\nu_{j}} R_{\delta \mu e f}\right),
\end{aligned}
$$




$$
\begin{aligned}
L^{H_{\mathbf{3}_{\mathbf{A}}, 3}=} & -\sum_{m, n}\left(\mathcal{F}^{\left.H_{\mathbf{3}_{\mathbf{A}}, 3}\right)_{m . n}} 2^{m+n}\right. \\
& \times\left(\prod_{i=1}^{m} \prod_{j=1}^{n} \epsilon^{a b c d e f}\left(\partial_{\mu_{i}} \partial_{\nu_{j}} R_{a b \gamma \delta}\right)\left(\partial^{\mu_{i}} \partial_{c} R_{\mu \nu e \rho}\right)\left(\partial^{\nu_{j}} \partial_{d} R_{\nu \alpha f \rho}\right) R_{\alpha \mu \delta \gamma}\right),
\end{aligned}
$$

Consider the following Lagrangian structures,

$$
\begin{aligned}
& \epsilon^{a b c d e f} R_{\mu \nu \alpha \beta} \partial_{\alpha} R_{\nu \mu a b} \partial_{\beta} R_{\gamma \delta c d} R_{\delta \gamma e f}, \quad \epsilon^{a b c d e f} R_{\mu \nu \alpha \beta} \partial_{\alpha} R_{\nu \gamma a b} \partial_{\beta} R_{\gamma \delta c d} R_{\delta \mu e f} \\
& \epsilon^{a b c d e f} R_{a b \gamma \delta} \partial_{c} R_{\mu \nu e \rho} \partial_{d} R_{\nu \alpha f \rho} R_{\alpha \mu \delta \gamma} .
\end{aligned}
$$

The linearized structure in momentum space is proportional to

$$
\begin{aligned}
& \frac{1}{16}\left(\operatorname{Tr}\left(F^{1} F^{2}\right) \operatorname{Tr}\left(F^{3} F^{4}\right)\right)\left(\epsilon^{a b c d e f} F_{\alpha \beta}^{1} \partial^{\alpha} F_{a b}^{2} \partial^{\beta} F_{c d}^{3} F_{e f}^{4}\right), \\
& \frac{1}{16}\left(\operatorname{Tr}\left(F^{1} F^{2} F^{3} F^{4}\right)\right)\left(\epsilon^{a b c d e f} F_{\alpha \beta}^{1} \partial^{\alpha} F_{a b}^{2} \partial^{\beta} F_{c d}^{3} F_{e f}^{4}\right) \\
& \frac{1}{16} \epsilon^{a b c d e f} F_{a b}^{1}\left(\partial_{c} F_{\mu \nu}^{2} \partial_{d} F_{\nu \alpha}^{3} F_{\alpha \mu}^{4}\right)\left(F_{\gamma \delta}^{1} F_{e \rho}^{2} F_{f \rho}^{3} F_{\delta \gamma}^{4}\right) .
\end{aligned}
$$

plus permutations. It is therefore easy to see that the descendant Lagrangian (K.50) gives rise to S-matrix (K.49).

\section{K.4 $D=5$}

Even. There is further reduction of $2 \mathbb{Z}_{2}$ symmetric structures in this dimension. To be precise there all the Lagrangians giving rise to the labelled Lagrangians $G_{\mathbf{3}, 1}-G_{\mathbf{3}, 6}$ are not linearly independent. We find the following relation between the s-matrices.

$$
\sum_{i=2}^{6} S^{G_{3, i}}=0
$$

with

$$
\begin{aligned}
& \mathcal{F}^{G_{3,6}}(t, u)=\frac{5 \alpha}{2}-2 \beta, \quad \mathcal{F}^{G_{3,5}}(t, u)=-\frac{\alpha}{2}, \quad \mathcal{F}^{G_{\mathbf{3}, 3}}(t, u)=2 \beta \\
& \mathcal{F}^{G_{3,4}}(t, u)=2 \alpha, \quad \mathcal{F}^{G_{3,1}}(t, u)=\frac{-6 \alpha+5 \beta}{8} .
\end{aligned}
$$

The structures we choose to reduce: $L^{G_{3,3}}$ and $L^{G_{3,4}}$.

Odd. The most general parity odd S-matrix in $D=5$ is specified by the momentum function $\mathcal{F}^{H_{\mathbf{A}}^{D=5}}(t, u)$ which transforms in $\mathbf{2}_{\mathbf{M}}+\mathbf{1}_{\mathbf{A}}=\mathbf{3}_{\mathbf{A}}$ of $S_{3}$. As a result this function is antisymmetric in the two arguments. 
The explicit S-matrix is as follows,

$$
\begin{aligned}
S^{H_{\mathbf{3}}^{D=5}}= & -i\left(\mathcal { F } ^ { H _ { \mathbf { 3 } _ { \mathbf { A } } = 5 } ^ { D } } ( s , t ) \left(4 *\left(p^{4} \wedge \epsilon^{4} \wedge p^{1} \wedge \epsilon^{1} \wedge p^{2}\right)\left(p_{f}^{2} \epsilon_{g}^{2}-p_{g}^{2} \epsilon_{f}^{2}\right)\left(p_{g}^{3} \epsilon_{h}^{3}-p_{h}^{3} \epsilon_{g}^{3}\right)\left(p_{h}^{4} \epsilon_{f}^{4}-p_{f}^{4} \epsilon_{h}^{4}\right)\right.\right. \\
& \times\left(p_{\alpha}^{1} \epsilon_{\beta}^{1}-p_{\beta}^{1} \epsilon_{\alpha}^{1}\right) p_{\alpha}^{2} p_{\beta}^{3}\left(p_{\mu}^{2} \epsilon_{\nu}^{2}-p_{\nu}^{2} \epsilon_{\mu}^{2}\right)\left(p_{\nu}^{3} \epsilon_{\mu}^{3}-p_{\mu}^{3} \epsilon_{\nu}^{3}\right)+(1 \leftrightarrow 2,3 \leftrightarrow 4)+(1 \leftrightarrow 3,3 \leftrightarrow 4) \\
& +(1 \leftrightarrow 4,2 \leftrightarrow 3)) \\
& +\mathcal{F}^{H_{\mathbf{3}_{\mathbf{A}}^{D}=5}^{D}}(t, u)\left(4 *\left(p^{2} \wedge \epsilon^{2} \wedge p^{1} \wedge \epsilon^{1} \wedge p^{3}\right)\left(p_{f}^{3} \epsilon_{g}^{3}-p_{g}^{3} \epsilon_{f}^{3}\right)\left(p_{g}^{4} \epsilon_{h}^{4}-p_{h}^{4} \epsilon_{g}^{4}\right)\left(p_{h}^{2} \epsilon_{f}^{2}-p_{f}^{2} \epsilon_{h}^{2}\right)\right. \\
& \times\left(p_{\alpha}^{1} \epsilon_{\beta}^{1}-p_{\beta}^{1} \epsilon_{\alpha}^{1}\right) p_{\alpha}^{3} p_{\beta}^{4}\left(p_{\mu}^{3} \epsilon_{\nu}^{3}-p_{\nu}^{3} \epsilon_{\mu}^{3}\right)\left(p_{\nu}^{4} \epsilon_{\mu}^{4}-p_{\mu}^{4} \epsilon_{\nu}^{4}\right)+(1 \leftrightarrow 2,3 \leftrightarrow 4)+(1 \leftrightarrow 3,3 \leftrightarrow 4) \\
& +(1 \leftrightarrow 4,2 \leftrightarrow 3)) \\
& +\mathcal{F}^{H_{\mathbf{3}_{\mathbf{A}}=5}}(u, s)\left(4 *\left(p^{3} \wedge \epsilon^{3} \wedge p^{1} \wedge \epsilon^{1} \wedge p^{4}\right)\left(p_{f}^{4} \epsilon_{g}^{4}-p_{g}^{4} \epsilon_{f}^{4}\right)\left(p_{g}^{2} \epsilon_{h}^{2}-p_{h}^{2} \epsilon_{g}^{2}\right)\left(p_{h}^{3} \epsilon_{f}^{3}-p_{f}^{3} \epsilon_{h}^{3}\right)\right. \\
& \times\left(p_{\alpha}^{1} \epsilon_{\beta}^{1}-p_{\beta}^{1} \epsilon_{\alpha}^{1}\right) p_{\alpha}^{4} p_{\beta}^{2}\left(p_{\mu}^{4} \epsilon_{\nu}^{4}-p_{\nu}^{4} \epsilon_{\mu}^{4}\right)\left(p_{\nu}^{2} \epsilon_{\mu}^{2}-p_{\mu}^{2} \epsilon_{\nu}^{2}\right)+(1 \leftrightarrow 2,3 \leftrightarrow 4)+(1 \leftrightarrow 3,3 \leftrightarrow 4) \\
& +(1 \leftrightarrow 4,2 \leftrightarrow 3)))
\end{aligned}
$$

The S-matrix (K.55) is obtained from the following descendant Lagrangian The most general descendant is given by

$$
\begin{aligned}
L^{H_{\mathbf{3}}^{D=5}}= & \sum_{m, n}\left(\mathcal{F}^{H_{\mathbf{3}_{\mathbf{A}}=5}^{D=5}}\right)_{m, n} 2^{m+n} \\
& \times\left(\prod_{i=1}^{m} \prod_{j=1}^{n} \epsilon^{a b c d e}\left(\left(\partial_{\mu_{i}} \partial_{\nu_{j}} R_{\alpha \beta c d}\right) \partial^{\mu_{i}} \partial_{\alpha} \partial_{e} R_{\mu \nu f g} \partial^{\nu_{j}} \partial_{\beta} R_{\nu \mu g h} R_{a b h f}\right)\right.
\end{aligned}
$$

The $\mathbb{Z}_{2}$ antisymmetry of the momentum function $\mathcal{F}^{H_{\mathbf{B}_{\mathrm{A}}}^{D=5}}(t, u)$ is explicit in momentum space and can be seen by re-labelling the second and the third Riemann tensor in (K.56) and using conservation equation.

That the S-matrix (K.55) is obtained from the Lagrangian (K.56) is evident when one considers the Lagrangian

$$
\epsilon^{a b c d e} R_{\alpha \beta c d} \partial_{\alpha} \partial_{e} R_{\mu \nu f g} \partial_{\beta} R_{\nu \mu g h} R_{a b h f}
$$

The linearized structure in momentum space is given by,

$$
\epsilon^{a b c d e}\left(F_{\alpha \beta}^{1} \partial_{\alpha} F_{\mu \nu}^{2} \partial_{\beta} F_{\nu \mu}^{3} F_{a b}^{4}\right)\left(F_{c d}^{1} \partial_{e} F_{f g}^{2} F_{g h}^{3} F_{h f}^{4}\right)
$$

plus permutations.

\section{K.5 $D=4$}

Even. The independent parity even structures are just three.

$$
\begin{aligned}
& \left(R_{a b c d} R_{a b c d}\right)^{2}, \quad R_{\text {pqrs }} R_{\text {pqtu }} R_{\text {rtvw }} R_{\text {suvw }} \\
& R_{a b c d} \nabla^{a} \nabla^{c} R_{e f e^{\prime} f^{\prime}} \nabla^{b} \nabla^{d} R_{f g f^{\prime} g^{\prime}} R_{\text {geg }} e^{\prime}
\end{aligned}
$$

We show the reduction of the local module as follows. From [40], we know that the Smatrix corresponding to $G_{\mathbf{6}}=G_{\mathbf{3}, 6} \oplus G_{\mathbf{3}_{\mathrm{A}}}$ vanishes. The relations that among the rest 8 
derivative Lagrangians are as follows.

$$
\begin{aligned}
S^{G_{\mathbf{3}, 5}}+S^{G_{\mathbf{3}, 1}}+S^{G_{\mathbf{3}, 2}} & =0 \\
\text { with, } \quad \mathcal{F}^{G_{\mathbf{3}, 5}}=1, \quad \mathcal{F}^{G_{\mathbf{3}, 1}} & =-\frac{1}{4}, \quad \mathcal{F}^{G_{\mathbf{3}, 2}}=\frac{1}{2} .
\end{aligned}
$$

We use this to eliminate $G_{\mathbf{3}, 2}$ from our basis. We also find linear relations between the s-matrix at 10 derivative level,

$$
\begin{aligned}
S^{G_{3,8}}+S^{G_{3,7}} & =0 \\
\text { with, } \quad \mathcal{F}^{G_{3,8}} & =-4, \quad \mathcal{F}^{G_{3,7}}=1 .
\end{aligned}
$$

Thus at 10 derivative level we only have $G_{\mathbf{3}, 7}$ as the basis structure. Finally we find the following relation between the descendants of $G_{\mathbf{3}, 1}, G_{\mathbf{3}, 5}$ and $G_{\mathbf{3}, 7}$.

$$
\begin{aligned}
S^{G_{3,7}}+S^{G_{3,1}}+S^{G_{3,5}} & =0 \\
\text { with } \quad \mathcal{F}^{G_{3,7}}=18, \quad \mathcal{F}^{G_{3,5}}(t, u) & =-4(t+u), \quad \mathcal{F}^{G_{3,1}}(t, u)=(t+u) .
\end{aligned}
$$

This can be used to eliminate $G_{\mathbf{3}, 7}$. Finally we are left with $G_{\mathbf{3}, 1}, G_{\mathbf{3}, 5}$ and $G_{\mathbf{3}, 9}$ as our local module generator in $D=4$. The most general parity even s-matrix is given by,

$$
S_{\text {even }}^{D=4}=S^{G_{3,1}}+S^{G_{3,5}}+S^{G_{3,9}} .
$$

Odd. The most general parity odd S-matrix in $D=4$ is parameterized by the momenta functions $\mathcal{F}_{3}^{O_{3}^{D=4}}(t, u)$ and $\mathcal{F}^{O O_{\mathrm{S}}^{D=4}}(t, u)$, where the first function is symmetric in the two arguments while the second one is completely symmetric under $S_{3}$. The explicit S-matrix is as follows,

$$
\begin{aligned}
& S^{O_{3}^{D=4}}=\frac{1}{8} \mathcal{F}^{O{ }_{3}^{D=4}}(t, u)\left(4 *\left(p_{1} \wedge \epsilon_{1} \wedge p_{2} \wedge \epsilon_{2}\right)\left(p_{\mu}^{3} \epsilon_{\nu}^{3}-p_{\nu}^{3} \epsilon_{\mu}^{3}\right)\left(p_{\mu}^{4} \epsilon_{\nu}^{4}-p_{\nu}^{4} \epsilon_{\mu}^{4}\right)\left(p_{a}^{1} \epsilon_{b}^{1}-p_{b}^{1} \epsilon_{a}^{1}\right)\right. \\
& \left.\times\left(p_{b}^{2} \epsilon_{a}^{2}-p_{a}^{2} \epsilon_{b}^{2}\right)\left(p_{c}^{3} \epsilon_{d}^{3}-p_{d}^{3} \epsilon_{c}^{3}\right)\left(p_{d}^{4} \epsilon_{c}^{4}-p_{c}^{4} \epsilon_{d}^{4}\right)+(1 \rightarrow 3,2 \rightarrow 4)\right) \\
& +\frac{1}{8} \mathcal{F}^{O O^{D=4}}(u, s)\left(4 *\left(p_{1} \wedge \epsilon_{1} \wedge p_{3} \wedge \epsilon_{3}\right)\left(p_{\mu}^{2} \epsilon_{\nu}^{2}-p_{\nu}^{2} \epsilon_{\mu}^{2}\right)\left(p_{\mu}^{4} \epsilon_{\nu}^{4}-p_{\nu}^{4} \epsilon_{\mu}^{4}\right)\left(p_{a}^{1} \epsilon_{b}^{1}-p_{b}^{1} \epsilon_{a}^{1}\right)\right. \\
& \left.\times\left(p_{b}^{3} \epsilon_{a}^{3}-p_{a}^{3} \epsilon_{b}^{2}\right)\left(p_{c}^{2} \epsilon_{d}^{2}-p_{d}^{2} \epsilon_{c}^{2}\right)\left(p_{d}^{4} \epsilon_{c}^{4}-p_{c}^{4} \epsilon_{d}^{4}\right)+(1 \rightarrow 2,3 \rightarrow 4)\right) \\
& +\frac{1}{8} \mathcal{F}^{O_{3}^{D=4}}(s, t)\left(4 *\left(p_{1} \wedge \epsilon_{1} \wedge p_{4} \wedge \epsilon_{4}\right)\left(p_{\mu}^{3} \epsilon_{\nu}^{3}-p_{\nu}^{3} \epsilon_{\mu}^{3}\right)\left(p_{\mu}^{2} \epsilon_{\nu}^{2}-p_{\nu}^{2} \epsilon_{\mu}^{2}\right)\left(p_{a}^{1} \epsilon_{b}^{1}-p_{b}^{1} \epsilon_{a}^{1}\right)\right. \\
& \left.\times\left(p_{b}^{4} \epsilon_{a}^{4}-p_{a}^{4} \epsilon_{b}^{4}\right)\left(p_{c}^{3} \epsilon_{d}^{3}-p_{d}^{3} \epsilon_{c}^{3}\right)\left(p_{d}^{2} \epsilon_{c}^{2}-p_{c}^{2} \epsilon_{d}^{2}\right)+(1 \rightarrow 2,3 \rightarrow 4)\right) \\
& S^{O O_{\mathrm{S}}^{D=4}}=\frac{1}{16}\left(\mathcal{F}^{O \mathrm{~S}=4}(t, u)\right) \\
& \times\left(2 *\left(p^{2} \wedge p^{3} \wedge p^{1} \wedge \epsilon^{1}\right)\left(p_{c}^{2} \epsilon_{d}^{2}-p_{d}^{2} \epsilon_{c}^{2}\right)\left(p_{d}^{3} \epsilon_{e}^{3}-p_{e}^{3} \epsilon_{d}^{3}\right)\left(p_{e}^{4} \epsilon_{c}^{4}-p_{c}^{4} \epsilon_{e}^{4}\right)\right. \\
& \times\left(p_{\alpha}^{1} \epsilon_{\beta}^{1}-p_{\beta}^{1} \epsilon_{\alpha}^{1}\right) p_{\alpha}^{2}\left(p_{\gamma}^{2} \epsilon_{\delta}^{2}-p_{\delta}^{2} \epsilon_{\gamma}^{2}\right) p_{\beta}^{3}\left(p_{\delta}^{3} \epsilon_{\mu}^{3}-p_{\mu}^{3} \epsilon_{\delta}^{3}\right)\left(p_{\delta}^{4} \epsilon_{\gamma}^{4}-p_{\gamma}^{4} \epsilon_{\delta}^{4}\right) \\
& +2 *\left(p^{1} \wedge p^{3} \wedge p^{2} \wedge \epsilon^{2}\right)\left(p_{c}^{1} \epsilon_{d}^{1}-p_{d}^{1} \epsilon_{c}^{1}\right)\left(p_{d}^{3} \epsilon_{e}^{3}-p_{e}^{3} \epsilon_{d}^{3}\right)\left(p_{e}^{4} \epsilon_{c}^{4}-p_{c}^{4} \epsilon_{e}^{4}\right) \\
& \times\left(p_{\alpha}^{2} \epsilon_{\beta}^{2}-p_{\beta}^{2} \epsilon_{\alpha}^{2}\right) p_{\alpha}^{1}\left(p_{\gamma}^{1} \epsilon_{\delta}^{1}-p_{\delta}^{1} \epsilon_{\gamma}^{1}\right) p_{\beta}^{3}\left(p_{\delta}^{3} \epsilon_{\mu}^{3}-p_{\mu}^{3} \epsilon_{\delta}^{3}\right)\left(p_{\delta}^{4} \epsilon_{\gamma}^{4}-p_{\gamma}^{4} \epsilon_{\delta}^{4}\right) \\
& +2 *\left(p^{2} \wedge p^{1} \wedge p^{3} \wedge \epsilon^{3}\right)\left(p_{c}^{2} \epsilon_{d}^{2}-p_{d}^{2} \epsilon_{c}^{2}\right)\left(p_{d}^{1} \epsilon_{e}^{1}-p_{e}^{1} \epsilon_{d}^{1}\right)\left(p_{e}^{4} \epsilon_{c}^{4}-p_{c}^{4} \epsilon_{e}^{4}\right) \\
& \times\left(p_{\alpha}^{3} \epsilon_{\beta}^{3}-p_{\beta}^{3} \epsilon_{\alpha}^{3}\right) p_{\alpha}^{2}\left(p_{\gamma}^{2} \epsilon_{\delta}^{2}-p_{\delta}^{2} \epsilon_{\gamma}^{2}\right) p_{\beta}^{1}\left(p_{\delta}^{1} \epsilon_{\mu}^{1}-p_{\mu}^{1} \epsilon_{\delta}^{1}\right)\left(p_{\delta}^{4} \epsilon_{\gamma}^{4}-p_{\gamma}^{4} \epsilon_{\delta}^{4}\right) \\
& +2 *\left(p^{2} \wedge p^{3} \wedge p^{4} \wedge \epsilon^{4}\right)\left(p_{c}^{2} \epsilon_{d}^{2}-p_{d}^{2} \epsilon_{c}^{2}\right)\left(p_{d}^{3} \epsilon_{e}^{3}-p_{e}^{3} \epsilon_{d}^{3}\right)\left(p_{e}^{1} \epsilon_{c}^{1}-p_{c}^{1} \epsilon_{e}^{1}\right) \\
& \left.\times\left(p_{\alpha}^{4} \epsilon_{\beta}^{4}-p_{\beta}^{4} \epsilon_{\alpha}^{4}\right) p_{\alpha}^{2}\left(p_{\gamma}^{2} \epsilon_{\delta}^{2}-p_{\delta}^{2} \epsilon_{\gamma}^{2}\right) p_{\beta}^{3}\left(p_{\delta}^{3} \epsilon_{\mu}^{3}-p_{\mu}^{3} \epsilon_{\delta}^{3}\right)\left(p_{\delta}^{1} \epsilon_{\gamma}^{1}-p_{\gamma}^{1} \epsilon_{\delta}^{1}\right)\right)
\end{aligned}
$$


The most general descendant corresponding to the S-matrix (K.64) is given by

$$
\begin{aligned}
L^{O_{3}^{D=4}}= & \sum_{m, n}\left(\mathcal{F}^{O_{3}^{D=4}}\right)_{m . n} 2^{m+n}\left(\prod_{i=1}^{m} \prod_{j=1}^{n} \epsilon^{a b i j}\left(\partial_{\mu_{i}} \partial_{\nu_{j}} R_{a b e f}\right) R_{i j f e} \partial^{\mu_{i}} R_{c d g h} \partial^{\nu_{j}} R_{d c h g}\right) \\
L^{O O_{\mathbf{S}}^{D=4}}= & \sum_{m, n}\left(\mathcal{F}^{O_{\mathbf{S}}^{D=4}}\right)_{m . n} 2^{m+n} \\
& \times\left(\prod_{i=1}^{m} \prod_{j=1}^{n} \epsilon^{a b m n}\left(\partial_{\mu_{i}} \partial_{\nu_{j}} R_{m n g h}\right) \partial^{\mu_{i}} \partial_{a} \partial_{g} R_{c d i j} \partial^{\nu_{j}} \partial_{b} \partial_{h} R_{d e j k} R_{e c k i}\right)
\end{aligned}
$$

It can be easily seen that the descendant Lagrangian (K.63) generates the Smatrix (K.64). Consider the following parity odd Lagrangians

$$
\epsilon^{a b i j} R_{a b e f} R_{i j f e} R_{c d g h} R_{d c h g}, \quad \epsilon^{a b m n} R_{m n g h} \partial^{a} \partial^{g} R_{c d i j} \partial^{b} \partial^{h} R_{d e j k} R_{e c k i} .
$$

Roughly these structures, when linearized, can be represented as the tensor product of the electromagnetism structures. The symmetries also become manifest.

$$
\begin{aligned}
& \operatorname{Tr}\left(F^{1} \wedge F^{2}\right) \operatorname{Tr}\left(F^{3} F^{4}\right)\left(\operatorname{Tr}\left(F^{1} F^{2}\right) \operatorname{Tr}\left(F^{3} F^{4}\right)\right), \\
& \left.\left(* F_{a b}^{1}\right) \operatorname{Tr}\left(\partial_{a} F^{2} \partial_{a} F^{3} F^{4}\right)\right)\left(\left(F_{a b}^{1}\right) \operatorname{Tr}\left(\partial_{a} F^{2} \partial_{a} F^{3} F^{4}\right)\right) .
\end{aligned}
$$

plus permutations. Therefore (K.63) generates the S-matrix (K.64).

\section{Sample exchange contributions to four point scattering}

\section{L.1 Most general exchange contribution to four scalar scattering}

Consider a massive particle in a representation $\mathcal{P}$ of its Lorentz little group $\mathrm{SO}(D-1)$. Such a particle can have a nonzero three point functions with either two scalars, two photons or two gravitons, only if $\mathcal{P}$ is vectorial rather than spinorial in nature. It follows that we can have exchange contributions to four scalar, four photon or four graviton scattering only when the exchanged particle transforms in a representation $\mathcal{P}$ that can be built by symmetrizing and anti-symmetrizing vectors (and then removing traces from the symmetric parts). Any such representation is labelled by a Young Tableaux.

Now consider a particle with momentum $p_{3}$ in such a representation of the little group. On-shell states of this particle are labelled by a polarization tensor, whose indices are all orthogonal to $p_{3}$ and are otherwise appropriately symmetrized/ anti-symmetrized and trace removed. Now consider the on-shell 3 point function between such a particle and two massless scalars with momentum $p_{1}$ and $p_{2}$. As the amplitude is Lorentz invariant, all indices of the polarization tensor associated with the particle $\mathcal{P}$ have to be contracted with $p_{1}-p_{2}$, the only vector available (recall that the polarization tensor is orthogonal to $p_{1}+p_{2}=-p_{3}$ ). As all indices of the polarization tensor are contracted with the same vector, it follows that the three point function vanishes unless $\mathcal{P}$ is labelled by a completely symmetric Young Tableaux, i.e. a Tableaux with a single row, i.e. is a traceless symmetric 
tensor, $e_{\mu_{1} \ldots \mu_{l}}$. In this case the three point function of two massless scalars with this representation is necessarily proportional to

$$
\left(p_{1}-p_{2}\right)_{1}^{\mu}\left(p_{1}-p_{2}\right)_{2}^{\mu} \ldots\left(p_{1}-p_{2}\right)_{l}^{\mu} e_{\mu_{1} \ldots \mu_{l}} .
$$

This three point function has the necessary Bose symmetry under interchange of 1 and 2 (and so is nonzero) only if $l$ is even.

The propagator for a massive spin $l$ particle is given by

$$
\frac{\mathcal{P}_{\alpha_{1} \alpha_{2} \ldots \alpha_{l}}^{\beta_{1} \beta_{2} \ldots \beta_{l}}}{p^{2}+m^{2}}
$$

where $\mathcal{P}$ is the projection operator that first projects all vector indices orthogonal to the momentum $p$ that runs through the propagator, and then projects the resulting $(D-1)^{l}$ dimensional Hilbert space (generated by the direct product of the $l$ vectors orthogonal to $p$ ) onto the subspace of spin $l$ traceless symmetric tensors. It follows that the scalar four point function that results from the exchange of a spin $l$ particle is proportional to

$$
\frac{\left(p_{1}-p_{2}\right)_{\alpha_{1}} \ldots\left(p_{1}-p_{2}\right)_{\alpha_{l}} \mathcal{P}_{\alpha_{1} \alpha_{2} \ldots \alpha_{l}}^{\beta_{1} \beta_{2} \ldots \beta_{l}}\left(p_{3}-p_{4}\right)_{\beta_{1}} \ldots\left(p_{3}-p_{4}\right)_{\beta_{l}}}{\left(p_{1}+p_{2}\right)^{2}+m^{2}}
$$

The explicit formula $[46,47]$ for the propagator $\mathcal{P}_{\alpha_{1} \alpha_{2} \ldots \alpha_{l}}^{\beta_{1} \beta_{2} \ldots \beta_{l}}$ is complicated by the requirement of tracelessness. This requirement determines the projector to be ${ }^{133}$

$$
\begin{aligned}
\mathcal{P}_{\mu_{1} \ldots \mu_{l}, \nu_{1} \ldots \nu_{l}}^{(s)}=\{ & \left\{\sum_{p=0}^{[l / 2]} \frac{(-1)^{p} l !(2 l+D-2 p-5) ! !}{2^{p} p !(l-2 p) !(2 l+D-5) ! !} \Theta_{\mu_{1} \mu_{2}} \Theta_{\nu_{1} \nu_{2}} \ldots \Theta_{\mu_{2 p-1} \mu_{2 p}} \Theta_{\nu_{2 p-1} \nu_{2 p}}\right. \\
& \left.\times \Theta_{\mu_{2 p+1} \nu_{2 p+1}} \ldots \Theta_{\mu_{s}, \nu_{s}}\right\}_{\operatorname{sym}(\mu), \operatorname{sym}(\nu)}
\end{aligned}
$$

where

$$
\Theta_{\mu \nu}=\eta_{\mu \nu}-p_{\mu} p_{\nu} / p^{2}
$$

is the projector onto the spatial slice orthogonal to $p_{\mu}$ and $[l / 2]$ is largest integer smaller than or equal to $l / 2$ and $\operatorname{sym}(\mu)$ denotes the operation of symmetrizing over the $l \mu$ indices, i.e the operation $\frac{1}{l !} \sum_{P}$ where the sum is over all $l$ ! permutations $P$ of the indices $\mu_{i}$ and $\operatorname{sym}(\nu)$ means something similar. Let us define

$$
\begin{aligned}
& a=\left(p_{1}-p_{2}\right)^{\mu} \Theta_{\mu \nu}\left(p_{1}-p_{2}\right)^{\nu} \\
& b=\left(p_{3}-p_{4}\right)^{\mu} \Theta_{\mu \nu}\left(p_{3}-p_{4}\right)^{\nu} \\
& c=\left(p_{1}-p_{2}\right)^{\mu} \Theta_{\mu \nu}\left(p_{3}-p_{4}\right)^{\nu}
\end{aligned}
$$

It follows from (L.1) and (L.2) that the spin $l$ exchange is given, up to a proportionality constant, by [46, 47] A simple computation yields

$$
\begin{aligned}
a & =s, \quad b=s, \quad c=u-t, \\
S & =\frac{(a b)^{\frac{l}{2}}}{\left(p_{1}+p_{2}\right)^{2}+m^{2}} A_{l}^{D}\left(\frac{c}{\sqrt{a b}}\right)+\text { crossing }
\end{aligned}
$$

\footnotetext{
${ }^{133}$ S.M. would like to thank P. Nayak, R. Sinha R. Soni and R. Poojari for detailed discussions - and initial collaboration on a related project [48] — on contributions to the $\mathrm{S}$ matrix of tree level exchanges to higher spin exchanges, that proved useful while writing this section.
} 
where we have defined the polynomial

$$
A_{l}^{D}(x) \equiv \sum_{p=0}^{[l / 2]} \frac{(-1)^{p} l !(2 l+D-2 p-5) ! !}{2^{p} p !(l-2 p) !(2 l+D-5) ! !}(x)^{l-2 p}=\frac{1}{2^{l}\left(\frac{D-3}{2}\right)_{l}} C_{l}^{\frac{D-3}{2}}(x), \quad(a)_{b} \equiv \frac{\Gamma(a+b)}{\Gamma(a)} .
$$

which is proportional to the Gegenbauer polynomial $C_{l}^{\frac{D-3}{2}}(x)$. Substituting (L.5) into (L.6) we obtain

$$
S \propto \frac{s^{l}}{m^{2}-s} A_{l}^{D}\left(\frac{u-t}{s}\right)+\frac{t^{l}}{m^{2}-t} A_{l}^{D}\left(\frac{s-u}{t}\right)+\frac{u^{l}}{m^{2}-u} A_{l}^{D}\left(\frac{t-s}{u}\right) .
$$

Regge growth. In the Regge limit the first and third lines in (L.8) - that correspond to $s$ channel and $u$ channel exchange - are qualitatively different from the second line $(t$ channel exchange).

The leading behavior of the $t$ channel graph is obtained from the term $p=0$ in the second line of (L.8). and is given by $\left(s^{l}\right) /\left(m^{2}-t\right)$. Note that this expression is not a polynomial in $t$. As 'impact parameter space' is roughly Fourier conjugate to $t$ space, it follows that $t$ channel exchange leads to non-trivial scattering at finite impact parameter in the Regge limit. Note the contrast with contact interactions (studied in detail earlier in this paper). As the S-matrices that follow from contact interactions are polynomial in $s$ as well as in $t$, such interactions contribute to scattering in the Regge limit only at zero impact parameter.

Note that, on the other hand, that at leading order in the Regge limit $s$ and $u$ channel scattering amplitudes are proportional to $s^{l-1}$ and $u^{l-1}$. Recall that since $l$ is even, the leading terms from the $\mathrm{s}$ and $\mathrm{u}$ channel cancel with each other. However the first subleading terms yield an S-matrix proportional to

$$
2 m^{2} s^{l-2}+3(l-2 p) s^{l-2} t
$$

This behavior is analytic in $t$ and so contributes only to scattering at zero impact parameter. The leading large $s$ behavior is of the sort that can be reproduced (and so also cancelled) by a local 'counter-term' whenever $l \geq 2$.

At any rate the second term of (L.8) gives the dominant growth of the S-matrix in the Regge limit, and this growth is proportional to $s^{l}$. Note in particular that the exchange of a massive spin zero scalar leads to scattering with Regge growth $s^{0}$, while the exchange of a massive spin 2 particle leads to an amplitude that grows in the Regge limit like $s^{2}$. All higher spin exchanges yield scattering amplitudes that grow faster than $s^{2}$ in the Regge limit. As the corresponding term is non analytic in $t$ it cannot be mimicked or cancelled by a local counter-term.

It is often asserted that scattering due to exchange of a spin $l$ particle leads to a scattering amplitude that grows like $s^{l}$ in the Regge limit. As we have already seen in the simple example of scalar scattering, this claim is really correct only in the $t$ channel - the channel that contributes to scattering at nonzero impact parameter. Amplitudes in the $s$ and $u$ channels need not grow like $s^{l}$. In the example we have seen so far the growth in these channels is slower than $s^{l}$; below we will also encounter examples in which the growth is faster. 


\section{L.1.1 Angular dependence and spherical harmonics}

In this subsubsection we will rewrite the first of the three terms (L.8) in the center of mass frame, i.e. the frame in which $p_{1}+p_{2}$ is a vector that points in the time direction. In this frame the $\Theta$ projects onto space. $p_{1}-p_{2}$ and $p_{3}-p_{4}$ are purely spatial vectors. In this frame $\left|\vec{p}_{1}\right|=\left|\vec{p}_{2}\right|=p$ and

$$
s=4 p^{2}, \quad t=2 p^{2}(1-\cos \theta), \quad u=2 p^{2}(1+\cos \theta), \quad \text { so } \frac{u-t}{s}=\cos \theta
$$

where $\theta$ is the scattering angle.

For the purposes of evaluating (L.1), $\Theta_{\mu \nu} \rightarrow \delta_{i j}$ ( $i$ and $j$ are spatial indices). In this frame the projector listed in (L.2) is a traceless symmetric tensor in the indices $\mu_{i}$ for any fixed values of the indices $\nu_{j}$. It follows that the projector is a spin $l$ scalar spherical harmonic of $\mathrm{SO}(D-1)$. The precise spherical harmonic obtained depends on the value of the indices $\nu_{i}$. In particular, if we dot all $\nu$ indices of the projector with a fixed vector $\vec{\beta}$ we obtain the unique spherical harmonic invariant under all the $\mathrm{SO}(D-2)$ rotations that keep $\vec{\beta}$ fixed. ${ }^{134}$ If we now dot the $\mu_{i}$ indices with a second vector $\vec{\alpha}$ such that the angle between $\vec{\beta}$ and $\vec{\alpha}$ is $\theta$, we obtain $|\alpha|^{l}|\beta|^{l}$ times the value of this special spherical harmonic at angle $\theta$. Plugging in the explicit formula for the projector yields the following explicit formula for the 'rotationally symmetric spherical harmonic' as a function of $\cos \theta$

$$
A_{l}^{D}(\cos \theta)=\sum_{p=0}^{[l / 2]} \frac{(-1)^{p} l !(2 l+D-2 p-5) ! !}{2^{p} p !(l-2 p) !(2 l+D-5) ! !}(\cos (\theta))^{l-2 p}
$$

Using the last of (L.9) we see that the first term in (L.8) is proportional to

$$
s^{l} \frac{A_{l}(\cos \theta)}{s-m^{2}} .
$$

where $\theta$ is the scattering angle [49].

\section{L.2 Four photon scattering from massive spin $s$ exchange}

The three particle photon-photon- $\mathcal{P}$ S-matrix is, in general, nonzero when $\mathcal{P}$ belongs to a larger class of representations than the traceless symmetric tensors. Although it is not difficult to enumerate the representations $\mathcal{P}$ which can consistently couple to two photons, we will not pause to record the results of this exercise, postponing it (and several other aspects of a systematic enumeration of exchange contributions to four photon scattering) to future work. In this subsection we simply focus on the special case for which $\mathcal{P}$ is traceless symmetric. ${ }^{135}$ Without loss of information we can choose the polarization of the exchanged particle to take the form

$$
e_{\mu_{1} \ldots \mu_{l}}=\left(\epsilon_{3}\right)_{\mu_{1}}\left(\epsilon_{3}\right)_{\mu_{2}} \ldots\left(\epsilon_{3}\right)_{\mu_{l}}, \quad\left(\epsilon_{3}\right)_{\mu} k_{3}^{\mu}=0, \quad \epsilon_{3} . \epsilon_{3}=0
$$

\footnotetext{
${ }^{134}$ If we choose the fixed vector to be the ' $\mathrm{z}$ ' axis we find the generalization of the $m=0$ spherical harmonic of $\mathrm{SO}(3)$.

${ }^{135}$ As the $D=4$ little group is $\mathrm{SO}(3)$, traceless symmetric tensors are the only vectorial representations that exist in this case, so the limited analysis presented in this subsection is actually exhaustive for the case $D=4$.
} 
We will now construct the most general 3 point coupling between two photons and a spin $l$ particle. Let the two photons have polarizations and momenta $\epsilon_{1}, k_{1}$ and $\epsilon_{2}, k_{2}$. Let the spin $l$ particle have mass $m$ and momentum $k_{3}$. The photon-photon-spin $l$ on-shell 3 point functions are necessarily polynomials of the following Lorentz invariant building blocks

$$
\begin{array}{lll}
A_{1}=\epsilon_{1} \cdot\left(k_{2}-k_{3}\right), & A_{2}=\epsilon_{2} \cdot\left(k_{1}-k_{3}\right), & A_{3}=\epsilon_{3} \cdot\left(k_{1}-k_{2}\right), \\
b_{12}=\epsilon_{1} \cdot \epsilon_{2}, & b_{23}=\epsilon_{2} \cdot \epsilon_{3}, & b_{13}=\epsilon_{1} \cdot \epsilon_{3},
\end{array}
$$

The most general polynomial of degree 1 in $\epsilon_{1}$ and $\epsilon_{2}$ and of degree $l$ in $\epsilon_{3}$ is a linear combination of the five structures

$$
A_{1} A_{2} A_{3}^{l}, \quad b_{12} A_{3}^{l} \quad b_{13} A_{2} A_{3}^{l-1} \quad b_{23} A_{1} A_{3}^{l-1} \quad b_{13} b_{23} A_{3}^{l-2}
$$

Under the interchange

$$
\begin{aligned}
\left(\epsilon_{1}, k_{1}\right) & \leftrightarrow\left(\epsilon_{2}, k_{2}\right) \\
A_{1} & \leftrightarrow A_{2}, b_{12} \leftrightarrow b_{12}, b_{13} \leftrightarrow b_{23}, A_{3} \leftrightarrow-A_{3}
\end{aligned}
$$

When $l$ is odd, the only Bose symmetric linear combination of the five structures listed in (L.11) is proportional to

$$
\left(A_{2} b_{13}+A_{1} b_{23}\right) A_{3}^{l-1}
$$

When $l$ is even, on the other hand, any linear combination of the following four structures

$$
A_{1} A_{2} A_{3}^{l}, \quad b_{12} A_{3}^{l} \quad b_{13} A_{2} A_{3}^{l-1}-b_{23} A_{1} A_{3}^{l-1} \quad b_{13} b_{23} A_{3}^{l-2}
$$

is Bose symmetric.

We now turn to the constraints imposed by the photonic gauge invariance. Under gauge transformation $\epsilon_{i} \rightarrow \epsilon_{i}+c_{i} k_{i}$ the building blocks transform as

$$
\begin{aligned}
& \delta A_{1}=-c_{1} m^{2}, \quad \delta A_{2}=-c_{2} m^{2}, \quad \delta A_{3}=0, \\
& \delta b_{12}=\frac{1}{2} c_{1} A_{2}+\frac{1}{2} c_{2} A_{1}, \quad \delta b_{23}=-\frac{1}{2} c_{2} A_{3}, \quad \delta b_{13}=\frac{1}{2} c_{1} A_{3}
\end{aligned}
$$

It is easily verified that the odd structure (L.14) is not gauge invariant. As a consequence the three point functions between two photons and a traceless symmetric $l$ rank tensor vanishes when $l$ is odd.

When $l$ is even, on the other hand, it is easily verified that the most general Bose symmetric gauge invariant 3 point function is a linear combination of the two structures $C_{1}$ and $C_{2}$ where

$$
\begin{aligned}
& C_{1}=A_{3}^{l}\left(A_{1} A_{2}+2 m^{2} b_{12}\right) \\
& C_{2}=A_{3}^{l-2}\left(-b_{12} A_{3}^{2}+\left(b_{13} A_{2}-b_{23} A_{1}\right) A_{3}+2 m^{2} b_{13} b_{23}\right)
\end{aligned}
$$

$C_{1}$ is proportional to the S-matrix that follows from the Lagrangian

$$
\left(\partial_{\mu_{1}} \ldots \partial_{\mu_{l}} F_{\alpha \beta}\right) F^{\alpha \beta} S_{\mu_{1} \ldots \mu_{l}}
$$


while $C_{2}$ is proportional to the S-matrix that follows from the Lagrangian

$$
\left(\partial_{\mu_{3}} \ldots \partial_{\mu_{l}} F_{\mu_{1} \alpha}\right) F_{\mu_{2} \alpha} S_{\mu_{1} \ldots \mu_{l}}
$$

where $S_{\mu_{1} \ldots \mu_{l}}$ represents the linearized spin $l$ field.

The four point function that follows by stitching two $C_{1}$ exchanges through the spin $l$ propagator is particularly simple. ${ }^{136}$ It is proportional to $E_{\mathbf{3}, 1}$. The S-matrix is (5.20) with the function $\mathcal{F}^{E_{\mathbf{3}, 2}}(t, u)=0, \mathcal{F}^{E_{\mathbf{S}}}(t, u)=0$ but

$$
\mathcal{F}^{E_{3,1}}(t, u)=\frac{c s^{l}}{m^{2}-s} A_{l}^{D}\left(\frac{u-t}{s}\right)
$$

where $c$ is a real number.

Regge growth. Unlike the scalar case the analysis here is a bit different because of the momenta dependence of the tensor structure $E_{\mathbf{3}, 1}$ multiplying $\mathcal{F}^{E_{\mathbf{3}, 1}}$. The Regge limit i.e. large $s$ at fixed $t$ limit of the S-matrix yields the following behavior for each channel.

$$
\begin{aligned}
\mathcal{S}_{s} & \rightarrow s^{l-1}\left(s^{2} e_{\mathbf{S}}-s^{2} e_{\mathbf{3}, 2}^{(1)}+s^{2} e_{\mathbf{3}, 1}^{(1)}\right) \\
\mathcal{S}_{t} & \rightarrow \frac{s^{l}}{m^{2}-t}\left(t^{2} e_{\mathbf{S}}-t^{2} e_{\mathbf{3}, 2}^{(2)}+t^{2} e_{\mathbf{3}, 1}^{(2)}\right) \\
\mathcal{S}_{u} & \rightarrow-s^{l-1}\left(s^{2} e_{\mathbf{S}}-s^{2} e_{\mathbf{3}, 2}^{(3)}+s^{2} e_{\mathbf{3}, 1}^{(3)}\right) \\
\mathcal{S}_{s}+\mathcal{S}_{u} & \rightarrow s^{l+1}\left(\left(e_{\mathbf{3}, 2}^{(3)}-e_{\mathbf{3}, 2}^{(1)}\right)+\left(e_{\mathbf{3}, 1}^{(1)}-e_{\mathbf{3}, 1}^{(3)}\right)\right) .
\end{aligned}
$$

As in the case of 4 scalar scattering, the $t$ channel exchange gives rise to a term that scales like $s^{l}$; the coefficient of this term is non analytic in $t$. It follows that the exchange contribution for $l>2$ grows faster than $s^{2}$ (in a way that cannot be cancelled by a local counter-term). Of course the $t$ channel exchange contributions from $l=2$ and $l=0$ grow no faster than $s^{2}$.

Let us now study $s$ and $u$ channel exchanges. Unlike the case of scalars, these channels yield the dominant contribution $-\propto s^{l+1}-$ to photon scattering. As in the case of scalar scattering subsection L.1, the contributions from $s$ and $u$ exchange appear with opposite signs. In this case, however, the two contributions appear with different polarization dependences, and hence do not cancel for generic choice of the polarization vectors. As for the scalars, however, this leading behavior can be cancelled by a contact term proportional to

$$
\operatorname{Tr}\left(\partial_{\mu_{1}} \ldots \partial_{\mu_{l-1}} F \partial_{\mu_{1}} \ldots \partial_{\mu_{l-1}} F\right) \operatorname{Tr}\left(F^{2}\right)
$$

provided that $l \geq 2$. It follows that the combined contribution of spin $l$ exchange plus the canceling counter-term grows no faster than $s^{2}$ for $l=2$ (the same is true for $l=0$; in this case there is no canceling counter-term).

\footnotetext{
${ }^{136}$ The most general exchange contribution of a massive spin $l$ particle is given by stitching together two three point functions of the form $a C_{1}+b C_{2}$. We leave the analysis of this general case to future work.
} 


\section{L.3 Massive spin $l$ exchange contribution to 4 graviton scattering}

The scalar Lagrangian (7.19) and the Spin 2 Lagrangian (7.33) are the first two members of a one parameter Lagrangians that describe the three point coupling between massive spin $l$ particles - i.e. massive particles transforming in the traceless symmetric $l$ index representation of $\mathrm{SO}(D-1)$ with even $l$ - with two gravitons. The Lagrangians in question are

$$
\nabla_{\mu_{1}} \nabla_{\mu_{2}} \ldots \ldots \nabla_{\mu_{l}} R_{a b c d} R^{a b c d} S^{\mu_{1} \mu_{2} \ldots \ldots \mu_{l}}
$$

In this subsubsection we compute the contribution of exchange amplitudes sewing two of these three point functions to 4 graviton scattering. ${ }^{137}$ The resultant S-matrix clearly has the same index structure as the Lagrangian $\left(R_{a b c d} R^{a b c d}\right)^{2}$. As a consequence, when the Smatrix is written in the form K.1, the only nonzero function is $\mathcal{F}^{G_{3,1}}(t, u)$. Explicitly we find

$$
\mathcal{F}^{G_{3,1}}(t, u)=\frac{s^{l}}{m^{2}-s} A_{l}^{D}\left(\frac{u-t}{s}\right)
$$

Regge growth. Here we analyze the Regge behavior from sewing the three point functions arising from (L.20). We list the behavior channel by channel:

$$
\begin{aligned}
& \mathcal{S}_{s} \rightarrow s^{l-1}\left(s^{4}\left(-1+\epsilon_{1}^{\perp} \cdot \epsilon_{2}^{\perp}\right)^{2}\left(-1+\epsilon_{3}^{\perp} \cdot \epsilon_{4}^{\perp}\right)^{2}\right) \\
& \mathcal{S}_{t} \rightarrow s^{l}\left(t^{4}\left(-1+\epsilon_{1}^{\perp} \cdot \epsilon_{3}^{\perp}\right)^{2}\left(-1+\epsilon_{2}^{\perp} \cdot \epsilon_{4}^{\perp}\right)^{2}\right) \\
& \mathcal{S}_{u} \rightarrow-s^{l-1}\left(s^{4}\left(-1+\epsilon_{1}^{\perp} \cdot \epsilon_{4}^{\perp}\right)^{2}\left(-1+\epsilon_{2}^{\perp} \cdot \epsilon_{3}^{\perp}\right)^{2}\right)
\end{aligned}
$$

As expected, the contribution from the $t$ channel scales like $s^{l}$. For $l \geq 4$ this manifestly non-local contribution grows faster than $s^{2}$. The special cases $l=0$ and $l=2$ have already been analyzed in detail above; recall that we found that in these cases the combined contribution of $s$ and $u$ channel exchange grows faster than $s^{2}$ even after any possible local counter-term subtraction.

Open Access. This article is distributed under the terms of the Creative Commons Attribution License (CC-BY 4.0), which permits any use, distribution and reproduction in any medium, provided the original author(s) and source are credited.

\section{References}

[1] S. Minwalla, Two questions about gravity, talk at Strings 2018, https://indico.oist.jp/indico/event/5/picture/141.pdf, (2018).

[2] X.O. Camanho, J.D. Edelstein, J. Maldacena and A. Zhiboedov, Causality constraints on corrections to the graviton three-point coupling, JHEP 02 (2016) 020 [arXiv:1407.5597] [INSPIRE].

\footnotetext{
${ }^{137}$ Two gravitons cannot couple to spin $l$ particles when $l$ is odd. When $l$ is even there are 3 independent coupling structures. In this subsubsection we focus on only one of these three structures as an example. We hope to return to the general case in future work.
} 
[3] M. Kulaxizi, A. Parnachev and A. Zhiboedov, Bulk phase shift, CFT Regge limit and Einstein gravity, JHEP 06 (2018) 121 [arXiv: 1705.02934] [INSPIRE].

[4] A. Adams, N. Arkani-Hamed, S. Dubovsky, A. Nicolis and R. Rattazzi, Causality, analyticity and an IR obstruction to UV completion, JHEP 10 (2006) 014 [hep-th/0602178] [INSPIRE].

[5] B. Bellazzini, C. Cheung and G.N. Remmen, Quantum gravity constraints from unitarity and analyticity, Phys. Rev. D 93 (2016) 064076 [arXiv:1509.00851] [InSPIRE].

[6] N. Afkhami-Jeddi, T. Hartman, S. Kundu and A. Tajdini, Einstein gravity 3-point functions from conformal field theory, JHEP 12 (2017) 049 [arXiv:1610.09378] [INSPIRE].

[7] N. Afkhami-Jeddi, T. Hartman, S. Kundu and A. Tajdini, Shockwaves from the operator product expansion, JHEP 03 (2019) 201 [arXiv: 1709.03597] [INSPIRE].

[8] M. Kologlu, P. Kravchuk, D. Simmons-Duffin and A. Zhiboedov, Shocks, superconvergence and a stringy equivalence principle, arXiv:1904.05905 [INSPIRE].

[9] N. Arkani-Hamed, T.-C. Huang and Y.-T. Huang, Scattering amplitudes for all masses and spins, arXiv:1709.04891 [INSPIRE].

[10] B. Henning, X. Lu, T. Melia and H. Murayama, Hilbert series and operator bases with derivatives in effective field theories, Commun. Math. Phys. 347 (2016) 363 [arXiv: 1507.07240] [INSPIRE].

[11] B. Henning, X. Lu, T. Melia and H. Murayama, Operator bases, $S$-matrices and their partition functions, JHEP 10 (2017) 199 [arXiv:1706.08520] [INSPIRE].

[12] J. Maldacena, S.H. Shenker and D. Stanford, A bound on chaos, JHEP 08 (2016) 106 [arXiv: 1503.01409] [INSPIRE].

[13] S. Caron-Huot, Analyticity in spin in conformal theories, JHEP 09 (2017) 078 [arXiv: 1703.00278] [INSPIRE].

[14] D. Simmons-Duffin, D. Stanford and E. Witten, A spacetime derivation of the Lorentzian OPE inversion formula, JHEP 07 (2018) 085 [arXiv:1711.03816] [INSPIRE].

[15] P. Kravchuk and D. Simmons-Duffin, Light-ray operators in conformal field theory, JHEP 11 (2018) 102 [arXiv : 1805.00098] [INSPIRE].

[16] I. Halder, Global symmetry and maximal chaos, arXiv:1908.05281 [INSPIRE].

[17] M. Mezei and G. Sárosi, Chaos in the butterfly cone, JHEP 01 (2020) 186 [arXiv: 1908.03574] [INSPIRE].

[18] R.R. Poojary, BTZ dynamics and chaos, arXiv:1812.10073 [INSPIRE].

[19] V. Jahnke, K.-Y. Kim and J. Yoon, On the chaos bound in rotating black holes, JHEP 05 (2019) 037 [arXiv: 1903.09086] [INSPIRE].

[20] L. Cornalba, Eikonal methods in AdS/CFT: Regge theory and multi-reggeon exchange, arXiv:0710.5480 [INSPIRE].

[21] I. Heemskerk, J. Penedones, J. Polchinski and J. Sully, Holography from conformal field theory, JHEP 10 (2009) 079 [arXiv:0907.0151] [INSPIRE].

[22] S.H. Shenker and D. Stanford, Stringy effects in scrambling, JHEP 05 (2015) 132 [arXiv: 1412.6087] [INSPIRE].

[23] G.J. Turiaci and A. Zhiboedov, Veneziano amplitude of Vasiliev theory, JHEP 10 (2018) 034 [arXiv:1802.04390] [INSPIRE]. 
[24] D. Meltzer and E. Perlmutter, Beyond $a=c$ : gravitational couplings to matter and the stress tensor OPE, JHEP 07 (2018) 157 [arXiv: 1712.04861] [INSPIRE].

[25] N. Afkhami-Jeddi, S. Kundu and A. Tajdini, A conformal collider for holographic CFTs, JHEP 10 (2018) 156 [arXiv:1805.07393] [INSPIRE].

[26] P. Kravchuk and D. Simmons-Duffin, Counting conformal correlators, JHEP 02 (2018) 096 [arXiv: 1612.08987] [INSPIRE].

[27] A. Dymarsky, On the four-point function of the stress-energy tensors in a CFT, JHEP 10 (2015) 075 [arXiv:1311.4546] [INSPIRE].

[28] M.S. Costa, J. Penedones, D. Poland and S. Rychkov, Spinning conformal blocks, JHEP 11 (2011) 154 [arXiv:1109.6321] [INSPIRE].

[29] R. de Mello Koch, P. Rabambi, R. Rabe and S. Ramgoolam, Counting and construction of holomorphic primary fields in free $\mathrm{CFT}_{4}$ from rings of functions on Calabi-Yau orbifolds, JHEP 08 (2017) 077 [arXiv: 1705.06702] [INSPIRE].

[30] R. de Mello Koch and S. Ramgoolam, Free field primaries in general dimensions: counting and construction with rings and modules, JHEP 08 (2018) 088 [arXiv:1806.01085] [INSPIRE].

[31] J.M. Maldacena and G.L. Pimentel, On graviton non-Gaussianities during inflation, JHEP 09 (2011) 045 [arXiv: 1104.2846] [INSPIRE].

[32] S. Giombi, S. Prakash and X. Yin, A note on CFT correlators in three dimensions, JHEP 07 (2013) 105 [arXiv: 1104.4317] [INSPIRE].

[33] M.S. Costa, J. Penedones, D. Poland and S. Rychkov, Spinning conformal correlators, JHEP 11 (2011) 071 [arXiv: 1107.3554] [INSPIRE].

[34] C. Lanczos, A remarkable property of the Riemann-Christoffel tensor in four dimensions, Annals Math. 39 (1938) 842 [INSPIRE].

[35] D. Lovelock, The Einstein tensor and its generalizations, J. Math. Phys. 12 (1971) 498 [INSPIRE].

[36] B. Sundborg, The Hagedorn transition, deconfinement and $N=4$ SYM theory, Nucl. Phys. B 573 (2000) 349 [hep-th/9908001] [INSPIRE].

[37] O. Aharony, J. Marsano, S. Minwalla, K. Papadodimas and M. Van Raamsdonk, The Hagedorn/deconfinement phase transition in weakly coupled large $N$ gauge theories, Adv. Theor. Math. Phys. 8 (2004) 603 [hep-th/0310285] [inSPIRE].

[38] J.H. Schwarz, Superstring theory, Phys. Rept. 89 (1982) 223 [INSPIRE].

[39] S. Deser and D. Seminara, Tree amplitudes and two loop counterterms in D $=11$ supergravity, Phys. Rev. D 62 (2000) 084010 [hep-th/0002241] [INSPIRE].

[40] S.A. Fulling, R.C. King, B.G. Wybourne and C.J. Cummins, Normal forms for tensor polynomials. 1: the Riemann tensor, Class. Quant. Grav. 9 (1992) 1151 [INSPIRE].

[41] S.D. Chowdhury and J.R. David, Global gravitational anomalies and transport, JHEP 12 (2016) 116 [arXiv: 1604.05003] [INSPIRE].

[42] S. Sannan, Gravity as the limit of the type II superstring theory, Phys. Rev. D 34 (1986) 1749 [INSPIRE]. 
[43] G.J. Turiaci, An inelastic bound on chaos, JHEP 07 (2019) 099 [arXiv:1901.04360] [INSPIRE].

[44] S.B. Giddings, S. Kachru and J. Polchinski, Hierarchies from fluxes in string compactifications, Phys. Rev. D 66 (2002) 106006 [hep-th/0105097] [INSPIRE].

[45] G. Veneziano, S. Yankielowicz and E. Onofri, A model for pion-pion scattering in large- $N$ QCD, JHEP 04 (2017) 151 [arXiv: 1701.06315] [INSPIRE].

[46] L.P.S. Singh and C.R. Hagen, Lagrangian formulation for arbitrary spin 1. The boson case, Phys. Rev. D 9 (1974) 898 [inSPIRE].

[47] R.L. Ingraham, Covariant propagators and vertices for higher spin bosons, Prog. Theor. Phys. 51 (1974) 249 [INSPIRE].

[48] P. Nayak, R.R. Poojary and R.M. Soni, A note on S-matrix bootstrap for amplitudes with linear spectrum, arXiv:1707.08135 [INSPIRE].

[49] X. Bekaert, E. Joung and J. Mourad, On higher spin interactions with matter, JHEP 05 (2009) 126 [arXiv:0903.3338] [INSPIRE]. 